\title{
Sobre os P-subespaços em uma train álgebra de posto 3
}

Roseli Arbach Fernandes de Oliveira

Tese apresentada

ao

Instituto de Matemática e Estatística

da

Universidade de São Paulo

para a obtenção do grau de Doutor

em

Matemática

Área de concentração: Álgebra

Orientador: Prof. Dr. Roberto Celso Fabrício Costa

- São Paulo, outubro de 1997 - 


\section{Sobre os P-subespaços em uma train álgebra de posto 3}

Este exemplar corresponde à redação final da dissertação devidamente corrigida e defendida por Roseli Arbach Fernandes de Oliveira e aprovada pela comissão julgadora.

São Paulo. 15 de dezembro 1997.

Banca examinadora:

- Prof. Dr. Roberto Celso Fabrício Costa (Orientador) - IME - USP

- Prof. Dr. Luiz Antônio Peresi - IME - USP

- Prof. Dr. Juan Carlos Gutiérrez Fernandéz - Universidade de Oviedo

- Prof. Dr. Antônio José Engler - IMECC - UNICAMP.

- Profa. Dra. Ires Dias - ICMSC - USP 
"Nâo vemos o que vemos

Vemos o que somos"

Fernando Pessoa

Para

Meu marido Luis. com quem divido há 16 anos todos os momentos de tristeza e de alegria. Meus filhos. Fábio e Ricardo, cujas existências m.e dâo força para seguir adiante. D. Rosa. mâe e amiga, presente em todos os momentos de minha vida. Meu pai Abdo (in memorian). exemplo de trabalho e integridade. 


\section{Agradecimentos}

Embrenhar-se numa jornada como esta exige muito sacrifício. São muitas horas de dedicação diária, por um longo período; são muitas horas de sono trocadas por muitas horas de estudo e dessa troca, diversas vezes, surgem poucos resultados. Mas tudo isso é sacrifício pessoal, do qual não podemos (ou não devemos) nos queixar, pois ele é consequência de uma opção também pessoal. Refiro-me ao sacrifício que é imposto à nossa família, aos nossos filhos, pois durante todo tempo em que estive envolvida com o doutorado, embora a escolha tivesse sido minha, o envolvimento foi de todos. Por isso agradeço à minha mãe, D. Rosa, que tomou a si a responsabilidade de cuidar de meus filhos; agradeço a meu marido, Luis, que sempre esteve ao meu lado e me incentivou nas horas em que batia um grande desânimo; aos meus filhos, Fábio e Ricardo. que desde muito pequenos tiveram que aprender a conviver com a ausência dos pais, em benefício de nosso trabalho. Durante esse período pude também contar com a ajuda de meu irmão Nelson, de minha cunhada Daisy, e de minha tia Mentaha; a todos eles deixo meus agradecimentos. Agradeço também aos meus colegas do Departamento de Matemática da FEIS-UNESP, em particular ao Prof. Aldir Chaves Brasil Júnior e Prof. Luis Antônio Fernandes de Oliveira, que sempre se dispuseram a me ouvir. À CAPES meus agradecimentos pelo financiamento parcial, em forma de bolsa de estudos, deste trabalho.

Não posso deixar de citar algumas pessoas que conheci no IME-USP, em especial Lúcia Satie Ikemoto, Juaci Picanço da Silva, Prof. Henrique Guzzo Jr. e Maria Luiza Paiva e Silva Lelis. A convivência com eles no período em que estive em São Paulo, especialmente nos últimos cinco 
meses, tornou mais agradável esta fase difícil em que tive que ficar longe de minha família e por isso registro aqui minha gratidão a todos eles.

Quando pensei em estudar Álgebra, foi-me sugerido procurar pelo Prof. Roberto Costa. Foi um sábio conselho que recebi e por ele agradeço sinceramente ao meu colega de Departamento e amigo Prof. João Toledo, que além da valiosa sugestão, prontificou-se a me apresentar ao Prof. Roberto, em uma de suas visitas a Ilha Solteira.

Eu me considero uma pessoa de sorte e uma das grandes sortes que tive foi ter sido aceita como aluna pelo Prof. Roberto Costa. Ser um excelente Pesquisador e Matemático não é uma tarefa fácil. Ser um excelente Pesquisador e Matemático, além disso, conservar a humildade e ainda manter-se um ser humano íntegro é bem mais difícil. Tudo isso faz com que eu admire profundamente o Prof. Roberto Costa. Há pessoas a quem dizer apenas "muito obrigado" não traduz o quanto lhes somos gratos, o quanto lhes devemos, mas mesmo assim isso é o único que sabemos dizer. Ao Prof. Roberto Costa, meu muito obrigada. 


\section{Abstract}

In this work, we will introduce the concepts of $\mathrm{P}$-monomials and $\mathrm{P}$ subspaces in a train algebra $\mathrm{A}$ of rank 3 . We study in details those of degree $\leq 4$ and we will find some algebraic and numerical invariants of A. Moreover, under some determined conditions, we obtain the minimal dimension of $\mathrm{A}$ and it is found minimal examples. Finally, we will introduce and study the concept of relative invariance.

\section{Resumo}

Neste trabalho, introduzimos os conceitos de P-monômios e P-subespaços em uma train álgebra $\mathrm{A}$ de posto 3 . Estudamos detalhadamente aqueles de grau $\leq 4$ e encontramos alguns invariantes algébricos e numéricos de A. Além disso, sob determinadas condições, obtemos a dimensão mínima de A e são encontrados exemplos minimais. Finalmente, introduzimos e estudamos o conceito de invariância relativa. 


\section{Conteúdo}

$1 \quad P$-subespaços em train álgebras $\quad 1$

1.1 Breve histórico . . . . . . . . . . . . . . . . . . . 1

1.2 Resultados básicos . . . . . . . . . . . . . . . . . . . 2

1.3 Os subespaços $\mathrm{p}(\mathrm{U}, \mathrm{V})$ : problemas .......... 7

2 Sobre os subespaços $\mathrm{U}^{n} \oplus \mathrm{U}^{n+1} \quad \mathbf{1 5}$

2.1 Invariância da dimensão de $\mathrm{U}^{n} \ldots \ldots \ldots \ldots$

3 Soluções parciais do Problema 2 21

3.1 Alguns casos do Problema 2 . . . . . . . . . . . 21

4 Sobre a dimensão de A, baseada em P-monômios 27

4.1 Lemas auxiliares . . . . . . . . . . . . . . . . . . 27

4.2 Resultados e exemplos . . . . . . . . . . . . . 33

5 Sobre a invariância dos P-subespaços $\quad 54$

5.1 Os P-subespaços de grau $\leq 4 \ldots \ldots \ldots \ldots$

5.2 Proposições auxiliares: geradores de $\mathrm{m}(\mathrm{U} . \mathrm{V}) \ldots \ldots . . \quad 55$

5.3 Invariância: Grupos I e II . . . . . . . . . . . . . . . . . . 63

5.4 Sobre o Grupo II . . . . . . . . . . . . . . . 81

6 Ideais de tipo polinomial 94

6.1 O estudo do Grupo I . . . . . . . . . . . . . . . . . . . . 94

6.2 Ideais gerados pelos monômios de Peirce . . . . . . . . . . . 98

6.3 Propriedades de ideais . . . . . . . . . . . . . 102 
7 Sobre a invariância relativa dos P-subespaços 110 7.1 Invariância relativa: definição . . . . . . . . . . . . . 110

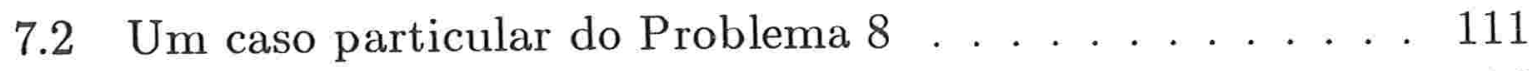

7.3 Problema 9: casos particulares . . . . . . . . . 116

8 Apêndice $\quad 129$

9 Bibliografia 133 


\section{Capítulo 1}

\section{$\mathbf{P}$-subespaços em train álgebras}

\subsection{Breve histórico}

No período de 1939 a 1951, I. M. H. Etherington escreveu uma série de artigos nos quais introduziu vários conceitos novos em álgebra não associativa, na tentativa de construir modelos para o estudo de Genética de Populações, ver [11] a [15]. Em particular, ele considerou as t-álgebras, que são álgebras não associativas, munidas de um homomorfismo não nulo no corpo de escalares, satisfazendo uma equação polinomial, que envolve também este homomorfismo. O estudo destas álgebras esteve esquecido durante muitos anos porém tem sido mais intenso nos últimos 10 anos. Agora já se conhecem os resultados fundamentais e pode-se avaliar a dificuldade para tentar uma classificação destas álgebras. As referências colocadas ao final dão uma idéia dos problemas que estão sendo estudados nesta área. Em especial, os artigos [9], [20]. [21] contêm resultados recentes e significativos sobre o tema das t-álgebras. O artigo [28] contém uma análise crítica dos conceitos básicos da teoria das álgebras que são relevantes em Genética de Populações. Em especial, contém o material clássico sobre as t-álgebras, bem como a motivação para a sua introdução por Etherington. Nesta tese, fazemos um estudo bastante detalhado de algumas propriedades de subespaços de tipo polinomial que são originários de uma decomposição de Peirce de uma t-álgebra de posto 3 e têm grau não superior a 4 (ver mais detalhes no parágrafo 3 deste Capítulo). Esperamos que o conhecimento destas propriedades possa ser 
utilizado quando se pensar em fazer uma classificação destas álgebras.

\subsection{Resultados básicos}

Nesta seção, introduziremos, sem demonstrações, os conceitos e resultados básicos já conhecidos sobre train álgebras de posto 3 e que são necessários para a leitura deste trabalho.

Sejam F um corpo de característica diferente de 2 e A uma F-álgebra de dimensão finita, comutativa, não necessariamente associativa. Se existir um homomorfismo de álgebras, não nulo, $\omega: \mathrm{A} \rightarrow \mathrm{F}$ então o par $(\mathrm{A}, \omega)$ será chamado uma álgebra bárica (ou álgebra ponderada) e $\omega$ a sua função peso. (Em geral, o número de homomorfismos não nulos $\omega: \mathrm{A} \rightarrow \mathrm{F}$ pode variar entre 0 e $\operatorname{dim} \mathrm{A}$. Álgebras nilpotentes não possuem nenhum homomorfismo não nulo $\omega$ como acima; no outro extremo, $\mathrm{A}=\mathrm{F}^{n}$ possui $n$ homomorfismos não nulos com valores em $\mathrm{F}$, a saber as $n$ projeções de $\mathrm{F}^{n}$ em F). Se $a$ é um elemento arbitrário de A, o escalar $\omega(\mathrm{a})$ é chamado o peso de a. $\mathrm{O}$ núcleo $\mathrm{N}$ da função peso é um ideal de codimensão $1 \mathrm{em}$ A. Os ideais de A, contidos em $\mathrm{N}$, são ditos ideais báricos de A. Se $I$ é um ideal bárico de A, então A/I é também bárica, sendo a função peso $\bar{\omega}: \mathrm{A} / \mathrm{I} \rightarrow \mathrm{F}$ definida por $\bar{\omega}(\mathrm{a}+\mathrm{I})=\omega(\mathrm{a}), \mathrm{a} \in \mathrm{A}$. Se $(\mathrm{A}, \omega)$ é uma álgebra bárica e $a$ é um elemento de $\mathrm{A}$ tal que $\omega(\mathrm{a})=1$, então $\mathrm{A}=\mathrm{Fa} \oplus \mathrm{N}$ pois. para todo $\mathrm{x}$ de $\mathrm{A}$, tem-se $\mathrm{x}=\omega(\mathrm{x}) \mathrm{a}+[\mathrm{x}-\omega(\mathrm{x}) \mathrm{a}]$ e $\mathrm{x}-\omega(\mathrm{x})$ a tem peso 0 .

$\mathrm{Na}$ teoria das álgebras não associativas há várias maneiras de se definir potências de elementos. Usaremos neste trabalho apenas as potências principais, definidas recursivamente por $\mathrm{a}^{1}=\mathrm{a}, \mathrm{a}^{n+1}=\mathrm{a}^{n} \mathrm{a}$, para todo $\mathrm{n} \geq 1$. Observemos que, em geral, $\mathrm{a}^{i} \mathrm{a}^{j} \neq \mathrm{a}^{i+j}$. Quando $\mathrm{a}^{i} \mathrm{a}^{j}=\mathrm{a}^{i+1}$ para todo $a \in A, i, j \geq 1$, dizemos que $A$ é de potências associativas. As grandes classes de álgebras não associativas ( Lie, Jordan,alternativas,...) são de potências associativas. As train álgebras não são, em geral. de potências associativas.

Para cada álgebra não associativa $B$, definimos $\mathrm{B}^{1}=\mathrm{B}, \mathrm{B}^{2}=\mathrm{BB}$, $\mathrm{B}^{3}=\mathrm{B}^{2} \mathrm{~B}^{1}+\mathrm{B}^{1} \mathrm{~B}^{2}=(\mathrm{BB}) \mathrm{B}+\mathrm{B}(\mathrm{BB})$ e de modo geral $\mathrm{B}^{i}=\mathrm{B}^{1} \mathrm{~B}^{i-1}+$ $+\mathrm{B}^{2} \mathrm{~B}^{i-2}+\ldots+\mathrm{B}^{i-2} \mathrm{~B}^{2}+\mathrm{B}^{i-1} \mathrm{~B}$. Dizemos que $B$ é nilpotente se existe 
$k$ tal que $\mathrm{B}^{k}=0$. É fácil ver que a álgebra $B$ é nilpotente se e somente se existir um inteiro positivo $k$ t tal que o produto de quaisquer $k$ elementos de $B$ é nulo, não importando a forma como os elementos estão associados. O menor inteiro $k$ para o qual esta propriedade é satisfeita é chamado o índice de nilpotência de $B$.

Para cada álgebra não associativa $B$, definimos $\mathrm{B}^{(0)}=\mathrm{B}, \mathrm{B}^{(1)}=\mathrm{BB}$, $\ldots, \mathrm{B}^{(i)}=\mathrm{B}^{(i-1)} \mathrm{B}^{(i-1)}$. Então $B$ é solúvel se existe um inteiro $k$ tal que $\mathrm{B}^{(k)}=0$. O menor inteiro $k$ tal que $\mathrm{B}^{(k)}=0$ é chamado o índice de solubilidade de $B$. É fácil ver que se $B$ é nilpotente então $B$ é solúvel, mas a recíproca é falsa em geral. No caso de álgebras associativas, os conceitos de nilpotência e solubilidade coincidem.

Uma álgebra bárica $(\mathrm{A}, \omega)$ sobre o corpo $\mathrm{F}$ é dita uma train álgebra (ou, simplesmente t-álgebra) se existirem escalares $\gamma_{1}, \gamma_{2}, \ldots, \gamma_{n-1} \in \mathrm{F}$ tais que todo elemento $a$ de A satisfaz a equação $\mathrm{a}^{n}+\gamma_{1} \omega(\mathrm{a}) \mathrm{a}^{n-1}+\ldots+$ $+\gamma_{n-1} \omega(\mathrm{a})^{n-1} \mathrm{a}=0$. Nessas condições temos $1+\gamma_{1}+\gamma_{2}+\ldots+$ $+\gamma_{n-1}=0$. Para ver isso, basta calcularmos o peso do elemento $\mathrm{a}^{n}+\gamma_{1} \omega(\mathrm{a}) a^{n-1}+\ldots+\gamma_{n-1} \omega(\mathrm{a})^{n-1} \mathrm{a}$, sendo $a$ um elemento de A de peso diferente de zero.

O polinômio $\mathrm{f}(\mathrm{x})=\mathrm{x}^{n}+\gamma_{1} \omega(\mathrm{x}) \mathrm{x}^{n-1}+\ldots+\gamma_{n-1} \omega(\mathrm{x})^{n-1} \mathrm{x}$ é chamado train polinômio (ou t-polinômio) de grau $n$ e o grau do polinômio de menor grau satisfeito por A é chamado o posto de $A$. É fácil ver que este polinômio é único. Se A é uma t-álgebra de posto $n$, os elementos do núcleo de $\omega$ satisfazem a identidade $x^{n}=0$. Como consequência, segue-se que o homomorfismo peso de $\mathrm{A}$ é univocamente determinado. A demonstração deste fato pode ser encontrada em [36, pag. 36] ou em [28. Proposição 3.2]. Em nosso trabalho, estaremos interessados apenas em t-álgebras de posto 3 , isto é, álgebras báricas que satisfazem a equação

$$
\mathrm{x}^{3}-(1+\gamma) \omega(\mathrm{x}) \mathrm{x}^{2}+\gamma \omega(\mathrm{x})^{2} \mathrm{x}=0
$$

com $\gamma \in \mathrm{F}$. Resultados básicos sobre t-álgebras de posto 3 podem ser vistos em [6] e [36]. Em particular, todos os resultados enunciados nesta 
seção podem ser encontrados nestas referências. A teoria das t-álgebras de posto $\geq 4$ é muito mais complexa, veja por exemplo [16] e [18].

Assumiremos, em todo o trabalho, que $2 \gamma \neq 1$ e, como consequência, garantimos a existência em A de (pelo menos) um elemento idempotente de peso 1. De fato, é fácil ver que se $d \in A$ e $\omega(\mathrm{d})=1$ então $\mathbf{e}=(1-2 \gamma)^{-1}\left(d^{2}-2 \gamma d\right)$ é um idempotente de peso 1 . Relativamente a esse idempotente, temos a decomposição de Peirce de A na soma direta

$$
\mathrm{A}=\mathrm{Fe} \oplus \mathrm{U}_{\mathrm{e}} \oplus \mathrm{V}_{\mathrm{e}}
$$

sendo

$$
\begin{gathered}
\mathrm{U}_{\mathrm{e}}=\{\mathrm{u} \in \mathrm{N}: 2 \mathrm{eu}=\mathrm{u}\} \\
\mathrm{V}_{\mathrm{e}}=\{\mathrm{v} \in \mathrm{N}: \mathrm{ev}=\gamma \mathrm{v}\} \\
\mathrm{N}=\mathrm{U}_{\mathrm{e}} \oplus \mathrm{V}_{\mathrm{e}}
\end{gathered}
$$

Assim $\mathrm{U}_{e}$ e $\mathrm{V}_{e}$ são os subespaços próprios do operador linear $\mathrm{L}_{e}: \mathrm{N} \rightarrow \mathrm{N}$ dado por $\mathrm{n} \mapsto$ en, correspondentes aos seus valores próprios (distintos) $\frac{1}{2}$ e $\gamma$. Para cada $\mathrm{x} \in \mathrm{A}$ como em (1.2) temos $\mathrm{x}=\omega(\mathrm{x}) \mathrm{e}+\mathrm{u}+\mathrm{v}$, sendo $\mathrm{u} \in \mathrm{U}_{e} \mathrm{e} \mathrm{v} \in \mathrm{V}_{\epsilon}$. Esta expressão é a decomposição de Peirce do elennento $x$, relativamente a $e$. Quando $2 \gamma=1$, a equação (1.1) torna-se $2 \mathrm{x}^{3}+$ $-3 \omega(\mathrm{x}) \mathrm{x}^{2}+\omega(\mathrm{x})^{2} \mathrm{x}=0$ e a teoria torna-se mais difícil, pois não temos a decomposição (1.5) do núcleo de $\omega$.

As linearizações da equação (1.1), dadas por (1.6) e (1.7) abaixo. são também identidades em uma t-álgebra de posto 3 :

$$
\begin{gathered}
\mathrm{x}^{2} \mathrm{y}+2 \mathrm{x}(\mathrm{xy})-(1+\gamma)\left[2 \omega(\mathrm{x}) \mathrm{xy}+\omega(\mathrm{y}) \mathrm{x}^{2}\right]+\gamma\left[\omega(\mathrm{x})^{2} \mathrm{y}+2 \omega(\mathrm{xy}) \mathrm{x}\right]=0 \\
{[\mathrm{x}(\mathrm{yz})+\mathrm{y}(\mathrm{xz})+\mathrm{z}(\mathrm{xy})]-(1+\gamma)[\omega(\mathrm{x}) \mathrm{yz}+\omega(\mathrm{y}) \mathrm{xz}+\omega(z) \mathrm{xy}]+} \\
+\gamma[\omega(\mathrm{yz}) \mathrm{x}+\omega(\mathrm{xz}) \mathrm{y}+\omega(\mathrm{xy}) \mathrm{z}]=0
\end{gathered}
$$

Usando-se essas linearizações, provam-se as seguintes relações entre os subespaços $\mathrm{U}_{\epsilon}$ e $\mathrm{V}_{\epsilon}$ : 


$$
\begin{gathered}
\mathrm{U}_{\mathrm{e}}^{2} \subseteq \mathrm{V}_{\mathrm{e}} \\
\mathrm{U}_{\mathrm{e}} \mathrm{V}_{\mathrm{e}} \subseteq \mathrm{U}_{\mathrm{e}} \\
\mathrm{V}_{\mathrm{e}}^{2}=0 \\
\mathrm{U}_{\mathrm{e}}^{2 \mathrm{n}+1} \subseteq \mathrm{U}_{\mathrm{e}}(\mathrm{n} \geq 0) \\
\mathrm{U}_{\mathrm{e}}^{2 \mathrm{n}} \subseteq \mathrm{V}_{\mathrm{e}}(\mathrm{n} \geq 1)
\end{gathered}
$$

Para provar (1.8) tomamos $\mathrm{x}=\mathrm{e}, \mathrm{y}, \mathrm{z} \in \mathrm{U}_{e}$ em (1.7) e obtemos e(yz) + $+\mathrm{y}(\mathrm{ez})+\mathrm{z}(\mathrm{ey})-(1+\gamma) \mathrm{yz}=0$, ou seja $\mathrm{e}(\mathrm{yz})+\mathrm{yz}-\mathrm{yz}-\gamma \mathrm{yz}=0$ ou $\mathrm{e}(\mathrm{yz})=\gamma \mathrm{yz}$, o que significa que $\mathrm{yz} \in \mathrm{V}_{e}$ e assim $\mathrm{U}_{e}^{2} \subseteq \mathrm{V}_{e}$. As identidades (1.9) e (1.10) são provadas de maneira análoga. Para provar (1.11) e (1.12), usamos indução sobre $n$.

Usando-se (1.7), vemos que três elementos arbitrários $\mathrm{x}, \mathrm{y}, \mathrm{z}$ de peso 0 satisfazem a identidade de Jacobi, ou seja

$$
\mathrm{J}(\mathrm{x}, \mathrm{y}, \mathrm{z})=\mathrm{x}(\mathrm{yz})+\mathrm{y}(\mathrm{xz})+\mathrm{z}(\mathrm{xy})=0
$$

Esta identidade desempenha um papel fundamental neste trabalho. Por exemplo, através dela provamos (por indução sobre $i$ ) que para todo subespaço $X$ de $\mathrm{A}$, vale a inclusão $\mathrm{X}^{i} \mathrm{X}^{j} \subseteq \mathrm{X}^{i+i}$. De fato, se $\mathrm{i}=1$, para todo $j$ temos que $\mathrm{X}^{i} \mathrm{X}^{i}=\mathrm{XX}^{i}=\mathrm{X}^{i+1}=\mathrm{X}^{i+j}$. Suponhamos que $\mathrm{X}^{i-1} \mathrm{X}^{i} \subseteq$ $\subseteq \mathrm{X}^{i+j-1}$, para todo número natural $\mathrm{j} \geq 2$ e consideremos um gerador $y$ de $\mathrm{X}^{i} \mathrm{X}^{j}$. Então $\mathrm{y} \in \mathrm{X}^{i} \mathrm{X}^{j}=\left(\mathrm{XX}^{i-1}\right) \mathrm{X}^{j}$, isto é, $\mathrm{y}=\left(\mathrm{x}_{1} \mathrm{x}_{2}\right) \mathrm{x}$, sendo $\mathrm{x}_{1} \in$ $\in \mathrm{X}, \mathrm{x}_{2} \in \mathrm{X}^{i-1}$ e $\mathrm{x} \in \mathrm{X}^{j}$. De $\mathrm{J}\left(\mathrm{x}_{1}, \mathrm{x}_{2}, \mathrm{x}\right)=0$, obtemos $\mathrm{y}=-\mathrm{x}_{1}\left(\mathrm{x}_{2} \mathrm{x}\right)+$ $-\mathrm{x}_{2}\left(\mathrm{x}_{1} \mathrm{x}\right) \in \mathrm{X}\left(\mathrm{X}^{i-1} \mathrm{X}^{j}\right)+\mathrm{X}^{i-1}\left(\mathrm{XX}^{j}\right)$. Mas, da hipótese de indução, temos que $\mathrm{X}\left(\mathrm{X}^{i-1} \mathrm{X}^{j}\right) \subseteq \mathrm{XX}^{i+j-1}=\mathrm{X}^{i+j}$ e $\mathrm{X}^{i-1}\left(\mathrm{XX}^{j}\right)=\mathrm{X}^{i-1} \mathrm{X}^{j+1} \subseteq \mathrm{X}^{i+j}$. Logo, $\mathrm{y} \in \mathrm{X}^{i+j}$ e daí concluímos que $\mathrm{X}^{i} \mathrm{X}^{j} \subseteq \mathrm{X}^{i+j}$, para quaisquer números naturais $i$ e $j$.

Além disso, se $\mathrm{u}, \mathrm{u}_{1} \in \mathrm{U}_{\epsilon}, \mathrm{v} \in \mathrm{V}_{\epsilon}$ são elementos quaisquer desses subespaços, a partir de (1.13), provam-se as seguintes relações:

$$
\mathrm{u}(\mathrm{uv})=0
$$




$$
\begin{gathered}
(u v) v=0 \\
u^{2}(u v)=0 \\
(u v)^{2}=0 \\
u\left(u^{2} u_{1}\right)=0
\end{gathered}
$$

Em qualquer álgebra bárica $(\mathrm{A}, \omega)$ chama-se ideal de Etherington de $\mathrm{A}$ o ideal gerado pelos elementos $a^{2}-\omega(a) a$, com a $\in$ A. Se A é train de posto 3 satisfazendo (1.1) com decomposição de Peirce (1.2) então este ideal exprime-se por UV $\oplus \mathrm{V}$. Voltaremos a falar deste ideal nos capítulos seguintes. Ele servirá para motivar a formulação de problemas referentes a t-álgebras, que serão tratados neste trabalho.

Denotando-se por I(A) o conjunto dos elementos idempotentes de A. (necessariamente de peso 1), é conhecido que

$$
\mathrm{I}(\mathrm{A})=\left\{\mathrm{e}_{0}=\mathrm{e}+\mathrm{u}_{0}+\lambda \mathrm{u}_{0}^{2}: \mathrm{u}_{0} \in \mathrm{U}_{\mathrm{e}}\right\}
$$

sendo $\lambda=(1-2 \gamma)^{-1}$. Ou seja, $\mathrm{I}(\mathrm{A})$ é parametrizado por $\mathrm{U}_{\epsilon}$. Também com relação a e é possível considerar a decomposição de Peirce de A, isto é,

$$
\mathrm{A}=\mathrm{Fe}_{0} \oplus \mathrm{U}_{0} \oplus \mathrm{V}_{0}
$$

sendo $U_{0}=\left\{u \in N: 2 e_{0} u=u\right\} . V_{0}=\left\{v \in N: e_{0} v=\uparrow v\right\}$. As relações entre os subespaços $\mathrm{U}_{e}$ e $\mathrm{U}_{0}, \mathrm{~V}_{e}$ e $\mathrm{V}_{0}$ são as seguintes:

$$
\begin{aligned}
& \mathrm{U}_{0}=\left\{\mathrm{u}+2 \lambda \mathrm{u}_{0} \mathrm{u}: \mathrm{u} \in \mathrm{U}_{\mathrm{e}}\right\} \\
& \mathrm{V}_{0}=\left\{\mathrm{v}-2 \lambda \mathrm{u}_{0} \mathrm{v}: \mathrm{v} \in \mathrm{V}_{\mathrm{e}}\right\}
\end{aligned}
$$

Além disso, segue de (1.8), (1.9), (1.20) e (1.21) que as aplicações lineares

$$
\begin{aligned}
& \mathrm{u} \in \mathrm{U}_{\mathrm{e}} \mapsto \mathrm{u}+2 \lambda \mathrm{u}_{0} \mathrm{u} \in \mathrm{U}_{0} \\
& \mathrm{v} \in \mathrm{V}_{\mathrm{e}} \mapsto \mathrm{v}-2 \lambda \mathrm{u}_{0} \mathrm{v} \in \mathrm{V}_{0}
\end{aligned}
$$


são bijetoras e portanto $\operatorname{dim} \mathrm{U}_{0}=\operatorname{dim} \mathrm{U}_{\epsilon}$ e $\operatorname{dim} \mathrm{V}_{0}=\operatorname{dim} \mathrm{V}_{\epsilon}$. Isto permite definir para t-álgebras de posto 3 o invariante numérico tipo de $A$ como sendo o par ordenado de inteiros não negativos $\left(1+\operatorname{dim} \mathrm{U}_{e}\right.$, $\operatorname{dim} \mathrm{V}_{e}$ ). A classe de todas as t-álgebras de posto 3 pode ser subdividida em subclasses formadas pelas álgebras de mesmo tipo. Duas álgebras pertencentes a duas subclasses distintas certamente não são isomorfas. Elas realizam portanto uma pré-classificação das t-álgebras de posto 3 . Para melhor visualizar este fato, costuma-se olhar para o "triângulo de tipos" a seguir:

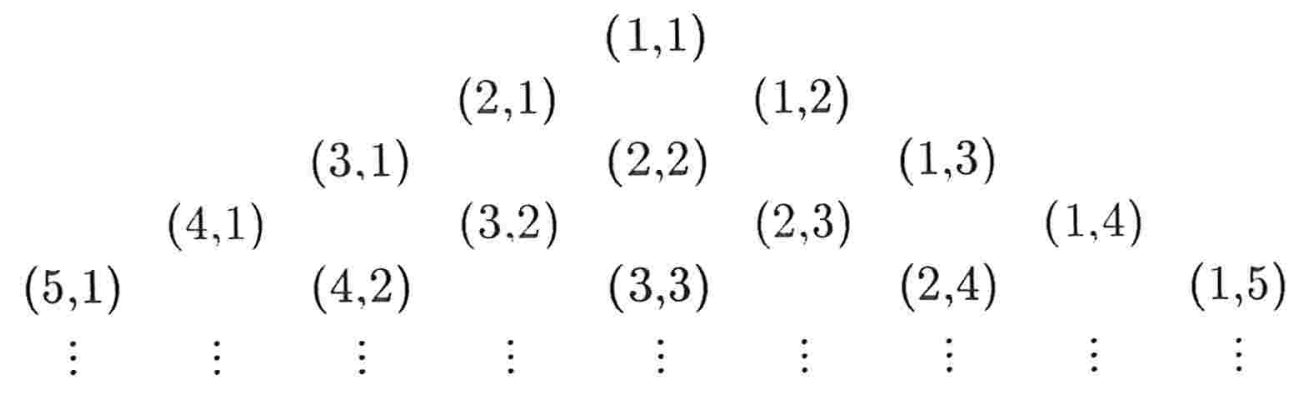

As álgebras de tipo $(1+\mathrm{n}, 0)$ foram omitidas do "triângulo" pois satisfazem a t-equação $\mathrm{x}^{2}-\omega(\mathrm{x}) \mathrm{x}=0$ e portanto têm posto 2 e sua estrutura é bem conhecida. De fato, sua tábua de multiplicação tem a forma: $\mathrm{e}^{2}=$ $=\mathrm{e}, 2 \mathrm{eu}_{i}=\mathrm{u}_{i}(\mathrm{i}=1,2, \ldots, \mathrm{n})$, sendo nulos os demais produtos. Nos artigos [6] e [7] foram descritas as t-álgebras de tipos $(1, n),(n+1,0)$, $(2, \mathrm{n}-1),(\mathrm{n}, 1),(3, \mathrm{n}-2)$, o que garante a classificação das t-álgebras de posto 3 e dimensão $\leq 5$.

\subsection{Os subespaços $p(U, V)$ : problemas}

Para simplificar as notações, daqui para frente denotaremos $\mathrm{U}_{e}$ e $\mathrm{V}_{\epsilon}$ por $\mathrm{U}$ e $\mathrm{V}$, respectivamente, sempre que isto não acarretar imprecisão de linguagem. Quando trabalharmos com dois idempotentes $e$ e $e_{0}$ usaremos 
as notações $\mathrm{U}, \mathrm{V}, \mathrm{U}_{0}$ e $\mathrm{V}_{0}$ para os correspondentes subespaços de Peirce, de acordo com (1.20) e (1.21).

Esta seção está dedicada à formulação de uma série de questões envolvendo certos subespaços de uma t-álgebra de posto 3 satisfazendo a equação (1.1) com $2 \gamma \neq 1$. Estes problemas vão surgindo de uma observação cuidadosa da decomposição de Peirce (1.2) e das relações que a seguem. Achamos que a solução destes problemas, mesmo que parcial, pode contribuir para um melhor conhecimento destas álgebras.

Seja então $(\mathrm{A}, \omega)$ uma t-álgebra de posto 3 , satisfazendo a equação (1.1) com $2 \gamma \neq 1$ e seja (1.2) sua decomposição de Peirce relativa a um idempotente genérico $e$. Denotemos por $\mathrm{m}(\mathrm{U}, \mathrm{V})$ todo subespaço vetorial de $\mathrm{A}$ (em realidade, de $\mathrm{N}=$ ker $\omega$ ) obtido multiplicando, de maneira não associativa, os subespaços U e V. Tais subespaços serão chamados de monômios de Peirce (ou P-monômios) de A. Por exemplo, U, V, U², $\mathrm{UV}, \mathrm{U}^{3}, \mathrm{U}^{3} \mathrm{~V},(\mathrm{UV}) \mathrm{V}, \mathrm{U}[(\mathrm{UV}) \mathrm{V}],[(\mathrm{UV}) \mathrm{V}] \mathrm{V}$, etc, ... são P-monômios. $\mathrm{O}$ número de fatores em $\mathrm{m}(\mathrm{U}, \mathrm{V})$ é dito o grau de $\mathrm{m}(\mathrm{U}, \mathrm{V})$. Podemos também considerar o grau em $\mathrm{U}$ e em $\mathrm{V}$ separadamente.

Decorre facilmente das equações (1.8), (1.9) e (1.10) que todo P-monômio está contido em U ou em V (mas não em ambos, exceto se for zero). Em particular, por exemplo, se $\mathrm{m}(\mathrm{U}, \mathrm{V}) \subseteq \mathrm{U}$ e A é de tipo $(1+\mathrm{r}, \mathrm{s})$ então $\operatorname{dim} \mathrm{m}(\mathrm{U}, \mathrm{V}) \leq \mathrm{r}$. O primeiro problema é uma pergunta natural:

Problema 1: É possível determinar se $\mathrm{m}(\mathrm{U}, \mathrm{V})$ está contido em $\mathrm{U}$ ou em $\mathrm{V}$, em função do grau em U e V de $\mathrm{m}(\mathrm{U}, \mathrm{V})$ ?

Esta questão é respondida no Lema 1.1, que estabelece uma condição necessária e suficiente para que um dado P-monômio de grau arbitrário esteja contido em U.

Consideremos agora todos os $\mathrm{P}$-monômios $\mathrm{m}(\mathrm{U}, \mathrm{V})$ onde fixamos a priori o número máximo de fatores $U$ e $V$. Uma parte deles está contida em $U$ e outra em V. 
Problema 2: Estabelecer as relações de inclusão entre os P-monômios contidos em U (respectivamente, em V).

A título de exemplo, os P-monómios não nulos contidos em U e de grau $\leq 4$ são:

$$
\mathrm{U}, \mathrm{UV}, \mathrm{U}^{3},(\mathrm{UV}) \mathrm{V}, \mathrm{U}[\mathrm{U}(\mathrm{UV})], \mathrm{U}^{3} \mathrm{~V}=\mathrm{U}^{2}(\mathrm{UV}),[(\mathrm{UV}) \mathrm{V}] \mathrm{V}
$$

Da mesma forma, os P-monômios não nulos contidos em $\mathrm{V}$ e de grau $\leq 4$ são:

$$
\mathrm{V}, \mathrm{U}^{2}, \mathrm{U}(\mathrm{UV}), \mathrm{U}[(\mathrm{UV}) \mathrm{V}]=(\mathrm{UV})^{2}, \mathrm{U}^{4}
$$

As relações de inclusão entre tais subespaços, que seguem diretamente das equações (1.8) a (1.12), são dadas nos diagramas a seguir:

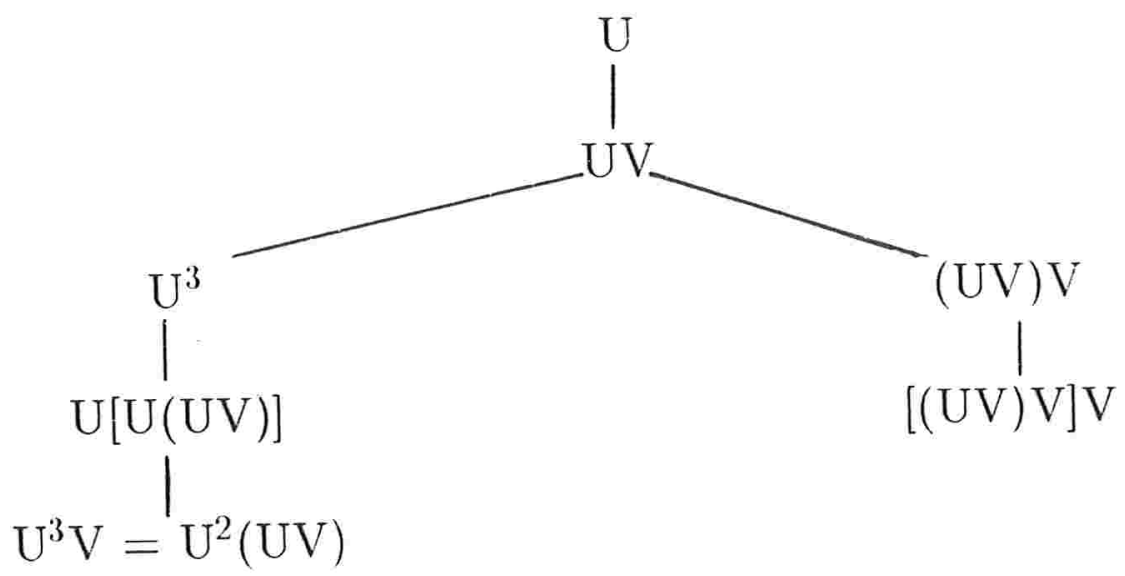




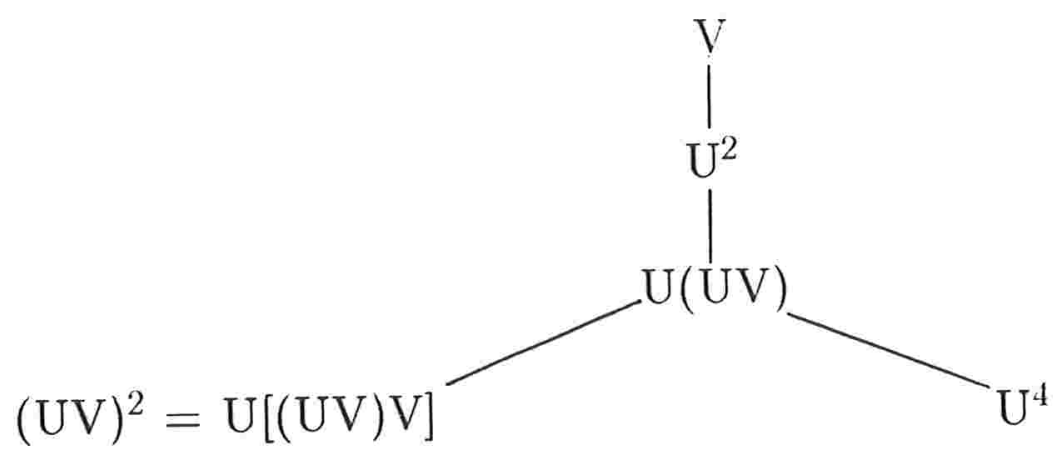

Por exemplo, a inclusão $\mathrm{U}[\mathrm{U}(\mathrm{UV})] \subseteq \mathrm{U}^{3}$ do primeiro diagrama vem do fato de que $\mathrm{UV} \subseteq \mathrm{U}$. A análise destes conjuntos parcialmente ordenados de subespaços ajuda a entender a estrutura das t-álgebras de posto 3 . A solução deste problema, em cada caso concreto, pode ser feita usando as equações (1.6), (1.7), as inclusões (1.8) a (1.12) e as igualdades (1.13) a (1.18). Como caso particular deste problema, aparece a questão de saber quando dois $\mathrm{P}$-monômios são genericamente iguais. Já observamos que $\mathrm{U}[(\mathrm{UV}) \mathrm{V}]=(\mathrm{UV})^{2}$ e também que $\mathrm{U}^{3} \mathrm{~V}=\mathrm{U}^{2}(\mathrm{UV})$. A demonstração destas igualdades será feita mais adiante, no Lema 3.1. Ao estabelecermos igualdades desta natureza estamos mostrando que as t-álgebras satisfazem formas (muito) fracas de associatividade, que são igualdades envolvendo subespaços e não elementos.

Passemos agora a subespaços mais gerais, que são obtidos por soma de P-monômios. Estes serão chamados P-subespaços ou subespaços de Peirce de A. Eles têm a forma $\mathrm{p}(\mathrm{U}, \mathrm{V})=\sum \mathrm{m}(\mathrm{U}, \mathrm{V})$. A soma poderá ser direta ou não. Por exemplo, $\mathrm{p}(\mathrm{U}, \mathrm{V})=\mathrm{UV} \oplus \mathrm{V}$ é um P-subespaço, o mesmo ocorrendo com $\mathrm{N}=\mathrm{U} \oplus \mathrm{V}$.

Problema 3: Quais dos P-subespaços $\mathrm{p}(\mathrm{U}, \mathrm{V})$ são invariantes em relação a uma mudança arbitrária de idempotentes?

Com as notações da seção anterior, queremos que se tenha sempre $\mathrm{p}(\mathrm{U}, \mathrm{V})=\mathrm{p}\left(\mathrm{U}_{0}, \mathrm{~V}_{0}\right)$. Um exemplo importante deste caso é $\mathrm{p}(\mathrm{U}, \mathrm{V})=$ $=\mathrm{UV} \oplus \mathrm{V}$ que é o ideal de $\mathrm{A}$ gerado pelos elementos $\mathrm{a}^{2}-\omega(\mathrm{a}) \mathrm{a}$, a $\in$ 
$\in$ A. Esta maneira de descrevê-lo já mostra que UV $\oplus \mathrm{V}=\mathrm{U}_{0} \mathrm{~V}_{0} \oplus$ $\oplus \mathrm{V}_{0}$. Também temos $\mathrm{N}=\mathrm{U} \odot \mathrm{V}=\mathrm{U}_{0} \oplus \mathrm{V}_{0}$ como outro exemplo desta situação.

O problema seguinte propõe uma questão um pouco mais fraca.

Problema 4: Quais dos P-subespaços $\mathrm{p}(\mathrm{U}, \mathrm{V})$ têm dimensão invariante em relação a uma mudança arbitrária de idempotentes?

Aqui queremos que $\operatorname{dim} \mathrm{p}(\mathrm{U}, \mathrm{V})=\operatorname{dim} \mathrm{p}\left(\mathrm{U}_{0}, \mathrm{~V}_{0}\right)$, com as notações da seção anterior. É claro que toda solução do Problema 3 é também solução do Problema 4.

Neste trabalho, restringimos nossos estudos aos P-monômios e P-subespaços de grau $\leq 4$, num total de 89 subespaços (apenas em alguns casos isolados consideramos graus arbitrários). No Capítulo 5, respondendo às questões dadas nos problemas 3 e 4 , mostramos que os 89 P-subespaços de grau $\leq 4$ podem ser agrupados em dois conjuntos: o Grupo I, que contém 24 subespaços que provamos serem invariantes por mudança de idempotentes e o Grupo II, contendo 65 P-subespaços que têm dimensão invariante por mudança de idempotentes

Problema 5: Sabendo que $\operatorname{dim} p(U, V)=\operatorname{dim} p\left(U_{0}, V_{0}\right)$ é possível encontrar uma aplicação linear bijetora "natural" (como ocorreu em (1.20) e (1.21)) entre $\mathrm{p}(\mathrm{U}, \mathrm{V})$ e $\mathrm{p}\left(\mathrm{U}_{0}, \mathrm{~V}_{0}\right)$ ?

Ainda no Capítulo 5, respondemos parcialmente a esta questão (veja Lemas $5.10,5.11,5.12$ e 5.13$)$.

Observemos que $\mathrm{p}(\mathrm{U}, \mathrm{V})=\mathrm{UV} \oplus \mathrm{V}$ é um ideal de $\mathrm{A}$ e que $\mathrm{m}(\mathrm{U}, \mathrm{V})=$ $=\mathrm{V}$ não é um ideal de A. A pergunta natural é então a seguinte:

Problema 6: Quais dos P-subespaços p(U, V) são ideais de A?

Outros exemplos de ideais são $\mathrm{N}=\mathrm{U} \oplus \mathrm{V}, \mathrm{U} \oplus \mathrm{U}^{2}$ e $\mathrm{N}^{i}=(\mathrm{U} \oplus \mathrm{V})^{i}$. 
A questão colocada no problema 6 se desdobra naturalmente em várias outras. Por exemplo, o produto de dois ideais da forma $p(U, V)$ é ainda um ideal? Ideais que se definem naturalmente em A, em geral através de um conjunto de geradores ou alguma propriedade a ser satisfeita por seus elementos, são ideais da forma $\mathrm{p}(\mathrm{U}, \mathrm{V})$ ? Um exemplo importante desta questão é o caso do ideal $\mathrm{L}$, definido por $\mathrm{L}=\{\mathrm{u} \in \mathrm{U}: \mathrm{uU}=0\}$. É êle $\mathrm{um}$ ideal polinomial? Se um subespaço $\mathrm{p}(\mathrm{U}, \mathrm{V})$ não é um ideal de $\mathrm{A}$, como exprimir o ideal gerado por $\mathrm{p}(\mathrm{U}, \mathrm{V})$ ?

O Teorema 1 do Capítulo 6 garante que todos os P-subespaços do Grupo I são ideais de A. Além disso, ainda neste capítulo, descrevemos 4 exemplos que mostram que, dos $65 \mathrm{P}$-subespaços que compõem o Grupo II, 61 não são ideais de $\mathrm{A}$ (sobre os outros 4 nada sabemos dizer até então). Ainda respondendo ao problema 6 , no Lema 6.10 mostramos que o produto de dois ideais da forma $\mathrm{p}(\mathrm{U}, \mathrm{V})$ é um ideal de A. O Exemplo 31 mostra que o ideal L acima definido não é polinomial e finalmente, nos Lemas 6.1 a 6.8, calculamos os $\mathrm{P}$-subespaços gerados pelos monômios de Peirce de grau $\leq 4$ (que já sabemos não serem ideais de $\mathrm{A}$ ).

Problema 7: É possível estimar a dimensão mínima da t-álgebra para a qual um determinado P-monômio é não nulo?

Conforme veremos, podemos obter respostas bem precisas neste problema, para subespaços monomiais de grau não muito elevado.

Problema 8: Fixado um subconjunto $S$ de $\mathrm{U}$, com $0 \in \mathrm{S}$, quais são os P-subespaços $\mathrm{p}(\mathrm{U}, \mathrm{V})$ tais que $\mathrm{p}(\mathrm{U}, \mathrm{V})=\mathrm{p}\left(\mathrm{U}_{0}, \mathrm{~V}_{0}\right)$ para todo $\mathrm{u}_{0} \in \mathrm{S}$ ? E quais são aqueles em que $\operatorname{dim} p(U, V)=\operatorname{dim} p\left(U_{0}, V_{0}\right)$ para todo $u_{0} \in$ $\in \mathrm{S}$ ?

No capítulo 7 respondemos a essa questão para $\mathrm{S}=\mathrm{F}\left(\mathrm{u}_{0} \mathrm{v}_{0}\right)$, sendo $\mathrm{u}_{0} \in$ $\in \mathrm{U}$ e $\mathrm{v}_{0} \in \mathrm{V}$. 
Problema 9: É o problema inverso do anterior: fixado um P-subespaço $\mathrm{p}(\mathrm{U}, \mathrm{V})$, é possível determinar o subconjunto $S$ de $\mathrm{U}$ tal que $\mathrm{p}(\mathrm{U}, \mathrm{V})=$ $=\mathrm{p}\left(\mathrm{U}_{0}, \mathrm{~V}_{0}\right)$ para todo $\mathrm{u}_{0} \in \mathrm{S}$ ?

Veremos, no capítulo 7, que é possível responder a essa questão para alguns $\mathrm{P}$-subespaços.

O objetivo principal desse trabalho é estudar a invariância dos P-subespaços, ou pelo menos, a invariância de suas dimensões (Problemas 3 e 4). O trabalho desenvolve-se caso a caso, pois não pudemos encontrar métodos gerais para tratar os problemas propostos.

O Lema seguinte é uma resposta ao Problema 1.

Lema 1.1 Sejam $A=F e \oplus U \oplus V$ uma t-álgebra de posto $3 e$ $0 \neq m(U, V)$ um monômio de Peirce de $A$ contendo pelo menos um. fator $U$. Uma condição necessária e suficiente para que $m(U, V)$ esteja contido em $U$ é que o número de fatores $U$ 's em $m(U, V)$ seja ímpar.

Prova: Se $m(U, V)$ tem grau 1, então $m(U, V) \subseteq U$ se e somente se $\mathrm{m}(\mathrm{U}, \mathrm{V})=\mathrm{U}$ e neste caso o número de fatores $\mathrm{U}$ 's em $\mathrm{m}(\mathrm{U}, \mathrm{V})$ é ímpar. Supondo que $\mathrm{m}(\mathrm{U}, \mathrm{V})$ tenha grau 2, temos por (1.8), (1.9) e (1.10), $\mathrm{m}(\mathrm{U}, \mathrm{V}) \subseteq \mathrm{U}$ se e somente se $\mathrm{m}(\mathrm{U}, \mathrm{V})=\mathrm{UV}$ e neste caso também o número de fatores $U$ 's em $\mathrm{m}(\mathrm{U}, \mathrm{V})$ é ímpar. Supondo agora que o grau de $\mathrm{m}(\mathrm{U}, \mathrm{V})$ seja 3 , então $\mathrm{m}(\mathrm{U}, \mathrm{V}) \subseteq \mathrm{U}$ se e somente se $\mathrm{m}(\mathrm{U}, \mathrm{V})=\mathrm{U}^{3}$ ou $\mathrm{m}(\mathrm{U}, \mathrm{V})=(\mathrm{UV}) \mathrm{V}$, por (1.8) e (1.9), e aqui também o número de fatores U's em $\mathrm{m}(\mathrm{U}, \mathrm{V})$ é ímpar, o que mostra que a tese é verdadeira para $n=1,2,3$. Suponhamos agora por indução que a propriedade seja verdadeira para todo $3<k<n+1$ e consideremos $\mathrm{m}(\mathrm{U}, \mathrm{V})$ de grau $n+1$. O subespaço $\mathrm{m}(\mathrm{U}, \mathrm{V})$ pode ser decomposto na forma $\mathrm{m}(\mathrm{U}, \mathrm{V})=\left(\mathrm{m}_{1} \mathrm{~m}_{2}\right) \mathrm{m}_{3}$, onde $\mathrm{m}_{i}=\mathrm{m}_{i}(\mathrm{U}, \mathrm{V})$ são subespaços monomiais de graus menores que $n+1$. Assim $m(\mathrm{U} . \mathrm{V}) \subseteq \mathrm{U}$ se e somente se ocorrer um dos dois casos (i) $\mathrm{m}_{1} \subseteq \mathrm{U} . \mathrm{m}_{2} . \mathrm{m}_{3} \subseteq \mathrm{V}$ ou (ii) $\mathrm{m}_{1}, \mathrm{~m}_{2}$. 
$\mathrm{m}_{3} \subseteq \mathrm{U}$. Observando que em ambos os casos temos grau $\mathrm{m}_{i}<$ $<$ grau $\mathrm{m}(\mathrm{U}, \mathrm{V})<n+1$, pela hipótese de indução no primeiro caso. temos $\mathrm{m}_{1} \subseteq \mathrm{U}, \mathrm{m}_{2}, \mathrm{~m}_{3} \subseteq \mathrm{V}$ se e somente se o número de U's em $\mathrm{m}_{1}$ é ímpar e o número de U's em $\mathrm{m}_{2}, \mathrm{~m}_{3}$ é par e isto é equivalente a dizer que o número de U's em $\mathrm{m}(\mathrm{U}, \mathrm{V})$ é ímpar. No segundo caso, temos $\mathrm{m}_{1}$, $\mathrm{m}_{2}, \mathrm{~m}_{3} \subseteq \mathrm{U}$ se e somente se em cada um dos P-monômios $\mathrm{m}_{1}, \mathrm{~m}_{2}, \mathrm{~m}_{3} \mathrm{o}$ número de U's em $\mathrm{m}(\mathrm{U}, \mathrm{V})$ é ímpar, o que é equivalente a que o número de fatores U's em m(U, V) é ímpar. Assim o resultado é válido para $n+1$, donde segue o resultado enunciado.

Corolário 1.1.1 Com as notações e hipóteses do Lema 1.1, temos que $m(U, V) \subseteq V$ se e somente se o número de fatores $U$ 's em $m(U, V)$ é par. 


\section{Capítulo 2}

\section{Sobre os subespaços $\mathbf{U}^{n} \oplus \mathbf{U}^{n+1}$}

Neste capítulo vamos construir uma cadeia decrescente de P-subespaços da t-álgebra A, dependendo do subespaço U, todos invariantes por mudança de idempotentes. Além disso, todos são ideais de A. Esta cadeia de ideais provavelmente será importante no estudo da estrutura das t-álgebras de posto 3 .

\subsection{Invariância da dimensão de $\mathrm{U}^{n}$}

Consideremos A uma t-álgebra de posto 3 satisfazendo a equação $\mathrm{x}^{3}-(1+\gamma) \omega(\mathrm{x}) \mathrm{x}^{2}+\gamma \omega(\mathrm{x})^{2} \mathrm{x}=0$, com $2 \gamma \neq 1$ e fixemos uma decomposição de Peirce $\mathrm{A}=\mathrm{Fe} \oplus \mathrm{U} \oplus \mathrm{V}$, relativa a um idempotente e $\in \mathrm{A}$. Lembremos que o subespaço $U^{k}$ é definido por $U^{1}=U$ e $U^{k+1}=U^{k} U$. Resulta de (1.11) e (1.12) que $\mathrm{U}^{k}$ está contido alternadamente em U e V. Para tornar a linguagem mais agradável, simplificamos às vezes a expressão "invariante por mudança de idempotentes" para "invariante".

Lema 2.1 Com as notações acima, temos $U^{n} \subseteq U^{n-2}$ para todo n. $\geq 3$.

Prova: Teremos dois casos a considerar, a saber $n$ par e $n$ ímpar. Supondo $n$ ímpar, digamos, $n=2 k+1$, queremos mostrar que $\mathrm{U}^{2 k+1} \subseteq$ $\subseteq \mathrm{U}^{2 k-1}$. A prova será feita por indução sobre $k$. Temos que $\mathrm{U}^{3}=\mathrm{U}^{2} \mathrm{U} \subseteq$ $\subseteq \mathrm{VU} \subseteq \mathrm{U}$. o que mostra que o resultado é verdadeiro para $k=1$. Supo- 
nhamos, agora, que o resultado seja verdadeiro para $k-1$, isto é. $\mathrm{U}^{2 k-1} \subseteq \mathrm{U}^{2 k-3}$, para $k \geq 2$, e provemos que vale a inclusão para $k$. Temos $\mathrm{U}^{2 k+1}=\mathrm{U}^{2 k} \mathrm{U}=\left(\mathrm{U}^{2 k-1} \mathrm{U}\right) \mathrm{U} \subseteq\left(\mathrm{U}^{2 k-3} \mathrm{U}\right) \mathrm{U}=\mathrm{U}^{2 k-2} \mathrm{U}=\mathrm{U}^{2 k-1}$ e portanto tem-se a inclusão $\mathrm{U}^{n} \subseteq \mathrm{U}^{n-2}$ para todo $n$ ímpar. Suponhamos agora que $n$ seja par. Nesse caso, $n=2 k$ e queremos mostrar que $\mathrm{U}^{2 k} \subseteq \mathrm{U}^{2 k-2}$. Temos $\mathrm{U}^{2 k}=\mathrm{U}^{2 k-1} \mathrm{U} \subseteq \mathrm{U}^{2 k-3} \mathrm{U}=\mathrm{U}^{2 k-2}$, o que mostra que $\mathrm{U}^{2 k} \subseteq \mathrm{U}^{2 k-2}$ e que, portanto, $\mathrm{U}^{n} \subseteq \mathrm{U}^{n-2}$ para todo $n$ par, o que completa a prova do resultado enunciado.

Corolário 2.1.1 As potências pares (respectivamente, ímpares), do subespaço $U$ formam uma cadeia decrescente de subespaços de $V$ (respectivamente, $U$ ).

Lema 2.2 O P-subespaço $U \oplus U^{2}$ é um ideal de A, que é invariante por mudança de idempotentes.

Prova: Provemos primeiro que $\mathrm{U} \oplus \mathrm{U}^{2}$ é um ideal de A. Para isso, seja $\mathrm{x}=\mathrm{a}+\mathrm{b} \in \mathrm{U} \oplus \mathrm{U}^{2}$, com $\mathrm{a} \in \mathrm{U}$ e $\mathrm{b} \in \mathrm{U}^{2}$. Considerando os elementos $\mathrm{e}, \mathrm{u} \in \mathrm{U}, \mathrm{v} \in \mathrm{V}$ temos:

$$
\begin{gathered}
\mathrm{ex}=\mathrm{ea}+\mathrm{eb}=\frac{1}{2} \mathrm{a}+\gamma \mathrm{b} \in \mathrm{U} \oplus \mathrm{U}^{2} \\
\mathrm{ux}=\mathrm{ua}+\mathrm{ub} \in \mathrm{U}^{2}+\mathrm{UV} \subseteq \mathrm{U}^{2} \ominus \mathrm{U}=\mathrm{U} \ominus \mathrm{U}^{2} \\
\mathrm{vx}=\mathrm{av}+\mathrm{bv}=\mathrm{av} \in \mathrm{UV} \subseteq \mathrm{U} \subseteq \mathrm{U} \ominus \mathrm{U}^{2}
\end{gathered}
$$

o que mostra que o subespaço $U \oplus \mathrm{U}^{2}$ é um ideal de $\mathrm{A}$. Provemos agora que $\mathrm{U}_{0} \oplus \mathrm{U}_{0}^{2}=\mathrm{U} \oplus \mathrm{U}^{2}$, para todo idempotente $\mathrm{e}_{0}$ de $A$. Sendo $\mathrm{e}_{0} \mathrm{um}$ idempotente de A, então por (1.19), $\mathrm{e}_{0}=\mathrm{e}+\mathrm{u}_{0}+\lambda \mathrm{u}_{0}^{2}$, com $\mathrm{u}_{0} \in \mathrm{U}$ e $\lambda=(1-2 \gamma)^{-1} \in \mathrm{F}$. Observamos de (1.20) que $\mathrm{U}_{0}=\left\{\mathrm{u}+2 \lambda \mathrm{u}_{0} \mathrm{u}: \mathrm{u} \in\right.$ $\in \mathrm{U}\} \subseteq \mathrm{U} \ominus \mathrm{U}^{2}$. Quanto a $\mathrm{U}_{0}^{2}$. tomemos um de seus geradores aditivos $\left(\mathrm{u}_{1}+2 \lambda \mathrm{u}_{0} \mathrm{u}_{1}\right)\left(\mathrm{u}_{2}+2 \lambda \mathrm{u}_{0} \mathrm{u}_{2}\right)=\mathrm{u}_{1} \mathrm{u}_{2}+2 \lambda \mathrm{u}_{1}\left(\mathrm{u}_{0} \mathrm{u}_{2}\right)+2 \lambda \mathrm{u}_{2}\left(\mathrm{u}_{0} \mathrm{u}_{1}\right)+$ 
$+4 \lambda^{2}\left(\mathrm{u}_{0} \mathrm{u}_{1}\right)\left(\mathrm{u}_{0} \mathrm{u}_{2}\right)$. De $\mathrm{J}\left(\mathrm{u}_{0}, \mathrm{u}_{1}, \mathrm{u}_{2}\right)=0$, obtemos $\mathrm{u}_{1}\left(\mathrm{u}_{0} \mathrm{u}_{2}\right)+\mathrm{u}_{2}\left(\mathrm{u}_{0} \mathrm{u}_{1}\right)=$ $=-\mathrm{u}_{0}\left(\mathrm{u}_{1} \mathrm{u}_{2}\right)$. Além disso, como $\left(\mathrm{u}_{0} \mathrm{u}_{1}\right)\left(\mathrm{u}_{0} \mathrm{u}_{2}\right) \in \mathrm{V}^{2}=0$ e $\mathrm{U}^{3} \subseteq \mathrm{U}$, segue que $\left(\mathrm{u}_{1}+2 \lambda \mathrm{u}_{0} \mathrm{u}_{1}\right)\left(\mathrm{u}_{2}+2 \lambda \mathrm{u}_{0} \mathrm{u}_{2}\right)=\mathrm{u}_{1} \mathrm{u}_{2}-2 \lambda \mathrm{u}_{0}\left(\mathrm{u}_{1} \mathrm{u}_{2}\right) \in \mathrm{U}^{2} \oplus \mathrm{U}^{3} \subseteq \mathrm{U}^{2} \oplus \mathrm{U}=$ $=\mathrm{U} \oplus \mathrm{U}^{2}$. Por linearidade, obtemos que $\mathrm{U}_{0}^{2} \subseteq \mathrm{U} \oplus \mathrm{U}^{2}$ e como já temos $\mathrm{U}_{0} \subseteq \mathrm{U} \oplus \mathrm{U}^{2}$, segue que $\mathrm{U}_{0} \oplus \mathrm{U}_{0}^{2} \subseteq \mathrm{U} \oplus \mathrm{U}^{2}$, para todo idempotente $\mathrm{e}_{0}$ de A. Por simetria, obtemos a inclusão contrária.

Corolário 2.2.1 A dimensão do subespaço $U \oplus U^{2}$ é invariante por mudança de idempotentes.

Corolário 2.2.2 O subespaço $U^{2}$ tem dimensão invariante (isto é, $\left.\operatorname{dim} U^{2}=\operatorname{dim} U_{0}^{2}\right)$.

Prova: Segue do Corolário 2.2 .1 e do fato que dim U é invariante.

Lema 2.3 $U^{n} V=0$ se n é par e $U^{n} V \subseteq U^{n}$ se né ímpar.

Prova: Se $n$ é par, o resultado é óbvio pois $\mathrm{U}^{n} \subseteq \mathrm{V}$ e portanto $\mathrm{U}^{n} \mathrm{~V} \subseteq$ $\subseteq \mathrm{V}^{2}=0$. Suponhamos então que $n$ seja ímpar: isto é. $n=2 k+1$. Então $\mathrm{U}^{n} \mathrm{~V}=\mathrm{U}^{2 k+1} \mathrm{~V}=\left(\mathrm{U}^{2 k} \mathrm{U}\right) \mathrm{V}$ e da identidade (1.13) segue que $\mathrm{U}^{2 k}(\mathrm{UV})+$ $+\left(\mathrm{U}^{2 k} \mathrm{U}\right) \mathrm{V}=0$, uma vez que $\mathrm{U}^{2 k} \mathrm{~V} \subseteq \mathrm{V}^{2}=0$. Assim, da identidade de Jacobi aplicada a elementos de $\mathrm{U}^{2 k}, \mathrm{U}$ e $\mathrm{V}$, segue que $\mathrm{U}^{n} \mathrm{~V}=\left(\mathrm{U}^{2 k} \mathrm{U}\right) \mathrm{V}=$ $=\mathrm{U}^{2 k}(\mathrm{UV}) \subseteq \mathrm{U}^{2 k} \mathrm{U}=\mathrm{U}^{2 k+1}=\mathrm{U}^{n}$.

Lema 2.4 Para todo $n \geq 1$, o subespaço $U^{n} \ominus U^{n+1}$ é um ideal de A.

Prova: Teremos dois casos a considerar, a saber, $n$ par e $n$ ímpar. Supondo $n$ ímpar, digamos $n=2 k+1$, devemos mostrar que $\mathrm{U}^{2 k+1}$ $\oplus \mathrm{U}^{2 k+2}$ é ideal de A. Para isso, consideremos $\mathrm{x}=\mathrm{a}+\mathrm{b}$, com $\mathrm{a} \in \mathrm{U}^{2 k+1}$ $\mathrm{e} \mathrm{b} \in \mathrm{U}^{2 k+2}$. Tomando os elementos e, $\mathrm{u} \in \mathrm{U}, \mathrm{v} \in \mathrm{V}$, temos:

(i) $\mathrm{ex}=\mathrm{ea}+\mathrm{eb}=\frac{1}{2} \mathrm{a}+\gamma \mathrm{b} \in \mathrm{U}^{2 k+1} \ominus \mathrm{U}^{2 k+2}$. 
(ii) $\mathrm{ux}=\mathrm{ua}+\mathrm{ub} \in \mathrm{u} \mathrm{U}^{2 k+1}+\mathrm{u} \mathrm{U}^{2 k+2} \subseteq \mathrm{U}^{2 k+2} \odot \mathrm{U}^{2 k+3} \subseteq \mathrm{U}^{2 k+2} \Theta$ $\oplus \mathrm{U}^{2 k+1}$, pelo Lema 2.1 .

(iii) $\mathrm{vx}=\mathrm{va}+\mathrm{vb}=\mathrm{va} \in \mathrm{U}^{2 k+1}$, pois $\mathrm{vb} \in \mathrm{VU}^{2 k+2} \subseteq \mathrm{V}^{2}=0$ e, pelo Lema $2.3, \mathrm{U}^{2 k+1} \mathrm{~V} \subseteq \mathrm{U}^{2 k+1}$.

Conclui-se, portanto, que $\mathrm{vx} \in \mathrm{vU}^{2 k+1} \subseteq \mathrm{U}^{2 k+1} \subseteq \mathrm{U}^{2 k+1} \oplus \mathrm{U}^{2 k+2}$, o que completa a prova de que $\mathrm{U}^{n} \oplus \mathrm{U}^{n+1}$ é um ideal de $\mathrm{A}$, se $n$ é ímpar.

Suponhamos agora que $n$ seja par. Nesse caso $n=2 k$ e queremos mostrar que $\mathrm{U}^{2 k} \oplus \mathrm{U}^{2 k+1}$ é ideal de $\mathrm{A}$. Seja $\mathrm{x}=\mathrm{a}+\mathrm{b}$, sendo $\mathrm{a} \in \mathrm{U}^{2 k}$ e $\mathrm{b} \in \mathrm{U}^{2 k+1}$. Considerando e, $\mathrm{u} \in \mathrm{U}, \mathrm{v} \in \mathrm{V}$, temos:

(i) $\mathrm{ex}=\mathrm{ea}+\mathrm{eb}=\gamma \mathrm{a}+\frac{1}{2} \mathrm{~b} \in \mathrm{U}^{2 k} \oplus \mathrm{U}^{2 k+1}$.

(ii) $\mathrm{ux}=\mathrm{ua}+\mathrm{ub} \in \mathrm{u} \mathrm{U}^{2 k}+\mathrm{u} \mathrm{U}^{2 k+1} \subseteq \mathrm{U}^{2 k+1} \oplus \mathrm{uU}^{2 k+1} \subseteq \mathrm{U}^{2 k+1} \oplus$ $\oplus \mathrm{uU}^{2 k-1} \subseteq \mathrm{U}^{2 k+1} \oplus \mathrm{U}^{2 k}$, pois, pelo Lema $2.1, \mathrm{U}^{2 k+1} \subseteq \mathrm{U}^{2 k-1}$.

(iii) $\mathrm{vx}=\mathrm{va}+\mathrm{vb}=\mathrm{vb} \in \mathrm{U}^{2 k+1}$, pois va $\in \mathrm{VU}^{2 k} \subseteq \mathrm{V}^{2}=0$ e $\mathrm{vU}^{2 k+1} \subseteq$ $\subseteq \mathrm{U}^{2 k+1}$, pelo Lema 2.3.

Logo, vx $\in \mathrm{U}^{2 k+1} \subseteq \mathrm{U}^{2 k} \oplus \mathrm{U}^{2 k+1}$, o que completa a prova de que $\mathrm{U}^{n} \oplus$ $\oplus \mathrm{U}^{n+1}$ é ideal de $\mathrm{A}$, se $n$ é par. Portanto, $\mathrm{U}^{n} \oplus \mathrm{U}^{n+1}$ é ideal de A, para todo $n \geq 1$.

Lema 2.5 Sejam $A=F e \oplus U \oplus V=F e_{0} \oplus U_{0} \oplus V_{0}$ duas decomposiçôes de Peirce de uma t-álgebra de posto $3 \mathrm{~A}$, relativas aos idempotentes e e $e_{0}=e+u_{0}+\lambda u_{0}^{2}$, respectivamente. Então, para todo $n \geq 1$, $U^{n} U_{0} \subseteq U^{n+1} \Theta U^{n+2}$.

Prova: Um gerador aditivo de $\mathrm{U}_{0}$ é da forma $\mathrm{u}_{1}=\mathrm{u}+2 \lambda \mathrm{u}_{0} \mathrm{u}$, com $\mathrm{u} \in$ $\in \mathrm{U}$. Logo, $\mathrm{U}^{n} \mathrm{u}_{1} \subseteq \mathrm{U}^{n} \mathrm{u} \oplus \mathrm{U}^{n}\left(\mathrm{u}_{0} \mathrm{u}\right) \subseteq \mathrm{U}^{n+1} \oplus \mathrm{U}^{n}\left(\mathrm{u}_{0} \mathrm{u}\right)$. Se $n$ é par, pelo Lema 2.3, $\mathrm{U}^{n}\left(\mathrm{u}_{0} \mathrm{u}\right)=0$ e portanto $\mathrm{U}^{n} \mathrm{u}_{1} \subseteq \mathrm{U}^{n+1} \subseteq \mathrm{U}^{n+1} \oplus \mathrm{U}^{n+2}$. Por linearidade, obtemos $\mathrm{U}^{n} \mathrm{U}_{0} \subseteq \mathrm{U}^{n+1} \oplus \mathrm{U}^{n+2}$. Se $n$ é ímpar, temos que $\mathrm{U}^{n}\left(\mathrm{u}_{0} \mathrm{u}\right) \subseteq \mathrm{U}^{n} \mathrm{U}^{2} \subseteq \mathrm{U}^{n+2}$ (pois, como observamos no Capítulo $1, \mathrm{X}^{i} \mathrm{X}^{j} \subseteq$ $\subseteq \mathrm{X}^{i+j}$, para todo subespaço $X$ de A). Dessa forma, $\mathrm{U}^{n} \mathrm{u}_{1} \subseteq \mathrm{U}^{n+2} \subseteq$ $\subseteq \mathrm{U}^{n+1} \oplus \mathrm{U}^{n+2}$ e novamente por linearidade segue que $\mathrm{U}^{n} \mathrm{U}_{0} \subseteq \mathrm{U}^{n+1} \ominus$ $\oplus \mathrm{U}^{n+2}$. 
Lema 2.6 Para todo $n \geq 1$, o ideal $U^{n} \oplus U^{n+1}$ é invariante por mudança de idempotentes.

Prova: Devemos mostrar que $U_{0}^{n} \oplus U_{0}^{n+1}=U^{n} \oplus U^{n+1}$ para todo $n \geq 1$ e para todo idempotente $e_{0}$ de $\mathrm{A}$. A prova será feita por indução sobre $n$. Do Lema 2.2, temos que $\mathrm{U}_{0} \oplus \mathrm{U}_{0}^{2}=\mathrm{U} \oplus \mathrm{U}^{2}$, sendo portanto a afirmação verdadeira para $n=1$. Suponhamos, agora, que o resultado seja verdadeiro para $n=k-1$, isto é, $\mathrm{U}_{0}^{k-1} \oplus \mathrm{U}_{0}^{k}=\mathrm{U}^{k-1} \oplus \mathrm{U}^{k} \mathrm{e}$ provemos que é verdadeiro para $n=k \geq 2$. Temos que $\mathrm{U}_{0}^{k} \oplus \mathrm{U}_{0}^{k+1} \subseteq$ $\subseteq\left[\mathrm{U}_{0}^{k-1} \oplus \mathrm{U}_{0}^{k}\right] \mathrm{U}_{0}=\left[\mathrm{U}^{k-1} \oplus \mathrm{U}^{k}\right] \mathrm{U}_{0}$. Do Lema 2.5, segue que $\mathrm{U}^{k-1} \mathrm{U}_{0} \subseteq$ $\subseteq \mathrm{U}^{k} \oplus \mathrm{U}^{k+1}$ e $\mathrm{U}^{k} \mathrm{U}_{0} \subseteq \mathrm{U}^{k+1} \oplus \mathrm{U}^{k+2} \subseteq \mathrm{U}^{k+1} \oplus \mathrm{U}^{k}$ (sendo esta última inclusão consequência do Corolário 2.1.1). Assim provamos que $\mathrm{U}_{0}^{k} \oplus \mathrm{U}_{0}^{k-1} \subseteq \mathrm{U}^{k} \oplus \mathrm{U}^{k+1}$, para todo $k$. Por simetria obtém-se a inclusão contrária e portanto conclui-se que, para todo $k, \mathrm{U}_{0}^{k} \oplus \mathrm{U}_{0}^{k+1}=\mathrm{U}^{k} \oplus$ $\oplus \mathrm{U}^{k+1}$.

Corolário 2.6.1 A dimensão do subespaço $U^{n} \oplus U^{n+1}$ é invariante, para todo $n \geq 1$.

Corolário 2.6.2 dim $U^{n}$ é invariante, para todo $n \geq 1$.

Prova: A prova será feita por indução sobre $n$. Já sabemos que U e $U^{2}$ têm dimensões invariantes, o que prova o resultado para $n=1$ e $n=2$. Suponhamos, agora, que $\operatorname{dim} \mathrm{U}^{k-1}$ é invariante para $k \geq 3$ e mostremos que $\operatorname{dim} \mathrm{U}^{k}$ é invariante. Do Corolário 2.6.1, temos que $\operatorname{dim}\left(\mathrm{U}^{k-1} \oplus \mathrm{U}^{k}\right)$ é invariante e como pela hipótese de indução dim $\mathrm{U}^{k-1}$ é invariante, segue que $\operatorname{dim} \mathrm{U}^{k}$ é invariante.

Corolário 2.6.3 Os subespaços $U^{i} \ominus U^{i}, i, j \geq 1$ têm dimensão invariante sempre que i e j forem inteiros de paridades diferentes. Todos os subespaços $U^{i} \oplus V$ têm dimensão invariante se i é ímpar. 
Observação: A sequência decrescente de ideais $\mathrm{N} \supseteq \mathrm{U} \oplus \mathrm{U}^{2} \supseteq$ $\supseteq \mathrm{U}^{3} \oplus \mathrm{U}^{2} \supseteq \mathrm{U}^{3} \oplus \mathrm{U}^{4} \supseteq \ldots$ chega ao ideal nulo pois o ideal $\mathrm{N}$ é nilpotente, de acordo com o resultado de Abraham, veja [1]. 


\section{Capítulo 3}

\section{Soluções parciais do Problema 2}

Neste capítulo vamos estabelecer algumas identidades envolvendo Pmonômios em uma t-álgebra de posto 3 , com respostas (parciais) ao segundo problema colocado na Seção 3 do Capítulo 1.

\subsection{Alguns casos do Problema 2}

Conforme dissemos no Capítulo 1, o Problema 2 consiste em achar P-subespaços $\mathrm{m}_{1}(\mathrm{U}, \mathrm{V})$ e $\mathrm{m}_{2}(\mathrm{U}, \mathrm{V})$ que são genericamente iguais. Observemos que não há solução deste problema para subespaços de grau < 4. Começamos provando as igualdades entre os P-subespaços de grau 4 que aparecem em 1.3.

Lema 3.1 Sejam $A=F e \oplus U \oplus V$ uma t-álgebra de posto 3 satisfazendo (1.1) e $u, u_{1}, u_{2} \in U, v, v_{1} \in V$. Então temos as identidades $u_{1}\left[(u v) v_{1}\right]=$ $=-(u v)\left(u_{1} v_{1}\right)$ e $\left[\left(u u_{1}\right) u_{2}\right] v=-\left(u u_{1}\right)\left(u_{2} v\right)$. Como consequência, $U[(U V) V]$ $=(U V)^{2}$ e $U^{3} V=U^{2}(U V)$.

Prova: (i) Para $u_{1}$, uv, $v_{1}$ em N, temos $J\left(u_{1}, u v, v_{1}\right)=u_{1}\left[(u v) v_{1}\right]+$ $+(\mathrm{uv})\left(\mathrm{u}_{1} \mathrm{v}_{1}\right)+\mathrm{v}_{1}\left[\mathrm{u}_{1}(\mathrm{uv})\right]=0$ e como $\mathrm{u}_{1}(\mathrm{uv}) \in \mathrm{U}(\mathrm{UV}) \subseteq \mathrm{U}^{2} \subseteq \mathrm{V}$, segue que $\mathrm{v}_{1}\left[\mathrm{u}_{1}(\mathrm{uv})\right]=0$ e portanto $\mathrm{u}_{1}\left[(\mathrm{uv}) \mathrm{v}_{1}\right]=-(\mathrm{uv})\left(\mathrm{u}_{1} \mathrm{v}_{1}\right)$, o que mostra que todo gerador aditivo de cada um dos subespaços $\mathrm{U}[(\mathrm{UV}) \mathrm{V}]$ e $(\mathrm{UV})^{2}$ é também um gerador aditivo do outro, e assim obtemos a igualdade 
$\mathrm{U}[(\mathrm{UV}) \mathrm{V}]=(\mathrm{UV})^{2}$.

(ii) Analogamente ao caso (i), temos $\mathrm{J}\left(\mathrm{uu}_{1}, \mathrm{u}_{2}, \mathrm{v}\right)=\left(\mathrm{uu}_{1}\right)\left(\mathrm{u}_{2} \mathrm{v}\right)+$ $+\mathrm{v}\left[\left(\mathrm{uu}_{1}\right) \mathrm{u}_{2}\right]+\mathrm{u}_{2}\left[\left(\mathrm{uu}_{1}\right) \mathrm{v}\right]=0$ e como $\mathrm{uu}_{1} \in \mathrm{U}^{2} \subseteq \mathrm{V}$, segue que $\left(\mathrm{uu}_{1}\right) \mathrm{v}=0$ e portanto $\left[\left(\mathrm{uu}_{1}\right) \mathrm{u}_{2}\right] \mathrm{v}=-\left(\mathrm{uu}_{1}\right)\left(\mathrm{u}_{2} \mathrm{v}\right)$, o que mostra a igualdade $\mathrm{U}^{3} \mathrm{~V}=\mathrm{U}^{2}(\mathrm{UV})$, pela mesma razão já explicada acima.

Observação: Decorre daí, por exemplo, que $\left(U^{3} V\right) V=\left[U^{2}(U V)\right] V$. De modo geral, $\left(\mathrm{U}^{3} \mathrm{~V}\right) \mathrm{m}(\mathrm{U}, \mathrm{V})=\left[\mathrm{U}^{2}(\mathrm{UV})\right] \mathrm{m}(\mathrm{U}, \mathrm{V})$. De maneira similar. $\mathrm{U}[(\mathrm{UV}) \mathrm{V}] \mathrm{m}(\mathrm{U}, \mathrm{V})=(\mathrm{UV})^{2} \mathrm{~m}(\mathrm{U}, \mathrm{V})$.

Ainda como parte do Problema 2, provamos o seguinte Lema 3.2. Para todo $k \geq 1$, indicamos por $\mathrm{U}^{k} \mathrm{~V}^{(n)}$ o subespaço $\left[\left(\mathrm{U}^{k} \mathrm{~V}\right) \mathrm{V}\right] \mathrm{V} \ldots \mathrm{V}$ com $n$ fatores V. Analogamente, $\mathrm{U}^{(n)} \mathrm{V}$ indicará o subespaço $\mathrm{U}\{\mathrm{U} \ldots[\mathrm{U}(\mathrm{UV})]\}$, com $n$ fatores U.

Lema 3.2 Consideremos $A=F e \oplus U \oplus V$ uma t-álgebra de posto 3 e $m(U, V)$ um. P-subespaço de A. Então para todo $n \geq 1$, valem as igualdades:

(i) $U^{2 n+1} m(U, V)=U\left[U^{2 n} m(U, V)\right]$, se $m(U, V) \subseteq U$.

(ii) $U^{2 n+1} m(U, V)=U^{2 n}[U m(U, V)]$, se $m(U, V) \subseteq V$.

(iii) $U\left[U^{2 n+1} m(U, V)\right]=U^{2 n+1}[U m(U, V)]=U\left\{U^{2 n}[U m(U, V)]\right\}$, se $m(U, V) \subseteq V$.

(iv) $U\left(U V^{(2 n+1)}\right)=(U V)\left(U V^{(2 n)}\right)$.

Prova: (i) e (ii) Considerando $\mathrm{t} \in \mathrm{U}^{2 n}, \mathrm{u} \in \mathrm{U}$ e $\mathrm{x} \in \mathrm{m}(\mathrm{U}, \mathrm{V})$, segue que (tu) $\mathrm{x} \in\left(\mathrm{U}^{2 n} \mathrm{U}\right) \mathrm{m}(\mathrm{U}, \mathrm{V})=\mathrm{U}^{2 n+1} \mathrm{~m}(\mathrm{U}, \mathrm{V})$. Da identidade de Jacobi, obtemos $(\mathrm{tu}) \mathrm{x}+(\mathrm{tx}) \mathrm{u}+(\mathrm{ux}) \mathrm{t}=0$. No caso em que $\mathrm{m}(\mathrm{U}, \mathrm{V}) \subseteq \mathrm{U}$. $(\mathrm{ux}) \mathrm{t} \in \mathrm{U}^{2} \mathrm{U}^{2 n} \subseteq \mathrm{V}^{2}=0$ e portanto $(\mathrm{tu}) \mathrm{x}=-(\mathrm{tx}) \mathrm{u}$. Como $(\mathrm{tx}) \mathrm{u} \in$ 
$\in\left[\mathrm{U}^{2 n} \mathrm{~m}(\mathrm{U}, \mathrm{V})\right] \mathrm{U}=\mathrm{U}\left[\mathrm{U}^{2 n} \mathrm{~m}(\mathrm{U}, \mathrm{V})\right]$, segue a igualdade (i). Se tivermos $\mathrm{m}(\mathrm{U}, \mathrm{V}) \subseteq \mathrm{V}$, então $(\mathrm{tx}) \mathrm{u} \in \mathrm{V}^{2} \mathrm{U}=0$ e portanto $(\mathrm{tu}) \mathrm{x}=-(\mathrm{ux}) \mathrm{t}$. De (ux) $\mathrm{t} \in[\mathrm{Um}(\mathrm{U}, \mathrm{V})] \mathrm{U}^{2 n}$, segue a igualdade (ii).

(iii) Considerando $\mathrm{u} \in \mathrm{U}, \mathrm{u}_{1} \in \mathrm{U}^{2 n+1} \subseteq \mathrm{U}$ e $\mathrm{v} \in \mathrm{m}(\mathrm{U}, \mathrm{V})$, da identidade de Jacobi temos $u\left(u_{1} v\right)=-u_{1}(u v)$, pois $\left(u_{1}\right) v \in V^{2}=0$. Como $u\left(u_{1} v\right) \in$ $\in \mathrm{U}\left[\mathrm{U}^{2 n+1} \mathrm{~m}(\mathrm{U}, \mathrm{V})\right]$ e $\mathrm{u}_{1}(\mathrm{uv}) \in \mathrm{U}^{2 n+1}[\mathrm{Um}(\mathrm{U}, \mathrm{V})]$, obtemos a igualdade $\mathrm{U}\left[\mathrm{U}^{2 n+1} \mathrm{~m}(\mathrm{U}, \mathrm{V})\right]=\mathrm{U}^{2 n+1}[\mathrm{Um}(\mathrm{U}, \mathrm{V})]$. Tomando agora $\mathrm{t} \in \mathrm{U}^{2 n}, \mathrm{u} \in \mathrm{U}$, $\mathrm{u}_{1} \in \mathrm{Um}(\mathrm{U}, \mathrm{V})$, temos que $(\mathrm{tu}) \mathrm{u}_{1} \in \mathrm{U}^{2 n+1}[\mathrm{Um}(\mathrm{U}, \mathrm{V})]$. Da identidade (1.13), obtemos $(\mathrm{tu}) \mathrm{u}_{1}=-\left(\mathrm{tu}_{1}\right) \mathrm{u}$, pois $\left(\mathrm{uu}_{1}\right) \mathrm{t} \in \mathrm{U}^{2} \mathrm{U}^{2 n} \subseteq \mathrm{V}^{2}=0$. Como $\left(\mathrm{tu}_{1}\right) \mathrm{u} \in\left\{\mathrm{U}^{2 n}[\mathrm{Um}(\mathrm{U}, \mathrm{V})]\right\} \mathrm{U}=\mathrm{U}\left\{\mathrm{U}^{2 n}[\mathrm{Um}(\mathrm{U}, \mathrm{V})]\right\}$, segue a última igualdade de (iii).

(iv) Finalmente, considerando $\mathrm{u} \in \mathrm{U}, \mathrm{u}_{1} \in \mathrm{UV}^{(2 n)}$ e $\mathrm{v} \in \mathrm{V}$, da identidade de Jacobi segue que $\mathrm{u}\left(\mathrm{u}_{1} \mathrm{v}\right)=-\mathrm{u}_{1}(\mathrm{uv})$ e portanto $\mathrm{U}\left(\mathrm{UV}^{(2 n+1)}\right)=$ $=\mathrm{UV}^{(2 n)}$.

Corolário 3.2.1 Nas condições do Lema 3.2, valem as igualdades:

(i) $U^{2 m-1} U^{2 m+1}=U\left[U^{2 m-2} U^{2 m+1}\right]$.

(ii) $U^{2 n+1} U^{2 m+1}=U\left[U^{2 n} U^{2 m+1}\right]$.

(iii) $U^{2 n+1} U^{2 m}=U^{2 n} U^{2 m+1}$.

Prova: (i) Considerando $n=m-1$ (e portanto $2 n+1=2 m-1$ ) e $\mathrm{m}(\mathrm{U}, \mathrm{V})=\mathrm{U}^{2 m+1}$ em (i) do Lema 3.2 , obteremos $\mathrm{U}^{2 m-1} \mathrm{U}^{2 m+1}=$ $=\mathrm{U}\left[\mathrm{U}^{2 m-2} \mathrm{U}^{2 m+1}\right]$.

(ii) Basta considerarmos no Lema 3.2 , (i), $m(U, V)=U^{2 m+1}$ e teremos a igualdade dada em (ii).

(iii) Novamente no Lema 3.2, (ii), considerando $\mathrm{m}(\mathrm{U}, \mathrm{V})=\mathrm{U}^{2 m}$, obtemos $\mathrm{U}^{2 n+1} \mathrm{U}^{2 m}=\mathrm{U}^{2 n}\left[\mathrm{UU}^{2 m}\right]=\mathrm{U}^{2 n} \mathrm{U}^{2 m+1}$. 
Lema 3.3 Se os P-monômios $m_{1}, m_{2}$ e $m_{3}$ não estão simultaneamente contidos em. $U$, então ocorre uma das seguintes situaçôes:

(i) $m_{1}\left(m_{2} m_{3}\right)=m_{2}\left(m_{1} m_{3}\right)=m_{3}\left(m_{1} m_{2}\right)=0$.

(ii) um dos três produtos de P-monômios é nulo e os outros dois produtos coincidem.

Prova: Sejam dados três P-monômios, não todos contidos em U. Então existem três possibilidades a considerar. Na primeira, todos estão contidos em V e portanto, de (1.10), segue que $\mathrm{m}_{1}\left(\mathrm{~m}_{2} \mathrm{~m}_{3}\right)=0$. Analogamente, $\left(\mathrm{m}_{1} \mathrm{~m}_{2}\right) \mathrm{m}_{3}=\mathrm{m}_{2}\left(\mathrm{~m}_{1} \mathrm{~m}_{3}\right)=0$. Na segunda, temos por exemplo $\mathrm{m}_{1} \subseteq \mathrm{U}$ e $\mathrm{m}_{2}, \mathrm{~m}_{3}$ contidos em V. Neste caso, ainda por $(1.10), \mathrm{m}_{1}\left(\mathrm{~m}_{2} \mathrm{~m}_{3}\right)=0$ e $\mathrm{m}_{2}\left(\mathrm{~m}_{1} \mathrm{~m}_{3}\right)=\left(\mathrm{m}_{2} \mathrm{~m}_{1}\right) \mathrm{m}_{3}$. Por último, pode ocorrer dois $\mathrm{P}$-monômios contidos em U e o outro em V (por exemplo, $\mathrm{m}_{3}$ ). Neste caso, (novamente por $(1.10)),\left(\mathrm{m}_{1} \mathrm{~m}_{2}\right) \mathrm{m}_{3}=0$ e $\left(\mathrm{m}_{2} \mathrm{~m}_{3}\right) \mathrm{m}_{1}=\mathrm{m}_{2}\left(\mathrm{~m}_{3} \mathrm{~m}_{1}\right)$.

Corolário 3.3.1 Nas condições do Lema 3.3, se $m_{1}=U^{2 n+1}, m_{2} \subseteq$ $\subseteq U$ e $m_{3} \subseteq V$, então

$$
U^{2 n+1}\left[m_{2} m_{3}\right]=m_{2}\left[U^{2 n+1} m_{3}\right]
$$

Prova: Basta ver que $\mathrm{m}_{1}, \mathrm{~m}_{2}, \mathrm{~m}_{3}$ nas hipóteses acima satisfazem a terceira possibilidade descrita na prova do Lema 3.3.

Como casos particulares do Corolário 3.3.1, obtemos os dois seguintes resultados:

Corolário 3.3.2 $\left(U^{2 n+1}\right)^{2}=U\left(U^{2 n} U^{2 n+1}\right)$, para todo $n \geq 1$.

Prova: Tomar $\mathrm{m}_{2}=\mathrm{U}$ e $\mathrm{m}_{3}=\mathrm{U}^{2 n}$ no Corolário 3.3.1. 
Corolário 3.3.3 $\left(U^{2 n} U^{2 m+1}\right) V=U^{2 n}\left(U^{2 m+1} V\right)$, para todos $n, m \geq 1$.

Prova: Basta ver que $\mathrm{m}_{1}=\mathrm{U}^{2 m+1}, \mathrm{~m}_{2}=\mathrm{U}^{2 n}$ e $\mathrm{m}_{3}=\mathrm{V}$ satisfazem a segunda possibilidade descrita no Lema 3.3 e portanto $\mathrm{m}_{2}\left(\mathrm{~m}_{1} \mathrm{~m}_{3}\right)=$ $=\left(\mathrm{m}_{2} \mathrm{~m}_{1}\right) \mathrm{m}_{3}$. Ou seja, $\mathrm{U}^{2 n}\left(\mathrm{U}^{2 m+1} \mathrm{~V}\right)=\left(\mathrm{U}^{2 n} \mathrm{U}^{2 m+1}\right) \mathrm{V}$.

Lema 3.4 Sejam $m_{1}(U, V)$ e $m_{2}(U, V)$ subespaços monomiais de uma t-álgebra $A=F e \oplus U \oplus V$ de posto 3, ambos contidos em $V$. Então vale a igualdade $\left[U^{2 n+1} m_{1}\right] m_{2}=U^{2 n}\left\{\left[U m_{1}\right] m_{2}\right\}$.

Prova: Considerando $\mathrm{t} \in \mathrm{U}^{2 n}, \mathrm{u} \in \mathrm{U}, \mathrm{v}_{1} \in \mathrm{m}_{1}$ e $\mathrm{v}_{2} \in \mathrm{m}_{2}$, teremos $\left[(\mathrm{tu}) \mathrm{v}_{1}\right] \mathrm{v}_{2} \in\left[\mathrm{U}^{2 n+1} \mathrm{~m}_{1}\right] \mathrm{m}_{2}$. Mas de $\mathrm{J}\left(\mathrm{t}, \mathrm{u}, \mathrm{v}_{1}\right)=0$, obtemos $(\mathrm{tu}) \mathrm{v}_{1}=$ $=-\mathrm{t}\left(\mathrm{uv}_{1}\right)$, pois $\mathrm{u}\left(\mathrm{tv}_{1}\right) \in \mathrm{U}\left[\mathrm{U}^{2 n} \mathrm{~m}_{1}\right] \subseteq \mathrm{UV}^{2}=0$. Portanto, $\left[(\mathrm{tu}) \mathrm{v}_{1}\right] \mathrm{v}_{2}=$ $=-\left[(t u) v_{1}\right] v_{2}$. Novamente usando-se a identidade de Jacobi, agora para os elementos $t, u_{1}, v_{2}$, obtemos $\left[t\left(u v_{1}\right)\right] v_{2}=-t\left[\left(u v_{1}\right) v_{2}\right]$, pois $\left(u_{1}\right)\left(t_{2}\right) \in$ $\in(\mathrm{UV})\left[\mathrm{U}^{2 n} \mathrm{~m}_{2}\right] \subseteq(\mathrm{UV}) \mathrm{V}^{2}=0$. Comot $\left[\left(\mathrm{uv}_{1}\right) \mathrm{v}_{2}\right] \in \mathrm{U}^{2 n}\left\{\left[\mathrm{Um}_{1}\right] \mathrm{m}_{2}\right\}$, segue o resultado.

Corolário 3.4.1 $\quad U^{2 n+1} V^{(2)}=U^{2 n}\left[U V^{(2)}\right]$, para todo $n \geq 1$.

Prova: Tomar $\mathrm{m}_{1}=\mathrm{m}_{2}=\mathrm{V}$ no Lema 3.4 .

Lema 3.5 Se $A=F e \oplus U \oplus V$ é uma t-álgebra de posto 3, então temos a seguinte igualdade de subespaços monomiais, para todo $n \geq 1$

$$
U^{3} V^{(n)}=U^{2}\left[U V^{(n)}\right]
$$

Prova: A prova será feita por indução sobre n. Do Lema 3.1, temos que $\mathrm{U}^{3} \mathrm{~V}=\mathrm{U}^{2}(\mathrm{UV})$, o que mostra que a tese é verdadeira para $n=1$. Suponhamos que a igualdade acima valha para $n$ - 1 fatores $V$. Consideremos então $\mathrm{t} \in \mathrm{U}^{2}, \mathrm{v} \in \mathrm{V}$ e $\mathrm{u} \in \mathrm{UV}^{(n-1)}$. Usando-se a identidade (1.13) para t. $\mathrm{v}$ e $\mathrm{u}$, obteremos $(\mathrm{tu}) \mathrm{v}=-\mathrm{t}(\mathrm{uv})$, pois $\mathrm{u}(\mathrm{tv}) \in \mathrm{U}\left[\mathrm{U}^{2} \mathrm{~V}\right]=0$ e como $(\mathrm{tu}) \mathrm{v} \in$ 
$\in\left[\mathrm{U}^{2}\left(\mathrm{UV}^{(n-1)}\right)\right] \mathrm{V}$, pela hipótese de indução, $(\mathrm{tu}) \mathrm{v} \in\left[\mathrm{U}^{3} \mathrm{~V}^{(n-1)}\right] \mathrm{V}=\mathrm{U}^{3} \mathrm{~V}^{(n)}$. Por outro lado, $\mathrm{t}(\mathrm{uv}) \in \mathrm{U}^{2}\left[\left(\mathrm{UV}^{(n-1)}\right) \mathrm{V}\right]=\mathrm{U}^{2}\left(\mathrm{UV}^{(n)}\right)$, o que completa a prova.

Lema 3.6 Se $A=F e \oplus U \oplus V$ é uma t-álgebra de posto 3, então, para todo $n \geq 1$, temos a seguinte igualdade de P-monômios:

$$
U^{2}\left(U^{(2 n+1)} V\right)=U^{3}\left(U^{(2 n)} V\right) .
$$

Prova: Consideremos os elementos $\mathrm{v} \in \mathrm{U}^{2}, \mathrm{u} \in \mathrm{U}$ e $\mathrm{t} \in \mathrm{U}^{(2 n)} \mathrm{V}$. Note que $\mathrm{U}^{(2 n)} \mathrm{V} \subseteq \mathrm{V}$, pelo Corolário 1.1.1. Aplicando-se a $\mathrm{v}, \mathrm{u}, \mathrm{t}$ a identidade de Jacobi, obtemos v(ut) $=-\mathrm{t}(\mathrm{uv})$, uma vez que vt $\in \mathrm{U}^{2} \mathrm{~V} \subseteq \mathrm{V}^{2}=0$. Como $\mathrm{v}(\mathrm{ut}) \in \mathrm{U}^{2}\left[\mathrm{U}\left(\mathrm{U}^{(2 n)} \mathrm{V}\right)\right]=\mathrm{U}^{2}\left[\mathrm{U}^{(2 n+1)} \mathrm{V}\right]$ e $\mathrm{t}(\mathrm{uv}) \in\left(\mathrm{U}^{(2 n)} \mathrm{V}\right) \mathrm{U}^{3}=\mathrm{U}^{3}\left(\mathrm{U}^{(2 n)} \mathrm{V}\right)$, segue o resultado.

Lema 3.7 Se $A=F e \oplus U \oplus V$ é uma t-álgebra de posto 3, então, para todo $n \geq 1$, temos a seguinte igualdade de P-subespaços:

$$
(U V)\left(U^{2 n+1} V\right)=U\left[U^{2 n+1} V^{(2)}\right]=U^{2 n+1}\left(U V^{(2)}\right) .
$$

Prova: Usando a identidade (1.13) para os elementos $\mathrm{u} \in \mathrm{U}, \mathrm{v} \in \mathrm{V}$ e $\mathrm{u}_{1} \in \mathrm{U}^{2 n+1} \mathrm{~V}$. Assim, $(\mathrm{uv}) \mathrm{u}_{1}=-\left(\mathrm{u}_{1} \mathrm{v}\right) \mathrm{u}$, pois $\left(\mathrm{uu}_{1}\right) \mathrm{v} \in \mathrm{U}^{2 n} \mathrm{~V}=0$ e como $(u v) \mathrm{u}_{1} \in(U V)\left(U^{2 n+1} \mathrm{~V}\right)$ e $\left(\mathrm{u}_{1} \mathrm{v}\right) \mathrm{u}=-\mathrm{u}\left(\mathrm{u}_{1} \mathrm{v}\right) \in \mathrm{U}\left(\mathrm{U}^{2 n+1} \mathrm{~V}^{(2)}\right)$, segue a primeira igualdade. Considerando agora os elementos $u \in U V, u_{1} \in$ $\in \mathrm{U}^{2 n+1}$ e $\mathrm{v} \in \mathrm{V}$, de $\mathrm{J}\left(\mathrm{u}, \mathrm{u}_{1}, \mathrm{v}\right)=0$ obtemos $\mathrm{u}\left(\mathrm{u}_{1} \mathrm{v}\right)=-\mathrm{u}_{1}(\mathrm{uv})$, uma vez que $\left(\mathrm{uu}_{1}\right) \mathrm{v} \in \mathrm{U}^{2 n} \mathrm{~V}=0$. Mas $\mathrm{u}\left(\mathrm{u}_{1} \mathrm{v}\right) \in(\mathrm{UV})\left(\mathrm{U}^{2 n+1} \mathrm{~V}\right)$ e $\mathrm{u}_{1}(\mathrm{uv}) \epsilon$ $\in \mathrm{U}^{2 n+1}\left(\mathrm{UV}^{(2)}\right)$ e, portanto, segue a segunda igualdade.

Observação final: Todos os resultados deste capítulo podem ser entendidos como uma forma fraca de associatividade em $\mathrm{A}$, isto é, vale associatividade para certos produtos de subespaços. 


\section{Capítulo 4}

\section{Sobre a dimensão de $\mathbf{A}$, baseada em P-monômios}

Neste capítulo faremos estimativas sobre a dimensão de uma t-álgebra de posto 3, sabendo-se que um dado P-monômio é não nulo. Em cada caso, exibimos um exemplo minimal que comprova a estimativa feita.

\subsection{Lemas auxiliares}

Consideremos $\mathrm{A}=\mathrm{Fe} \oplus \mathrm{U} \oplus \mathrm{V}$ uma t-álgebra de posto 3 , de tipo $(1+r, s)$. A fim de tornarmos a leitura mais agradável, reuniremos em dois Lemas preliminares todas as identidades que nos serão úteis neste capítulo. Tais identidades são obtidas, de um modo geral, a partir da identidade de Jacobi aplicada, sucessivamente, a convenientes elementos de $\mathrm{N}=$ ker $\omega$.

Lema 4.1 Seja $A=F e \oplus U \oplus V$ uma t-álgebra de posto 3. Para todos $u, u_{1}, u_{2}, u_{3} \in U$ e $v, v_{1}, v_{2}, v_{3} \in V$, valem as identidades:

$$
\begin{gathered}
2 u\left(u u_{1}\right)=-u^{2} u_{1} \\
2\left(u_{1} u_{2}\right)\left(u_{1} v_{1}\right)=\left(u_{1}^{2} u_{2}\right) v_{1}=-u_{1}^{2}\left(u_{2} v_{1}\right) \\
(u v) v_{1}=-\left(u v_{1}\right) v
\end{gathered}
$$




$$
\begin{gathered}
u\left(u_{1} v\right)=-u_{1}\left(u v^{\prime}\right) \\
\left(u_{1} v_{1}\right)\left(u_{2} v_{2}\right)=-u_{1}\left[\left(u_{2} v_{2}\right) v_{1}\right]=-u_{2}\left[\left(u_{1} v_{1}\right) v_{2}\right] \\
u_{1}\left[\left(u_{1}^{2} u_{2}\right) u_{3}\right]=-\left(u_{1}^{2} u_{2}\right)\left(u_{1} u_{3}\right)=u_{1}^{2}\left[\left(u_{1} u_{3}\right) u_{2}\right]=-u_{1}^{2}\left[\left(u_{1} u_{2}\right) u_{3}\right] \\
u_{2}\left\{u_{1}^{2}\left[\left(u_{2} v_{2}\right) v_{1}\right]\right\}=-\left(u_{1}^{2} u_{2}\right)\left[\left(u_{2} v_{2}\right) v_{1}\right] \\
2 u_{1}\left[\left(u_{1} v_{1}\right)\left(u_{2} v_{2}\right)\right]=-2 u_{1}\left\{u_{1}\left[\left(u_{2} v_{2}\right) v_{1}\right]\right\}=u_{1}^{2}\left[\left(u_{2} v_{2}\right) v_{1}\right]
\end{gathered}
$$

Prova: (4.1) Basta ver que $0=J\left(u, u, u_{1}\right)=2 u\left(u_{1}\right)+u^{2} u_{1}$.

(4.2) De $0=2 J\left(\mathrm{u}_{1} \mathrm{u}_{2}, \mathrm{u}_{1}, \mathrm{v}_{1}\right)$ segue que $\mathrm{y}=2\left(\mathrm{u}_{1} \mathrm{u}_{2}\right)\left(\mathrm{u}_{1} \mathrm{v}_{1}\right)=-2\left[\mathrm{u}_{1}\left(\mathrm{u}_{1} \mathrm{u}_{2}\right)\right] \mathrm{v}_{1}$ (pois $\left(\mathrm{u}_{1} \mathrm{u}_{2}\right) \mathrm{v}_{1} \in \mathrm{V}^{2}=0$ ) e por (4.1) temos que $\mathrm{y}=\left(\mathrm{u}_{1}^{2} \mathrm{u}_{2}\right) \mathrm{v}_{1}$. Usandose agora a identidade 1.13 para os elementos $\mathrm{u}_{1}^{2}, \mathrm{u}_{2}, \mathrm{v}_{1}$, segue a última igualdade de (4.2) .

(4.3) Basta ver que $0=J\left(u, v, v_{1}\right)=(u v) v_{1}+\left(u_{1}\right) v$.

(4.4) Basta ver que $0=J\left(u, u_{1}, v\right)=u\left(u_{1} v\right)+u_{1}(u v)$.

(4.5) Aplicando-se a identidade de Jacobi aos elementos $\mathrm{u}_{1}, \mathrm{v}_{1}, \mathrm{u}_{2} \mathrm{v}_{2}$ e depois a $\mathrm{u}_{2}, \mathrm{v}_{2}, \mathrm{u}_{1} \mathrm{v}_{1}$ obtemos, respectivamente, $\left(\mathrm{u}_{1} \mathrm{v}_{1}\right)\left(\mathrm{u}_{2} \mathrm{v}_{2}\right)=-\mathrm{u}_{1}\left[\left(\mathrm{u}_{2} \mathrm{v}_{2}\right) \mathrm{v}_{1}\right]$ $\mathrm{e}\left(\mathrm{u}_{1} \mathrm{v}_{1}\right)\left(\mathrm{u}_{2} \mathrm{v}_{2}\right)=-\mathrm{u}_{2}\left[\left(\mathrm{u}_{1} \mathrm{v}_{1}\right) \mathrm{v}_{2}\right]$.

(4.6) Aplicando-se a identidade (1.13) aos elementos $u_{1}, u_{1}^{2} u_{2}, u_{3}$ e de (1.18) segue que $\mathrm{u}_{1}\left[\left(\mathrm{u}_{1}^{2} \mathrm{u}_{2}\right) \mathrm{u}_{3}\right]=-\left(\mathrm{u}_{1}^{2} \mathrm{u}_{2}\right)\left(\mathrm{u}_{1} \mathrm{u}_{3}\right)=\mathrm{u}_{1}^{2}\left[\mathrm{u}_{2}\left(\mathrm{u}_{1} \mathrm{u}_{3}\right)\right]$, sendo esta última igualdade consequência de $\mathrm{J}\left(\mathrm{u}_{1}^{2}, \mathrm{u}_{2}, \mathrm{u}_{1} \mathrm{u}_{3}\right)=0$ e de $\mathrm{U}^{2} \mathrm{U}^{2} \subseteq \mathrm{V}^{2}=$ $=0$. Finalmente, como consequência de $\mathrm{J}\left(\mathrm{u}_{1}, \mathrm{u}_{2}, \mathrm{u}_{3}\right)=0$ e de (1.16), segue que $u_{1}^{2}\left[u_{2}\left(u_{1} u_{3}\right)\right]=-u_{1}^{2}\left[\left(u_{1} u_{2}\right) u_{3}\right]$.

(4.7) Da identidade de Jacobi aplicada aos elementos $u_{2}, u_{1}^{2},\left(u_{2} v_{2}\right) v_{1}$ segue que $\mathrm{u}_{2}\left\{\mathrm{u}_{1}^{2}\left[\left(\mathrm{u}_{2} \mathrm{v}_{2}\right) \mathrm{v}_{1}\right]\right\}=-\left(\mathrm{u}_{1}^{2} \mathrm{u}_{2}\right)\left[\left(\mathrm{u}_{2} \mathrm{v}_{2}\right) \mathrm{v}_{1}\right]$, pois $\mathrm{U}^{2}\{\mathrm{U}[(\mathrm{UV}) \mathrm{V}]\} \subseteq$ $\subseteq \mathrm{U}^{2} \mathrm{U}^{2} \subseteq \mathrm{V}^{2}=0$.

(4.8) Temos que $2 \mathrm{u}_{1}\left[\left(\mathrm{u}_{1} \mathrm{v}_{1}\right)\left(\mathrm{u}_{2} \mathrm{v}_{2}\right)\right]=-2\left\{\mathrm{u}_{1}\left[\mathrm{u}_{1}\left[\left(\mathrm{u}_{2} \mathrm{v}_{2}\right) \mathrm{v}_{1}\right]\right\}=\mathrm{u}_{1}^{2}\left[\left(\mathrm{u}_{2} \mathrm{v}_{2}\right) \mathrm{v}_{1}\right]\right.$. sendo as duas igualdades consequências de (4.5) e de (4.1), respectivamente. 
Lema 4.2 Seja $A=F e \oplus U \oplus V$ uma t-álgebra de posto 3. Para todos $u, u_{1}, u_{2}, u_{3} \in U$ e v, $v_{1}, v_{2}, v_{3} \in V$. valem as identidades:

$$
\begin{gathered}
(u v)\left(u v_{1}\right)=0 \\
\left(u_{1} v\right)\left(u_{2} v\right)=0 \\
u\left[\left(u v_{1}\right) v_{1}\right]=u\left[u_{1}^{2}(u v)\right]=0 \\
u_{i}\left[\left(u_{1} u_{2}\right)\left(u_{1}^{2} u_{3}\right)\right]=u_{i}\left\{u_{1}^{2}\left[u_{3}\left(u_{1} u_{2}\right)\right]\right\}=u_{i}\left[\left(u_{1} u_{3}\right)\left(u_{1}^{2} u_{2}\right)\right]=0 \\
u_{3}\left\{u_{2}\left[u_{3}\left(u_{1}^{2} u_{2}\right)\right]\right\}=0 \\
\left(u_{1} v_{2}\right)\left[\left(u_{2} v_{2}\right) v_{1}\right]=0 \\
\left\{u_{1}\left[\left(u_{1} v_{1}\right)\left(u_{2} v_{2}\right)\right]\right\}\left(u_{2} v_{2}\right)=0 \\
\left(u_{1} v_{1}\right)\left[\left(u_{1} v_{1}\right)\left(u_{2} v_{2}\right)\right]=0 \\
u_{1}^{2}\left[\left(u_{1} v_{1}\right) v_{2}\right]=0 \\
u_{1}\left\{\left[u_{1}^{2}\left(u_{2} v_{1}\right)\right]\left(u_{2} v_{2}\right)\right\}=0 \\
u_{1}\left\{\left(u_{2} u_{1}^{2}\right)\left[\left(u_{2} v_{2}\right) v_{1}\right]\right\}=0 \\
u_{1}^{2}\left[\left(u_{1} u_{2}\right)\left(u_{2} v_{2}\right)\right]=0 \\
u_{1}^{2}\left\{\left[u_{2}\left(u_{1} v_{1}\right)\right]\left(u_{2} v_{2}\right)\right\}=0 \\
u_{2}\left[\left(u_{1} u_{2}\right)\left(u_{1} v_{1}\right)\right]=0 \\
u_{2}\left\{u_{1}\left\{\left[u_{2}\left(u_{1} v_{1}\right)\right]\left(u_{2} v_{2}\right)\right\}\right\}=0
\end{gathered}
$$

Prova: (4.9) De (1.17) temos $0=\left[\mathrm{u}\left(\mathrm{v}+\mathrm{v}_{1}\right)\right]^{2}=\left(\mathrm{uv}+\mathrm{uv}_{1}\right)^{2}=(\mathrm{uv})^{2}+$ $+\left(u_{1}\right)^{2}+2(u v)\left(u v_{1}\right)=2(u v)\left(u_{1}\right)$ e portanto $(u v)\left(u_{1}\right)=0$.

(4.10) Ainda de (1.17) temos $0=\left[\left(\mathrm{u}_{1}+\mathrm{u}_{2}\right) \mathrm{v}\right]^{2}=\left(\mathrm{u}_{1} \mathrm{v}+\mathrm{u}_{2} \mathrm{v}\right)^{2}=\left(\mathrm{u}_{1} \mathrm{v}\right)^{2}+$ $+\left(\mathrm{u}_{2} \mathrm{v}\right)^{2}+2\left(\mathrm{u}_{1} \mathrm{v}\right)\left(\mathrm{u}_{2} \mathrm{v}\right)=2\left(\mathrm{u}_{1} \mathrm{v}\right)\left(\mathrm{u}_{2} \mathrm{v}\right)$.

(4.11) Usando-se sucessivamente as identidades (1.13) para os elementos $\mathrm{u}, \mathrm{v}, \mathrm{uv}_{\mathrm{l}},(1.14)$ e (4.9), segue que $\mathrm{u}\left[\left(\mathrm{uv}_{1}\right) \mathrm{v}\right]=0$ e portanto, de (4.3) segue que também $u\left[(u v) v_{1}\right]=0$. Em particular, para $v_{1}=u_{1}^{2}$, obtemos $\mathrm{u}\left[\mathrm{u}_{1}^{2}(\mathrm{uv})\right]=0$. 
(4.12) Seja $\mathrm{y}=\mathrm{u}_{i}\left[\left(\mathrm{u}_{1} \mathrm{u}_{2}\right)\left(\mathrm{u}_{1}^{2} \mathrm{u}_{3}\right)\right]$. Como $\mathrm{u}_{1} \mathrm{u}_{2}, \mathrm{u}_{1}^{2} \in \mathrm{V}$, de (4.3) segue que $\mathrm{y}=-\mathrm{u}_{i}\left\{\left[\mathrm{u}_{3}\left(\mathrm{u}_{1} \mathrm{u}_{2}\right)\right] \mathrm{u}_{1}^{2}\right\}$. De $\mathrm{J}\left(\mathrm{u}_{1}, \mathrm{u}_{2}, \mathrm{u}_{3}\right)=0$ e de (1.16) obtemos $\mathrm{y}=\mathrm{u}_{i}\left\{\left[\mathrm{u}_{1}\left(\mathrm{u}_{2} \mathrm{u}_{3}\right)+\mathrm{u}_{2}\left(\mathrm{u}_{1} \mathrm{u}_{3}\right)\right] \mathrm{u}_{1}^{2}\right\}=\mathrm{u}_{i}\left\{\mathrm{u}_{1}^{2}\left[\mathrm{u}_{1}\left(\mathrm{u}_{2} \mathrm{u}_{3}\right)\right]\right\}+\mathrm{u}_{i}\left\{\mathrm{u}_{1}^{2}\left[\mathrm{u}_{2}\left(\mathrm{u}_{1} \mathrm{u}_{3}\right)\right]\right\}=$ $=\mathrm{u}_{i}\left\{\mathrm{u}_{1}^{2}\left[\mathrm{u}_{2}\left(\mathrm{u}_{1} \mathrm{u}_{3}\right)\right]\right\}=-\mathrm{u}_{i}\left[\left(\mathrm{u}_{2} \mathrm{u}_{1}^{2}\right)\left(\mathrm{u}_{1} \mathrm{u}_{3}\right)\right]$, sendo que esta última igualdade é aplicação de (4.3). Note que se $i=1$, então $\mathrm{y}=\mathrm{u}_{1}\left\{\mathrm{u}_{1}^{2}\left[\mathrm{u}_{2}\left(\mathrm{u}_{1} \mathrm{u}_{3}\right)\right]\right\}=$ $=0$ por (1.18). Se $i=2$, então $\mathrm{y}=\mathrm{u}_{2}\left\{\mathrm{u}_{1}^{2}\left[\mathrm{u}_{2}\left(\mathrm{u}_{1} \mathrm{u}_{3}\right)\right]\right\}=-\left(\mathrm{u}_{2} \mathrm{u}_{1}^{2}\right)\left[\mathrm{u}_{2}\left(\mathrm{u}_{1} \mathrm{u}_{3}\right)\right]$ (de (4.4)) e portanto y $=0$, por (4.9). Finalmente, se $i=3$, temos que $\mathrm{y}=$ $=-u_{3}\left[\left(u_{2} u_{1}^{2}\right)\left(u_{1} u_{3}\right)\right]$ e da identidade de Jacobi aplicada a $u_{1}, u_{3}, u_{1}^{2} u_{2}$, segue que $2 \mathrm{y}=2 \mathrm{u}_{3}\left\{\mathrm{u}_{1}\left[\mathrm{u}_{3}\left(\mathrm{u}_{1}^{2} \mathrm{u}_{2}\right)\right]+\mathrm{u}_{3}\left[\mathrm{u}_{1}\left(\mathrm{u}_{1}^{2} \mathrm{u}_{2}\right)\right]\right\}=2 \mathrm{u}_{3}\left\{\mathrm{u}_{1}\left[\mathrm{u}_{3}\left(\mathrm{u}_{1}^{2} \mathrm{u}_{2}\right)\right]\right\}$, sendo esta última igualdade consequência de (1.18). Portanto, da aplicação sucessiva de (4.4), (4.1), (4.3) e (1.18) segue que $2 \mathrm{y}=-2 \mathrm{u}_{1}\left\{\mathrm{u}_{3}\left[\mathrm{u}_{3}\left(\mathrm{u}_{1}^{2} \mathrm{u}_{2}\right)\right]\right\}=$ $=\mathrm{u}_{1}\left[\mathrm{u}_{3}^{2}\left(\mathrm{u}_{1}^{2} \mathrm{u}_{2}\right)\right]=-\mathrm{u}_{1}\left[\mathrm{u}_{1}^{2}\left(\mathrm{u}_{2} \mathrm{u}_{3}^{2}\right)\right]=0$.

(4.13) De $J\left(\mathrm{u}_{2}, \mathrm{u}_{3}, \mathrm{u}_{1}^{2} \mathrm{u}_{2}\right)=0$, obtemos $\mathrm{y}=\mathrm{u}_{3}\left\{\mathrm{u}_{2}\left[\mathrm{u}_{3}\left(\mathrm{u}_{1}^{2} \mathrm{u}_{2}\right)\right]\right\}=$ $=-\mathrm{u}_{3}\left\{\mathrm{u}_{3}\left[\mathrm{u}_{2}\left(\mathrm{u}_{1}^{2} \mathrm{u}_{2}\right)\right]\right\}-\mathrm{u}_{3}\left[\left(\mathrm{u}_{2} \mathrm{u}_{3}\right)\left(\mathrm{u}_{2} \mathrm{u}_{1}^{2}\right)\right]=-\mathrm{u}_{3}\left[\left(\mathrm{u}_{2} \mathrm{u}_{3}\right)\left(\mathrm{u}_{2} \mathrm{u}_{1}^{2}\right)\right]$, por (1.14). Da identidade de Jacobi aplicada agora aos elementos $u_{3}, u_{2} u_{3}, u_{2} u_{1}^{2}$ e de $\mathrm{U}^{2} \mathrm{U}^{4}=0$, segue que $2 \mathrm{y}=2\left(\mathrm{u}_{2} \mathrm{u}_{1}^{2}\right)\left[\mathrm{u}_{3}\left(\mathrm{u}_{2} \mathrm{u}_{3}\right)\right]=-\left(\mathrm{u}_{2} \mathrm{u}_{1}^{2}\right)\left(\mathrm{u}_{3}^{2} \mathrm{u}_{2}\right)=0$, sendo estas duas últimas igualdades consequências de (4.1) e (4.9), respectivamente.

(4.14) Basta aplicar (4.3) (para $\left.\left(\mathrm{u}_{2} \mathrm{v}_{2}\right) \mathrm{v}_{1}\right)$ e a seguir (4.10).

(4.15) Da identidade $J\left(u_{1}, u_{1} v_{1}, u_{2} v_{2}\right)=0$ segue que $u_{1}\left[\left(u_{1} v_{1}\right)\left(u_{2} v_{2}\right)\right]=$ $=-\left(\mathrm{u}_{1} \mathrm{v}_{1}\right)\left[\mathrm{u}_{1}\left(\mathrm{u}_{2} \mathrm{v}_{2}\right)\right]-\left(\mathrm{u}_{2} \mathrm{v}_{2}\right)\left[\mathrm{u}_{1}\left(\mathrm{u}_{1} \mathrm{v}_{1}\right)\right]=-\left(\mathrm{u}_{1} \mathrm{v}_{1}\right)\left[\mathrm{u}_{1}\left(\mathrm{u}_{2} \mathrm{v}_{2}\right)\right]$, por $(1.14)$ e dessa forma, obtemos $\left\{\mathrm{u}_{1}\left[\left(\mathrm{u}_{1} \mathrm{v}_{1}\right)\left(\mathrm{u}_{2} \mathrm{v}_{2}\right)\right]\right\}\left(\mathrm{u}_{2} \mathrm{v}_{2}\right)=-\left\{\left(\mathrm{u}_{1} \mathrm{v}_{1}\right)\left[\mathrm{u}_{1}\left(\mathrm{u}_{2} \mathrm{v}_{2}\right)\right]\right\}\left(\mathrm{u}_{2} \mathrm{v}_{2}\right)=$ $=\left\{\left(\mathrm{u}_{2} \mathrm{v}_{2}\right)\left[\mathrm{u}_{1}\left(\mathrm{u}_{2} \mathrm{v}_{2}\right)\right]\right\}\left(\mathrm{u}_{1} \mathrm{v}_{1}\right)$ (por (4.4)) e este último elemento é nulo, pois de (1.13) aplicada a $\mathrm{u}_{2} \mathrm{v}_{2}, \mathrm{u}_{1}, \mathrm{u}_{2} \mathrm{v}_{2}$ e (1.17) segue que $2\left(\mathrm{u}_{2} \mathrm{v}_{2}\right)\left[\mathrm{u}_{1}\left(\mathrm{u}_{2} \mathrm{v}_{2}\right)\right]=$ $=-\left(\mathrm{u}_{2} \mathrm{v}_{2}\right)^{2} \mathrm{u}_{1}=0$.

(4.16) Basta aplicar a identidade de Jacobi aos elementos $\mathrm{u}_{1} \mathrm{v}_{1}, \mathrm{u}_{1} \mathrm{v}_{1}, \mathrm{u}_{2} \mathrm{v}_{2}$ e usar (1.17) para obtermos a identidade (4.16).

(4.17) Das identidades (4.3) e (1.16) temos $\mathrm{u}_{1}^{2}\left[\left(\mathrm{u}_{1} \mathrm{v}_{1}\right) \mathrm{v}_{2}\right]=\left[\left(\mathrm{u}_{1} \mathrm{v}_{1}\right) \mathrm{u}_{1}^{2}\right] \mathrm{v}_{2}=$ $=0$. 
(4.18) Das identidades (4.3) e (4.4) temos $\mathrm{y}=\mathrm{u}_{1}\left\{\left[\mathrm{u}_{1}^{2}\left(\mathrm{u}_{2} \mathrm{v}_{1}\right)\right]\left(\mathrm{u}_{2} \mathrm{v}_{2}\right)\right\}=$ $=-\mathrm{u}_{1}\left\{\left[\left(\mathrm{u}_{2} \mathrm{u}_{1}^{2}\right) \mathrm{v}_{1}\right]\left(\mathrm{u}_{2} \mathrm{v}_{2}\right)\right\}=\mathrm{u}_{1}\left\{\left[\left(\mathrm{u}_{2} \mathrm{v}_{2}\right) \mathrm{v}_{1}\right]\left(\mathrm{u}_{2} \mathrm{u}_{1}^{2}\right)\right\}$. Assim, da identidade de Jacobi aplicada aos elementos $\mathrm{u}_{1}, \quad\left(\mathrm{u}_{2} \mathrm{v}_{2}\right) \mathrm{v}_{1}, \mathrm{u}_{2} \mathrm{u}_{1}^{2}$, obtemos $\mathrm{y}=$ $=-\left[\left(\mathrm{u}_{2} \mathrm{v}_{2}\right) \mathrm{v}_{1}\right]\left[\mathrm{u}_{1}\left(\mathrm{u}_{1}^{2} \mathrm{u}_{2}\right)\right]-\left(\mathrm{u}_{2} \mathrm{u}_{1}^{2}\right)\left\{\mathrm{u}_{1}\left[\left(\mathrm{u}_{2} \mathrm{v}_{2}\right) \mathrm{v}_{1}\right]\right\}=-\left(\mathrm{u}_{2} \mathrm{u}_{1}^{2}\right)\left\{\mathrm{u}_{1}\left[\left(\mathrm{u}_{2} \mathrm{v}_{2}\right) \mathrm{v}_{1}\right]\right\}$, por (1.18). Dessa forma, de (4.5) e de $J\left(u_{2} u_{1}^{2}, u_{1} v_{1}, u_{2} v_{2}\right)=0$, obtemos $\mathrm{y}=\left(\mathrm{u}_{2} \mathrm{u}_{1}^{2}\right)\left[\left(\mathrm{u}_{1} \mathrm{v}_{1}\right)\left(\mathrm{u}_{2} \mathrm{v}_{2}\right)\right]=-\left(\mathrm{u}_{1} \mathrm{v}_{1}\right)\left[\left(\mathrm{u}_{2} \mathrm{u}_{1}^{2}\right)\left(\mathrm{u}_{2} \mathrm{v}_{2}\right)\right]-\left(\mathrm{u}_{2} \mathrm{v}_{2}\right)\left[\left(\mathrm{u}_{2} \mathrm{u}_{1}^{2}\right)\left(\mathrm{u}_{1} \mathrm{v}_{1}\right)\right]=$ $=-\left(u_{2} v_{2}\right)\left[\left(u_{2} u_{1}^{2}\right)\left(u_{1} v_{1}\right)\right]$, sendo que esta última igualdade é consequência de (4.9). Finalmente, de (4.1) obtemos $\mathrm{y}=2\left(\mathrm{u}_{2} \mathrm{v}_{2}\right)\left\{\left[\mathrm{u}_{1}\left(\mathrm{u}_{1} \mathrm{u}_{2}\right)\right]\left(\mathrm{u}_{1} \mathrm{v}_{1}\right)\right\}$. O resultado segue de (4.9).

(4.19) Basta ver que da identidade (4.4) aplicada aos elementos $u_{2} u_{1}^{2}$, $\mathrm{u}_{2} \mathrm{v}_{2}, \mathrm{v}_{1}$ temos $\left(\mathrm{u}_{2} \mathrm{u}_{1}^{2}\right)\left[\left(\mathrm{u}_{2} \mathrm{v}_{2}\right) \mathrm{v}_{1}\right]=-\left(\mathrm{u}_{2} \mathrm{v}_{2}\right)\left[\left(\mathrm{u}_{2} \mathrm{u}_{1}^{2}\right) \mathrm{v}_{1}\right]=-\left[\left(\mathrm{u}_{2} \mathrm{u}_{1}^{2}\right) \mathrm{v}_{1}\right]\left(\mathrm{u}_{2} \mathrm{v}_{2}\right)=$ $=\left[\left(u_{2} v_{1}\right) u_{1}^{2}\right]\left(u_{2} v_{2}\right)$, sendo esta útima igualdade consequência de (4.3). O resultado segue de (4.18).

(4.20) Como $\mathrm{u}_{1} \mathrm{u}_{2} \in \mathrm{V}$, de (4.3) temos que $\mathrm{y}=2 \mathrm{u}_{1}^{2}\left[\left(\mathrm{u}_{1} \mathrm{u}_{2}\right)\left(\mathrm{u}_{2} \mathrm{v}_{2}\right)\right]=$ $=-2 \mathrm{u}_{1}^{2}\left\{\left[\mathrm{u}_{2}\left(\mathrm{u}_{1} \mathrm{u}_{2}\right)\right] \mathrm{v}_{2}\right\}=\mathrm{u}_{1}^{2}\left[\left(\mathrm{u}_{2}^{2} \mathrm{u}_{1}\right) \mathrm{v}_{2}\right]=-\left[\left(\mathrm{u}_{2}^{2} \mathrm{u}_{1}\right) \mathrm{u}_{1}^{2}\right] \mathrm{v}_{2}$, sendo estas duas últimas igualdades consequência de (4.1) e (4.3), respectivamente. Logo, y $=0$, por $(1.16)$.

(4.21) Seja $\mathrm{y}=\mathrm{u}_{1}^{2}\left\{\left[\mathrm{u}_{2}\left(\mathrm{u}_{1} \mathrm{v}_{1}\right)\right]\left(\mathrm{u}_{2} \mathrm{v}_{2}\right)\right\}$. De $J\left(\mathrm{u}_{2}, \mathrm{u}_{1} \mathrm{v}_{1}, \mathrm{u}_{2} \mathrm{v}_{2}\right)=0$ e de (1.14), obtemos $2\left[\mathrm{u}_{2}\left(\mathrm{u}_{1} \mathrm{v}_{1}\right)\right]\left(\mathrm{u}_{2} \mathrm{v}_{2}\right)=-2 \mathrm{u}_{2}\left[\left(\mathrm{u}_{1} \mathrm{v}_{1}\right)\left(\mathrm{u}_{2} \mathrm{v}_{2}\right)\right]=2 \mathrm{u}_{2}\left\{\mathrm{u}_{2}\left[\left(\mathrm{u}_{1} \mathrm{v}_{1}\right) \mathrm{v}_{2}\right]\right\}=$ $=-u_{2}^{2}\left[\left(u_{1} v_{1}\right) v_{2}\right]$, sendo as duas últimas igualdades consequências de (4.5) e (4.1), respectivamente. Portanto, $2 \mathrm{y}=-\mathrm{u}_{1}^{2}\left\{\mathrm{u}_{2}^{2}\left[\left(\mathrm{u}_{1} \mathrm{v}_{1}\right) \mathrm{v}_{2}\right]\right\}$. Usando-se. agora, a identidade (4.3) por duas vezes consecutivas, obtemos $2 \mathrm{y}=$ $\left.=\mathrm{u}_{2}^{2}\left\{\mathrm{u}_{1}^{2}\left[\left(\mathrm{u}_{1} \mathrm{v}_{1}\right) \mathrm{v}_{2}\right]\right\}=-\mathrm{u}_{2}^{2}\left\{\left[\left(\mathrm{u}_{1} \mathrm{v}_{1}\right) \mathrm{u}_{1}^{2}\right] \mathrm{v}_{2}\right]\right\}=0$, sendo que esta última igualdade segue de (1.16).

(4.22) Das identidades (4.3), (4.1) e (4.4) temos $2 \mathrm{u}_{2}\left[\left(\mathrm{u}_{1} \mathrm{u}_{2}\right)\left(\mathrm{u}_{1} \mathrm{v}_{1}\right)\right]=$ $=-2 \mathrm{u}_{2}\left\{\left[\mathrm{u}_{1}\left(\mathrm{u}_{1} \mathrm{u}_{2}\right)\right] \mathrm{v}_{1}\right\}=\mathrm{u}_{2}\left[\left(\mathrm{u}_{1}^{2} \mathrm{u}_{2}\right) \mathrm{v}_{1}\right]=-\left(\mathrm{u}_{2} \mathrm{u}_{1}^{2}\right)\left(\mathrm{u}_{2} \mathrm{v}_{1}\right)$ e assim o resultado é consequência de (4.9).

(4.23) Sejam $y=u_{2}\left\{u_{1}\left\{\left[u_{2}\left(u_{1} v_{1}\right)\right]\left(u_{2} v_{2}\right)\right\}\right\}$ e $t=2\left[u_{2}\left(u_{1} v_{1}\right)\right]\left(u_{2} v_{2}\right)$. Como em (4.21), $t=-u_{2}^{2}\left[\left(u_{1} v_{1}\right) v_{2}\right]$ e dessa forma obtemos $2 y=u_{2}\left(u_{1} t\right)=$ 
$=-\mathrm{u}_{2}\left\{\mathrm{u}_{1}\left\{\mathrm{u}_{2}^{2}\left[\left(\mathrm{u}_{1} \mathrm{v}_{1}\right) \mathrm{v}_{2}\right]\right\}\right\}=\mathrm{u}_{2}\left\{\left[\left(\mathrm{u}_{1} \mathrm{v}_{1}\right) \mathrm{v}_{2}\right]\left(\mathrm{u}_{1} \mathrm{u}_{2}^{2}\right)\right\}$, por (4.4). Da identidade de Jacobi aplicada aos elementos $\mathrm{u}_{2},\left(\mathrm{u}_{1} \mathrm{v}_{1}\right) \mathrm{v}_{2}, \mathrm{u}_{1} \mathrm{u}_{2}^{2}$ e de (1.18), segue que $2 \mathrm{y}=-\left(\mathrm{u}_{1} \mathrm{u}_{2}^{2}\right)\left\{\mathrm{u}_{2}\left[\left(\mathrm{u}_{1} \mathrm{v}_{1}\right) \mathrm{v}_{2}\right]\right\}$. Finalmente, de (4.5), (4.3) e (4.1), obtemos $4 \mathrm{y}=-2\left(\mathrm{u}_{1} \mathrm{u}_{2}^{2}\right)\left\{\mathrm{u}_{1}\left[\left(\mathrm{u}_{2} \mathrm{v}_{2}\right) \mathrm{v}_{1}\right]\right\}=2\left\{\mathrm{u}_{1}\left\{\mathrm{u}_{1}\left[\left(\mathrm{u}_{2} \mathrm{v}_{2}\right) \mathrm{v}_{1}\right]\right\}\right\} \mathrm{u}_{2}^{2}=-\left\{\mathrm{u}_{1}^{2}\left[\left(\mathrm{u}_{2} \mathrm{v}_{2}\right) \mathrm{v}_{1}\right]\right\} \mathrm{u}_{2}^{2}$ e como em (4.21) segue o resultado.

Antes de expor os resultados deste capítulo, vamos explorar um resultado que aparece mencionado brevemente em [20]. Observemos que para cada $\gamma \in \mathrm{F}$ fixado, $2 \gamma \neq 1$, temos uma classe de t-álgebras de posto 3 satisfazendo a equação $\mathrm{x}^{3}-(1+\gamma) \omega(\mathrm{x}) \mathrm{x}^{2}+\gamma \omega(\mathrm{x})^{2} \mathrm{x}=0$. Se A satisfaz a equação $\mathrm{x}^{3}-\omega(\mathrm{x}) \mathrm{x}^{2}=0$ (isto é, o caso $\gamma=0$ ), é possível passar para outra classe na qual $\gamma \neq 0$ definindo-se sobre o F-espaço vetorial A uma nova multiplicação dada por

$$
\mathrm{x} \star \mathrm{y}=(1-2 \gamma) \mathrm{xy}+\gamma[\mathrm{x} \omega(\mathrm{y})+\mathrm{y} \omega(\mathrm{x})]
$$

com $\gamma \in \mathrm{F}$ distinto de $\frac{1}{2}$. Temos os seguintes resultados sobre esta nova álgebra (denotada por $\mathrm{A}_{1}$ ):

Lema 4.3 Seja $A=F e \oplus U \oplus V$ uma t-álgebra de posto 3 satisfazendo a t-equação $x^{3}-\omega(x) x^{2}=0$. Sejam $\gamma \in F, 2 \gamma \neq 1$ e $A_{1}$ álgebra definida acima. Entấo

(a) $A_{1}$ é bárica, comutativa, com função peso w.

(b) A e $A_{1}$ possuem os mesmos idempotentes.

(c) $A_{1}$ satisfaz a t-equação $x^{3}-(1+\gamma) \omega(x) x^{2}+\gamma \omega(x)^{2} x=0$.

(d) A decomposiçâo de Peirce de $A_{1}$, relativa ao idempotente e, é $A_{1}=$ $=F e \oplus U \oplus V$.

Prova:(a) É claro que $\mathrm{A}_{1}$ é comutativa e $\omega(\mathrm{x} \star \mathrm{y})=\omega(\mathrm{x}) \omega(\mathrm{y})$, por (4.24). 
(b) Se e $\in$ A é idempotente então e $\star \mathrm{e}=(1-2 \gamma) \mathrm{e}^{2}+\gamma[\omega(\mathrm{e}) \mathrm{e}+\mathrm{e} \omega(\mathrm{e})]=$ $=(1-2 \gamma) \mathrm{e}+2 \gamma \mathrm{e}=\mathrm{e}$. Reciprocamente, $\mathrm{e} \star \mathrm{e}=\mathrm{e}$ implica que $(1-2 \gamma) \mathrm{e}^{2}=$ $=\mathrm{e} \star \mathrm{e}-2 \gamma \mathrm{e}=\mathrm{e}-2 \gamma \mathrm{e}=(1-2 \gamma) \mathrm{e}$. Logo $\mathrm{e}^{2}=\mathrm{e}$.

(c) Seja $\mathrm{x} \in \mathrm{A}_{1}$ de peso 1. Então $\mathrm{x} \star \mathrm{x}=(1-2 \gamma) \mathrm{x}^{2}+2 \gamma \mathrm{x}$ e portanto $(\mathrm{x} \star \mathrm{x}) \star \mathrm{x}=(1-2 \gamma) \mathrm{x}^{2} \star \mathrm{x}+2 \gamma \mathrm{x} \star \mathrm{x}=(1-2 \gamma)^{2} \mathrm{x}^{3}+(1-2 \gamma) \gamma\left(\mathrm{x}^{2}+\mathrm{x}\right)+$ $+2 \gamma(1-2 \gamma) \mathrm{x}^{2}+2 \gamma(2 \gamma \mathrm{x})=(1-2 \gamma)^{2} \mathrm{x}^{2}+(1-2 \gamma) \gamma \mathrm{x}^{2}+(1-2 \gamma) \gamma \mathrm{x}+$ $+2 \gamma(1-2 \gamma) \mathrm{x}^{2}+4 \gamma^{2} \mathrm{x}=\left[(1-2 \gamma)^{2}+\gamma(1-2 \gamma)+2 \gamma(1-2 \gamma)\right] \mathrm{x}^{2}+$ $+\gamma[(1-2 \gamma)+4 \gamma] \mathrm{x}=\left(1-4 \gamma+4 \gamma^{2}+\gamma-2 \gamma^{2}+2 \gamma-4 \gamma^{2}\right) \mathrm{x}^{2}+$ $+\gamma(1+2 \gamma) \mathrm{x}=\left(1-\gamma-2 \gamma^{2}\right) \mathrm{x}^{2}+\gamma(1+2 \gamma) \mathrm{x}=\left(1-2 \gamma+\gamma-2 \gamma^{2}\right) \mathrm{x}^{2}+$ $+\left(2 \gamma+2 \gamma^{2}-\gamma\right) \mathrm{x}=(1+\gamma)(1-2 \gamma) \mathrm{x}^{2}+2 \gamma(1+\gamma) \mathrm{x}-\gamma \mathrm{x}=$ $=(1+\gamma)\left[(1-2 \gamma) \mathrm{x}^{2}+2 \gamma \mathrm{x}\right]-\gamma \mathrm{x}=(1+\gamma) \mathrm{x} \star \mathrm{x}-\gamma \mathrm{x}$.

(d) Denotemos por $\mathrm{U}^{*}$ e $\mathrm{V}^{*}$ os subespaços de Peirce de $\mathrm{A}_{1}$ relativamente a um idempotente $e$, isto é, $\mathrm{U}^{*}=\{\mathrm{u} \in \mathrm{N}: 2 \mathrm{e} \star \mathrm{u}=\mathrm{u}\}$ e $\mathrm{V}^{*}=\{\mathrm{v} \in$ $\in \mathrm{N}: \mathrm{e} \star \mathrm{v}=\gamma \mathrm{v}\}$. Dessa forma, se $\mathrm{u} \in \mathrm{U}^{*}$ então $\mathrm{u}=\mathrm{u}_{1}+\mathrm{v}_{1}$, com $\mathrm{u}_{1} \in$ $\in \mathrm{U}$ e $\mathrm{v}_{1} \in \mathrm{V}$ é tal que $\mathrm{u}=2 \mathrm{e} \star \mathrm{u}=2 \mathrm{e} \star\left(\mathrm{u}_{1}+\mathrm{v}_{\mathrm{l}}\right)=2 \mathrm{e} \star \mathrm{u}_{1}+2 \mathrm{e} \star \mathrm{v}_{1}=$ $=2\left[(1-2 \gamma) \mathrm{eu}_{1}+\gamma \mathrm{u}_{1}\right]+2\left[(1-2 \gamma) \mathrm{ev}_{1}+\gamma \mathrm{v}_{1}\right]=(1-2 \gamma) \mathrm{u}_{1}+2 \gamma \mathrm{u}_{1}+$ $+2 \gamma \mathrm{v}_{1}=(1-2 \gamma) \mathrm{u}_{1}+2 \gamma \mathrm{u}$ e portanto $(1-2 \gamma) \mathrm{u}=(1-2 \gamma) \mathrm{u}_{1}$, isto é, $\mathrm{u}=$ $=\mathrm{u}_{1}$. Ou seja, $\mathrm{U}^{*}=\mathrm{U}$. Por outro lado, se $\mathrm{v} \in \mathrm{V}^{*}$ então $\mathrm{v}=\mathrm{u}_{2}+\mathrm{v}_{2}$, com $\mathrm{u}_{2} \in \mathrm{U}$ e $\mathrm{v}_{2} \in \mathrm{V}$ e daí segue que $2 \gamma \mathrm{v}=2 \mathrm{e} \star \mathrm{v}=2 \mathrm{e} \star\left(\mathrm{u}_{2}+\mathrm{v}_{2}\right)=2 \mathrm{e} \star \mathrm{u}_{2}+$ $+2 \mathrm{e} \star \mathrm{v}_{2}=2\left[(1-2 \gamma) \mathrm{eu}_{2}+\gamma \mathrm{u}_{2}\right]+2\left[(1-2 \gamma) \mathrm{ev}_{2}+\gamma \mathrm{v}_{2}\right]=(1-2 \gamma) \mathrm{u}_{2}+$ $+2 \gamma \mathrm{u}_{2}+2 \gamma \mathrm{v}_{2}=(1-2 \gamma) \mathrm{u}_{2}+2 \gamma \mathrm{v}$ e portanto $(1-2 \gamma) \mathrm{u}_{2}=0$, o que implica em $\mathrm{u}_{2}=0$. Logo, $\mathrm{v}=\mathrm{v}_{2}$ e assim $\mathrm{V}=\mathrm{V}^{*}$.

\subsection{Resultados e exemplos}

Nesta seção estudaremos a influência dos P-monômios de grau $\leq 4$ sobre a dimensão de A. Consideramos situações nas quais um determinado P-monômio é não nulo e obtivemos, em cada caso, um valor mínimo para a dimensão de A. Cada um dos resultados obtidos pode ser lido ao contrário. Por exemplo, o Lema 4.9 diz que para todas as t-álgebras de dimensão $\leq 10$ temos $\mathrm{U}^{-1}=0$. Além disso, para cada caso encontramos 
um exemplo minimal. A busca dos exemplos minimais é uma tarefa mais interessante do que estimar a mínima dimensão de A pois não há métodos gerais e devemos nos guiar um pouco pela intuição. Ao exibir os exemplos minimais, não provamos que a álgebra dada é efetivamente train, pois isto é uma tarefa rotineira (porém longa).

Para que o leitor possa ter uma idéia de como foram obtidos os exemplos minimais aqui apresentados, descrevemos rapidamente como encontramos o Exemplo 10, no qual queríamos $\mathrm{U}^{3} \mathrm{~V} \neq 0$ (os outros exemplos foram contruidos de maneira análoga). Pelo Lema 4.10, $\mathrm{U}^{3} \mathrm{~V} \neq 0$ implica na existência de elementos $\mathrm{u}_{1}, \mathrm{u}_{2} \in \mathrm{U}$ e $\mathrm{v} \in \mathrm{V}$ tais que $\left(\mathrm{u}_{1}^{2} \mathrm{u}_{2}\right) \mathrm{v} \neq 0$ e além disso $\left\{\mathrm{u}_{1}, \mathrm{u}_{2}, \mathrm{u}_{1} \mathrm{v}, \mathrm{u}_{2} \mathrm{v}, \mathrm{u}_{1}^{2} \mathrm{u}_{2}, \mathrm{u}_{1}^{2}\left(\mathrm{u}_{2} \mathrm{v}\right), \mathrm{u}_{1}^{2}, \mathrm{u}_{1} \mathrm{u}_{2}, \mathrm{u}_{1}\left(\mathrm{u}_{2} \mathrm{v}\right), \mathrm{v}\right\}$ é livre. Consideramos $\mathrm{u}_{3}=\mathrm{u}_{1} \mathrm{v}, \mathrm{u}_{4}=\mathrm{u}_{2} \mathrm{v}, \mathrm{u}_{5}=\mathrm{u}_{1}^{2} \mathrm{u}_{2}, \mathrm{u}_{6}=\mathrm{u}_{1}^{2}\left(\mathrm{u}_{2} \mathrm{v}\right), \mathrm{v}_{1}=\mathrm{u}_{1}^{2}$, $\mathrm{v}_{2}=\mathrm{u}_{1} \mathrm{u}_{2}, \mathrm{v}_{3}=\mathrm{u}_{1}\left(\mathrm{u}_{2} \mathrm{v}\right)$ e $\mathrm{v}_{4}=\mathrm{v}$ e calculamos, usando as relações (1.8) a (1.12), a tábua de multiplicação de uma t-álgebra $\mathrm{A}$ de posto $3 \mathrm{com}$ base $\left\{\mathrm{e}, \mathrm{u}_{1}, \ldots, \mathrm{u}_{6}, \mathrm{v}_{1}, \ldots, \mathrm{v}_{4}\right\}$ (sendo $e$ um elemento idempotente de A de peso 1). Alguns dos produtos desta tábua puderam ser determinados pelas identidades (1.13) a (1.18). Cada produto não conhecido foi escrito como combinação linear da base do subespaço a que ele pertencia e impondo que a álgebra A satisfizesse a equação (1.1) determinamos os coeficientes da referida combinação linear.

Lembremos que estamos considerando fixada uma t-álgebra $\mathrm{A}=\mathrm{Fe} \oplus$ $\oplus \mathrm{U} \oplus \mathrm{V}$ de posto 3 , de tipo $(1+r, s)$. Nos exemplos apresentados, a função peso é definida como valendo 1 sobre $e$ e zero nos demais elementos da base.

Lema 4.4 Se o P-monômio $U^{2}$ é não nulo, então $r \geq 1, s \geq 1$ e portanto $\operatorname{dim} A \geq 3$.

Prova: De $\mathrm{U}^{2} \neq 0$, segue que $\mathrm{U} \neq 0$ e portanto $r \geq 1$. Além disso, como $0 \neq \mathrm{U}^{2} \subseteq \mathrm{V}$, temos que $s \geq 1$ e dessa forma $\operatorname{dim} \mathrm{A} \geq 3$.

Exemplo 1: A álgebra A com base $\{\mathrm{e}, \mathrm{u}, \mathrm{v}\}$ e tábua de multiplicação 


$$
\left\{\begin{array}{l}
\mathrm{e}^{2}=\mathrm{e} ; 2 \mathrm{eu}=\mathrm{u} \\
\mathrm{u}^{2}=\mathrm{v} \\
\text { os outros produtos são nulos }
\end{array}\right.
$$

satisfaz $\mathrm{x}^{3}=\omega(\mathrm{x}) \mathrm{x}^{2}$, com $\mathrm{U}^{2} \neq 0$ e dimensão 3.

Lema 4.5 Se o P-monômio UV é não nulo, então $r \geq 2$ e $s \geq 1$ e portanto $\operatorname{dim} A \geq 4$.

Prova: Sejam $u \in U$ e $v \in V$ tais que $u v \neq 0$. Afirmo que $\{u$, uv $\}$ é livre. De fato, se $\alpha \mathrm{u}+\beta \mathrm{uv}=0$, então $0=\alpha \mathrm{uv}+\beta(\mathrm{uv}) \mathrm{v}=\alpha \mathrm{uv}$ (por (1.15)) e portanto $\alpha=0$, o que implica em $\beta=0$. Logo, $\operatorname{dim} U \geq 2$. Além disso, como $\mathrm{v} \neq 0$, segue que $\operatorname{dim} \mathrm{V} \geq 1 \mathrm{e}$, consequentemente, $\operatorname{dim} \mathrm{A} \geq 4$.

Exemplo 2: A álgebra A com base $\left\{\mathrm{e}, \mathrm{u}_{1}, \mathrm{u}_{2}, \mathrm{v}\right\}$ e tábua de multiplicação

$$
\left\{\begin{array}{l}
\mathrm{e}^{2}=\mathrm{e} ; 2 \mathrm{eu}_{\mathrm{i}}=\mathrm{u}_{\mathrm{i}}(\mathrm{i}=1,2) \\
\mathrm{ev}=0 \\
\mathrm{u}_{1} \mathrm{v}=\mathrm{u}_{2} \\
\text { os outros produtos são nulos }
\end{array}\right.
$$

satisfaz $\mathrm{x}^{3}=\omega(\mathrm{x}) \mathrm{x}^{2}$, com UV $\neq 0$ e dimensão 4.

Corolário 4.5.1 Nas condições do Lema 4.5, se existe um gerador uv de $U V$ tal que $u \notin a n n\{u\}$, então, $r, s \geq 2$ e portanto dim $A \geq 5$.

Prova: Basta ver que, nesse caso, $\left\{\mathrm{u}^{2}, \mathrm{v}\right\}$ é livre, pois se $\alpha \mathrm{u}^{2}+\beta \mathrm{v}=0$, então $\beta \mathrm{uv}=0$ e portanto $\beta=0$. Logo, $\alpha \mathrm{u}^{2}=0$, o que implica em $\alpha=$ $=0$ e $\operatorname{assim} \mathrm{s} \geq 2$ e $\operatorname{dim} \mathrm{A} \geq 5$, uma vez que, pelo Lema $4.5, \mathrm{r} \geq 2$. 
Exemplo 3: A álgebra A com base $\left\{e, u_{1}, u_{2}, u_{1}^{2}, v\right\}$ e tábua de multiplicação

$$
\left\{\begin{array}{l}
\mathrm{e}^{2}=\mathrm{e} ; 2 \mathrm{eu}_{\mathrm{i}}=\mathrm{u}_{\mathrm{i}}(\mathrm{i}=1,2) \\
\mathrm{eu}_{1}^{2}=\mathrm{ev}=0 \\
\mathrm{u}_{1} \mathrm{v}=\mathrm{u}_{2} \\
\text { os outros produtos são nulos }
\end{array}\right.
$$

é uma t-álgebra de posto 3 , satisfazendo $\mathrm{x}^{3}=\omega(\mathrm{x}) \mathrm{x}^{2}$, com $\mathrm{UV} \neq$ $\neq 0, u_{1} \notin \operatorname{ann}\left\{u_{1}\right\}$ e dimensão 5 .

Lema 4.6 Se o P-monômio $U^{3}$ é não nulo, então $r \geq 3$ e $s \geq 2$ e portanto $\operatorname{dim} A \geq 6$.

Prova: Afirmo que existem elementos $u_{1}, u_{2} \in U$ tais que $u_{1}^{2} u_{2} \neq 0$. De fato, se fosse $\mathrm{u}_{1}^{2} \mathrm{u}_{2}=0$ para quaisquer $\mathrm{u}_{1}, \mathrm{u}_{2} \in \mathrm{U}$, então teríamos $2\left(\mathrm{u}_{1} \mathrm{u}_{3}\right) \mathrm{u}_{2}=\left(\mathrm{u}_{1}+\mathrm{u}_{3}\right)^{2} \mathrm{u}_{2}-\mathrm{u}_{1}^{2} \mathrm{u}_{2}-\mathrm{u}_{3}^{2} \mathrm{u}_{2}=0$ para quaisquer $\mathrm{u}_{1}, \mathrm{u}_{2}, \mathrm{u}_{3} \in \mathrm{U}$, contra a hipótese de $\mathrm{U}^{3}$ ser não nulo. Observemos agora que se $\mathrm{u}_{1}^{2} \mathrm{u}_{2} \neq 0$. então $\left\{\mathrm{u}_{1}, \mathrm{u}_{2}\right\}$ é livre. Caso contrário teríamos $\mathrm{u}_{1}=\alpha \mathrm{u}_{2}\left(\mathrm{ou} \mathrm{u}_{2}=\beta \mathrm{u}_{1}\right) \mathrm{e}$ portanto $\mathrm{u}_{1}^{2} \mathrm{u}_{2}=\alpha^{2} \mathrm{u}_{2}^{2} \mathrm{u}_{2}=\alpha^{2} \mathrm{u}_{2}^{3}=0$ (ou, equivalentemente, $\mathrm{u}_{1}^{2} \mathrm{u}_{2}=\beta^{2} \mathrm{u}_{1}^{3}=$ $=0$ ). Mostremos também que $\left\{u_{1}^{2}, u_{1} u_{2}\right\}$ é livre. Se $\alpha_{1} u_{1}^{2}+\alpha_{2} u_{1} u_{2}=$ 0 , então de (4.1) $0=2 \alpha_{2} \mathrm{u}_{1}\left(\mathrm{u}_{1} \mathrm{u}_{2}\right)=-\alpha_{2} \mathrm{u}_{1}^{2} \mathrm{u}_{2}$, o que significa que $\alpha_{2}=0$. e portanto $\alpha_{1}=0$. Assim, $\mathrm{s} \geq 2$. Quanto a $r$, já vimos que $\left\{\mathrm{u}_{1}, \mathrm{u}_{2}\right\} \subseteq$ $\mathrm{U}$ é livre. Afirmamos agora que $\mathrm{u}_{1}^{2} \mathrm{u}_{2}$ não depende linearmente de $\mathrm{u}_{1} \mathrm{e}$ $\mathrm{u}_{2}$, o que garantirá que $\mathrm{r} \geq 3$. De fato, se $\mathrm{u}_{1}^{2} \mathrm{u}_{2}=\alpha \mathrm{u}_{1}+\beta \mathrm{u}_{2}(\alpha, \beta \in$ F), então por (1.18), $0=\mathrm{u}_{1}\left(\mathrm{u}_{1}^{2} \mathrm{u}_{2}\right)=\mathrm{u}_{1}\left(\alpha \mathrm{u}_{1}+\beta \mathrm{u}_{2}\right)=\alpha \mathrm{u}_{1}^{2}+\beta \mathrm{u}_{1} \mathrm{u}_{2} \mathrm{e}$ portanto $\alpha=\beta=0$, pois já vimos acima que $\left\{\mathrm{u}_{1}^{2}, \mathrm{u}_{1} \mathrm{u}_{2}\right\}$ é livre. Ou seja, $\mathrm{r} \geq 3$ e $\operatorname{dim} \mathrm{A} \geq 6$.

Exemplo 4: A álgebra A com base $\left\{e, u_{1}, u_{2}, u_{3}, v_{1}, v_{2}\right\}$ e tábua de multiplicação 


$$
\left\{\begin{array}{l}
\mathrm{e}^{2}=\mathrm{e} ; 2 \mathrm{eu}_{\mathrm{i}}=\mathrm{u}_{\mathrm{i}}(\mathrm{i}=1,2,3) ; \quad \text { ev } \mathrm{i}=0 \quad(\mathrm{i}=1.2) \\
\mathrm{u}_{1}^{2}=\mathrm{v}_{1} ; \quad \mathrm{u}_{1} \mathrm{u}_{3}=\mathrm{v}_{2} ; 2 \mathrm{u}_{1} \mathrm{v}_{2}=-\mathrm{u}_{2} \\
\mathrm{u}_{3}^{2}=-\mathrm{v}_{1}+2 \mathrm{v}_{2} ; \quad \mathrm{u}_{3} \mathrm{v}_{1}=\mathrm{u}_{2} ; 2 \mathrm{u}_{3} \mathrm{v}_{2}=\mathrm{u}_{2} \\
\text { os outros produtos são nulos }
\end{array}\right.
$$

satisfaz $\mathrm{x}^{3}=\omega(\mathrm{x}) \mathrm{x}^{2}, \mathrm{U}^{3} \neq 0\left(\right.$ pois $\left.2 \mathrm{u}_{1}\left(\mathrm{u}_{1} \mathrm{u}_{3}\right)=2 \mathrm{u}_{1} \mathrm{v}_{2}=-\mathrm{u}_{2}\right)$ e sua dimensão é 6 .

Lema 4.7 Se o P-monômio $U(U V)$ é não nulo, então $r \geq 4, s \geq 2$ (e portanto $\operatorname{dim} A \geq 7$ ).

Prova: Tomemos $\mathrm{u}_{1}, \mathrm{u}_{2} \in \mathrm{U}, \mathrm{v}_{1} \in \mathrm{V}$ tais que $\mathrm{u}_{2}\left(\mathrm{u}_{1} \mathrm{v}_{1}\right) \neq 0$. Mostremos, inicialmente, que $\left\{\mathrm{u}_{1}, \mathrm{u}_{2}, \mathrm{u}_{1} \mathrm{v}_{1}, \mathrm{u}_{2} \mathrm{v}_{1}\right\} \subseteq \mathrm{U}$ é livre. Se $\alpha_{1} \mathrm{u}_{1}+\alpha_{2} \mathrm{u}_{2}+$ $+\alpha_{3} \mathrm{u}_{1} \mathrm{v}_{1}+\alpha_{4} \mathrm{u}_{2} \mathrm{v}_{1}=0$, então multiplicando por $\mathrm{v}_{1}$, de (1.15) obtemos $\alpha_{1} \mathrm{u}_{1} \mathrm{v}_{1}+\alpha_{2} \mathrm{u}_{2} \mathrm{v}_{1}=0$ e portanto (por (1.14)) $\alpha_{1} \mathrm{u}_{2}\left(\mathrm{u}_{1} \mathrm{v}_{1}\right)=0$, o que implica em $\alpha_{1}$ e $\alpha_{2}$ serem ambos nulos (note que aqui ficou mostrado que $\left\{u_{1} v_{1}\right.$, $\left.\mathrm{u}_{2} \mathrm{v}_{1}\right\}$ é livre). Logo, $\alpha_{3} \mathrm{u}_{1} \mathrm{v}_{1}+\alpha_{4} \mathrm{u}_{2} \mathrm{v}_{1}=0$, o que significa que $\alpha_{3}$ e $\alpha_{4}$ são nulos e $\operatorname{dim} \mathrm{U} \geq 4$. Mostremos que $\left\{\mathrm{v}_{1}, \mathrm{u}_{2}\left(\mathrm{u}_{1} \mathrm{v}_{1}\right)\right\} \subseteq \mathrm{V}$ é livre. Para isso, suponhamos que $\beta_{1} \mathrm{v}_{1}+\beta_{2} \mathrm{u}_{2}\left(\mathrm{u}_{1} \mathrm{v}_{1}\right)=0$. Então, multiplicando por $2 \mathrm{u}_{2} \mathrm{e}$ de (4.1), $0=2 \beta_{1} \mathrm{u}_{2} \mathrm{v}_{1}+2 \beta_{2} \mathrm{u}_{2}\left[\mathrm{u}_{2}\left(\mathrm{u}_{1} \mathrm{v}_{1}\right)\right]=2 \beta_{1} \mathrm{u}_{2} \mathrm{v}_{1}-\beta_{2} \mathrm{u}_{2}^{2}\left(\mathrm{u}_{1} \mathrm{v}_{1}\right)$ e portanto $0=2 \beta_{1} \mathrm{u}_{1}\left(\mathrm{u}_{2} \mathrm{v}_{1}\right)-\beta_{2} \mathrm{u}_{1}\left[\mathrm{u}_{2}^{2}\left(\mathrm{u}_{1} \mathrm{v}_{1}\right)\right]=-2 \beta_{1} \mathrm{u}_{2}\left(\mathrm{u}_{1} \mathrm{v}_{1}\right)+\beta_{2}\left(\mathrm{u}_{1} \mathrm{v}_{1}\right)\left(\mathrm{u}_{1} \mathrm{u}_{2}^{2}\right)=$ $=-2 \beta_{1} \mathrm{u}_{2}\left(\mathrm{u}_{1} \mathrm{v}_{1}\right)$, sendo que as duas últimas igualdades vêm de (4.4), (4.5) e (4.9), respectivamente. Portanto $\beta_{1}=\beta_{2}=0$ e $\operatorname{dim} \mathrm{V} \geq 2$.

Exemplo 5: A álgebra A com base $\left\{e, u_{1}, u_{2}, u_{3}, u_{4}, v_{1}, v_{2}\right\}$ e tábua de multiplicação

$$
\left\{\begin{array}{l}
\mathrm{e}^{2}=\mathrm{e} ; 2 \mathrm{eu}_{\mathrm{i}}=\mathrm{u}_{\mathrm{i}}(\mathrm{i}=1, \ldots, 4) ; \quad \mathrm{v}_{\mathrm{i}}=0 \quad(\mathrm{i}=1,2) \\
-\mathrm{u}_{1} \mathrm{u}_{4}=\mathrm{u}_{2} \mathrm{u}_{3}=\mathrm{v}_{2} \\
\mathrm{u}_{1} \mathrm{v}_{1}=\mathrm{u}_{3}: \mathrm{u}_{2} \mathrm{v}_{1}=\mathrm{u}_{4} \\
\text { os outros produtos são nulos }
\end{array}\right.
$$


satisfaz $\mathrm{x}^{3}=\omega(\mathrm{x}) \mathrm{x}^{2}, \operatorname{com} \mathrm{U}(\mathrm{UV}) \neq 0\left(\right.$ pois $0 \neq \mathrm{v}_{2}=\mathrm{u}_{2} \mathrm{u}_{3}=\mathrm{u}_{2}\left(\mathrm{u}_{1} \mathrm{v}_{1}\right) \epsilon$ $\in \mathrm{U}(\mathrm{UV}))$ e dimensão $\tau$.

Corolário 4.7.1 Nas condições do Lema 4.7, se $U(U V)$ possui um gerador aditivo $u_{2}\left(u_{1} v_{1}\right)$ tal que $u_{2}^{2} \notin a n n\left\{u_{1} v_{1}\right\}$, então $r \geq 6, s \geq 4 e$ portanto $\operatorname{dim} A \geq 11$.

Prova: Nesse caso, o conjunto $\left\{\mathrm{v}_{1}, \mathrm{u}_{2}\left(\mathrm{u}_{1} \mathrm{v}_{1}\right), \mathrm{u}_{2}^{2}, \mathrm{u}_{1} \mathrm{u}_{2}\right\} \subseteq \mathrm{V}$ é livre. Supondo $\alpha_{1} \mathrm{v}_{1}+\alpha_{2} \mathrm{u}_{2}\left(\mathrm{u}_{1} \mathrm{v}_{1}\right)+\alpha_{3} \mathrm{u}_{2}^{2}+\alpha_{4} \mathrm{u}_{1} \mathrm{u}_{2}=0$, multiplicando por $2 \mathrm{u}_{2}$, segue que $0=2 \alpha_{1} \mathrm{u}_{2} \mathrm{v}_{1}+2 \alpha_{2} \mathrm{u}_{2}\left[\mathrm{u}_{2}\left(\mathrm{u}_{1} \mathrm{v}_{1}\right)\right]+2 \alpha_{4} \mathrm{u}_{2}\left(\mathrm{u}_{1} \mathrm{u}_{2}\right)=2 \alpha_{1} \mathrm{u}_{2} \mathrm{v}_{1}+$ $-\alpha_{2} \mathrm{u}_{2}^{2}\left(\mathrm{u}_{1} \mathrm{v}_{1}\right)-\alpha_{4} \mathrm{u}_{2}^{2} \mathrm{u}_{1}$, por (4.1). Logo, $0=-\alpha_{4}\left(\mathrm{u}_{2}^{2} \mathrm{u}_{1}\right) \mathrm{v}_{1}=\alpha_{4} \mathrm{u}_{2}^{2}\left(\mathrm{u}_{1} \mathrm{v}_{1}\right)$ (por (4.3) e (1.15)) e daí segue que $\alpha_{4}=0$ e $2 \alpha_{1} \mathrm{u}_{2} \mathrm{v}_{1}-\alpha_{2} \mathrm{u}_{2}^{2}\left(\mathrm{u}_{1} \mathrm{v}_{1}\right)=0$. Aqui, como no Lema 4.7, teremos $\alpha_{1}=\alpha_{2}=0$ e, portanto, $\alpha_{3}=0$, o que implica em $\operatorname{dim} \mathrm{V} \geq 4$. Afirmamos que $\left\{\mathrm{u}_{1}, \mathrm{u}_{2}, \mathrm{u}_{1} \mathrm{v}_{1}, \mathrm{u}_{2} \mathrm{v}_{1}, \mathrm{u}_{2}^{2} \mathrm{u}_{1}, \mathrm{u}_{2}^{2}\left(\mathrm{u}_{1} \mathrm{v}_{1}\right)\right\}$ é livre. Para simplificar nossos cálculos, comecemos provando que $\left\{\mathrm{u}_{1} \mathrm{v}_{1}, \mathrm{u}_{2} \mathrm{v}_{1}\right.$, $\left.\mathrm{u}_{2}^{2}\left(\mathrm{u}_{1} \mathrm{v}_{1}\right)\right\}$ é livre. Se $\lambda_{1} \mathrm{u}_{1} \mathrm{v}_{1}+\lambda_{2} \mathrm{u}_{2} \mathrm{v}_{1}+\lambda_{3} \mathrm{u}_{2}^{2}\left(\mathrm{u}_{1} \mathrm{v}_{1}\right)=0$, multiplicando por $\mathrm{u}_{2}^{2}$ e de (1.15) e (1.16), obtemos então $\lambda_{1} \mathrm{u}_{2}^{2}\left(\mathrm{u}_{1} \mathrm{v}_{1}\right)=0$, isto é, $\lambda_{1}=$ $=0$ e $\lambda_{2} \mathrm{u}_{2} \mathrm{v}_{1}+\lambda_{3} \mathrm{u}_{2}^{2}\left(\mathrm{u}_{1} \mathrm{v}_{1}\right)=0$. Logo, multiplicando por $\mathrm{u}_{1}$, segue que $0=$ $=\lambda_{2} \mathrm{u}_{1}\left(\mathrm{u}_{2} \mathrm{v}_{1}\right)+\lambda_{3} \mathrm{u}_{1}\left[\mathrm{u}_{2}^{2}\left(\mathrm{u}_{1} \mathrm{v}_{1}\right)\right]=\lambda_{2} \mathrm{u}_{1}\left(\mathrm{u}_{2} \mathrm{v}_{1}\right)=-\lambda_{2} \mathrm{u}_{2}\left(\mathrm{u}_{1} \mathrm{v}_{1}\right)$, pois, por $(4.4)$ e (4.9), $\mathrm{u}_{1}\left[\mathrm{u}_{2}^{2}\left(\mathrm{u}_{1} \mathrm{v}_{1}\right)\right]=0$ e a última igualdade segue de (4.4). Isto implica em $\lambda_{2}=0$, uma vez que $0 \neq \mathrm{u}_{2}^{2}\left(\mathrm{u}_{1} \mathrm{v}_{1}\right)=-2 \mathrm{u}_{2}\left[\mathrm{u}_{2}\left(\mathrm{u}_{1} \mathrm{v}_{1}\right)\right]$. Portanto, $\lambda_{3}=$ $=0$, o que completa a prova de que $\left\{u_{1} v_{1}, u_{2} v_{1}, u_{2}^{2}\left(u_{1} v_{1}\right)\right\}$ é livre. Finalmente, se $\beta_{1} u_{1}+\beta_{2} u_{2}+\beta_{3} u_{1} v_{1}+\beta_{4} u_{2} v_{1}+\beta_{5} u_{2}^{2} u_{1}+\beta_{6} u_{2}^{2}\left(u_{1} v_{1}\right)=0$. então, multiplicando por $\mathrm{v}_{1}$ e de (1.15), obtemos $0=\beta_{1} \mathrm{u}_{1} \mathrm{v}_{1}+\beta_{2} \mathrm{u}_{2} \mathrm{v}_{1}+$ $+\beta_{5}\left(\mathrm{u}_{2}^{2} \mathrm{u}_{1}\right) \mathrm{v}_{1}=\beta_{1} \mathrm{u}_{1} \mathrm{v}_{1}+\beta_{2} \mathrm{u}_{2} \mathrm{v}_{1}-\beta_{5} \mathrm{u}_{2}^{2}\left(\mathrm{u}_{1} \mathrm{v}_{1}\right)$ (por (4.3)) e portanto $\beta_{1}$ $=\beta_{2}=\beta_{5}=0$. Ficamos então com $\beta_{3} \mathrm{u}_{1} \mathrm{v}_{1}+\beta_{4} \mathrm{u}_{2} \mathrm{v}_{1}+\beta_{6} \mathrm{u}_{2}^{2}\left(\mathrm{u}_{1} \mathrm{v}_{1}\right)=0 \mathrm{e}$ isto implica em $\beta_{3}=\beta_{4}=\beta_{6}=0$. Dessa forma, $\operatorname{dim} U \geq 6$ e portanto $\operatorname{dim} \mathrm{A} \geq 11$.

Exemplo 6: A álgebra A com base $\left\{e, u_{1}, u_{2}, u_{3}, u_{4}, u_{5}, u_{6}, v_{1}, v_{2}, v_{3}\right.$, $\mathrm{v}_{4}$ \}, e tábua de multiplicação 


$$
\left\{\begin{array}{l}
\mathrm{e}^{2}=\mathrm{e} ; 2 \mathrm{eu}_{\mathrm{i}}=\mathrm{u}_{\mathrm{i}}(\mathrm{i}=1, \ldots, 6) ; \quad \text { ev } \mathrm{i}=0 \quad(\mathrm{i}=1, \ldots, 4) \\
\mathrm{u}_{1} \mathrm{u}_{2}=\mathrm{v}_{4} ;-\mathrm{u}_{1} \mathrm{u}_{4}=\mathrm{u}_{2} \mathrm{u}_{3}=\mathrm{v}_{2} ; \mathrm{u}_{2}^{2}=\mathrm{v}_{3} \\
\mathrm{u}_{1} \mathrm{v}_{1}=\mathrm{u}_{3} ; \quad \mathrm{u}_{2} \mathrm{v}_{1}=\mathrm{u}_{4} ; \quad \mathrm{u}_{1} \mathrm{v}_{3}=-2 \mathrm{u}_{2} \mathrm{v}_{4}=\mathrm{u}_{5} \\
-2 \mathrm{u}_{2} \mathrm{v}_{2}=\mathrm{u}_{3} \mathrm{v}_{3}=-2 \mathrm{u}_{4} \mathrm{v}_{4}=-\mathrm{u}_{5} \mathrm{v}_{1}=\mathrm{u}_{6} \\
\text { os outros produtos são nulos }
\end{array}\right.
$$

satisfaz $\mathrm{x}^{3}=\omega(\mathrm{x}) \mathrm{x}^{2}$, com $\mathrm{U}(\mathrm{UV}) \neq 0\left(\right.$ pois $\left.\mathrm{u}_{2}\left(\mathrm{u}_{1} \mathrm{v}_{1}\right)=\mathrm{u}_{2} \mathrm{u}_{3}=\mathrm{v}_{2}\right), \mathrm{u}_{2}^{2} \notin$ $\notin \operatorname{ann}\left\{\mathrm{u}_{1} \mathrm{v}_{1}\right\}\left(\right.$ pois $\left.\mathrm{u}_{2}^{2}\left(\mathrm{u}_{1} \mathrm{v}_{1}\right)=\mathrm{v}_{3} \mathrm{u}_{3}=\mathrm{u}_{6}\right)$ e dimensão 11 .

Lema 4.8 Se o P-monômio $(U V) V$ é não nulo, então $r \geq 4, s \geq 2$ e portanto $\operatorname{dim} A \geq 7$.

Prova: Consideremos um gerador aditivo (uv) $v_{1}$ de (UV)V. Os elementos v e $\mathrm{v}_{1}$ são livres pois, caso contrário, teríamos (uv) $\mathrm{v}_{1}=0$ por (1.15); ou seja, $\operatorname{dim} V \geq 2$. Mostremos que o subconjunto $\left\{u, u v, u v_{1},(u v) v_{1}\right\}$ de $\mathrm{U}$ é livre. Se $\alpha_{1} \mathrm{u}+\alpha_{2} \mathrm{uv}+\alpha_{3} \mathrm{uv}_{1}+\alpha_{4}(\mathrm{uv}) \mathrm{v}_{1}=0$ então, multiplicando por v e de (1.15), segue que $0=\alpha_{1} u v+\alpha_{3}\left(u_{1}\right) v=\alpha_{1} u v-\alpha_{3}(u v) v_{1}$ (por (4.3)) e portanto $\alpha_{1}(\mathrm{uv}) \mathrm{v}_{1}=0$. Daí segue que $\alpha_{1}=\alpha_{3}=0$ e portanto $\alpha_{2} \mathrm{uv}+\alpha_{4}(\mathrm{uv}) \mathrm{v}_{1}=0$. Logo, $\alpha_{2}(\mathrm{uv}) \mathrm{v}_{1}=0$, o que implica em $\alpha_{2}$ e $\alpha_{4}$ serem ambos nulos. Ou seja, $\operatorname{dim} U \geq 4$ e $\operatorname{dim} A \geq 7$.

Exemplo 7: A álgebra A com base $\left\{e, u_{1}, u_{2}, u_{3}, u_{4}, v_{1}, v_{2}\right\}$ e tábua de multiplicação

$$
\left\{\begin{array}{l}
\mathrm{e}^{2}=\mathrm{e} ; 2 \mathrm{eu}_{\mathrm{i}}=\mathrm{u}_{\mathrm{i}}(\mathrm{i}=1, \ldots, 4) ; \quad \mathrm{ev}_{\mathrm{i}}=0(\mathrm{i}=1,2) \\
\mathrm{u}_{1} \mathrm{v}_{1}=\mathrm{u}_{2} ; \quad \mathrm{u}_{1} \mathrm{v}_{2}=\mathrm{u}_{3} ; \quad \mathrm{u}_{2} \mathrm{v}_{2}=\mathrm{u}_{4} ; \quad \mathrm{u}_{3} \mathrm{v}_{1}=-\mathrm{u}_{4} \\
\text { os outros produtos são nulos }
\end{array}\right.
$$

satisfaz $\mathrm{x}^{3}=\omega(\mathrm{x}) \mathrm{x}^{2}, \operatorname{com}(\mathrm{UV}) \mathrm{V} \neq 0\left(\right.$ pois $\left.0 \neq-\mathrm{u}_{4}=\mathrm{u}_{3} \mathrm{v}_{1}=\left(\mathrm{u}_{1} \mathrm{v}_{2}\right) \mathrm{v}_{1}\right) \mathrm{e}$ dimensão 7 . 
Corolário 4.8.1 Nas condiçôes do Lema 4.8, se o subespaço (UV)V possui um gerador aditivo $(u v) v_{1}$ tal que $u \notin a n n\{u\}$, então $s \geq 3 e$ portanto $\operatorname{dim} A \geq 8$.

Prova: Se $\alpha_{1} \mathrm{u}^{2}+\alpha_{2} \mathrm{v}+\alpha_{3} \mathrm{v}_{1}=0$, então $\alpha_{2} \mathrm{uv}+\alpha_{3} \mathrm{uv}_{1}=0$ e como, pelo Lema anterior, $\left\{\mathrm{uv}, \mathrm{uv}_{1}\right\}$ é livre, segue que $\alpha_{2}=\alpha_{3}=0$ e consequentemente $\alpha_{1}=0$. Assim, $\operatorname{dim} \mathrm{V} \geq 3$ e como, pelo Lema 4.7, $\operatorname{dim} U \geq 4$, segue o resultado.

Exemplo 8: A álgebra A com base $\left\{\mathrm{e}, \mathrm{u}_{1}, \mathrm{u}_{2}, \mathrm{u}_{3}, \mathrm{u}_{4}, \mathrm{v}_{1}, \mathrm{v}_{2}, \mathrm{u}_{1}^{2}\right\}$ e tábua de multiplicação

$$
\left\{\begin{array}{l}
\mathrm{e}^{2}=\mathrm{e} ; 2 \mathrm{eu}_{\mathrm{i}}=\mathrm{u}_{\mathrm{i}}(\mathrm{i}=1, \ldots, 4,) \\
\mathrm{eu}_{1}^{2}=\mathrm{ev}_{\mathrm{i}}=0(\mathrm{i}=1.2) \\
\mathrm{u}_{1} \mathrm{v}_{1}=\mathrm{u}_{2} ; \quad \mathrm{u}_{1} \mathrm{v}_{2}=\mathrm{u}_{3} \\
\mathrm{u}_{2} \mathrm{v}_{2}=\mathrm{u}_{4} ; \quad \mathrm{u}_{3} \mathrm{v}_{1}=-\mathrm{u}_{4} \\
\text { os outros produtos são nulos }
\end{array}\right.
$$

satisfaz $\mathrm{x}^{3}=\omega(\mathrm{x}) \mathrm{x}^{2}, \quad \operatorname{com}(\mathrm{UV}) \mathrm{V} \neq 0 \quad\left(\right.$ pois $0 \neq-\mathrm{u}_{4}=\mathrm{u}_{3} \mathrm{v}_{1}=$ $\left.=\left(\mathrm{u}_{1} \mathrm{v}_{2}\right) \mathrm{v}_{1}\right), \mathrm{u}_{1}^{2} \neq 0$ e dimensão 8 .

Lema 4.9 Se o P-monômio $U^{4}$ é nẫo nulo, então $r \geq 6, s \geq 4$ e portanto $\operatorname{dim} A \geq 11$.

Prova: Afirmo que se $\mathrm{U}^{4} \neq 0$, então existem $\mathrm{u}_{1}, \mathrm{u}_{2}, \mathrm{u}_{3} \in \mathrm{U}$ tais que $\left(u_{1}^{2} u_{2}\right) u_{3} \neq 0$. De fato, se fosse $\left(u_{1}^{2} u_{2}\right) u_{3}=0$ para todos $u_{1}, u_{2}, u_{3} \in$ $\in U$, então $2 u_{4}\left[u_{5}\left(u_{6} u_{7}\right)\right]=u_{4}\left[u_{5}\left(u_{6}+u_{7}\right)^{2}\right]-u_{4}\left(u_{5} u_{6}^{2}\right)-u_{4}\left(u_{5} u_{7}^{2}\right)=0$, contra a hipótese de $U^{4} \neq 0$. Sejam $u_{1}, u_{2}, u_{3} \in U$ tais que $0 \neq\left(u_{1}^{2} u_{2}\right) u_{3}=$ $=-\left(\mathrm{u}_{1}^{2} \mathrm{u}_{3}\right) \mathrm{u}_{2}$ (por (4.4)) e mostremos que o subconjunto $\left\{\mathrm{u}_{1}, \mathrm{u}_{2}, \mathrm{u}_{3}, \mathrm{u}_{1}^{2} \mathrm{u}_{2}\right.$, $\left.\mathrm{u}_{1}^{2} \mathrm{u}_{3},\left(\mathrm{u}_{1} \mathrm{u}_{2}\right) \mathrm{u}_{3}\right\}$ de $\mathrm{U}$ é livre. Para isso, suponhamos $\alpha_{1} \mathrm{u}_{1}+\alpha_{2} \mathrm{u}_{2}+$ $+\alpha_{3} \mathrm{u}_{3}+\alpha_{4} \mathrm{u}_{1}^{2} \mathrm{u}_{2}+\alpha_{5} \mathrm{u}_{1}^{2} \mathrm{u}_{3}+\alpha_{6}\left(\mathrm{u}_{1} \mathrm{u}_{2}\right) \mathrm{u}_{3}=0$. Dessa forma, multiplicando por $\mathrm{u}_{1}^{2}$, temos $\alpha_{2} \mathrm{u}_{1}^{2} \mathrm{u}_{2}+\alpha_{3} \mathrm{u}_{1}^{2} \mathrm{u}_{3}+\alpha_{6} \mathrm{u}_{1}^{2}\left[\left(\mathrm{u}_{1} \mathrm{u}_{2}\right) \mathrm{u}_{3}\right]=0$, por (1.15). 
e portanto de (1.14), $0=\alpha_{2}\left(\mathrm{u}_{1}^{2} \mathrm{u}_{2}\right) \mathrm{u}_{3}+\alpha_{6}\left\{\mathrm{u}_{1}^{2}\left[\left(\mathrm{u}_{1} \mathrm{u}_{2}\right) \mathrm{u}_{3}\right]\right\} \mathrm{u}_{3}=\alpha_{2}\left(\mathrm{u}_{1}^{2} \mathrm{u}_{2}\right) \mathrm{u}_{3}$ (sendo esta última igualdade consequência de (4.12)), o que implica em $\alpha_{2}=0$. Daí segue que $\alpha_{3} \mathrm{u}_{1}^{2} \mathrm{u}_{3}+\alpha_{6} \mathrm{u}_{1}^{2}\left[\left(\mathrm{u}_{1} \mathrm{u}_{2}\right) \mathrm{u}_{3}\right]=0$ e portanto $0=$ $=\alpha_{3}\left(\mathrm{u}_{1}^{2} \mathrm{u}_{3}\right) \mathrm{u}_{2}+\alpha_{6}\left\{\mathrm{u}_{1}^{2}\left[\left(\mathrm{u}_{1} \mathrm{u}_{2}\right) \mathrm{u}_{3}\right]\right\} \mathrm{u}_{2}=\alpha_{3}\left(\mathrm{u}_{1}^{2} \mathrm{u}_{3}\right) \mathrm{u}_{2}$, novamente de (4.12). Logo $\alpha_{3}=0$ e $\alpha_{1} \mathrm{u}_{1}+\alpha_{4} \mathrm{u}_{1}^{2} \mathrm{u}_{2}+\alpha_{5} \mathrm{u}_{1}^{2} \mathrm{u}_{3}+\alpha_{6}\left(\mathrm{u}_{1} \mathrm{u}_{2}\right) \mathrm{u}_{3}=0$. Assim $\alpha_{1} \mathrm{u}_{1} \mathrm{u}_{3}+$ $+\alpha_{4}\left(\mathrm{u}_{1}^{2} \mathrm{u}_{2}\right) \mathrm{u}_{3}=0($ por $(1.14))$ e daí $0=2 \alpha_{1} \mathrm{u}_{1}\left(\mathrm{u}_{1} \mathrm{u}_{3}\right)+2 \alpha_{4} \mathrm{u}_{1}\left[\left(\mathrm{u}_{1}^{2} \mathrm{u}_{2}\right) \mathrm{u}_{3}\right]=$ $=-\alpha_{1} u_{1}^{2} u_{3}-2 \alpha_{4}\left(u_{1}^{2} u_{2}\right)\left(u_{1} u_{3}\right)$, de (4.1) e (4.6). Multiplicando por $u_{2}$, segue que $0=\alpha_{1}\left(\mathrm{u}_{1}^{2} \mathrm{u}_{3}\right) \mathrm{u}_{2}+2 \alpha_{4} \mathrm{u}_{2}\left[\left(\mathrm{u}_{1}^{2} \mathrm{u}_{2}\right)\left(\mathrm{u}_{1} \mathrm{u}_{3}\right)\right]=\alpha_{1}\left(\mathrm{u}_{1}^{2} \mathrm{u}_{3}\right) \mathrm{u}_{2}$ (por (4.12)) e portanto $\alpha_{1}=0$, o que implica em $\alpha_{4}=0$. Temos então que $\alpha_{5} \mathrm{u}_{1}^{2} \mathrm{u}_{3}+$ $+\alpha_{6}\left(\mathrm{u}_{1} \mathrm{u}_{2}\right) \mathrm{u}_{3}=0$ e $\operatorname{assim} 0=2 \alpha_{6} \mathrm{u}_{1}\left[\left(\mathrm{u}_{1} \mathrm{u}_{2}\right) \mathrm{u}_{3}\right]=-2 \alpha_{6} \mathrm{u}_{3}\left[\left(\mathrm{u}_{1} \mathrm{u}_{2}\right) \mathrm{u}_{1}\right]=$ $=\alpha_{6}\left(\mathrm{u}_{1}^{2} \mathrm{u}_{2}\right) \mathrm{u}_{3}$, por (4.4) e (4.1), respectivamente; ou seja, $\alpha_{6}$ e portanto $\alpha_{5}$ são ambos nulos e $\operatorname{dim} U \geq 6$. Afirmo agora que $\left\{u_{1}^{2}, u_{1} u_{2}, u_{1} u_{3}\right.$, $\left.\left(\mathrm{u}_{1}^{2} \mathrm{u}_{2}\right) \mathrm{u}_{3}\right\} \subseteq \mathrm{V}$ é livre. De fato, se $\beta_{1} \mathrm{u}_{1}^{2}+\beta_{2} \mathrm{u}_{1} \mathrm{u}_{2}+\beta_{3} \mathrm{u}_{1} \mathrm{u}_{3}+\beta_{4}\left(\mathrm{u}_{1}^{2} \mathrm{u}_{2}\right) \mathrm{u}_{3}=$ $=0$, então $0=2 \beta_{2} \mathrm{u}_{1}\left(\mathrm{u}_{1} \mathrm{u}_{2}\right)+2 \beta_{3} \mathrm{u}_{1}\left(\mathrm{u}_{1} \mathrm{u}_{3}\right)+2 \beta_{4} \mathrm{u}_{1}\left[\left(\mathrm{u}_{1}^{2} \mathrm{u}_{2}\right) \mathrm{u}_{3}\right]=-\beta_{2} \mathrm{u}_{1}^{2} \mathrm{u}_{2}+$ - $\beta_{3} \mathrm{u}_{1}^{2} \mathrm{u}_{3}-2 \beta_{4} \mathrm{u}_{1}^{2}\left[\mathrm{u}_{3}\left(\mathrm{u}_{1} \mathrm{u}_{2}\right)\right]$ (por (4.1) e (4.4)) e portanto, de (1.14) e (4.12), segue que $0=\beta_{2}\left(\mathrm{u}_{1}^{2} \mathrm{u}_{2}\right) \mathrm{u}_{3}+2 \beta_{4}\left\{\mathrm{u}_{1}^{2}\left[\mathrm{u}_{3}\left[\left(\mathrm{u}_{1} \mathrm{u}_{2}\right)\right]\right\} \mathrm{u}_{3}=\beta_{2}\left(\mathrm{u}_{1}^{2} \mathrm{u}_{2}\right) \mathrm{u}_{3}\right.$. Dessa forma, $\beta_{2}=0$ e $\beta_{3} \mathrm{u}_{1}^{2} \mathrm{u}_{3}+2 \beta_{4} \mathrm{u}_{1}^{2}\left[\mathrm{u}_{3}\left(\mathrm{u}_{1} \mathrm{u}_{2}\right)\right]=0$. Multiplicando por $\mathrm{u}_{2}$, segue que $0=\beta_{3}\left(\mathrm{u}_{1}^{2} \mathrm{u}_{3}\right) \mathrm{u}_{2}+2 \beta_{4} \mathrm{u}_{2}\left\{\mathrm{u}_{1}^{2}\left[\mathrm{u}_{3}\left(\mathrm{u}_{1} \mathrm{u}_{2}\right)\right]\right\}=\beta_{3}\left(\mathrm{u}_{1}^{2} \mathrm{u}_{3}\right) \mathrm{u}_{2}$, por (4.12). Daí segue que $\beta_{3}=0$ e assim $\beta_{1} \mathrm{u}_{1}^{2}+\beta_{4}\left(\mathrm{u}_{1}^{2} \mathrm{u}_{2}\right) \mathrm{u}_{3}=0$. Logo, $0=$ $=\beta_{1} \mathrm{u}_{1}^{2} \mathrm{u}_{2}+\beta_{4}\left[\left(\mathrm{u}_{1}^{2} \mathrm{u}_{2}\right) \mathrm{u}_{3}\right] \mathrm{u}_{2}$; isto é, $0=\beta_{1}\left(\mathrm{u}_{1}^{2} \mathrm{u}_{2}\right) \mathrm{u}_{3}+\beta_{4}\left\{\left[\left(\mathrm{u}_{1}^{2} \mathrm{u}_{2}\right) \mathrm{u}_{3}\right] \mathrm{u}_{2}\right\} \mathrm{u}_{3}=$ $=\beta_{1}\left(\mathrm{u}_{1}^{2} \mathrm{u}_{2}\right) \mathrm{u}_{3}$ (por (4.13)). Dessa forma, $\beta_{1}$ e $\beta_{4}$. são ambos nulos e portanto $\operatorname{dim} \mathrm{V} \geq 4$. Assim $\operatorname{dim} \mathrm{A} \geq 11$.

Exemplo 9: A álgebra A com base $\left\{e, u_{1}, u_{2}, u_{3}, u_{4}, u_{5}, u_{6}, v_{1}, v_{2}, v_{3}\right.$. $\mathrm{v}_{4}$ \} e tábua de multiplicação

$$
\left\{\begin{array}{l}
\mathrm{e}^{2}=\mathrm{e} ; 2 \mathrm{eu}_{\mathrm{i}}=\mathrm{u}_{\mathrm{i}}(\mathrm{i}=1, \ldots, 6) ; \quad \mathrm{ev}_{\mathrm{i}}=0 \quad(\mathrm{i}=1, \ldots, 4) \\
\mathrm{u}_{1}^{2}=\mathrm{v}_{1} ; \quad \mathrm{u}_{1} \mathrm{u}_{2}=\mathrm{v}_{2} ; \quad \mathrm{u}_{1} \mathrm{u}_{3}=\mathrm{v}_{3} \\
2 \mathrm{u}_{1} \mathrm{u}_{6}=-\mathrm{u}_{2} \mathrm{u}_{5}=\mathrm{u}_{3} \mathrm{u}_{4}=\mathrm{v}_{4} \\
-2 \mathrm{u}_{1} \mathrm{v}_{2}=\mathrm{u}_{2} \mathrm{v}_{1}=\mathrm{u}_{4}:-2 \mathrm{u}_{1} \mathrm{v}_{3}=\mathrm{u}_{3} \mathrm{v}_{1}=\mathrm{u}_{5}: \quad-\mathrm{u}_{2} \mathrm{v}_{3}=\mathrm{u}_{3} \mathrm{v}_{2}=\mathrm{u}_{6} \\
\text { os outros produtos são nulos }
\end{array}\right.
$$


satisfaz $\mathrm{x}^{3}=\omega(\mathrm{x}) \mathrm{x}^{2}$, com $\mathrm{U}^{4} \neq 0\left(\right.$ pois $\mathrm{u}_{3}\left(\mathrm{u}_{1}^{2} \mathrm{u}_{2}\right)=\mathrm{u}_{3}\left(\mathrm{u}_{2} \mathrm{v}_{1}\right)=\mathrm{u}_{3} \mathrm{u}_{4}=$ $=\mathrm{v}_{4}$ ) e dimensão 11 .

Lema 4.10 Se o P-monômio $U^{3} V$ é não nulo, então $r \geq 6, s \geq 4 e$ assim $\operatorname{dim} A \geq 11$.

Prova: Nessas condições, afirmo que existem $u_{1}, u_{2} \in U$ e $v \in V$ tais que $\left(u_{1}^{2} u_{2}\right) v \neq 0$. De fato, se $\left(u_{1}^{2} u_{2}\right) v=0$ para todos $u_{1}, u_{2} \in U$ e v $\in V$, então teríamos $2\left[\mathrm{u}_{3}\left(\mathrm{u}_{4} \mathrm{u}_{5}\right)\right] \mathrm{v}_{1}=\left[\left(\mathrm{u}_{4}+\mathrm{u}_{5}\right)^{2} \mathrm{u}_{3}\right] \mathrm{v}_{1}-\left(\mathrm{u}_{4}^{2} \mathrm{u}_{3}\right) \mathrm{v}_{1}-\left(\mathrm{u}_{5}^{2} \mathrm{u}_{3}\right) \mathrm{v}_{1}=0$, o que contraria o fato de $\mathrm{U}^{3} \mathrm{~V} \neq 0$. Consideremos, então, $\mathrm{u}_{1}, \mathrm{u}_{2} \in \mathrm{U}$ e $\mathrm{v} \in \mathrm{V}$ tais que $\left(\mathrm{u}_{1}^{2} \mathrm{u}_{2}\right) \mathrm{v} \neq 0$. Note que $\left(\mathrm{u}_{1}^{2} \mathrm{u}_{2}\right) \mathrm{v}=-\mathrm{u}_{1}^{2}\left(\mathrm{u}_{2} \mathrm{v}\right)=2 \mathrm{u}_{1}\left[\mathrm{u}_{1}\left(\mathrm{u}_{2} \mathrm{v}\right)\right]=$ $=-2 \mathrm{u}_{1}\left[\mathrm{u}_{2}\left(\mathrm{u}_{1} \mathrm{v}\right)\right] \neq 0$, por $(4.3),(4.1)$ e $(4.4)$, respectivamente. Afirmamos que $\left\{\mathrm{u}_{1}, \mathrm{u}_{2}, \mathrm{u}_{1} \mathrm{v}, \mathrm{u}_{2} \mathrm{v}, \mathrm{u}_{1}^{2} \mathrm{u}_{2}, \mathrm{u}_{1}^{2}\left(\mathrm{u}_{2} \mathrm{v}\right)\right\}$ é livre. De fato, se tivermos $\alpha_{1} \mathrm{u}_{1}+$ $+\alpha_{2} \mathrm{u}_{2}+\alpha_{3} \mathrm{u}_{1} \mathrm{v}+\alpha_{4} \mathrm{u}_{2} \mathrm{v}+\alpha_{5} \mathrm{u}_{1}^{2} \mathrm{u}_{2}+\alpha_{6} \mathrm{u}_{1}^{2}\left(\mathrm{u}_{2} \mathrm{v}\right)=0$, então, multiplicando por $\mathrm{u}_{2} \mathrm{v}$, obtemos $\alpha_{1} \mathrm{u}_{1}\left(\mathrm{u}_{2} \mathrm{v}\right)=0$ (por (1.14), (4.10), (1.17), (4.9) e (1.14)) e portanto $\alpha_{1}=0$. Assim, $\alpha_{2} \mathrm{u}_{2}+\alpha_{3} \mathrm{u}_{1} \mathrm{v}+\alpha_{4} \mathrm{u}_{2} \mathrm{v}+\alpha_{5} \mathrm{u}_{1}^{2} \mathrm{u}_{2}+\alpha_{6} \mathrm{u}_{1}^{2}\left(\mathrm{u}_{2} \mathrm{v}\right)=$ $=0$. Logo, multiplicando por $\mathrm{u}_{1}^{2}$, de (1.16) e (1.14), $\alpha_{2} \mathrm{u}_{1}^{2} \mathrm{u}_{2}+\alpha_{4} \mathrm{u}_{1}^{2}\left(\mathrm{u}_{2} \mathrm{v}\right)=$ $=0$, o que implica em $\alpha_{2}\left(u_{1}^{2} u_{2}\right) v=0$ (por (4.3) e (1.15)). Dessa forma, $\alpha_{2}$ e $\alpha_{4}$ são ambos nulos e $\alpha_{3} \mathrm{u}_{1} \mathrm{v}+\alpha_{5} \mathrm{u}_{1}^{2} \mathrm{u}_{2}+\alpha_{6} \mathrm{u}_{1}^{2}\left(\mathrm{u}_{2} \mathrm{v}\right)=0$. Logo, novamente por (1.15), $\alpha_{5}\left(\mathrm{u}_{1}^{2} \mathrm{u}_{2}\right) \mathrm{v}=0$; ou seja, $\alpha_{5}=0$. Segue então que $\alpha_{3} \mathrm{u}_{1} \mathrm{v}+\alpha_{6} \mathrm{u}_{1}^{2}\left(\mathrm{u}_{2} \mathrm{v}\right)=0$, o que implica em $0=\alpha_{3} \mathrm{u}_{2}\left(\mathrm{u}_{1} \mathrm{v}\right)+\alpha_{6} \mathrm{u}_{2}\left[\mathrm{u}_{1}^{2}\left(\mathrm{u}_{2} \mathrm{v}\right)\right]=$ $=\alpha_{3} \mathrm{u}_{2}\left(\mathrm{u}_{1} \mathrm{v}\right)$ (por (4.11)) e portanto $\alpha_{3}$ e $\alpha_{6}$ são ambos nulos. Concluimos então que $\operatorname{dim} U \geq 6$. Mostremos, agora, que $\left\{\mathrm{u}_{1}^{2}, \mathrm{u}_{1} \mathrm{u}_{2}, \mathrm{u}_{1}\left(\mathrm{u}_{2} \mathrm{v}\right), \mathrm{v}\right\}$ é livre e, portanto, $\operatorname{dim} V \geq 4$. Para isso, suponhamos $\beta_{1} u_{1}^{2}+\beta_{2} u_{1} u_{2}+$ $+\beta_{3} \mathrm{u}_{1}\left(\mathrm{u}_{2} \mathrm{v}\right)+\beta_{4} \mathrm{v}=0$. Multiplicando por $2 \mathrm{u}_{1}$, temos $0=2 \beta_{2} \mathrm{u}_{1}\left(\mathrm{u}_{1} \mathrm{u}_{2}\right)+$ $+2 \beta_{3} \mathrm{u}_{1}\left[\mathrm{u}_{1}\left(\mathrm{u}_{2} \mathrm{v}\right)\right]+2 \beta_{4} \mathrm{u}_{1} \mathrm{v}=-\beta_{2} \mathrm{u}_{1}^{2} \mathrm{u}_{2}-\beta_{3} \mathrm{u}_{1}^{2}\left(\mathrm{u}_{2} \mathrm{v}\right)+2 \beta_{4} \mathrm{u}_{1} \mathrm{v}$ (por (4.1)) e como já vimos que $\left\{\mathrm{u}_{1} \mathrm{v}, \mathrm{u}_{1}^{2} \mathrm{u}_{2}, \mathrm{u}_{1}^{2}\left(\mathrm{u}_{2} \mathrm{v}\right)\right\} \subseteq \mathrm{U}$ é livre, segue que $\beta_{2}=\beta_{3}=$ $=\beta_{4}=0$. Logo, $\beta_{1} \mathrm{u}_{1}^{2}=0$, o que implica em $\beta_{1}=0$ : ou seja, $\operatorname{dim} \mathrm{V} \geq 4$ e $\operatorname{dim} \mathrm{A} \geq 11$.

Exemplo 10: A álgebra A com base $\left\{e, u_{1}, u_{2}, u_{3}, u_{4}, u_{5}, u_{6}, v_{1}, v_{2}, v_{3}\right.$, $\mathrm{v}_{4}$ \} e tábua de multiplicação 


$$
\left\{\begin{array}{l}
\mathrm{e}^{2}=\mathrm{e} ; 2 \mathrm{eu}_{\mathrm{i}}=\mathrm{u}_{\mathrm{i}}(\mathrm{i}=1, \ldots, 6) ; \mathrm{ev}_{\mathrm{i}}=0 \quad(\mathrm{i}=1, \ldots, 4) \\
\mathrm{u}_{1}^{2}=\mathrm{v}_{1} ; \mathrm{u}_{2}^{2}=4 \mathrm{v}_{3} \\
\mathrm{u}_{1} \mathrm{u}_{2}=\mathrm{v}_{2} ; \mathrm{u}_{1} \mathrm{u}_{4}=-\mathrm{u}_{2} \mathrm{u}_{3}=\mathrm{v}_{3} \\
-2 \mathrm{u}_{1} \mathrm{v}_{2}=\mathrm{u}_{2} \mathrm{v}_{1}=\mathrm{u}_{5} ; \mathrm{u}_{2} \mathrm{v}_{2}=-2 \mathrm{u}_{3} \mathrm{v}_{2}=\mathrm{u}_{6} \\
-2 \mathrm{u}_{1} \mathrm{v}_{3}=\mathrm{u}_{4} \mathrm{v}_{1}=-\mathrm{u}_{5} \mathrm{v}_{4}=\mathrm{u}_{6} \\
\mathrm{u}_{1} \mathrm{v}_{4}=\mathrm{u}_{3} ; \quad \mathrm{u}_{2} \mathrm{v}_{4}=\mathrm{u}_{4} \\
\text { os outros produtos são nulos }
\end{array}\right.
$$

satisfaz $\mathrm{x}^{3}=\omega(\mathrm{x}) \mathrm{x}^{2}, \operatorname{com} \mathrm{U}^{3} \mathrm{~V} \neq 0\left(\right.$ pois $\left(\mathrm{u}_{1}^{2} \mathrm{u}_{2}\right) \mathrm{v}_{4}=\left(\mathrm{u}_{2} \mathrm{v}_{1}\right) \mathrm{v}_{4}=\mathrm{u}_{5} \mathrm{v}_{4}=$ $=-\mathrm{u}_{6}$ ) e dimensão 11 .

Observação: Nesse exemplo, temos que $2 \mathrm{u}_{1}\left[\mathrm{u}_{2}\left(\mathrm{u}_{1} \mathrm{v}_{4}\right)\right]=2 \mathrm{u}_{1}\left(\mathrm{u}_{2} \mathrm{u}_{3}\right)=$ $=-2 \mathrm{u}_{1} \mathrm{v}_{3}=-\mathrm{u}_{6}$, isto é, $\mathrm{U}[\mathrm{U}(\mathrm{UV})] \neq 0$.

Lema 4.11 Se o P-monômio $U[U(U V)]$ é não nulo, então

(i) se $U^{3} V \neq 0$, então $\operatorname{dim} A \geq 11$.

(ii) se $U^{3} V=0$, então $r \geq 7, s \geq 4$ e portanto $\operatorname{dim} A \geq 12$.

Prova: Se ocorrer (i), o resultado segue do Lema 4.10. Suponhamos, então, que ocorra (ii), isto é, $0=\mathrm{U}^{3} \mathrm{~V}=\mathrm{U}^{2}(\mathrm{UV}$ ) (veja Lema 3.1)). Como $\mathrm{U}[\mathrm{U}(\mathrm{UV})] \neq 0$, existem $\mathrm{u}_{1}, \mathrm{u}_{2}, \mathrm{u}_{3} \in \mathrm{U}$ e $\mathrm{v} \in \mathrm{V}$ tais que $\mathrm{u}_{1}\left[\mathrm{u}_{2}\left(\mathrm{u}_{3} \mathrm{v}\right)\right] \neq 0$. Note que, como $\mathrm{U}^{3} \mathrm{~V}=0$, de $\mathrm{J}\left(\mathrm{u}_{1}, \mathrm{u}_{2}, \mathrm{u}_{3} \mathrm{v}\right)=0$, temos $0 \neq \mathrm{u}_{1}\left[\mathrm{u}_{2}\left(\mathrm{u}_{3} \mathrm{v}\right)\right]=$ $=-\mathrm{u}_{2}\left[\mathrm{u}_{1}\left(\mathrm{u}_{3} \mathrm{v}\right)\right]$. Além disso, $\mathrm{u}_{1} \mathrm{~J}\left(\mathrm{u}_{2}, \mathrm{u}_{3}, \mathrm{v}\right)=\mathrm{u}_{1}\left[\mathrm{u}_{2}\left(\mathrm{u}_{3} \mathrm{v}\right)\right]+\mathrm{u}_{1}\left[\mathrm{u}_{3}\left(\mathrm{u}_{2} \mathrm{v}\right)\right]=$ $=0$, o que implica em $\mathrm{u}_{1}\left[\mathrm{u}_{3}\left(\mathrm{u}_{2} \mathrm{v}\right)\right] \neq 0$ e portanto $\mathrm{u}_{3}\left[\mathrm{u}_{1}\left(\mathrm{u}_{2} \mathrm{v}\right)\right] \neq 0$. Ou seja. $\mathrm{u}_{2}\left(\mathrm{u}_{1} \mathrm{v}\right)=-\mathrm{u}_{1}\left(\mathrm{u}_{2} \mathrm{v}\right) \neq 0$. Afirmo que $\left\{\mathrm{u}_{1}, \mathrm{u}_{2}, \mathrm{u}_{3}, \mathrm{u}_{1} \mathrm{v}, \mathrm{u}_{2} \mathrm{v}, \mathrm{u}_{3} \mathrm{v}, \mathrm{u}_{1}\left[\mathrm{u}_{2}\left(\mathrm{u}_{3} \mathrm{v}\right)\right]\right\}$ é livre. De fato, supondo $\alpha_{1} \mathrm{u}_{1}+\alpha_{2} \mathrm{u}_{2}+\alpha_{3} \mathrm{u}_{3}+\alpha_{4} \mathrm{u}_{1} \mathrm{v}+\alpha_{5} \mathrm{u}_{2} \mathrm{v}+\alpha_{6} \mathrm{u}_{3} \mathrm{v}+$ $+\alpha_{7} \mathrm{u}_{1}\left[\mathrm{u}_{2}\left(\mathrm{u}_{3} \mathrm{v}\right)\right]=0$, então por (1.15) e de $\mathrm{U}^{3} \mathrm{~V}=0, \alpha_{1} \mathrm{u}_{1} \mathrm{v}+\alpha_{2} \mathrm{u}_{2} \mathrm{v}+$ $+\alpha_{3} \mathrm{u}_{3} \mathrm{v}=0$ e assim multiplicando por $\mathrm{u}_{2}$ e de (1.14). temos $\alpha_{1} \mathrm{u}_{2}\left(\mathrm{u}_{1} \mathrm{v}\right)+$ $+\alpha_{3} \mathrm{u}_{2}\left(\mathrm{u}_{3} \mathrm{v}\right)=0$. Logo, multiplicando por $\mathrm{u}_{1}$ e de $\mathrm{J}\left(\mathrm{u}_{1}, \mathrm{u}_{2}, \mathrm{u}_{1} \mathrm{v}\right)=0$, temos $\alpha_{1} \mathrm{u}_{1}\left[\mathrm{u}_{2}\left(\mathrm{u}_{1} \mathrm{v}\right)\right]+\alpha_{3} \mathrm{u}_{1}\left[\mathrm{u}_{2}\left(\mathrm{u}_{3} \mathrm{v}\right)\right]=-\alpha_{1}\left(\mathrm{u}_{1} \mathrm{u}_{2}\right)\left(\mathrm{u}_{1} \mathrm{v}\right)+\alpha_{3} \mathrm{u}_{1}\left[\mathrm{u}_{2}\left(\mathrm{u}_{3} \mathrm{v}\right)\right]=$ 
$=\alpha_{3} \mathrm{u}_{1}\left[\mathrm{u}_{2}\left(\mathrm{u}_{3} \mathrm{v}\right)\right]=0\left(\right.$ pois $\left.\mathrm{U}^{2}(\mathrm{UV})=0\right)$. Dessa forma, $\alpha_{3}=0$ e isto implica em $\alpha_{1}$ e $\alpha_{2}$, serem também nulos (note que aqui ficou mostrado que $\left\{u_{1} v\right.$, $\left.\mathrm{u}_{2} \mathrm{v}, \mathrm{u}_{3} \mathrm{v}\right\}$ é livre). Assim, de $\mathrm{u}_{1}\left[\mathrm{u}_{2}\left(\mathrm{u}_{3} \mathrm{v}\right)\right]=-\mathrm{u}_{2}\left[\mathrm{u}_{1}\left(\mathrm{u}_{3} \mathrm{v}\right)\right]\left(\right.$ pois $\left(\mathrm{u}_{1} \mathrm{u}_{2}\right)\left(\mathrm{u}_{3} \mathrm{v}\right) \in$ $\left.\in \mathrm{U}^{2} \mathrm{~V}\right)$, temos $\alpha_{4} \mathrm{u}_{1} \mathrm{v}+\alpha_{5} \mathrm{u}_{2} \mathrm{v}+\alpha_{6} \mathrm{u}_{3} \mathrm{v}-\alpha_{7} \mathrm{u}_{2}\left[\mathrm{u}_{1}\left(\mathrm{u}_{3} \mathrm{v}\right)\right]=0$, ou seja, $\alpha_{4} \mathrm{u}_{2}\left(\mathrm{u}_{1} \mathrm{v}\right)+\alpha_{6} \mathrm{u}_{2}\left(\mathrm{u}_{3} \mathrm{v}\right)-\alpha_{7} \mathrm{u}_{2}\left\{\mathrm{u}_{2}\left[\mathrm{u}_{1}\left(\mathrm{u}_{3} \mathrm{v}\right)\right]\right\}=\alpha_{4} \mathrm{u}_{2}\left(\mathrm{u}_{1} \mathrm{v}\right)+\alpha_{6} \mathrm{u}_{2}\left(\mathrm{u}_{3} \mathrm{v}\right)=0$, por (1.14). Logo, multiplicando por $\mathrm{u}_{1}, \alpha_{6} \mathrm{u}_{1}\left[\mathrm{u}_{2}\left(\mathrm{u}_{3} \mathrm{v}\right)\right]=0$, uma vez que $\mathrm{u}_{1}\left[\mathrm{u}_{2}\left(\mathrm{u}_{1} \mathrm{v}\right)\right]=-\mathrm{u}_{2}\left[\mathrm{u}_{1}\left(\mathrm{u}_{1} \mathrm{v}\right)\right]-\left(\mathrm{u}_{1} \mathrm{u}_{2}\right)\left(\mathrm{u}_{1} \mathrm{v}\right)=0$, respectivamente por (1.14) e $\mathrm{U}^{2}(\mathrm{UV})=\mathrm{U}^{3} \mathrm{~V}=0$. Isto implica em $\alpha_{6}$ e $\alpha_{4}$ serem ambos nulos. Ficamos então com $\alpha_{5} \mathrm{u}_{2} \mathrm{v}+\alpha_{7} \mathrm{u}_{1}\left[\mathrm{u}_{2}\left(\mathrm{u}_{3} \mathrm{v}\right)\right]=0$ e assim $0=\alpha_{5} \mathrm{u}_{1}\left(\mathrm{u}_{2} \mathrm{v}\right)+$ $+\alpha_{7} \mathrm{u}_{1}\left\{\mathrm{u}_{1}\left[\mathrm{u}_{2}\left(\mathrm{u}_{3} \mathrm{v}\right)\right]\right\}=\alpha_{5} \mathrm{u}_{1}\left(\mathrm{u}_{2} \mathrm{v}\right)$, por (1.14). Desse modo, $\alpha_{5}=\alpha_{7}=$ $=0$ e $\operatorname{dim} U \geq 7$. Mostremos que $\left\{\mathrm{u}_{1}\left(\mathrm{u}_{2} \mathrm{v}\right), \mathrm{u}_{1}\left(\mathrm{u}_{3} \mathrm{v}\right), \mathrm{u}_{2}\left(\mathrm{u}_{3} \mathrm{v}\right), \mathrm{v}\right\} \subseteq$ $\subseteq \mathrm{V}$ é livre. Para isso, suponhamos $\beta_{1} \mathrm{u}_{1}\left(\mathrm{u}_{2} \mathrm{v}\right)+\beta_{2} \mathrm{u}_{1}\left(\mathrm{u}_{3} \mathrm{v}\right)+\beta_{3} \mathrm{u}_{2}\left(\mathrm{u}_{3} \mathrm{v}\right)+$ $+\beta_{4} \mathrm{v}=0$. Então, multiplicando por $\mathrm{u}_{1}$, segue que $\beta_{1} \mathrm{u}_{1}\left[\mathrm{u}_{1}\left(\mathrm{u}_{2} \mathrm{v}\right)\right]+$ $+\beta_{2} \mathrm{u}_{1}\left[\mathrm{u}_{1}\left(\mathrm{u}_{3} \mathrm{v}\right)\right]+\beta_{3} \mathrm{u}_{1}\left[\mathrm{u}_{2}\left(\mathrm{u}_{3} \mathrm{v}\right)\right]+\beta_{4} \mathrm{u}_{1} \mathrm{v}=\beta_{3} \mathrm{u}_{1}\left[\mathrm{u}_{2}\left(\mathrm{u}_{3} \mathrm{v}\right)\right]+\beta_{4} \mathrm{u}_{1} \mathrm{v}=0$. pois por $(4.1) \mathrm{u}_{1}\left[\mathrm{u}_{1}\left(\mathrm{u}_{i} \mathrm{v}\right)\right] \in \mathrm{U}^{2}(\mathrm{UV})=0$ e portanto, de $\mathrm{J}\left(\mathrm{u}_{1}, \mathrm{u}_{2}, \mathrm{u}_{3} \mathrm{v}\right)=0$ e de $U^{2}(U V)=0$, obtemos $-\beta_{3} \mathrm{u}_{2}\left[\mathrm{u}_{1}\left(\mathrm{u}_{3} \mathrm{v}\right)\right]+\beta_{4} \mathrm{u}_{1} \mathrm{v}=0$. Multiplicando por $\mathrm{u}_{2}$, segue que $0=-\beta_{3} \mathrm{u}_{2}\left\{\mathrm{u}_{2}\left[\mathrm{u}_{1}\left(\mathrm{u}_{3} \mathrm{v}\right)\right]\right\}+\beta_{4} \mathrm{u}_{2}\left(\mathrm{u}_{1} \mathrm{v}\right)=\beta_{4} \mathrm{u}_{2}\left(\mathrm{u}_{1} \mathrm{v}\right)=0($ de (1.14)) e portanto $\beta_{4}=0$, o que implica em $\beta_{3}=0$. Logo, $\beta_{1} \mathrm{u}_{1}\left(\mathrm{u}_{2} \mathrm{v}\right)+$ $+\beta_{2} \mathrm{u}_{1}\left(\mathrm{u}_{3} \mathrm{v}\right)$. Multiplicando novamente por $\mathrm{u}_{2}$, temos $0=\beta_{1} \mathrm{u}_{2}\left[\mathrm{u}_{1}\left(\mathrm{u}_{2} \mathrm{v}\right)\right]+$ $+\beta_{2} \mathrm{u}_{2}\left[\mathrm{u}_{1}\left(\mathrm{u}_{3} \mathrm{v}\right)\right]=\beta_{2} \mathrm{u}_{2}\left[\mathrm{u}_{1}\left(\mathrm{u}_{3} \mathrm{v}\right)\right]$, sendo que esta última igualdade é consequência da identidade de Jacobi aplicada aos elementos $\mathrm{u}_{1}, \mathrm{u}_{2}, \mathrm{u}_{2} \mathrm{v}$ e de $\mathrm{U}^{2}(\mathrm{UV})=0$. Portanto, $\beta_{2}$ e $\beta_{1}$ são ambos nulos e $\operatorname{assim} \operatorname{dim} \mathrm{V} \geq 4 \mathrm{e}$ $\operatorname{dim} \mathrm{A} \geq 12$.

Exemplo 11: O Exemplo 10 confirma o resultado da parte (i) do Lema anterior. Quanto à parte (ii), a álgebra $\mathrm{A}$ com base $\left\{\mathrm{e}, \mathrm{u}_{1}, \mathrm{u}_{2}, \mathrm{u}_{3}, \mathrm{u}_{4}, \mathrm{u}_{5}\right.$, $\left.\mathrm{u}_{6}, \mathrm{u}_{7}, \mathrm{v}_{1}, \mathrm{v}_{2}, \mathrm{v}_{3}, \mathrm{v}_{4}\right\}$ e tábua de multiplicação

$$
\left\{\begin{array}{l}
\mathrm{e}^{2}=\mathrm{e} ; 2 \mathrm{eu}_{\mathrm{i}}=\mathrm{u}_{\mathrm{i}}(\mathrm{i}=1, \ldots, 7) ; \quad \mathrm{ev}_{\mathrm{i}}=0(\mathrm{i}=1, \ldots, 4) \\
\mathrm{u}_{1} \mathrm{u}_{5}=-\mathrm{u}_{2} \mathrm{u}_{4}=-\mathrm{v}_{2} ; \quad \mathrm{u}_{1} \mathrm{u}_{6}=-\mathrm{u}_{3} \mathrm{u}_{4}=-\mathrm{v}_{3} ; \mathrm{u}_{2} \mathrm{u}_{6}=-\mathrm{u}_{3} \mathrm{u}_{5}=\mathrm{v}_{1} \\
\mathrm{u}_{1} \mathrm{v}_{1}=\mathrm{u}_{2} \mathrm{v}_{3}=-\mathrm{u}_{3} \mathrm{v}_{2}=\mathrm{u}_{7} ; \mathrm{u}_{1} \mathrm{v}_{4}=\mathrm{u}_{4}: \mathrm{u}_{2} \mathrm{v}_{4}=\mathrm{u}_{5} ; \quad \mathrm{u}_{3} \mathrm{v}_{4}=\mathrm{u}_{6} \\
\text { os outros produtos são nulos }
\end{array}\right.
$$


satisfaz $\mathrm{x}^{3}=\omega(\mathrm{x}) \mathrm{x}^{2}, \operatorname{com} \mathrm{U}[\mathrm{U}(\mathrm{UV})] \neq 0\left(\right.$ pois $\mathrm{u}_{1}\left[\mathrm{u}_{2}\left(\mathrm{u}_{3} \mathrm{v}_{4}\right)\right]=\mathrm{u}_{1}\left(\mathrm{u}_{2} \mathrm{u}_{6}\right)=$ $=\mathrm{u}_{1} \mathrm{v}_{1}=\mathrm{u}_{7}$ ), $\mathrm{U}^{3} \mathrm{~V}=0$ (pois $\mathrm{U}^{3}=\mathrm{Fu}_{7}$ e $\mathrm{u}_{7} \mathrm{~V}=0$ ) e dimensão 12.

Lema 4.12 Se o P-monômio $U[(U V) V]$ é não nulo, então $r \geq 8, s \geq$ $\geq 3$ e portanto $\operatorname{dim} A \geq 12$.

Prova: Existem $u_{1}, u_{2} \in U$ e $v_{1}, v_{2} \in V$ tais que $u_{2}\left[\left(u_{1} v_{1}\right) v_{2}\right]=$ $=-\left(\mathrm{u}_{1} \mathrm{v}_{1}\right)\left(\mathrm{u}_{2} \mathrm{v}_{2}\right) \neq 0$. Afirmo que $\left\{\mathrm{u}_{1}, \mathrm{u}_{2}, \mathrm{u}_{1} \mathrm{v}_{1}, \mathrm{u}_{1} \mathrm{v}_{2}, \mathrm{u}_{2} \mathrm{v}_{1}, \mathrm{u}_{2} \mathrm{v}_{2},\left(\mathrm{u}_{1} \mathrm{v}_{1}\right) \mathrm{v}_{2}\right.$, $\left.\left(u_{2} v_{2}\right) v_{1}\right\}$ é livre. De fato, se tivermos $\alpha_{1} u_{1}+\alpha_{2} u_{2}+\alpha_{3} u_{1} v_{1}+\alpha_{4} u_{1} v_{2}+$ $+\alpha_{5} \mathrm{u}_{2} \mathrm{v}_{1}+\alpha_{6} \mathrm{u}_{2} \mathrm{v}_{2}+\alpha_{7}\left(\mathrm{u}_{1} \mathrm{v}_{1}\right) \mathrm{v}_{2}+\alpha_{8}\left(\mathrm{u}_{2} \mathrm{v}_{2}\right) \mathrm{v}_{1}=0$ então multiplicando por $\left(\mathrm{u}_{2} \mathrm{v}_{2}\right) \mathrm{v}_{1}$ e usando as identidades (4.5) e (4.9), (4.10), (4.3) e (4.10), $(4.10),(1.14),(4.3)$ e $(4.10),(1.17)$, temos $\alpha_{1} \mathrm{u}_{1}\left[\left(\mathrm{u}_{2} \mathrm{v}_{2}\right) \mathrm{v}_{1}\right]=0$, o que implica em $\alpha_{1}=0$. Logo $\alpha_{2} \mathrm{u}_{2}+\alpha_{3} \mathrm{u}_{1} \mathrm{v}_{1}+\alpha_{4} \mathrm{u}_{1} \mathrm{v}_{2}+\alpha_{5} \mathrm{u}_{2} \mathrm{v}_{1}+\alpha_{6} \mathrm{u}_{2} \mathrm{v}_{2}+$ $+\alpha_{7}\left(\mathrm{u}_{1} \mathrm{v}_{1}\right) \mathrm{v}_{2}+\alpha_{8}\left(\mathrm{u}_{2} \mathrm{v}_{2}\right) \mathrm{v}_{1}=0$. Daí, multiplicando agora por $\mathrm{v}_{1}$ e de (1.15) e (4.3), vem que $\alpha_{2} \mathrm{u}_{2} \mathrm{v}_{1}+\alpha_{4}\left(\mathrm{u}_{1} \mathrm{v}_{2}\right) \mathrm{v}_{1}+\alpha_{6}\left(\mathrm{u}_{2} \mathrm{v}_{2}\right) \mathrm{v}_{1}=0$ e portanto $\alpha_{2}\left(\mathrm{u}_{2} \mathrm{v}_{1}\right) \mathrm{v}_{2}=0$ (novamente de (1.15) e (4.3)); ou seja, $\alpha_{2}=0$ e também $\alpha_{4}\left(\mathrm{u}_{1} \mathrm{v}_{2}\right) \mathrm{v}_{1}+\alpha_{6}\left(\mathrm{u}_{2} \mathrm{v}_{2}\right) \mathrm{v}_{1}=0$. Logo, multiplicando por $\mathrm{u}_{1}$ e utilizando (4.5) e (4.9), obtemos $\alpha_{6} \mathrm{u}_{1}\left[\left(\mathrm{u}_{2} \mathrm{v}_{2}\right) \mathrm{v}_{1}\right]=0$; isto é, $\alpha_{6}$ e $\alpha_{4}$ são ambos nulos. Note que aqui ficou mostrado que $\left\{u_{2} v_{1},\left(u_{1} v_{2}\right) v_{1},\left(u_{2} v_{2}\right) v_{1}\right\}$ é livre. Ficamos então com $\alpha_{3} \mathrm{u}_{1} \mathrm{v}_{1}+\alpha_{5} \mathrm{u}_{2} \mathrm{v}_{1}+\alpha_{7}\left(\mathrm{u}_{1} \mathrm{v}_{1}\right) \mathrm{v}_{2}+\alpha_{8}\left(\mathrm{u}_{2} \mathrm{v}_{2}\right) \mathrm{v}_{1}=0$ e portanto (multiplicando por $\left.\mathrm{u}_{2} \mathrm{v}_{2}\right), \alpha_{3}\left(\mathrm{u}_{1} \mathrm{v}_{1}\right)\left(\mathrm{u}_{2} \mathrm{v}_{2}\right)=0$ (por (4.9), (4.10) e (1.14)). Isto implica em $\alpha_{3}=0$ e $0=\alpha_{5} \mathrm{u}_{2} \mathrm{v}_{1}+\alpha_{7}\left(\mathrm{u}_{1} \mathrm{v}_{1}\right) \mathrm{v}_{2}+\alpha_{8}\left(\mathrm{u}_{2} \mathrm{v}_{2}\right) \mathrm{v}_{1}=$ $=\alpha_{5} \mathrm{u}_{2} \mathrm{v}_{1}-\alpha_{7}\left(\mathrm{u}_{1} \mathrm{v}_{2}\right) \mathrm{v}_{1}+\alpha_{8}\left(\mathrm{u}_{2} \mathrm{v}_{2}\right) \mathrm{v}_{1}$, sendo esta última igualdade consequência de (4.3). Logo, $\alpha_{5}=\alpha_{7}=\alpha_{8}=0$ pois, como observamos acima, $\left\{u_{2} v_{1},\left(u_{1} v_{2}\right) v_{1},\left(u_{2} v_{2}\right) v_{1}\right\}$ é livre. Segue que $r \geq 8$. Além disso, temos que $\mathrm{s} \geq 3$ pois $\left\{\mathrm{v}_{1}, \mathrm{v}_{2},\left(\mathrm{u}_{1} \mathrm{v}_{1}\right)\left(\mathrm{u}_{2} \mathrm{v}_{2}\right)\right\}$ é livre, uma vez que se $\beta_{1} \mathrm{v}_{1}+$ $+\beta_{2} \mathrm{v}_{2}+\beta_{3}\left(\mathrm{u}_{1} \mathrm{v}_{1}\right)\left(\mathrm{u}_{2} \mathrm{v}_{2}\right)=0$ então, multiplicando por $\mathrm{u}_{1}$, segue que $\beta_{1} \mathrm{u}_{1} \mathrm{v}_{1}+\beta_{2} \mathrm{u}_{1} \mathrm{v}_{2}+\beta_{3} \mathrm{u}_{1}\left[\left(\mathrm{u}_{1} \mathrm{v}_{1}\right)\left(\mathrm{u}_{2} \mathrm{v}_{2}\right)\right]=0$ e, multiplicando agora por $\mathrm{u}_{2} \mathrm{v}_{2}$ e usando as identidades (4.10) e (4.15), temos $\beta_{1}\left(\mathrm{u}_{1} \mathrm{v}_{1}\right)\left(\mathrm{u}_{2} \mathrm{v}_{2}\right)=0$; isto é, $\beta_{1}=0$ e $\beta_{2} \mathrm{v}_{2}+\beta_{3}\left(\mathrm{u}_{1} \mathrm{v}_{1}\right)\left(\mathrm{u}_{2} \mathrm{v}_{2}\right)=0$. Finalmente, multiplicando por $\mathrm{u}_{1} \mathrm{v}_{1}$, de $(4.16)$ vem que $\beta_{2}\left(\mathrm{u}_{1} \mathrm{v}_{1}\right) \mathrm{v}_{2}=0$. Portanto, $\beta_{2}$ e $\beta_{3}$ são ambos nulos. Ou seja, $\operatorname{dim} \mathrm{V} \geq 4$ e $\operatorname{dim} \mathrm{A} \geq 12$. 
Exemplo 12: A álgebra A com base $\left\{e, u_{1}, u_{2}, u_{3}, u_{4}, u_{5}, u_{6}, u_{\overline{7}}, u_{8}, v_{1}\right.$. $\left.\mathrm{v}_{2}, \mathrm{v}_{3}\right\}$ e tábua de multiplicação

$$
\left\{\begin{array}{l}
\mathrm{e}^{2}=\mathrm{e} ; 2 \mathrm{eu}_{\mathrm{i}}=\mathrm{u}_{\mathrm{i}}(\mathrm{i}=1, \ldots 8) ; \quad \mathrm{v}_{\mathrm{i}}=0 \quad(\mathrm{i}=1,2,3) \\
\mathrm{u}_{1} \mathrm{u}_{4}=\mathrm{u}_{1} \mathrm{u}_{5}=\mathrm{u}_{3} \mathrm{u}_{4}=\mathrm{v}_{3} \\
\mathrm{u}_{1} \mathrm{u}_{7}=\mathrm{u}_{2} \mathrm{u}_{3}=\mathrm{u}_{2} \mathrm{u}_{6}=\mathrm{u}_{2} \mathrm{u}_{8}=\mathrm{u}_{5} \mathrm{u}_{6}=-\mathrm{v}_{3} \\
\mathrm{u}_{1} \mathrm{v}_{1}=\mathrm{u}_{3} ; \mathrm{u}_{1} \mathrm{v}_{2}=\mathrm{u}_{6} \\
\mathrm{u}_{2} \mathrm{v}_{1}=\mathrm{u}_{5} ; \quad \mathrm{u}_{2} \mathrm{v}_{2}=\mathrm{u}_{4} \\
\mathrm{u}_{4} \mathrm{v}_{1}=-\mathrm{u}_{5} \mathrm{v}_{2}=\mathrm{u}_{7} \\
\mathrm{u}_{3} \mathrm{v}_{2}=-\mathrm{u}_{6} \mathrm{v}_{1}=\mathrm{u}_{8} \\
\text { os outros produtos são nulos }
\end{array}\right.
$$

satisfaz $\mathrm{x}^{3}=\omega(\mathrm{x}) \mathrm{x}^{2}$, com $\mathrm{U}[(\mathrm{UV}) \mathrm{V}] \neq 0\left(\right.$ pois $\mathrm{u}_{2}\left[\left(\mathrm{u}_{1} \mathrm{v}_{2}\right) \mathrm{v}_{1}\right]=\mathrm{u}_{2}\left(\mathrm{u}_{6} \mathrm{v}_{1}\right)=$ $\left.=-\mathrm{u}_{2} \mathrm{u}_{8}=\mathrm{v}_{3}\right)$ e dimensão 12 .

Antes de passarmos ao próximo resultado, faremos três observações que serão úteis à sua demonstração. Para isso, suponhamos que na tálgebra A fixada exista um elemento não nulo $\mathrm{u}_{2}\left[\left(\mathrm{u}_{1} \mathrm{v}_{1}\right) \mathrm{v}_{2}\right]$ tal que $\mathrm{u}_{1}^{2} \notin$ $\notin \operatorname{ann}\left\{\left(\mathrm{u}_{2} \mathrm{v}_{2}\right) \mathrm{v}_{1}\right\}$.

Observação 1: Afirmamos que, nas condições acima, o subconjunto $\left\{\mathrm{u}_{1}^{2}\left(\mathrm{u}_{2} \mathrm{v}_{2}\right), \mathrm{u}_{1}^{2}\left[\left(\mathrm{u}_{2} \mathrm{v}_{2}\right) \mathrm{v}_{1}\right]\right\}$ de $\mathrm{U}$ é livre. De fato, notemos, inicialmente. que $0 \neq \mathrm{u}_{1}^{2}\left[\left(\mathrm{u}_{2} \mathrm{v}_{2}\right) \mathrm{v}_{1}\right]=-\left[\left(\mathrm{u}_{2} \mathrm{v}_{2}\right) \mathrm{u}_{1}^{2}\right] \mathrm{v}_{1}$ (por (4.3)) e portanto $\left(\mathrm{u}_{2} \mathrm{v}_{2}\right) \mathrm{u}_{1}^{2} \neq$ $\neq 0$. Suponhamos, agora, que $\alpha \mathrm{u}_{1}^{2}\left(\mathrm{u}_{2} \mathrm{v}_{2}\right)+\beta \mathrm{u}_{1}^{2}\left[\left(\mathrm{u}_{2} \mathrm{v}_{2}\right) \mathrm{v}_{1}\right]=0$. Então, multiplicando por $\mathrm{v}_{1}$ e de $(4.3)$ e $(1.15)$, obtemos $0=\alpha\left[\mathrm{u}_{1}^{2}\left(\mathrm{u}_{2} \mathrm{v}_{2}\right)\right] \mathrm{v}_{1}=$ $=-\alpha u_{1}^{2}\left[\left(u_{2} v_{2}\right) v_{1}\right]$. Logo $\alpha=\beta=0$ e o conjunto dado é livre.

Observação 2: Nas condições acima, o subconjunto $\left\{\mathrm{u}_{1}^{2} \mathrm{u}_{2}, \mathrm{u}_{1}^{2}\left(\mathrm{u}_{2} \mathrm{v}_{1}\right)\right.$, $\left.u_{1}^{2}\left(u_{2} v_{2}\right), u_{1}^{2}\left[\left(u_{2} v_{2}\right) v_{1}\right]\right\}$ de $U$ é livre. De fato, inicialmente notemos que, de (4.3), $0 \neq u_{1}^{2}\left(u_{2} v_{2}\right)=-\left(u_{1}^{2} u_{2}\right) v_{2}$ e portanto $u_{1}^{2} u_{2} \neq 0$. Além disso, ainda de (4.3), $0 \neq \mathrm{u}_{1}^{2}\left[\left(\mathrm{u}_{2} \mathrm{v}_{2}\right) \mathrm{v}_{1}\right]=-\left[\mathrm{u}_{1}^{2}\left(\mathrm{u}_{2} \mathrm{v}_{2}\right)\right] \mathrm{v}_{1}=\left[\left(\mathrm{u}_{1}^{2} \mathrm{u}_{2}\right) \mathrm{v}_{2}\right] \mathrm{v}_{1}=-\left[\left(\mathrm{u}_{1}^{2} \mathrm{u}_{2}\right) \mathrm{v}_{1}\right] \mathrm{v}_{2} \mathrm{e}$ portanto $\mathrm{u}_{1}^{2}\left(\mathrm{u}_{2} \mathrm{v}_{1}\right) \neq 0$. Suponhamos, agora, que $\lambda_{1} \mathrm{u}_{1}^{2} \mathrm{u}_{2}+\lambda_{2} \mathrm{u}_{1}^{2}\left(\mathrm{u}_{2} \mathrm{v}_{1}\right)+$ 
$+\lambda_{3} \mathrm{u}_{1}^{2}\left(\mathrm{u}_{2} \mathrm{v}_{2}\right)+\lambda_{4} \mathrm{u}_{1}^{2}\left[\left(\mathrm{u}_{2} \mathrm{v}_{2}\right) \mathrm{v}_{1}\right]=0$. Multiplicando por $\mathrm{v}_{2}$, de (4.3) e (1.15) obtemos $0=\lambda_{1}\left(\mathrm{u}_{1}^{2} \mathrm{u}_{2}\right) \mathrm{v}_{2}+\lambda_{2}\left[\mathrm{u}_{1}^{2}\left(\mathrm{u}_{2} \mathrm{v}_{1}\right)\right] \mathrm{v}_{2}=-\lambda_{1} \mathrm{u}_{1}^{2}\left(\mathrm{u}_{2} \mathrm{v}_{2}\right)-\lambda_{2} \mathrm{u}_{1}^{2}\left[\left(\mathrm{u}_{2} \mathrm{v}_{1}\right) \mathrm{v}_{2}\right]=$ $=-\lambda_{1} u_{1}^{2}\left(u_{2} v_{2}\right)+\lambda_{2} u_{1}^{2}\left[\left(u_{2} v_{2}\right) v_{1}\right]$ e assim, da observação 1 , segue que $\lambda_{1}=$ $=\lambda_{2}=0$. Logo, $\lambda_{3} \mathrm{u}_{1}^{2}\left(\mathrm{u}_{2} \mathrm{v}_{2}\right)+\lambda_{4} \mathrm{u}_{1}^{2}\left[\left(\mathrm{u}_{2} \mathrm{v}_{2}\right) \mathrm{v}_{1}\right]=0$ e novamente pela observação 1 , obtemos $\lambda_{3}=\lambda_{4}=0$. Ou seja, o conjunto dado é livre.

Observação 3: Mostremos que, nas condições acima, o subconjunto $\left\{\left(\mathrm{u}_{1} \mathrm{v}_{1}\right) \mathrm{v}_{2}, \mathrm{u}_{1}^{2}\left[\left(\mathrm{u}_{2} \mathrm{v}_{2}\right) \mathrm{v}_{1}\right]\right\}$ de U é livre. Para isso, suponhamos $\alpha\left(\mathrm{u}_{1} \mathrm{v}_{1}\right) \mathrm{v}_{2}+$ $+\beta \mathrm{u}_{1}^{2}\left[\left(\mathrm{u}_{2} \mathrm{v}_{2}\right) \mathrm{v}_{1}\right]=0$, multiplicando por $\mathrm{u}_{2}$, temos $0=\alpha \mathrm{u}_{2}\left[\left(\mathrm{u}_{1} \mathrm{v}_{1}\right) \mathrm{v}_{2}\right]+$ $+\beta \mathrm{u}_{2}\left\{\mathrm{u}_{1}^{2}\left[\left(\mathrm{u}_{2} \mathrm{v}_{2}\right) \mathrm{v}_{1}\right]\right\}=\alpha \mathrm{u}_{1}\left[\left(\mathrm{u}_{2} \mathrm{v}_{2}\right) \mathrm{v}_{1}\right]-\beta\left(\mathrm{u}_{1}^{2} \mathrm{u}_{2}\right)\left[\left(\mathrm{u}_{2} \mathrm{v}_{2}\right) \mathrm{v}_{1}\right]$ (por (4.5) e (4.7)). Finalmente, multiplicando agora por $2 \mathrm{u}_{1}$, de (4.19) e (4.1) obtemos $0=$ $=2 \alpha \mathrm{u}_{1}\left\{\mathrm{u}_{1}\left[\left(\mathrm{u}_{2} \mathrm{v}_{2}\right) \mathrm{v}_{1}\right]\right\}=-\alpha \mathrm{u}_{1}^{2}\left[\left(\mathrm{u}_{2} \mathrm{v}_{2}\right) \mathrm{v}_{1}\right]$ e portanto $\alpha=\beta=0$, o que mostra que o conjunto dado é livre.

Corolário 4.12.1 Nas condições do Lema 4.12, se o monômio de Peirce $U[(U V) V]$ admite um gerador aditivo $u_{2}\left[\left(u_{1} v_{1}\right) v_{2}\right]$ tal que $u_{1}^{2} \notin$ $\notin$ ann $\left\{\left(u_{2} v_{2}\right) v_{1}\right\}$, então $r \geq 12$ e $s \geq 7$ e portanto dim. $A \geq 20$.

Prova: Comecemos mostrando que o subconjunto $\left\{\mathrm{u}_{1}, \mathrm{u}_{2}, \mathrm{u}_{1} \mathrm{v}_{1}, \mathrm{u}_{1} \mathrm{v}_{2}\right.$, $\left.\mathrm{u}_{2} \mathrm{v}_{1}, \mathrm{u}_{2} \mathrm{v}_{2},\left(\mathrm{u}_{1} \mathrm{v}_{1}\right) \mathrm{v}_{2},\left(\mathrm{u}_{2} \mathrm{v}_{2}\right) \mathrm{v}_{1}, \mathrm{u}_{1}^{2} \mathrm{u}_{2}, \mathrm{u}_{1}^{2}\left(\mathrm{u}_{2} \mathrm{v}_{1}\right), \mathrm{u}_{1}^{2}\left(\mathrm{u}_{2} \mathrm{v}_{2}\right), \mathrm{u}_{1}^{2}\left[\left(\mathrm{u}_{2} \mathrm{v}_{2}\right) \mathrm{v}_{1}\right]\right\}$ de U é livre. Para isso, suponhamos $\alpha_{1} \mathrm{u}_{1}+\alpha_{2} \mathrm{u}_{2}+\alpha_{3} \mathrm{u}_{1} \mathrm{v}_{1}+\alpha_{4} \mathrm{u}_{1} \mathrm{v}_{2}+$ $+\alpha_{5} \mathrm{u}_{2} \mathrm{v}_{1}+\alpha_{6} \mathrm{u}_{2} \mathrm{v}_{2}+\alpha_{7}\left(\mathrm{u}_{1} \mathrm{v}_{1}\right) \mathrm{v}_{2}+\alpha_{8}\left(\mathrm{u}_{2} \mathrm{v}_{2}\right) \mathrm{v}_{1}+\alpha_{9} \mathrm{u}_{1}^{2} \mathrm{u}_{2}+\alpha_{10} \mathrm{u}_{1}^{2}\left(\mathrm{u}_{2} \mathrm{v}_{1}\right)+$ $+\alpha_{11} \mathrm{u}_{1}^{2}\left(\mathrm{u}_{2} \mathrm{v}_{2}\right)+\alpha_{12} \mathrm{u}_{1}^{2}\left[\left(\mathrm{u}_{2} \mathrm{v}_{2}\right) \mathrm{v}_{1}\right]=0$. Multiplicando por $\mathrm{u}_{1}^{2}$, obtemos $\alpha_{2} \mathrm{u}_{1}^{2} \mathrm{u}_{2}+\alpha_{5} \mathrm{u}_{1}^{2}\left(\mathrm{u}_{2} \mathrm{v}_{1}\right)+\alpha_{6} \mathrm{u}_{1}^{2}\left(\mathrm{u}_{2} \mathrm{v}_{2}\right)+\alpha_{8} \mathrm{u}_{1}^{2}\left[\left(\mathrm{u}_{2} \mathrm{v}_{2}\right) \mathrm{v}_{1}\right]=0$ (de (1.15), (1.16) e (4.17)) e portanto, pela observação 2 acima, $\alpha_{2}=\alpha_{5}=\alpha_{6}=\alpha_{8}=$ $=0$. Dessa forma, $\alpha_{1} \mathrm{u}_{1}+\alpha_{3} \mathrm{u}_{1} \mathrm{v}_{1}+\alpha_{4} \mathrm{u}_{1} \mathrm{v}_{2}+\alpha_{7}\left(\mathrm{u}_{1} \mathrm{v}_{1}\right) \mathrm{v}_{2}+\alpha_{9} \mathrm{u}_{1}^{2} \mathrm{u}_{2}+$ $+\alpha_{10} \mathrm{u}_{1}^{2}\left(\mathrm{u}_{2} \mathrm{v}_{1}\right)+\alpha_{11} \mathrm{u}_{1}^{2}\left(\mathrm{u}_{2} \mathrm{v}_{2}\right)+\alpha_{12} \mathrm{u}_{1}^{2}\left[\left(\mathrm{u}_{2} \mathrm{v}_{2}\right) \mathrm{v}_{1}\right]=0$. Multiplicando agora por $\mathrm{u}_{1}$, de (1.14), (4.11) e (1.18) segue que $\alpha_{1} \mathrm{u}_{1}^{2}=0$; ou seja, $\alpha_{1}=$ $=0$ e portanto $\alpha_{3} \mathrm{u}_{1} \mathrm{v}_{1}+\alpha_{4} \mathrm{u}_{1} \mathrm{v}_{2}+\alpha_{7}\left(\mathrm{u}_{1} \mathrm{v}_{1}\right) \mathrm{v}_{2}+\alpha_{9} \mathrm{u}_{1}^{2} \mathrm{u}_{2}+\alpha_{10} \mathrm{u}_{1}^{2}\left(\mathrm{u}_{2} \mathrm{v}_{1}\right)+$ $+\alpha_{11} \mathrm{u}_{1}^{2}\left(\mathrm{u}_{2} \mathrm{v}_{2}\right)+\alpha_{12} \mathrm{u}_{1}^{2}\left[\left(\mathrm{u}_{2} \mathrm{v}_{2}\right) \mathrm{v}_{1}\right]=0$. Multiplicando por $\mathrm{u}_{2} \mathrm{v}_{2}$, de (4.9). (4.10), (1.14), obtemos $\alpha_{3}\left(\mathrm{u}_{1} \mathrm{v}_{1}\right)\left(\mathrm{u}_{2} \mathrm{v}_{2}\right)+\alpha_{10}\left[\mathrm{u}_{1}^{2}\left(\mathrm{u}_{2} \mathrm{v}_{1}\right)\right]\left(\mathrm{u}_{2} \mathrm{v}_{2}\right)=0$. Decorre daí, multiplicando por $2 \mathrm{u}_{1}$, que $0=2 \alpha_{3} \mathrm{u}_{1}\left[\left(\mathrm{u}_{1} \mathrm{v}_{1}\right)\left(\mathrm{u}_{2} \mathrm{v}_{2}\right)\right]=\alpha_{3} \mathrm{u}_{1}^{2}\left[\left(\mathrm{u}_{2} \mathrm{v}_{2}\right) \mathrm{v}_{1}\right]$ 
(por (4.8) e (4.18)) e portanto $\alpha_{3}=0$. Logo, $\alpha_{4} \mathrm{u}_{1} \mathrm{v}_{2}+\alpha_{7}\left(\mathrm{u}_{1} \mathrm{v}_{1}\right) \mathrm{v}_{2}+$ $+\alpha_{9} \mathrm{u}_{1}^{2} \mathrm{u}_{2}+\alpha_{10} \mathrm{u}_{1}^{2}\left(\mathrm{u}_{2} \mathrm{v}_{1}\right)+\alpha_{11} \mathrm{u}_{1}^{2}\left(\mathrm{u}_{2} \mathrm{v}_{2}\right)+\alpha_{12} \mathrm{u}_{1}^{2}\left[\left(\mathrm{u}_{2} \mathrm{v}_{2}\right) \mathrm{v}_{1}\right]=0$ e portanto, multiplicando por $\mathrm{v}_{2}$, de (1.15) e (4.3) vem que $0=\alpha_{9}\left(\mathrm{u}_{1}^{2} \mathrm{u}_{2}\right) \mathrm{v}_{2}+$ $+\alpha_{10}\left[u_{1}^{2}\left(u_{2} v_{1}\right)\right] v_{2}$. Dessa forma, multiplicando agora por $v_{1}$, obtemos $\alpha_{9}\left[\left(u_{1}^{2} u_{2}\right) v_{2}\right] v_{1}=0$ (por (4.3) e (1.15)). Logo, de $\left[\left(u_{1}^{2} u_{2}\right) v_{2}\right] v_{j} \neq 0$ (vide Observação 2), segue que $\alpha_{9}=\alpha_{10}=0$, o que implica em $\alpha_{4} u_{1} v_{2}+$ $+\alpha_{7}\left(\mathrm{u}_{1} \mathrm{v}_{1}\right) \mathrm{v}_{2}+\alpha_{11} \mathrm{u}_{1}^{2}\left(\mathrm{u}_{2} \mathrm{v}_{2}\right)+\alpha_{12} \mathrm{u}_{1}^{2}\left[\left(\mathrm{u}_{2} \mathrm{v}_{2}\right) \mathrm{v}_{1}\right]=0$. Multiplicando por $\mathrm{v}_{1}$, de (4.3) e (1.15) ficamos com $0=\alpha_{4}\left(\mathrm{u}_{1} \mathrm{v}_{2}\right) \mathrm{v}_{1}+\alpha_{11}\left[\mathrm{u}_{1}^{2}\left(\mathrm{u}_{2} \mathrm{v}_{2}\right)\right] \mathrm{v}_{1}=$ $=-\alpha_{4}\left(\mathrm{u}_{1} \mathrm{v}_{1}\right) \mathrm{v}_{2}-\alpha_{11} \mathrm{u}_{1}^{2}\left[\left(\mathrm{u}_{2} \mathrm{v}_{2}\right) \mathrm{v}_{1}\right]$ e, pela observação $3, \alpha_{4}=\alpha_{11}=0$. Dessa forma, novamente pela observação $3, \alpha_{7}=\alpha_{12}=0$, o que completa a prova de que $\operatorname{dim} U \geq 12$. Afirmamos agora que $\left\{\mathrm{v}_{1}, \mathrm{v}_{2}, \mathrm{u}_{1}^{2}, \mathrm{u}_{1} \mathrm{u}_{2}, \mathrm{u}_{1}\left(\mathrm{u}_{2} \mathrm{v}_{2}\right)\right.$, $\left.\mathrm{u}_{2}\left(\mathrm{u}_{1} \mathrm{v}_{1}\right),\left(\mathrm{u}_{1} \mathrm{v}_{1}\right)\left(\mathrm{u}_{2} \mathrm{v}_{2}\right)\right\} \subseteq \mathrm{V}$ é livre. De fato, se $\beta_{1} \mathrm{v}_{1}+\beta_{2} \mathrm{v}_{2}+\beta_{3} \mathrm{u}_{1}^{2}+$ $+\beta_{4} \mathrm{u}_{1} \mathrm{u}_{2}+\beta_{5} \mathrm{u}_{1}\left(\mathrm{u}_{2} \mathrm{v}_{2}\right)+\beta_{6} \mathrm{u}_{2}\left(\mathrm{u}_{1} \mathrm{v}_{1}\right)+\beta_{7}\left(\mathrm{u}_{1} \mathrm{v}_{1}\right)\left(\mathrm{u}_{2} \mathrm{v}_{2}\right)=0$, então multiplicando por $\mathrm{u}_{2} \mathrm{v}_{2}$, de (1.15), (4.1), (1.17) e (4.16) segue que $\beta_{1}\left(\mathrm{u}_{2} \mathrm{v}_{2}\right) \mathrm{v}_{1}+$ $+\beta_{3} \mathrm{u}_{1}^{2}\left(\mathrm{u}_{2} \mathrm{v}_{2}\right)+\beta_{4}\left(\mathrm{u}_{1} \mathrm{u}_{2}\right)\left(\mathrm{u}_{2} \mathrm{v}_{2}\right)+\beta_{6}\left[\mathrm{u}_{2}\left(\mathrm{u}_{1} \mathrm{v}_{1}\right)\right]\left(\mathrm{u}_{2} \mathrm{v}_{2}\right)=0$ e assim (multiplicando por $\left.\mathrm{u}_{1}^{2}\right), \beta_{1} \mathrm{u}_{1}^{2}\left[\left(\mathrm{u}_{2} \mathrm{v}_{2}\right) \mathrm{v}_{1}\right]=0$ (por (1.15), (4.20) e (4.21)) e portanto $\beta_{1}=0$. Ficamos então com $\beta_{2} \mathrm{v}_{2}+\beta_{3} \mathrm{u}_{1}^{2}+\beta_{4} \mathrm{u}_{1} \mathrm{u}_{2}+\beta_{5} \mathrm{u}_{1}\left(\mathrm{u}_{2} \mathrm{v}_{2}\right)+$ $+\beta_{6} \mathrm{u}_{2}\left(\mathrm{u}_{1} \mathrm{v}_{1}\right)+\beta_{7}\left(\mathrm{u}_{1} \mathrm{v}_{1}\right)\left(\mathrm{u}_{2} \mathrm{v}_{2}\right)=0$, o que implica em $\beta_{2}\left(\mathrm{u}_{1} \mathrm{v}_{1}\right) \mathrm{v}_{2}+$ $+\beta_{4}\left(\mathrm{u}_{1} \mathrm{u}_{2}\right)\left(\mathrm{u}_{1} \mathrm{v}_{1}\right)+\beta_{5}\left[\mathrm{u}_{1}\left(\mathrm{u}_{2} \mathrm{v}_{2}\right)\right]\left(\mathrm{u}_{1} \mathrm{v}_{1}\right)=0$ (da multiplicação por $\mathrm{u}_{1} \mathrm{v}_{1} \mathrm{e}$ de (1.16), (4.1), (1.17) e (4.16)). Portanto, multiplicando por $\mathrm{u}_{2}$, obtemos $\beta_{2} \mathrm{u}_{2}\left[\left(\mathrm{u}_{1} \mathrm{v}_{1}\right) \mathrm{v}_{2}\right]+\beta_{5} \mathrm{u}_{2}\left\{\left[\mathrm{u}_{1}\left(\mathrm{u}_{2} \mathrm{v}_{2}\right)\right]\left(\mathrm{u}_{1} \mathrm{v}_{1}\right)\right\}=0$ (por (4.22)). Assim, multiplicando por $2 \mathrm{u}_{1}$ e usando as identidades (4.23), (4.5) e (4.1), segue que $2 \beta_{2} \mathrm{u}_{1}\left\{\mathrm{u}_{1}\left[\left(\mathrm{u}_{2} \mathrm{v}_{2}\right) \mathrm{v}_{1}\right]\right\}=-\beta_{2} \mathrm{u}_{1}^{2}\left[\left(\mathrm{u}_{2} \mathrm{v}_{2}\right) \mathrm{v}_{1}\right]=0$ e portanto $\beta_{2}=0$. Daí obte$\operatorname{mos} \beta_{3} \mathrm{u}_{1}^{2}+\beta_{4} \mathrm{u}_{1} \mathrm{u}_{2}+\beta_{5} \mathrm{u}_{1}\left(\mathrm{u}_{2} \mathrm{v}_{2}\right)+\beta_{6} \mathrm{u}_{2}\left(\mathrm{u}_{1} \mathrm{v}_{1}\right)+\beta_{7}\left(\mathrm{u}_{1} \mathrm{v}_{1}\right)\left(\mathrm{u}_{2} \mathrm{v}_{2}\right)=0$. Logo, se multiplicarmos por $2 \mathrm{u}_{1} \mathrm{v}_{1}$, usando as identidades (1.16), (4.1) e $(1.17),(4.16)$, teremos $0=2 \beta_{4}\left(\mathrm{u}_{1} \mathrm{u}_{2}\right)\left(\mathrm{u}_{1} \mathrm{v}_{1}\right)+2 \beta_{5}\left[\mathrm{u}_{1}\left(\mathrm{u}_{2} \mathrm{v}_{2}\right)\right]\left(\mathrm{u}_{1} \mathrm{v}_{1}\right)=$ $=-\beta_{4} \mathrm{u}_{1}^{2}\left(\mathrm{u}_{2} \mathrm{v}_{1}\right)-\beta_{5} \mathrm{u}_{1}^{2}\left[\left(\mathrm{u}_{2} \mathrm{v}_{2}\right) \mathrm{v}_{1}\right]$ (sendo esta última igualdade consequência de (4.2)). Como, pela observação $2,\left\{\mathrm{u}_{1}^{2}\left(\mathrm{u}_{2} \mathrm{v}_{1}\right), \mathrm{u}_{1}^{2}\left[\left(\mathrm{u}_{2} \mathrm{v}_{2}\right) \mathrm{v}_{1}\right]\right\}$ é livre, obte$\operatorname{mos} \beta_{4}=\beta_{5}=0$ e $\beta_{3} \mathrm{u}_{1}^{2}+\beta_{6} \mathrm{u}_{2}\left(\mathrm{u}_{1} \mathrm{v}_{1}\right)+\beta_{7}\left(\mathrm{u}_{1} \mathrm{v}_{1}\right)\left(\mathrm{u}_{2} \mathrm{v}_{2}\right)=0$. Finalmente, de uma nova multiplicação por $2 \mathrm{u}_{1}$, de (4.4). (4.5) e (4.1), respectivamente, temos $2 \beta_{6} \mathrm{u}_{1}\left[\mathrm{u}_{2}\left(\mathrm{u}_{1} \mathrm{v}_{1}\right)\right]+2 \beta_{7} \mathrm{u}_{1}\left[\left(\mathrm{u}_{1} \mathrm{v}_{1}\right)\left(\mathrm{u}_{2} \mathrm{v}_{2}\right)\right]=-2 \beta_{6} \mathrm{u}_{1}\left[\mathrm{u}_{1}\left(\mathrm{u}_{2} \mathrm{v}_{1}\right)\right]+$ $-2 \beta_{7} \mathrm{u}_{1}\left\{\mathrm{u}_{1}\left[\left(\mathrm{u}_{2} \mathrm{v}_{2}\right) \mathrm{v}_{1}\right]\right\}=\beta_{6} \mathrm{u}_{1}^{2}\left(\mathrm{u}_{2} \mathrm{v}_{1}\right)+\beta_{7} \mathrm{u}_{1}^{2}\left[\left(\mathrm{u}_{2} \mathrm{v}_{2}\right) \mathrm{v}_{1}\right]=0$ e como acima, $\beta_{6}=\beta_{7}=0$. Logo, $\beta_{3}=0$. Portanto, $\operatorname{dim} \mathrm{V} \geq 7$ e $\operatorname{dim} \mathrm{A} \geq 20$. 
Exemplo 13: A álgebra A com base $\left\{e, u_{1}, u_{2}, u_{3}, u_{4}, u_{5}, u_{6}, u_{7}, u_{8}, u_{9}\right.$. $\left.\mathrm{u}_{10}, \mathrm{u}_{11}, \mathrm{u}_{12}, \mathrm{v}_{1}, \mathrm{v}_{2}, \mathrm{v}_{3}, \mathrm{v}_{4}, \mathrm{v}_{5}, \mathrm{v}_{6}, \mathrm{v}_{7}\right\}$ e tábua de multiplicação

$$
\left\{\begin{array}{l}
\mathrm{e}^{2}=\mathrm{e} ; 2 \mathrm{eu}_{\mathrm{i}}=\mathrm{u}_{\mathrm{i}}(\mathrm{i}=1, \ldots, 12) ; \quad \mathrm{v}_{\mathrm{i}}=0(\mathrm{i}=1, \ldots, 7) \\
\mathrm{u}_{2}^{2}=\mathrm{v}_{3} ; \quad \mathrm{u}_{1} \mathrm{u}_{2}=\mathrm{v}_{4} ;-\mathrm{u}_{1} \mathrm{u}_{5}=\mathrm{u}_{2} \mathrm{u}_{3}=\mathrm{v}_{5} \\
\mathrm{u}_{1} \mathrm{u}_{4}=-\mathrm{u}_{2} \mathrm{u}_{6}=\mathrm{v}_{6} ; \quad \mathrm{u}_{1} \mathrm{u}_{7}=\mathrm{u}_{2} \mathrm{u}_{8}=-\mathrm{u}_{3} \mathrm{u}_{4}=\mathrm{u}_{5} \mathrm{u}_{6}=-\mathrm{v}_{7} \\
\mathrm{u}_{1} \mathrm{v}_{1}=\mathrm{u}_{3} ; \quad \mathrm{u}_{1} \mathrm{v}_{2}=\mathrm{u}_{6} ; \quad \mathrm{u}_{2} \mathrm{v}_{1}=\mathrm{u}_{5} ; \quad \mathrm{u}_{2} \mathrm{v}_{2}=\mathrm{u}_{4} \\
\mathrm{u}_{4} \mathrm{v}_{1}=-\mathrm{u}_{5} \mathrm{v}_{2}=\mathrm{u}_{7} ; \quad \mathrm{u}_{3} \mathrm{v}_{2}=-\mathrm{u}_{6} \mathrm{v}_{1}=\mathrm{u}_{8} \\
\mathrm{u}_{1} \mathrm{v}_{3}=-2 \mathrm{u}_{2} \mathrm{v}_{4}=\mathrm{u}_{9} ;-2 \mathrm{u}_{2} \mathrm{v}_{5}=\mathrm{u}_{3} \mathrm{v}_{3}=-2 \mathrm{u}_{5} \mathrm{v}_{4}=-\mathrm{u}_{9} \mathrm{v}_{1}=\mathrm{u}_{10} \\
2 \mathrm{u}_{2} \mathrm{v}_{6}=-2 \mathrm{u}_{4} \mathrm{v}_{4}=\mathrm{u}_{6} \mathrm{v}_{3}=-\mathrm{u}_{9} \mathrm{v}_{2}=\mathrm{u}_{11} \\
2 \mathrm{u}_{2} \mathrm{v}_{7}=-2 \mathrm{u}_{4} \mathrm{v}_{5}=-2 \mathrm{u}_{5} \mathrm{v}_{6}=2 \mathrm{u}_{7} \mathrm{v}_{4}=\mathrm{u}_{8} \mathrm{v}_{3}=-\mathrm{u}_{10} \mathrm{v}_{2}=\mathrm{u}_{11} \mathrm{v}_{1}=\mathrm{u}_{12} \\
\text { os outros produtos são nulos }
\end{array}\right.
$$

satisfaz $\mathrm{x}^{3}=\omega(\mathrm{x}) \mathrm{x}^{2}$, com $\mathrm{U}[(\mathrm{UV}) \mathrm{V}] \neq 0$ (uma vez que $\mathrm{u}_{2}\left[\left(\mathrm{u}_{1} \mathrm{v}_{2}\right) \mathrm{v}_{1}\right]=$ $\left.=\mathrm{u}_{2}\left(\mathrm{u}_{6} \mathrm{v}_{1}\right)=-\mathrm{u}_{2} \mathrm{u}_{8}=\mathrm{v}_{7}\right), \mathrm{u}_{2}^{2} \notin \operatorname{ann}\left\{\left(\mathrm{u}_{1} \mathrm{v}_{2}\right) \mathrm{v}_{1}\right\} \quad\left(\right.$ pois $\mathrm{u}_{2}^{2}\left[\left(\mathrm{u}_{1} \mathrm{v}_{2}\right) \mathrm{v}_{1}\right]=$ $\left.=\mathrm{v}_{3}\left(\mathrm{u}_{6} \mathrm{v}_{1}\right)=-\mathrm{v}_{3} \mathrm{u}_{8}=-\mathrm{u}_{12}\right)$ e dimensão 20 .

Lema 4.13 Se o P-monômio $[(U V) V] V$ é não nulo, então $r \geq 8$, $s \geq 3$ e portanto $\operatorname{dim} A \geq 12$.

Prova: Existem $\mathrm{u}_{1} \in \mathrm{U}$ e $\mathrm{v}_{1}, \mathrm{v}_{2}, \mathrm{v}_{3} \in \mathrm{V}$ tais que $\left[\left(\mathrm{u}_{1} \mathrm{v}_{1}\right) \mathrm{v}_{2}\right] \mathrm{v}_{3} \neq 0$. Note que $\left[\left(\mathrm{u}_{1} \mathrm{v}_{1}\right) \mathrm{v}_{2}\right] \mathrm{v}_{3}=-\left[\left(\mathrm{u}_{1} \mathrm{v}_{1}\right) \mathrm{v}_{3}\right] \mathrm{v}_{2}=\left[\left(\mathrm{u}_{1} \mathrm{v}_{3}\right) \mathrm{v}_{1}\right] \mathrm{v}_{2}=\left[\left(\mathrm{u}_{1} \mathrm{v}_{2}\right) \mathrm{v}_{3}\right] \mathrm{v}_{1}=$ $=-\left[\left(u_{1} v_{2}\right) v_{3}\right] v_{1}$, sendo todas igualdades consequência de (4.3). Segue então que $\left(u_{1} v_{1}\right) v_{2} \neq 0,\left(u_{1} v_{2}\right) v_{3} \neq 0,\left(u_{1} v_{1}\right) v_{3} \neq 0$. Mostremos que $\left\{u_{1}, u_{1} v_{1}, u_{1} v_{2}, u_{1} v_{3},\left(u_{1} v_{1}\right) v_{2},\left(u_{1} v_{1}\right) v_{3},\left(u_{1} v_{2}\right) v_{3},\left[\left(u_{1} v_{1}\right) v_{2}\right] v_{3}\right\}$ é livre. Suponhamos que $\alpha_{1} \mathrm{u}_{1}+\alpha_{2} \mathrm{u}_{1} \mathrm{v}_{1}+\alpha_{3} \mathrm{u}_{1} \mathrm{v}_{2}+\alpha_{4} \mathrm{u}_{1} \mathrm{v}_{3}+\alpha_{5}\left(\mathrm{u}_{1} \mathrm{v}_{1}\right) \mathrm{v}_{2}+$ $+\alpha_{6}\left(\mathrm{u}_{1} \mathrm{v}_{1}\right) \mathrm{v}_{3}+\alpha_{7}\left(\mathrm{u}_{1} \mathrm{v}_{2}\right) \mathrm{v}_{3}+\alpha_{8}\left[\left(\mathrm{u}_{1} \mathrm{v}_{1}\right) \mathrm{v}_{2}\right] \mathrm{v}_{3}=0$. Dessa forma, ao multiplicarmos por $\mathrm{v}_{1}$, de (1.15) e (4.3) obtemos $\alpha_{1} \mathrm{u}_{1} \mathrm{v}_{1}+\alpha_{3}\left(\mathrm{u}_{1} \mathrm{v}_{2}\right) \mathrm{v}_{1}+$ $+\alpha_{4}\left(\mathrm{u}_{1} \mathrm{v}_{3}\right) \mathrm{v}_{1}+\alpha_{7}\left[\left(\mathrm{u}_{1} \mathrm{v}_{2}\right) \mathrm{v}_{3}\right] \mathrm{v}_{1}=0$ e portanto, multiplicando agora por $\mathrm{v}_{2}$ e novamente de (1.15) e (4.3), $\alpha_{1}\left(\mathrm{u}_{1} \mathrm{v}_{1}\right) \mathrm{v}_{2}+\alpha_{4}\left[\left(\mathrm{u}_{1} \mathrm{v}_{3}\right) \mathrm{v}_{1}\right] \mathrm{v}_{2}=0$. Assim, multiplicando por $\mathrm{v}_{3}$ e como nos casos anteriores, $\alpha_{1}\left[\left(\mathrm{u}_{1} \mathrm{v}_{1}\right) \mathrm{v}_{2}\right] \mathrm{v}_{3}=0$ : ou seja, $\alpha_{1}=\alpha_{4}=0$ e isto mostra que $\left\{\left(u_{1} v_{1}\right) v_{2},\left[\left(u_{1} v_{1}\right) v_{2}\right] v_{3}\right\}$ é livre. Segue 
então que $\alpha_{3}\left(\mathrm{u}_{1} \mathrm{v}_{2}\right) \mathrm{v}_{1}+\alpha_{7}\left[\left(\mathrm{u}_{1} \mathrm{v}_{2}\right) \mathrm{v}_{3}\right] \mathrm{v}_{1}=0$ e, pela observação acima, como $\left(\right.$ por (4.3)) $\left(\mathrm{u}_{1} \mathrm{v}_{2}\right) \mathrm{v}_{1}=-\left(\mathrm{u}_{1} \mathrm{v}_{1}\right) \mathrm{v}_{2}$, obtemos $\alpha_{3}=\alpha_{\bar{\tau}}=0$. Logo, $\alpha_{2} \mathrm{u}_{1} \mathrm{v}_{1}+$ $+\alpha_{5}\left(\mathrm{u}_{1} \mathrm{v}_{1}\right) \mathrm{v}_{2}+\alpha_{6}\left(\mathrm{u}_{1} \mathrm{v}_{1}\right) \mathrm{v}_{3}+\alpha_{8}\left[\left(\mathrm{u}_{1} \mathrm{v}_{1}\right) \mathrm{v}_{2}\right] \mathrm{v}_{3}=0$ e assim, multiplicando finalmente por $\mathrm{v}_{2}, \alpha_{2}\left(\mathrm{u}_{1} \mathrm{v}_{1}\right) \mathrm{v}_{2}+\alpha_{6}\left[\left(\mathrm{u}_{1} \mathrm{v}_{1}\right) \mathrm{v}_{3}\right] \mathrm{v}_{2}=0$; ou seja, $\alpha_{2}=\alpha_{6}=$ $=0$ e $\alpha_{5}\left(\mathrm{u}_{1} \mathrm{v}_{1}\right) \mathrm{v}_{2}+\alpha_{8}\left[\left(\mathrm{u}_{1} \mathrm{v}_{1}\right) \mathrm{v}_{2}\right] \mathrm{v}_{3}=0$, o que implica em $\alpha_{5}=\alpha_{8}=0$ e portanto $\operatorname{dim} \mathrm{U} \geq 8$. Por outro lado, $\operatorname{dim} \mathrm{V} \geq 3$, uma vez que $\left\{\mathrm{v}_{1}, \mathrm{v}_{2}\right.$, $\left.\mathrm{v}_{3}\right\}$ é livre, pois sua imagem pelo operador $\mathrm{L}_{\mathrm{u}_{1}}$ é livre, conforme provamos acima.

Exemplo 14: A álgebra A com base $\left\{e, u_{1}, u_{2}, u_{3}, u_{4}, u_{5}, u_{6}, u_{7}, u_{8}, v_{1}\right.$, $\left.\mathrm{v}_{2}, \mathrm{v}_{3}\right\}$ e tábua de multiplicação

$$
\left\{\begin{array}{l}
\mathrm{e}^{2}=\mathrm{e} ; 2 \mathrm{eu}_{\mathrm{i}}=\mathrm{u}_{\mathrm{i}}(\mathrm{i}=1, \ldots, 8) ; \quad \mathrm{v}_{\mathrm{i}}=0(\mathrm{i}=1,2,3) \\
\mathrm{u}_{1} \mathrm{v}_{1}=\mathrm{u}_{2} ; \quad \mathrm{u}_{1} \mathrm{v}_{2}=\mathrm{u}_{3} ; \quad \mathrm{u}_{1} \mathrm{v}_{3}=\mathrm{u}_{4} \\
\mathrm{u}_{2} \mathrm{v}_{2}=\mathrm{u}_{5} ; \quad \mathrm{u}_{2} \mathrm{v}_{3}=\mathrm{u}_{6} \\
\mathrm{u}_{3} \mathrm{v}_{1}=-\mathrm{u}_{5} ; \quad \mathrm{u}_{3} \mathrm{v}_{3}=\mathrm{u}_{7} \\
\mathrm{u}_{4} \mathrm{v}_{1}=-\mathrm{u}_{6} ; \quad \mathrm{u}_{4} \mathrm{v}_{2}=-\mathrm{u}_{7} \\
\mathrm{u}_{5} \mathrm{v}_{3}=-\mathrm{u}_{6} \mathrm{v}_{2}=\mathrm{u}_{7} \mathrm{v}_{1}=\mathrm{u}_{8} \\
\text { os outros produtos são nulos }
\end{array}\right.
$$

satisfaz $\mathrm{x}^{3}=\omega(\mathrm{x}) \mathrm{x}^{2}, \operatorname{com}[(\mathrm{UV}) \mathrm{V}] \mathrm{V} \neq 0\left(\right.$ pois $\left[\left(\mathrm{u}_{1} \mathrm{v}_{1}\right) \mathrm{v}_{2}\right] \mathrm{v}_{3}=\left(\mathrm{u}_{2} \mathrm{v}_{2}\right) \mathrm{v}_{3}=$ $\left.=\mathrm{u}_{5} \mathrm{v}_{3}=\mathrm{u}_{8}\right)$ e dimensão 12 .

Corolário 4.13.1 Nas condições do Lema 4.13, se $[(U V) V] V$ tem um. gerador aditivo $\left[\left(u_{1} v_{1}\right) v_{2}\right] v_{3}$ tal que $u_{1}^{2} \neq 0$, então $r \geq 8, s \geq 4$ e portanto $\operatorname{dim} A \geq 13$.

Prova: Mostremos que $\operatorname{dim} \mathrm{V} \geq 4$. Para isto basta observar que, neste caso, $\mathrm{u}_{1}^{2}$ não depende linearmente de $\mathrm{v}_{1}, \mathrm{v}_{2}, \mathrm{v}_{3}$. De fato, se $\mathrm{u}_{1}^{2}=\alpha_{1} \mathrm{v}_{1}+$ $+\alpha_{2} \mathrm{v}_{2}+\alpha_{3} \mathrm{v}_{3}$ então, da multiplicação por $\mathrm{u}_{1}$ obtemos $0=\mathrm{u}_{1}^{3}=\alpha_{1} \mathrm{u}_{1} \mathrm{v}_{1}+$ $+\alpha_{2} \mathrm{u}_{1} \mathrm{v}_{2}+\alpha_{3} \mathrm{u}_{1} \mathrm{v}_{3}$ e daí $\alpha_{1}=\alpha_{2}=\alpha_{3}=0$ (pelo que provamos acima), 
e isto acarreta $u_{1}^{2}=0$, o que é um absurdo.

Exemplo 15: A álgebra A com base $\left\{e, u_{1}, u_{2}, u_{3}, u_{4}, u_{5}, u_{6}, u_{7}, u_{8}, v_{1}\right.$, $\left.\mathrm{v}_{2}, \mathrm{v}_{3}, \mathrm{v}_{4}\right\}$ e tábua de multiplicação

$$
\left\{\begin{array}{l}
\mathrm{e}^{2}=\mathrm{e} ; 2 \mathrm{eu}_{\mathrm{i}}=\mathrm{u}_{\mathrm{i}}(\mathrm{i}=1, \ldots, 8) ; \quad \mathrm{v}_{\mathrm{i}}=0(\mathrm{i}=1, \ldots, 4) \\
\mathrm{u}_{1}^{2}=\mathrm{v}_{4} \\
\mathrm{u}_{1} \mathrm{v}_{1}=\mathrm{u}_{2} ; \quad \mathrm{u}_{1} \mathrm{v}_{2}=\mathrm{u}_{3} ; \quad \mathrm{u}_{1} \mathrm{v}_{3}=\mathrm{u}_{4} \\
\mathrm{u}_{2} \mathrm{v}_{2}=\mathrm{u}_{5} ; \quad \mathrm{u}_{2} \mathrm{v}_{3}=\mathrm{u}_{6} \\
\mathrm{u}_{3} \mathrm{v}_{1}=-\mathrm{u}_{5} ; \quad \mathrm{u}_{3} \mathrm{v}_{3}=\mathrm{u}_{7} \\
\mathrm{u}_{4} \mathrm{v}_{1}=-\mathrm{u}_{6} ; \quad \mathrm{u}_{4} \mathrm{v}_{2}=-\mathrm{u}_{7} \\
\mathrm{u}_{5} \mathrm{v}_{3}=-\mathrm{u}_{6} \mathrm{v}_{2}=\mathrm{u}_{7} \mathrm{v}_{1}=\mathrm{u}_{8} \\
\text { os outros produtos são nulos }
\end{array}\right.
$$

satisfaz $\mathrm{x}^{3}=\omega(\mathrm{x}) \mathrm{x}^{2}, \quad$ com $[(\mathrm{UV}) \mathrm{V}] \mathrm{V} \neq 0 \quad\left(\right.$ pois $\left[\left(\mathrm{u}_{1} \mathrm{v}_{1}\right) \mathrm{v}_{2}\right] \mathrm{v}_{3}=$ $\left.=\left(\mathrm{u}_{2} \mathrm{v}_{2}\right) \mathrm{v}_{3}=\mathrm{u}_{5} \mathrm{v}_{3}=\mathrm{u}_{8}\right), \mathrm{u}_{1}^{2} \neq 0$ e dimensão 13 .

Os lemas acima, bem como os exemplos apresentados, resolvem o problema de estimar a mínima dimensão de uma t-álgebra de posto 3 , conhecendo um $\mathrm{P}$-monômio não nulo de grau $\leq 4$.

O Lema seguinte é uma generalização do Lema 4.13.

Lema 4.14 Se o P-monômio $U V^{(n)}$ é não nulo, então $r \geq 2^{n}, s \geq n$ e portanto $\operatorname{dim} A \geq 2^{n}+n+1$.

Prova: A prova será feita por indução sobre $n$. Para $n=1,2,3$, o resultado é verdadeiro pelos Lemas 4.5, 4.8 e 4.13, respectivamente. Suponhamos, agora, que se $U V^{(n-1)} \neq 0$ então $\operatorname{dim} U \geq 2^{n-1}$ e $\operatorname{dim} \mathrm{V} \geq \mathrm{n}-1$. Se $\left\{\left[\left(\mathrm{uv}_{1}\right) \mathrm{v}_{2}\right] \ldots \mathrm{v}_{n-1}\right\} \mathrm{v}_{n} \neq 0$, então $\left\{\left[\left(\mathrm{uv}_{1}\right) \mathrm{v}_{2}\right] \ldots\right\} \mathrm{v}_{n-1} \neq 0$ e portanto, pela hipótese de indução, existem em $\mathrm{U}$ pelo menos $2^{n-1}$ elementos linearmente 
independentes. Esses elementos envolvem $\mathrm{u}, \mathrm{v}_{1}, \mathrm{v}_{2}, \ldots, \mathrm{v}_{n-1}$. São eles: $\mathrm{u}, \mathrm{u}_{2}, \mathrm{u}_{3}, \ldots, \mathrm{u}_{2^{\mathrm{n}-1}}$. Afirmo que $\left\{\mathrm{u}, \mathrm{u}_{2}, \mathrm{u}_{3}, \ldots, \mathrm{u}_{2^{\mathrm{n}-1}}, \mathrm{uv}_{n}, \mathrm{u}_{2} \mathrm{v}_{n}, \mathrm{u}_{3} \mathrm{v}_{n}\right.$, $\left.\ldots, \mathrm{u}_{2^{\mathrm{n}-1} \mathrm{v}_{n}}\right\}$ é livre. De fato, se $\alpha \mathrm{u}+\alpha_{2} \mathrm{u}_{2}+\alpha_{3} \mathrm{u}_{3}+\ldots+\alpha_{2^{\mathrm{n}-1} \mathrm{u}_{2^{\mathrm{n}-1}}}+$ $+\beta \mathrm{uv}_{n}+\beta_{2} \mathrm{u}_{2} \mathrm{v}_{n}+\ldots+\beta_{2^{\mathrm{n}-1} \mathrm{u}_{2^{\mathrm{n}-1}} \mathrm{v}_{n}}=0$, então, multiplicando-se por $\mathrm{v}_{n}$, obtemos

$$
\alpha \mathrm{uv}_{n}+\alpha_{2} \mathrm{u}_{2} \mathrm{v}_{n}+\alpha_{3} \mathrm{u}_{3} \mathrm{v}_{n}+\ldots+\alpha_{2^{\mathrm{n}-1} \mathrm{u}_{2^{\mathrm{n}-1}} \mathrm{v}_{n}}=0
$$

Multiplicando-se (i) sucessivamente por $\mathrm{v}_{1}, \mathrm{v}_{2}, \mathrm{v}_{3}, \ldots, \mathrm{v}_{n-1}$, obteremos $\alpha\left\{\left[\left(\mathrm{uv}_{n}\right) \mathrm{v}_{1}\right] \mathrm{v}_{2} \ldots\right\} \mathrm{v}_{n-1}=0$ e portanto $\alpha=0$. Logo $\alpha_{2} \mathrm{u}_{2} \mathrm{v}_{n}+\alpha_{3} \mathrm{u}_{3} \mathrm{v}_{n}+$ $\ldots+\alpha_{2^{n-1}} \mathrm{u}_{2^{\mathrm{n}-1} \mathrm{v}_{n}}=0$ e como $\mathrm{u}_{2} \neq \mathrm{u}_{j}$ para todo $j \neq 2\left(\right.$ pois $\left\{\mathrm{u}_{2}, \mathrm{u}_{j}\right\}$ é livre), para cada $\mathrm{i}=3,4, \ldots 2^{n-1}$ existe $j$ tal que $\left[\left(\mathrm{u}_{i} \mathrm{v}_{n}\right)\right] \ldots \mathrm{v}_{j}=0$ e $\left[\left(\mathrm{u}_{2} \mathrm{v}_{n}\right)\right] \ldots \mathrm{v}_{j} \neq 0$. Daí $\alpha_{2}=0$ e analogamente $\alpha_{3}=\alpha_{4}=\ldots=\alpha_{2^{n-1}}=$ $=0$. Portanto $\beta \mathrm{uv}_{n}+\beta_{2} \mathrm{u}_{2} \mathrm{v}_{n}+\ldots+\beta_{2^{\mathrm{n}-1} \mathrm{u}_{2^{\mathrm{n}-1}} \mathrm{v}_{n}}=0$, isto é, recaimos em (i) e portanto $\beta=\beta_{2}=\ldots=\beta_{2^{n-1}}=0$. Dessa forma, $\left\{u, u_{2}, u_{3}\right.$, $\left.\ldots, \mathrm{u}_{2^{\mathrm{n}-1}}, \mathrm{uv}_{n}, \mathrm{u}_{2} \mathrm{v}_{n}, \mathrm{u}_{3} \mathrm{v}_{n}, \ldots, \mathrm{u}_{2^{\mathrm{n}-1}} \mathrm{v}_{n}\right\}$ é livre e assim $\operatorname{dim} \mathrm{U} \geq 2^{n-1}+$ $+2^{n-1}=2\left(2^{n-1}\right)=2^{n}$. Mostremos agora que $\left\{\mathrm{v}_{1}, \mathrm{v}_{2}, \ldots, \mathrm{v}_{n}\right\}$ é livre. De fato, se $\alpha_{1} \mathrm{v}_{1}+\alpha_{2} \mathrm{v}_{2}+\ldots+\alpha_{n} \mathrm{v}_{n}=0$, então $\alpha_{1} \mathrm{uv}_{1}+\alpha_{2} \mathrm{uv}_{2}+\ldots+$ $+\alpha_{n} \mathrm{uv}_{n}=0$. Multiplicando-se sucessivamente por $\mathrm{v}_{2}, \mathrm{v}_{3}, \ldots, \mathrm{v}_{n}$ segue que $\alpha_{1}\left\{\left[\left(\mathrm{uv}_{1}\right) \mathrm{v}_{2}\right] \ldots\right\} \mathrm{v}_{n}=0$ e portanto $\alpha_{1}=0$. Logo, $\alpha_{2} \mathrm{uv}_{2}+\alpha_{3} \mathrm{uv}_{3}+$ $+\ldots+\alpha_{n} \mathrm{uv}_{n}=0$. Multiplicando-se sucessivamente por $\mathrm{v}_{3}, \mathrm{v}_{4}, \ldots, \mathrm{v}_{n}$ obteremos $\alpha_{2}=0$ e repetindo-se o processo chegaremos em $\alpha_{3}=\alpha_{4}=$ $\ldots=\alpha_{n}=0$. Logo, $\operatorname{dim} \mathrm{V} \geq \mathrm{n}$ e portanto $\operatorname{dim} \mathrm{A} \geq 2^{n}+\mathrm{n}+1$.

Corolário 4.14.1 Nas condiçôes do Lema 4.14, se $U V^{(n)}$ tem um gerador aditivo $\left\{\left[\left(u v_{1}\right) v_{2}\right] \ldots\right\} v_{n} \neq 0$ tal que $u^{2} \neq 0$, então $s \geq n+1$ e portanto $\operatorname{dim} A \geq 2^{n}+n+2$.

Prova: O conjunto $\left\{\mathrm{v}_{1}, \mathrm{v}_{2}, \ldots, \mathrm{v}_{n}, \mathrm{u}^{2}\right\}$ é livre pois se $\alpha_{1} \mathrm{v}_{1}+\alpha_{2} \mathrm{v}_{2}+$ $\ldots+\alpha_{n} \mathrm{v}_{n}+\beta \mathrm{u}^{2}=0$ então $\alpha_{1} \mathrm{uv}_{1}+\alpha_{2} \mathrm{uv}_{2}+\ldots+\alpha_{n} \mathrm{uv}_{n}=0$. Se multiplicarmos essa última igualdade sucessivamente por $\mathrm{v}_{2}, \mathrm{v}_{3}, \ldots, \mathrm{v}_{n}$, obtemos $\alpha_{1}=0$ e $\alpha_{2} \mathrm{uv}_{2}+\alpha_{3} \mathrm{uv}_{3}+\ldots+\alpha_{n} \mathrm{uv}_{n}=0$. Multiplicando-se agora essa última igualdade sucessivamente por $\mathrm{v}_{3}, \ldots, \mathrm{v}_{n}$, obtemos $\alpha_{2}=0$. 
Repetindo o processo, teremos $\alpha_{i}=0$ para todo $\mathrm{i}=1,2, \ldots, \mathrm{n}$ e portanto $\beta \mathrm{u}^{2}=0$. Logo, $\beta=0$ e dim $\mathrm{V} \geq \mathrm{n}+1$. Como já vimos que $\operatorname{dim} \mathrm{U} \geq 2^{n}$, segue que $\operatorname{dim} \mathrm{A} \geq 2^{n}+\mathrm{n}+2$.

Conforme vimos acima, se a t-álgebra A possui um P-monômio não nulo $\mathrm{m}(\mathrm{U}, \mathrm{V})$ então sua dimensão está acima de um certo valor mínimo, isto é, podemos ter certeza de que A está em uma certa região do triângulo dos tipos da página 7. Em geral, concluimos que $\mathrm{A}$ está mais à esquerda do triângulo. Um problema mais difícil é estimar a dimensão de $\mathrm{A}$ a partir de uma informação sobre a dimensão de $\mathrm{m}(\mathrm{U}, \mathrm{V})$. Por exemplo, como fica o Lema 4.13 se sabemos que $\operatorname{dim}[(\mathrm{UV}) \mathrm{V}] \mathrm{V}=2$. 


\section{Capítulo 5}

\section{Sobre a invariância dos P-subespaços}

Neste capítulo, vamos estudar o problema da invariância da dimensão para todos os subespaços $\mathrm{p}(\mathrm{U}, \mathrm{V})$ de grau $\leq 4$. Porém estudamos também o problema da invariância para algumas famílias de P-subespaços de grau arbitrário.

\subsection{Os P-subespaços de grau $\leq 4$}

Seja $\mathrm{A}=\mathrm{Fe} \oplus \mathrm{U} \oplus \mathrm{V}$, uma t-álgebra de posto 3 decomposta segundo um idempotente $e$. Considerando que $\mathrm{V}^{2}=0$, os $\mathrm{P}$-monômios não nulos de $\mathrm{A}$ de graus 2, 3, 4 são em número de 10:

grau 2: $\mathrm{U}^{2}$ e UV

grau 3: $\mathrm{U}^{3}, \mathrm{U}(\mathrm{UV})$ e $(\mathrm{UV}) \mathrm{V}$

grau 4: $\mathrm{U}^{4}, \mathrm{U}[\mathrm{U}(\mathrm{UV})], \mathrm{U}[(\mathrm{UV}) \mathrm{V}]=(\mathrm{UV})^{2}, \mathrm{U}^{3} \mathrm{~V}=\mathrm{U}^{2}(\mathrm{UV})$ e $[(\mathrm{UV}) \mathrm{V}] \mathrm{V}$

A partir deles, mais $\mathrm{U}$ e $\mathrm{V}$, formamos uma lista de $89 \mathrm{P}$-subespaços $\mathrm{p}(\mathrm{U}, \mathrm{V})$ de grau $\leq 4$, que aparecem listados no Apêndice. Para cada um deles, vamos tentar verificar se é invariante ou, pelo menos, se tem dimensão invariante. A demonstração é feita caso a caso, por isso temos um grande número de lemas.

Observamos que, se $\mathrm{e}_{0}=\mathrm{e}+\mathrm{u}_{0}+\lambda \mathrm{u}_{0}^{2}$ é um outro idempotente de $\mathrm{A}$ 
e a correspondente decomposição de Peirce é $\mathrm{A}=\mathrm{Fe}_{0} \oplus \mathrm{U}_{0} \oplus \mathrm{V}_{0}$. então temos dois $\mathrm{P}$-subespaços $\mathrm{p}(\mathrm{U}, \mathrm{V})$ e $\mathrm{p}\left(\mathrm{U}_{0}, \mathrm{~V}_{0}\right)$, sobre os quais queremos trabalhar. Em geral, existe aqui um "princípio de simetria" no sentido de que se desejamos, por exemplo, provar que $\mathrm{p}(\mathrm{U}, \mathrm{V})=\mathrm{p}\left(\mathrm{U}_{0}, \mathrm{~V}_{0}\right)$, basta mostrar que $\mathrm{p}\left(\mathrm{U}_{0}, \mathrm{~V}_{0}\right) \subseteq \mathrm{p}(\mathrm{U}, \mathrm{V})$, a outra inclusão sendo obtida "por simetria".

\subsection{Proposições auxiliares: geradores de $\mathrm{m}(\mathrm{U}, \mathrm{V})$}

Para começar a estudar o problema de invariância, vamos calcular um conjunto conveniente de geradores aditivos para os $10 \mathrm{P}$-monômios $\mathrm{m}\left(\mathrm{U}_{\mathbf{0}}, \mathrm{V}_{\mathbf{0}}\right)$ de graus 2,3 ou 4 listados no início da seção. Isto será feito nas Proposições 1 a 10 que seguem. Para isso, consideremos a decomposição de Peirce de $\mathrm{A}$ relativamente ao idempotente $e, \mathrm{~A}=\mathrm{Fe} \oplus$ $\oplus \mathrm{U} \oplus \mathrm{V}$ e $\mathrm{e}_{0}=\mathrm{e}+\mathrm{u}_{0}+\lambda \mathrm{u}_{0}^{2}$ um outro idempotente fixado de A, sendo $\lambda=(1-2 \gamma)^{-1}$. Como já vimos em (1.20) e (1.21),

$$
\begin{aligned}
& \mathrm{U}_{0}=\left\{\mathrm{u}+2 \lambda \mathrm{u}_{0} \mathrm{u}: \mathrm{u} \in \mathrm{U}\right\} \\
& \mathrm{V}_{0}=\left\{\mathrm{v}-2 \lambda \mathrm{u}_{0} \mathrm{v}: \mathrm{v} \in \mathrm{V}\right\}
\end{aligned}
$$

e se $u, u_{1} \in U$ e $v \in V$ valem as igualdades $u(u v)=u^{2}(u v)=u\left(u^{2} u_{1}\right)=$ $=0$. Usamos o símbolo $\oplus$ para indicar uma soma de um elemento de $\mathrm{U}$ e um elemento de V. Cremos que isto facilita a leitura do texto.

Proposição $1 O$ subespaço vetorial $U_{0} V_{0}$ é gerado aditivamente pelos elementos da forma $\left[u v+2 \lambda^{2} u_{0}^{2}(u v)\right] \oplus 2 \lambda u_{0}(u v)$, com $u \in U$ e $v \in V$.

Prova: De fato, um elemento gerador aditivo $y$ de $\mathrm{U}_{0} \mathrm{~V}_{0}$ é da forma

$$
\begin{aligned}
\mathrm{y} & =\left(\mathrm{u}+2 \lambda \mathrm{u}_{0} \mathrm{u}\right)\left(\mathrm{v}-2 \lambda \mathrm{u}_{0} \mathrm{v}\right)= \\
& =\mathrm{uv}-2 \lambda \mathrm{u}\left(\mathrm{u}_{0} \mathrm{v}\right)+2 \lambda\left(\mathrm{u}_{0} \mathrm{u}\right) \mathrm{v}-4 \lambda^{2}\left(\mathrm{u}_{0} \mathrm{u}\right)\left(\mathrm{u}_{0} \mathrm{v}\right)= \\
& =\mathrm{uv}-2 \lambda \mathrm{u}\left(\mathrm{u}_{0} \mathrm{v}\right)-4 \lambda^{2}\left(\mathrm{u}_{0} \mathrm{u}\right)\left(\mathrm{u}_{0} \mathrm{v}\right)=\mathrm{uv}+2 \lambda \mathrm{u}_{0}(\mathrm{uv})+2 \lambda^{2} \mathrm{u}_{0}^{2}(\mathrm{uv}),
\end{aligned}
$$

sendo esta última igualdade consequência de (4.4) e (4.2). Daí segue o resultado. 
Proposição $2 O$ subespaço vetorial $U_{0}\left(U_{0} V_{0}\right)$ é gerado pelos elementos do tipo $\left\{-2 \lambda u_{0}\left[u_{1}(u v)\right]+4 \lambda^{3} u_{0}\left[\left(u_{1} u_{0}^{2}\right)(u v)\right]\right\} \oplus\left\{u_{1}(u v)-2 \lambda^{2}\left(u_{1} u_{0}^{2}\right)(u v)\right\}$, com $u, u_{1} \in U$ e $v \in V$.

Prova: De acordo com a Proposição $1, \mathrm{U}_{0} \mathrm{~V}_{0}$ é gerado pelos elementos da forma [uv $\left.+2 \lambda^{2} \mathrm{u}_{0}^{2}(\mathrm{uv})\right]+2 \lambda \mathrm{u}_{0}(\mathrm{uv})$, e portanto um gerador aditivo $y$ de $\mathrm{U}_{0}\left(\mathrm{U}_{0} \mathrm{~V}_{0}\right)$ é da forma

$$
\begin{aligned}
\mathrm{y}= & \left(\mathrm{u}_{1}+2 \lambda \mathrm{u}_{0} \mathrm{u}_{1}\right)\left[\mathrm{uv}+2 \lambda^{2} \mathrm{u}_{0}^{2}(\mathrm{uv})+2 \lambda \mathrm{u}_{0}(\mathrm{uv})\right]= \\
= & \mathrm{u}_{1}(\mathrm{uv})+2 \lambda^{2} \mathrm{u}_{1}\left[\mathrm{u}_{0}^{2}(\mathrm{uv})\right]+2 \lambda \mathrm{u}_{1}\left[\mathrm{u}_{0}(\mathrm{uv})\right]+2 \lambda\left(\mathrm{u}_{0} \mathrm{u}_{1}\right)(\mathrm{uv})+ \\
& +4 \lambda^{3}\left(\mathrm{u}_{0} \mathrm{u}_{1}\right)\left[\mathrm{u}_{0}^{2}(\mathrm{uv})\right],
\end{aligned}
$$

pois $\left(\mathrm{u}_{0} \mathrm{u}_{1}\right)\left[\mathrm{u}_{0}(\mathrm{uv})\right] \in \mathrm{U}^{2}[\mathrm{U}(\mathrm{UV})] \subseteq \mathrm{V} \mathrm{U} \mathrm{U}^{2} \subseteq \mathrm{V}^{2}=0$. Vamos agora analisar o termo $2 \lambda \mathrm{u}_{1}\left[\mathrm{u}_{0}(\mathrm{uv})\right]+2 \lambda\left(\mathrm{u}_{0} \mathrm{u}_{1}\right)(\mathrm{uv})$. Usando-se a identidade (1.13) para os elementos $\mathrm{u}_{1}, \mathrm{u}_{0}, \mathrm{uv}$, obtemos $\mathrm{u}_{1}\left[\mathrm{u}_{0}(\mathrm{uv})\right]+\mathrm{u}_{0}\left[\mathrm{u}_{1}(\mathrm{uv})\right]+$ $+\left(\mathrm{u}_{0} \mathrm{u}_{1}\right)(\mathrm{uv})=0$ e portanto

$$
2 \lambda \mathrm{u}_{1}\left[\mathrm{u}_{0}(\mathrm{uv})\right]+2 \lambda\left(\mathrm{u}_{0} \mathrm{u}_{1}\right)(\mathrm{uv})=-2 \lambda \mathrm{u}_{0}\left[\mathrm{u}_{1}(\mathrm{uv})\right]
$$

Além disso, de (4.4) segue que

$$
\mathrm{u}_{1}\left[\mathrm{u}_{0}^{2}(\mathrm{uv})\right]=-\left(\mathrm{u}_{1} \mathrm{u}_{0}^{2}\right)(\mathrm{uv})
$$

Finalmente, da aplicação sucessiva de (4.3), (4.1), (4.4) e novamente (4.1) segue que $\left(\mathrm{u}_{0} \mathrm{u}_{1}\right)\left[\mathrm{u}_{0}^{2}(\mathrm{uv})\right]=-\mathrm{u}_{0}^{2}\left[\left(\mathrm{u}_{0} \mathrm{u}_{1}\right)(\mathrm{uv})\right]=2 \mathrm{u}_{0}\left\{\mathrm{u}_{0}\left[\left(\mathrm{u}_{0} \mathrm{u}_{1}\right)(\mathrm{uv})\right]\right\}=$ $=-2 \mathrm{u}_{0}\left\{(\mathrm{uv})\left[\mathrm{u}_{0}\left(\mathrm{u}_{0} \mathrm{u}_{1}\right)\right]\right\}=\mathrm{u}_{0}\left[(\mathrm{uv})\left(\mathrm{u}_{0}^{2} \mathrm{u}_{1}\right)\right]$, ou seja,

$$
\left(\mathrm{u}_{0} \mathrm{u}_{1}\right)\left[\mathrm{u}_{0}^{2}(\mathrm{uv})\right]=\mathrm{u}_{0}\left[\left(\mathrm{u}_{1} \mathrm{u}_{0}^{2}\right)(\mathrm{uv})\right]
$$

Desse modo, de (i), (ii), (iii) segue o resultado.

Proposição $3 O$ subespaço vetorial $\left(U_{0} V_{0}\right) V_{0}$ é gerado aditivamente pelos elementos da forma $(u v) v_{1} \oplus 2 \lambda u_{0}\left[(u v) v_{1}\right]$, com. $u \in U$ e v, $v_{1} \in V$.

Prova: De acordo com a Proposição $1, \mathrm{U}_{0} \mathrm{~V}_{0}$ é gerado pelos elementos da forma $\left[u v+2 \lambda^{2} u_{0}^{2}(u v)\right]+2 \lambda u_{0}(u v)$, e portanto um gerador aditivo y 
de $\left(\mathrm{U}_{0} \mathrm{~V}_{0}\right) \mathrm{V}_{0}$ é da forma

$$
\begin{aligned}
\mathrm{y}= & {\left[\mathrm{uv}+2 \lambda^{2} \mathrm{u}_{0}^{2}(\mathrm{uv})+2 \lambda \mathrm{u}_{0}(\mathrm{uv})\right]\left(\mathrm{v}_{1}-2 \lambda \mathrm{u}_{0} \mathrm{v}_{1}\right)=} \\
= & (\mathrm{uv}) \mathrm{v}_{1}-2 \lambda(\mathrm{uv})\left(\mathrm{u}_{0} \mathrm{v}_{1}\right)+2 \lambda^{2}\left[\mathrm{u}_{0}^{2}(\mathrm{uv})\right] \mathrm{v}_{1}-4 \lambda^{3}\left[\mathrm{u}_{0}^{2}(\mathrm{uv})\right]\left(\mathrm{u}_{0} \mathrm{v}_{1}\right)+ \\
& -4 \lambda^{2}\left[\mathrm{u}_{0}(\mathrm{uv})\right]\left(\mathrm{u}_{0} \mathrm{v}_{1}\right),
\end{aligned}
$$

pois $\left[\mathrm{u}_{0}(\mathrm{uv})\right] \mathrm{v}_{1} \in[\mathrm{U}(\mathrm{UV})] \mathrm{V} \subseteq \mathrm{V}^{2}=0$. Além disso, de (4.1) segue que $\left[\mathrm{u}_{0}^{2}(\mathrm{uv})\right]\left(\mathrm{u}_{0} \mathrm{v}_{1}\right)=-2\left\{\mathrm{u}_{0}\left[\mathrm{u}_{0}(\mathrm{uv})\right]\right\}\left(\mathrm{u}_{0} \mathrm{v}_{1}\right)=0$, sendo esta última igualdade consequência da identidade (4.9). Por outro lado, $\left[\mathrm{u}_{0}^{2}(\mathrm{uv})\right] \mathrm{v}_{1}=$ $=-2\left\{\mathrm{u}_{0}\left[\mathrm{u}_{0}(\mathrm{uv})\right]\right\} \mathrm{v}_{1}=2\left(\mathrm{u}_{0} \mathrm{v}_{1}\right)\left[\mathrm{u}_{0}(\mathrm{uv})\right]$, por (4.1) e (4.3), respectivamente. Portanto, $2 \lambda^{2}\left[\mathrm{u}_{0}^{2}(\mathrm{uv})\right] \mathrm{v}_{1}-4 \lambda^{2}\left[\mathrm{u}_{0}(\mathrm{uv})\right]\left(\mathrm{u}_{0} \mathrm{v}_{1}\right)=0$. Finalmente, de (4.5) segue que $(u v)\left(u_{0} v_{1}\right)=-u_{0}\left[(u v) v_{1}\right]$ e portanto o gerador $y$ se reduz a $(\mathrm{uv}) \mathrm{v}_{1} \oplus 2 \lambda \mathrm{u}_{0}\left[(\mathrm{uv}) \mathrm{v}_{1}\right]$.

Proposição 4 o subespaço vetorial $U_{0}^{2}$ é gerado pelos elementos da forma $-2 \lambda u_{0}\left(u u_{1}\right) \oplus u u_{1}$, com $u, u_{1} \in U$.

Prova: De acordo com (1.20), um gerador aditivo $y$ de $\mathrm{U}_{0}^{2}$ é da forma

$$
\begin{aligned}
\mathrm{y} & =\left(\mathrm{u}+2 \lambda \mathrm{u}_{0} \mathrm{u}\right)\left(\mathrm{u}_{1}+2 \lambda \mathrm{u}_{0} \mathrm{u}_{1}\right)= \\
& =\mathrm{uu}_{1}+2 \lambda \mathrm{u}\left(\mathrm{u}_{0} \mathrm{u}_{1}\right)+2 \lambda \mathrm{u}_{1}\left(\mathrm{u}_{0} \mathrm{u}\right)+4 \lambda^{2}\left(\mathrm{u}_{0} \mathrm{u}\right)\left(\mathrm{u}_{0} \mathrm{u}_{1}\right)= \\
& =\mathrm{uu}_{1}+2 \lambda \mathrm{u}\left(\mathrm{u}_{0} \mathrm{u}_{1}\right)+2 \lambda \mathrm{u}_{1}\left(\mathrm{u}_{0} \mathrm{u}\right),
\end{aligned}
$$

pois $\left(\mathrm{u}_{0} \mathrm{u}\right)\left(\mathrm{u}_{0} \mathrm{u}_{1}\right) \in \mathrm{U}^{2} \mathrm{U}^{2} \subseteq \mathrm{V}^{2}=0$. Além disso, como $\mathrm{J}\left(\mathrm{u}, \mathrm{u}_{0}, \mathrm{u}_{1}\right)=$ $=0$, obtemos $\mathrm{u}_{(}\left(\mathrm{u}_{0} \mathrm{u}_{1}\right)+\mathrm{u}_{1}\left(\mathrm{u}_{0} \mathrm{u}\right)=-\mathrm{u}_{0}\left(\mathrm{uu}_{1}\right)$ e portanto segue que $\mathrm{y}=-2 \lambda \mathrm{u}_{0}\left(\mathrm{uu}_{1}\right) \oplus \mathrm{uu}_{1}$.

Proposição 50 subespaço vetorial $U_{0}^{3}$ é gerado aditivamente pelos elementos da forma $\left\{u_{2}\left(u u_{1}\right)+2 \lambda^{2} u_{0}^{2}\left[u_{2}\left(u u_{1}\right)\right]\right\} \oplus\left\{2 \lambda u_{0}\left[u_{2}\left(u u_{1}\right)\right]\right\}$, com $u, u_{1}, u_{2} \in U$.

Prova: Por (1.20) e pela Proposição 4 acima, um gerador aditivo $y$ de $\mathrm{U}_{0}^{3}$ é da forma

$$
\begin{aligned}
\mathrm{y} & =\left[\mathrm{uu}_{1}-2 \lambda \mathrm{u}_{0}\left(\mathrm{uu}_{1}\right)\right]\left(\mathrm{u}_{2}+2 \lambda \mathrm{u}_{0} \mathrm{u}_{2}\right)= \\
& =\mathrm{u}_{2}\left(\mathrm{uu}_{1}\right)-2 \lambda \mathrm{u}_{2}\left[\mathrm{u}_{0}\left(\mathrm{uu}_{1}\right)\right]-4 \lambda^{2}\left(\mathrm{u}_{0} \mathrm{u}_{2}\right)\left[\mathrm{u}_{0}\left(\mathrm{uu}_{1}\right)\right] .
\end{aligned}
$$

pois $\left(\mathrm{uu}_{1}\right)\left(\mathrm{u}_{0} \mathrm{u}_{2}\right) \in \mathrm{U}^{2} \mathrm{U}^{2} \subseteq \mathrm{V}^{2}=0$. Além disso, da identidade (4.4) obte- 
$\operatorname{mos}-\mathrm{u}_{2}\left[\mathrm{u}_{0}\left(\mathrm{uu}_{1}\right)\right]=\mathrm{u}_{0}\left[\mathrm{u}_{2}\left(\mathrm{uu}_{1}\right)\right]$. Finalmente, da aplicação sucessiva das identidades $(4.3),(4.1)$ e novamente de $(4.3)$, vem que $-2\left(\mathrm{u}_{0} \mathrm{u}_{2}\right)\left[\mathrm{u}_{0}\left(\mathrm{uu}_{1}\right)\right]=$ $=2\left(\mathrm{uu}_{1}\right)\left[\mathrm{u}_{0}\left(\mathrm{u}_{0} \mathrm{u}_{2}\right)\right]=-\left(\mathrm{uu}_{1}\right)\left(\mathrm{u}_{0}^{2} \mathrm{u}_{2}\right)=\mathrm{u}_{0}^{2}\left[\mathrm{u}_{2}\left(\mathrm{uu}_{1}\right)\right]$ e dessa forma segue o resultado.

Proposição 6 O subespaço vetorial $U_{0}^{4}$ é gerado pelos elementos da forma $4 \lambda^{3}\left(u_{0} u_{3}\right)\left\{u_{0}^{2}\left[u_{2}\left(u u_{1}\right)\right]\right\}-2 \lambda u_{0}\left\{u_{3}\left[u_{2}\left(u u_{1}\right)\right]\right\} \oplus u_{3}\left[u_{2}\left(u u_{1}\right)\right]+$ $+2 \lambda^{2} u_{3}\left\{u_{0}^{2}\left[u_{2}\left(u u_{1}\right)\right]\right\}$, com $u, u_{1}, u_{2}, u_{3} \in U$.

Prova: De (1.20) e pela Proposição 5 acima, um elemento $y$ gerador de $\mathrm{U}_{0}{ }^{4}$ é da forma

$$
\begin{aligned}
\mathrm{y}= & \left(\mathrm{u}_{3}+2 \lambda \mathrm{u}_{0} \mathrm{u}_{3}\right)\left\{\mathrm{u}_{2}\left(\mathrm{uu}_{1}\right)+2 \lambda^{2} \mathrm{u}_{0}^{2}\left[\mathrm{u}_{2}\left(\mathrm{uu}_{1}\right)\right]+2 \lambda \mathrm{u}_{0}\left[\mathrm{u}_{2}\left(\mathrm{uu}_{1}\right)\right]\right\}= \\
= & \mathrm{u}_{3}\left[\mathrm{u}_{2}\left(\mathrm{uu}_{1}\right)\right]+2 \lambda^{2} \mathrm{u}_{3}\left\{\mathrm{u}_{0}^{2}\left[\mathrm{u}_{2}\left(\mathrm{uu}_{1}\right)\right]\right\}+2 \lambda \mathrm{u}_{3}\left\{\mathrm{u}_{0}\left[\mathrm{u}_{2}\left(\mathrm{uu}_{1}\right)\right]\right\}+ \\
& +2 \lambda\left(\mathrm{u}_{0} \mathrm{u}_{3}\right)\left[\mathrm{u}_{2}\left(\mathrm{uu}_{1}\right)\right]+4 \lambda^{3}\left(\mathrm{u}_{0} \mathrm{u}_{3}\right)\left\{\mathrm{u}_{0}^{2}\left[\mathrm{u}_{2}\left(\mathrm{uu}_{1}\right)\right]\right\},
\end{aligned}
$$

pois $\left(\mathrm{u}_{0} \mathrm{u}_{3}\right)\left\{\mathrm{u}_{0}\left[\mathrm{u}_{2}\left(\mathrm{uu}_{1}\right)\right]\right\} \in \mathrm{U}^{2} \mathrm{U}^{4} \subseteq \mathrm{V}^{2}=0$. Usando-se a identidade de Jacobi para os elementos $\mathrm{u}_{3}, \mathrm{u}_{0}, \mathrm{u}_{2}\left(\mathrm{uu}_{1}\right)$ obtemos $\mathrm{u}_{3}\left\{\mathrm{u}_{0}\left[\mathrm{u}_{2}\left(\mathrm{uu}_{1}\right)\right]\right\}+$ $+\mathrm{u}_{0}\left\{\mathrm{u}_{3}\left[\mathrm{u}_{2}\left(\mathrm{uu}_{1}\right)\right]\right\}+\left(\mathrm{u}_{0} \mathrm{u}_{3}\right)\left[\mathrm{u}_{2}\left(\mathrm{uu}_{1}\right)\right]=0$ e portanto $2 \lambda \mathrm{u}_{3}\left\{\mathrm{u}_{0}\left[\mathrm{u}_{2}\left(\mathrm{uu}_{1}\right)\right]\right\}+$ $+2 \lambda\left(\mathrm{u}_{0} \mathrm{u}_{3}\right)\left[\mathrm{u}_{2}\left(\mathrm{uu}_{1}\right)\right]=-2 \lambda \mathrm{u}_{0}\left\{\mathrm{u}_{3}\left[\mathrm{u}_{2}\left(\mathrm{uu}_{1}\right)\right]\right\}$. Assim segue o resultado.

Proposição $7 \quad O$ subespaço vetorial $U_{0}^{3} V_{0}$ é gerado pelos elementos da forma $\left[u_{2}\left(u u_{1}\right)\right] v \oplus 2 \lambda u_{0}\left\{\left[u_{2}\left(u u_{1}\right)\right] v\right\}, \operatorname{com} u, u_{1}, u_{2} \in U$ e $v \in V$.

Prova: Pela Proposição 5 e por (1.21), um elemento $y$ gerador de $\mathrm{U}_{0}^{3} \mathrm{~V}_{0}$ é da forma

$$
\begin{aligned}
\mathrm{y}= & \left\{\mathrm{u}_{2}\left(\mathrm{uu}_{1}\right)+2 \lambda^{2} \mathrm{u}_{0}^{2}\left[\mathrm{u}_{2}\left(\mathrm{uu}_{1}\right)\right]+2 \lambda \mathrm{u}_{0}\left[\mathrm{u}_{2}\left(\mathrm{uu}_{1}\right)\right]\right\}\left(\mathrm{v}-2 \lambda \mathrm{u}_{0} \mathrm{v}\right)= \\
= & {\left[\mathrm{u}_{2}\left(\mathrm{uu}_{1}\right)\right] \mathrm{v}+2 \lambda^{2}\left\{\mathrm{u}_{0}^{2}\left[\mathrm{u}_{2}\left(\mathrm{uu}_{1}\right)\right]\right\} \mathrm{v}-2 \lambda\left(\mathrm{u}_{0} \mathrm{v}\right)\left[\mathrm{u}_{2}\left(\mathrm{uu}_{1}\right)\right]+} \\
& -4 \lambda^{3}\left(\mathrm{u}_{0} \mathrm{v}\right)\left\{\mathrm{u}_{0}^{2}\left[\mathrm{u}_{2}\left(\mathrm{uu}_{1}\right)\right]\right\}-4 \lambda^{2}\left(\mathrm{u}_{0} \mathrm{v}\right)\left\{\mathrm{u}_{0}\left[\mathrm{u}_{2}\left(\mathrm{uu}_{1}\right)\right]\right\},
\end{aligned}
$$

pois $\left\{\mathrm{u}_{0}\left[\mathrm{u}_{2}\left(\mathrm{uu}_{1}\right)\right]\right\} \mathrm{v} \subseteq \mathrm{U}^{4} \mathrm{~V} \subseteq \mathrm{V}^{2}=0$. Além disso, como $\mathrm{u}_{2}\left(\mathrm{uu}_{1}\right), \mathrm{u}_{0} \mathrm{v} \in$ $\in U$, de $(4.4)$ e $(1.16)$, respectivamente, segue que $\left(\mathrm{u}_{0} \mathrm{v}\right)\left\{\mathrm{u}_{0}^{2}\left[\mathrm{u}_{2}\left(\mathrm{uu}_{1}\right)\right]\right\}=$ $=-\left[\mathrm{u}_{2}\left(\mathrm{uu}_{1}\right)\right]\left[\mathrm{u}_{0}^{2}\left(\mathrm{u}_{0} \mathrm{v}\right)\right]=0$. Finalmente, da aplicação sucessiva das identidades (4.1) e (4.3) temos que $\left\{\mathrm{u}_{0}^{2}\left[\mathrm{u}_{2}\left(\mathrm{uu}_{1}\right)\right]\right\} \mathrm{v}=-2\left\{\mathrm{u}_{0}\left\{\mathrm{u}_{0}\left[\mathrm{u}_{2}\left(\mathrm{uu}_{1}\right)\right]\right\}\right\} \mathrm{v}=$ $=2\left(\mathrm{u}_{0} \mathrm{v}\right)\left\{\mathrm{u}_{0}\left[\mathrm{u}_{2}\left(\mathrm{uu}_{1}\right)\right]\right\}$, pois $\mathrm{u}_{0}\left[\mathrm{u}_{2}\left(\mathrm{uu}_{1}\right)\right], \mathrm{v} \in \mathrm{V}$. Logo, $2 \lambda^{2}\left\{\mathrm{u}_{0}^{2}\left[\mathrm{u}_{2}\left(\mathrm{uu}_{1}\right)\right]\right\} \mathrm{v}=$ 
$=4 \lambda^{2}\left(\mathrm{u}_{0} \mathrm{v}\right)\left\{\mathrm{u}_{0}\left[\mathrm{u}_{2}\left(\mathrm{uu}_{1}\right)\right]\right\}$ e daí segue o resultado.

Proposição 8 O subespaço vetorial $U_{0}\left[U_{0}\left(U_{0} V_{0}\right)\right]$ é gerado pelos elementos do tipo $u_{2}\left[u_{1}(u v)\right]-4 \lambda^{2} u_{2}\left\{u_{0}\left[\left(u_{0} u_{1}\right)(u v)\right]\right\}+2 \lambda^{2} u_{0}^{2}\left\{u_{2}\left[u_{1}(u v)\right]\right\}+$ $+8 \lambda^{4}\left(u_{0} u_{2}\right)\left\{u_{0}\left[\left(u_{1} u_{0}^{2}\right)(u v)\right]\right\} \oplus 2 \lambda u_{0}\left\{u_{2}\left[u_{1}(u v)\right]\right\}-4 \lambda^{3} u_{0}\left\{u_{2}\left[\left(u_{1} u_{0}^{2}\right)(u v)\right]\right\}$, com $u_{1}, u_{2}, u \in U$ e $v \in V$.

Prova: Pela Proposição 2 e por (1.20), um elemento $y$ gerador de $\mathrm{U}_{0}\left[\mathrm{U}_{0}\left(\mathrm{U}_{0} \mathrm{~V}_{0}\right)\right]$ é da forma

$$
\begin{aligned}
\mathrm{y}= & \left(\mathrm{u}_{2}+2 \lambda \mathrm{u}_{0} \mathrm{u}_{2}\right)\left\{-2 \lambda \mathrm{u}_{0}\left[\mathrm{u}_{1}(\mathrm{uv})\right]+4 \lambda^{3} \mathrm{u}_{0}\left[\left(\mathrm{u}_{1} \mathrm{u}_{0}^{2}\right)(\mathrm{uv})\right]+\mathrm{u}_{1}(\mathrm{uv})+\right. \\
& \left.\left.-2 \lambda^{2}\left(\mathrm{u}_{1} \mathrm{u}_{0}^{2}\right)(\mathrm{uv})\right]\right\}= \\
= & -2 \lambda \mathrm{u}_{2}\left\{\mathrm{u}_{0}\left[\mathrm{u}_{1}(\mathrm{uv})\right]\right\}+4 \lambda^{3} \mathrm{u}_{2}\left\{\mathrm{u}_{0}\left[\left(\mathrm{u}_{1} \mathrm{u}_{0}^{2}\right)(\mathrm{uv})\right]\right\}+\mathrm{u}_{2}\left[\mathrm{u}_{1}(\mathrm{uv})\right]+ \\
& -2 \lambda^{2} \mathrm{u}_{2}\left[\left(\mathrm{u}_{1} \mathrm{u}_{0}^{2}\right)(\mathrm{uv})\right]-4 \lambda^{2}\left(\mathrm{u}_{0} \mathrm{u}_{2}\right)\left\{\mathrm{u}_{0}\left[\mathrm{u}_{1}(\mathrm{uv})\right]\right\}+ \\
& +8 \lambda^{4}\left(\mathrm{u}_{0} \mathrm{u}_{2}\right)\left\{\mathrm{u}_{0}\left[\left(\mathrm{u}_{1} \mathrm{u}_{0}^{2}\right)(\mathrm{uv})\right]\right\},
\end{aligned}
$$

pois $\left(\mathrm{u}_{0} \mathrm{u}_{2}\right)\left[\mathrm{u}_{1}(\mathrm{uv})\right]=\left(\mathrm{u}_{0} \mathrm{u}_{2}\right)\left[\left(\mathrm{u}_{1} \mathrm{u}_{0}^{2}\right)(\mathrm{uv})\right]=0$, uma vez que ambos pertencem a $\mathrm{V}^{2}$. Além disso, da identidade (4.4), segue que $-\mathrm{u}_{2}\left\{\mathrm{u}_{0}\left[\mathrm{u}_{1}(\mathrm{uv})\right]\right\}=$ $=\mathrm{u}_{0}\left\{\mathrm{u}_{2}\left[\mathrm{u}_{1}(\mathrm{uv})\right]\right\}$ e $\mathrm{u}_{2}\left\{\mathrm{u}_{0}\left[\left(\mathrm{u}_{1} \mathrm{u}_{0}^{2}\right)(\mathrm{uv})\right]\right\}=-\mathrm{u}_{0}\left\{\mathrm{u}_{2}\left[\left(\mathrm{u}_{1} \mathrm{u}_{0}^{2}\right)(\mathrm{uv})\right]\right\}$ e assim

$$
\begin{aligned}
\mathrm{y}= & \mathrm{u}_{2}\left[\mathrm{u}_{1}(\mathrm{uv})\right]+2 \lambda \mathrm{u}_{0}\left\{\mathrm{u}_{2}\left[\mathrm{u}_{1}(\mathrm{uv})\right]\right\}-4 \lambda^{3} \mathrm{u}_{0}\left\{\mathrm{u}_{2}\left[\left(\mathrm{u}_{1} \mathrm{u}_{0}^{2}\right)(\mathrm{uv})\right]\right\}+ \\
& -2 \lambda^{2} \mathrm{u}_{2}\left[\left(\mathrm{u}_{1} \mathrm{u}_{0}^{2}\right)(\mathrm{uv})\right]-4 \lambda^{2}\left(\mathrm{u}_{0} \mathrm{u}_{2}\right)\left\{\mathrm{u}_{0}\left[\mathrm{u}_{1}(\mathrm{uv})\right]\right\}+ \\
& +8 \lambda^{4}\left(\mathrm{u}_{0} \mathrm{u}_{2}\right)\left\{\mathrm{u}_{0}\left[\left(\mathrm{u}_{1} \mathrm{u}_{0}^{2}\right)(\mathrm{uv})\right]\right\}
\end{aligned}
$$

Analisemos agora o termo $t_{1}=u_{2}\left[\left(u_{1} u_{0}^{2}\right)(u v)\right\}$. De (4.1) e (4.4), respectivamente, segue que $t_{1}=-2 \mathrm{u}_{2}\left\{\left[\mathrm{u}_{0}\left(\mathrm{u}_{0} \mathrm{u}_{1}\right)\right](\mathrm{uv})\right\}=2 \mathrm{u}_{2}\left\{\mathrm{u}_{0}\left[\left(\mathrm{u}_{0} \mathrm{u}_{1}\right)(\mathrm{uv})\right]\right\}$. Finalmente, se $t_{2}=2\left(\mathrm{u}_{0} \mathrm{u}_{2}\right)\left\{\mathrm{u}_{0}\left[\mathrm{u}_{1}(\mathrm{uv})\right]\right\}$, então de (4.3), (4.1) e novamente (4.3), temos $\mathrm{t}_{2}=-2\left[\mathrm{u}_{1}(\mathrm{uv})\right]\left[\mathrm{u}_{0}\left(\mathrm{u}_{0} \mathrm{u}_{2}\right)\right]=\left[\mathrm{u}_{1}(\mathrm{uv})\right]\left(\mathrm{u}_{0}^{2} \mathrm{u}_{2}\right)=-\mathrm{u}_{0}^{2}\left\{\mathrm{u}_{2}\left[\mathrm{u}_{1}(\mathrm{uv})\right]\right\}$. Substituindo-se agora as expressões de $t_{1}$ e $t_{2}$ em (iv), segue o resultado enunciado.

Proposição 9 O subespaço vetorial $U_{0}\left[\left(U_{0} V_{0}\right) V_{0}\right]$ é gerado pelos elementos da forma $-2 \lambda u_{0}\left\{u_{1}\left[(u v) v_{1}\right]\right\} \oplus u_{1}\left[\left(u v^{\prime}\right) v_{1}\right]$, com $u, u_{1} \in U$ e $v$. $v_{1} \in V$. 
Prova: Pela Proposição 3 e por (1.20), um elemento $y$ gerador de $\mathrm{U}_{0}\left[\left(\mathrm{U}_{0} \mathrm{~V}_{0}\right) \mathrm{V}_{0}\right]$ é da forma

$$
\begin{aligned}
\mathrm{y} & =\left(\mathrm{u}_{1}+2 \lambda \mathrm{u}_{0} \mathrm{u}_{1}\right)\left\{(\mathrm{uv}) \mathrm{v}_{1}+2 \lambda \mathrm{u}_{0}\left[(\mathrm{uv}) \mathrm{v}_{1}\right]\right\}= \\
& =\mathrm{u}_{1}\left[(\mathrm{uv}) \mathrm{v}_{1}\right]+2 \lambda \mathrm{u}_{1}\left\{\mathrm{u}_{0}\left[(\mathrm{uv}) \mathrm{v}_{1}\right]\right\}+2 \lambda\left(\mathrm{u}_{0} \mathrm{u}_{1}\right)\left[(\mathrm{uv}) \mathrm{v}_{1}\right],
\end{aligned}
$$

pois $\left(\mathrm{u}_{0} \mathrm{u}_{1}\right)\left\{\mathrm{u}_{0}\left[(\mathrm{uv}) \mathrm{v}_{1}\right]\right\} \in \mathrm{U}^{2}\{\mathrm{U}[(\mathrm{UV}) \mathrm{V}]\} \subseteq \mathrm{V}^{2}=0$. Da identidade (1.13) aplicada aos elementos $\mathrm{u}_{1}, \quad \mathrm{u}_{0}, \quad(\mathrm{uv}) \mathrm{v}_{1}, \quad$ vem $\mathrm{u}_{1}\left\{\mathrm{u}_{0}\left[(\mathrm{uv}) \mathrm{v}_{1}\right]\right\}+$ $+\mathrm{u}_{0}\left\{\mathrm{u}_{1}\left[(\mathrm{uv}) \mathrm{v}_{1}\right]\right\}+\left(\mathrm{u}_{0} \mathrm{u}_{1}\right)\left[(\mathrm{uv}) \mathrm{v}_{1}\right]=0$. Portanto $2 \lambda \mathrm{u}_{1}\left\{\mathrm{u}_{0}\left[(\mathrm{uv}) \mathrm{v}_{1}\right]\right\}+$ $+2 \lambda\left(\mathrm{u}_{0} \mathrm{u}_{1}\right)\left[(\mathrm{uv}) \mathrm{v}_{1}\right]=-2 \lambda \mathrm{u}_{0}\left\{\mathrm{u}_{1}\left[(\mathrm{uv}) \mathrm{v}_{1}\right]\right\}$ e assim segue o resultado.

Proposição $10 \quad O$ subespaço vetorial $\left[\left(U_{0} V_{0}\right) V_{0}\right] V_{0}$ é gerado pelos elementos da forma $\left[(u v) v_{1}\right] v_{2}+2 \lambda^{2} u_{0}^{2}\left\{\left[(u v) v_{1}\right] v_{2}\right\} \oplus 2 \lambda u_{0}\left\{\left[(u v) v_{1}\right] v_{2}\right\}$, com $u \in U$ e $v, v_{1}, v_{2} \in V$.

Prova: Pela Proposição 3 e por (1.21), um elemento $y$ do subespaço $\left[\left(\mathrm{U}_{0} \mathrm{~V}_{0}\right) \mathrm{V}_{0}\right] \mathrm{V}_{0}$ é da forma

$$
\begin{aligned}
\mathrm{y} & =\left\{(\mathrm{uv}) \mathrm{v}_{1}+2 \lambda \mathrm{u}_{0}\left[(\mathrm{uv}) \mathrm{v}_{1}\right]\right\}\left(\mathrm{v}_{2}-2 \lambda \mathrm{u}_{0} \mathrm{v}_{2}\right)= \\
& =\left[(\mathrm{uv}) \mathrm{v}_{1}\right] \mathrm{v}_{2}-2 \lambda\left(\mathrm{u}_{0} \mathrm{v}_{2}\right)\left[(\mathrm{uv}) \mathrm{v}_{1}\right]-4 \lambda^{2}\left(\mathrm{u}_{0} \mathrm{v}_{2}\right)\left\{\mathrm{u}_{0}\left[(\mathrm{uv}) \mathrm{v}_{1}\right]\right\},
\end{aligned}
$$

pois $\left\{\mathrm{u}_{0}\left[(\mathrm{uv}) \mathrm{v}_{1}\right]\right\} \mathrm{v}_{2} \in\{\mathrm{U}[(\mathrm{UV}) \mathrm{V}]\} \mathrm{V} \subseteq \mathrm{V}^{2}=0$. Da identidade (4.4) e de $u_{0},(u v) v_{1} \in U$, segue que $\left(u_{0} v_{2}\right)\left[(u v) v_{1}\right]=-u_{0}\left\{\left[(u v) v_{1}\right] v_{2}\right\}$. Além disso, como $v_{2}, u_{0}\left[(u v) v_{1}\right] \in V$, aplicando sucessivamente as identidades (4.3), (4.1) e (4.3), temos $2\left(\mathrm{u}_{0} \mathrm{v}_{2}\right)\left\{\mathrm{u}_{0}\left[(\mathrm{uv}) \mathrm{v}_{1}\right]\right\}=-2\left\{\mathrm{u}_{0}\left\{\mathrm{u}_{0}\left[(\mathrm{uv}) \mathrm{v}_{1}\right]\right\}\right\} \mathrm{v}_{2}=$ $=\left\{\mathrm{u}_{0}^{2}\left[(\mathrm{uv}) \mathrm{v}_{1}\right\} \mathrm{v}_{2}=-\mathrm{u}_{0}^{2}\left\{\left[(\mathrm{uv}) \mathrm{v}_{1}\right] \mathrm{v}_{2}\right\}\right.$. Substituindo na expresão de $y$. obtemos o resultado enunciado.

O próximo lema será utilizado repetidas vezes na continuação deste trabalho. Na sua demonstração, precisaremos utilizar os geradores dos P-monômios $\mathrm{m}\left(\mathrm{U}_{0}, \mathrm{~V}_{0}\right)$ calculados nas Proposições 1 a 10 acima.

Lema 5.1 Com as notações anteriores, temos as seguintes inclusões:

$$
\begin{gathered}
U_{0} V_{0} \subseteq U V \oplus U(U V) \\
U_{0}\left(U_{0} V_{0}\right) \subseteq U[U(U V)] \ominus U(U V)
\end{gathered}
$$




$$
\begin{gathered}
\left(U_{0} V_{0}\right) V_{0} \subseteq(U V) V \oplus U[(U V) V] \\
U_{0}^{3} V_{0} \subseteq U^{3} V \oplus U\left(U^{3} V\right) \\
U_{0}\left[U_{0}\left(U_{0} V_{0}\right)\right] \subseteq U[U(U V)] \oplus U\{U[U(U V)]\} \\
U_{0}\left[\left(U_{0} V_{0}\right) V_{0}\right] \subseteq U(U V)^{2} \oplus U[(U V) V] \\
{\left[\left(U_{0} V_{0}\right) V_{0}\right] V_{0} \subseteq[(U V) V] V \oplus U\{[(U V) V] V\}}
\end{gathered}
$$

Prova: Antes de iniciarmos as demonstrações, observemos que é suficiente provar que os geradores dos subespaços à esquerda em (5.1), (5.2), ... (5.7) estão nos subespaços da direita.

(5.1) Como foi estabelecido na Proposição 1 , um gerador $y$ de $\mathrm{U}_{0} \mathrm{~V}_{0}$ é da forma $\mathrm{y}=\left[\mathrm{uv}+2 \lambda^{2} \mathrm{u}_{0}^{2}(\mathrm{uv})\right] \oplus 2 \lambda \mathrm{u}_{0}(\mathrm{uv}) \in\left[\mathrm{UV}+\mathrm{U}^{2}(\mathrm{UV})\right] \oplus$ $\oplus \mathrm{U}(\mathrm{UV})$ e como $\mathrm{UV} \subseteq \mathrm{U}, \mathrm{U}^{2} \subseteq \mathrm{V}$, segue que $\mathrm{U}^{2}(\mathrm{UV}) \subseteq \mathrm{VU}=\mathrm{UV}$ logo $\mathrm{y} \in \mathrm{UV} \oplus \mathrm{U}(\mathrm{UV})$.

(5.2) Vimos na Proposição 2 que um gerador de $\mathrm{U}_{0}\left(\mathrm{U}_{0} \mathrm{~V}_{0}\right)$ é da forma y $=$ $=\left\{-2 \lambda \mathrm{u}_{0}\left[\mathrm{u}_{1}(\mathrm{uv})\right]+4 \lambda^{3} \mathrm{u}_{0}\left[\left(\mathrm{u}_{1} \mathrm{u}_{0}^{2}\right)(\mathrm{uv})\right]\right\} \oplus\left\{\mathrm{u}_{1}(\mathrm{uv})-2 \lambda^{2}\left(\mathrm{u}_{1} \mathrm{u}_{0}^{2}\right)(\mathrm{uv})\right\} \in$ $\in \mathrm{U}[\mathrm{U}(\mathrm{UV})]+\mathrm{U}\left[\mathrm{U}^{3}(\mathrm{UV})\right] \oplus\left\{\mathrm{U}(\mathrm{UV})+\mathrm{U}^{3}(\mathrm{UV})\right\} \subseteq\{\mathrm{U}[\mathrm{U}(\mathrm{UV})]+$ $+\mathrm{U}[\mathrm{U}(\mathrm{UV})]\} \oplus\{\mathrm{U}(\mathrm{UV})+\mathrm{U}(\mathrm{UV})\}=\mathrm{U}[\mathrm{U}(\mathrm{UV})] \oplus \mathrm{U}(\mathrm{UV})$ e assim segue que $\mathrm{y} \in \mathrm{U}[\mathrm{U}(\mathrm{UV})] \oplus \mathrm{U}(\mathrm{UV})$.

(5.3) Pela Proposição 3, um gerador $y$ de $\left(\mathrm{U}_{0} \mathrm{~V}_{0}\right) \mathrm{V}_{0}$ é da forma $\mathrm{y}=$ $=(\mathrm{uv}) \mathrm{v}_{1} \oplus 2 \lambda \mathrm{u}_{0}\left[(\mathrm{uv}) \mathrm{v}_{1}\right] \in(\mathrm{UV}) \mathrm{V} \oplus \mathrm{U}[(\mathrm{UV}) \mathrm{V}]$.

(5.4) Na Proposição 7 vimos que um gerador $y$ de $\mathrm{U}_{0}^{3} \mathrm{~V}_{0}$ é da forma $\mathrm{y}=\left[\mathrm{u}_{2}\left(\mathrm{uu}_{1}\right)\right] \mathrm{v} \oplus 2 \lambda \mathrm{u}_{0}\left\{\left[\mathrm{u}_{2}\left(\mathrm{uu}_{1}\right)\right] \mathrm{v}\right\} \in \mathrm{U}^{3} \mathrm{~V} \oplus \mathrm{U}\left(\mathrm{U}^{3} \mathrm{~V}\right)$.

(5.5) Vimos na Proposição 8 que um gerador do subespaço $\mathrm{U}_{0}\left[\mathrm{U}_{0}\left(\mathrm{U}_{0} \mathrm{~V}_{0}\right)\right]$ é da forma $\mathrm{y}=\mathrm{u}_{2}\left[\mathrm{u}_{1}(\mathrm{uv})\right]-4 \lambda^{2} \mathrm{u}_{2}\left\{\mathrm{u}_{0}\left[\left(\mathrm{u}_{0} \mathrm{u}_{1}\right)(\mathrm{uv})\right]\right\}+2 \lambda^{2} \mathrm{u}_{0}^{2}\left\{\mathrm{u}_{2}\left[\mathrm{u}_{1}(\mathrm{uv})\right]\right\}+$ $+8 \lambda^{4}\left(\mathrm{u}_{0} \mathrm{u}_{2}\right)\left\{\mathrm{u}_{0}\left[\left(\mathrm{u}_{1} \mathrm{u}_{0}^{2}\right)(\mathrm{uv})\right]\right\} \oplus 2 \lambda \mathrm{u}_{0}\left\{\mathrm{u}_{2}\left[\mathrm{u}_{1}(\mathrm{uv})\right]\right\}-4 \lambda^{3} \mathrm{u}_{0}\left\{\mathrm{u}_{2}\left[\left(\mathrm{u}_{1} \mathrm{u}_{0}^{2}\right)(\mathrm{uv})\right]\right\} \in$ $\in \mathrm{U}[\mathrm{U}(\mathrm{UV})]+\mathrm{U}\left\{\mathrm{U}\left[\mathrm{U}^{2}(\mathrm{UV})\right]\right\}+\mathrm{U}^{2}\{\mathrm{U}[\mathrm{U}(\mathrm{UV})]\}+\mathrm{U}^{2}\left\{\mathrm{U}\left[\mathrm{U}^{3}(\mathrm{UV})\right]\right\} \ominus$ $\oplus \mathrm{U}\{\mathrm{U}[\mathrm{U}(\mathrm{UV})]\}+\mathrm{U}\left\{\mathrm{U}\left[\mathrm{U}^{3}(\mathrm{UV})\right]\right\} \subseteq \mathrm{U}[\mathrm{U}(\mathrm{UV})]+\mathrm{U}\left\{\mathrm{U}\left[\mathrm{U}^{2}(\mathrm{UV})\right]\right\}+$ $+\mathrm{U}^{2}\{\mathrm{U}[\mathrm{U}(\mathrm{UV})]\} \oplus \mathrm{U}\{\mathrm{U}[\mathrm{U}(\mathrm{UV})]\}$, pois $\mathrm{U}^{3} \subseteq \mathrm{U}$. Mas: 


$$
\begin{gathered}
\mathrm{U}\left\{\mathrm{U}\left[\mathrm{U}^{2}(\mathrm{UV})\right]\right\} \subseteq \mathrm{U}\{\mathrm{U}[\mathrm{V}(\mathrm{UV})]\} \subseteq \mathrm{U}[\mathrm{U}(\mathrm{UV})]: \\
\mathrm{U}^{2}\{\mathrm{U}[\mathrm{U}(\mathrm{UV})]\} \subseteq \mathrm{U}^{2}\left[\mathrm{UU}^{2}\right] \subseteq \mathrm{U}^{2}(\mathrm{UV})=\mathrm{U}^{3} \mathrm{~V} \subseteq \mathrm{U}[\mathrm{U}(\mathrm{UV})] ;
\end{gathered}
$$

Portanto, $\mathrm{y} \in \mathrm{U}[\mathrm{U}(\mathrm{UV})] \oplus \mathrm{U}\{\mathrm{U}[\mathrm{U}(\mathrm{UV})]\}$.

(5.6) Como foi estabelecido pela Proposição 9, um gerador $y$ do subespaço $\mathrm{U}_{0}\left[\left(\mathrm{U}_{0} \mathrm{~V}_{0}\right) \mathrm{V}_{0}\right]$ é da forma $-2 \lambda \mathrm{u}_{0}\left\{\mathrm{u}_{1}\left[(\mathrm{uv}) \mathrm{v}_{1}\right]\right\} \oplus \mathrm{u}_{1}\left[(\mathrm{uv}) \mathrm{v}_{1}\right] \in$ $\in \mathrm{U}\{\mathrm{U}[(\mathrm{UV}) \mathrm{V}]\} \oplus \mathrm{U}[(\mathrm{UV}) \mathrm{V}]$ e como pelo Lema $3.1 \mathrm{U}[(\mathrm{UV}) \mathrm{V}]=(\mathrm{UV})^{2}$, segue que $y \in U(U V)^{2} \oplus U[(U V) V]$.

(5.7) Finalmente, pela Proposição 10 , um gerador $y$ de $\left[\left(\mathrm{U}_{0} \mathrm{~V}_{0}\right) \mathrm{V}_{0}\right] \mathrm{V}_{0}$ é da forma $\mathrm{y}=\left[(\mathrm{uv}) \mathrm{v}_{1}\right] \mathrm{v}_{2}+2 \lambda^{2} \mathrm{u}_{0}^{2}\left\{\left[(\mathrm{uv}) \mathrm{v}_{1}\right] \mathrm{v}_{2}\right\} \oplus 2 \lambda \mathrm{u}_{0}\left\{\left[(\mathrm{uv}) \mathrm{v}_{1}\right] \mathrm{v}_{2}\right\} \in$ $\in[(\mathrm{UV}) \mathrm{V}] \mathrm{V}+\mathrm{U}^{2}\{[(\mathrm{UV}) \mathrm{V}] \mathrm{V}\} \oplus \mathrm{U}\{[(\mathrm{UV}) \mathrm{V}] \mathrm{V}\}$. Mas $\mathrm{U}^{2}\{[(\mathrm{UV}) \mathrm{V}] \mathrm{V}\} \subseteq$ $\subseteq \mathrm{V}\{[(\mathrm{UV}) \mathrm{V}] \mathrm{V}\}=\{[(\mathrm{UV}) \mathrm{V}] \mathrm{V}\} \mathrm{V} \subseteq[(\mathrm{UV}) \mathrm{V}] \mathrm{V}$, pois $\mathrm{UV} \subseteq \mathrm{U}$, e isto mostra que y $\in[(U V) V] V \oplus \mathrm{U}\{[(\mathrm{UV}) \mathrm{V}] \mathrm{V}\}$.

Outro lema que será útil é o seguinte:

Lema 5.2 Para a decomposição de Peirce $A=F e \oplus U \oplus V$, temos

$$
\begin{gathered}
U^{5} \subseteq U[U(U V)] \\
U\left(U^{3} V\right)=U^{3}(U V) \subseteq U[(U V) V] \\
U(U V)^{2} \subseteq U^{3} V \subseteq U[U(U V)]
\end{gathered}
$$

Prova: (5.8) Um gerador de $\mathrm{U}^{5}$ é da forma $\mathrm{y}=\mathrm{u}\left\{\mathrm{u}_{1}\left[\mathrm{u}_{2}\left(\mathrm{u}_{3} \mathrm{u}_{4}\right)\right]\right\} \in$ $\in \mathrm{U}\left\{\mathrm{U}\left[\mathrm{U} \mathrm{U}^{2}\right]\right\}$ e como $\mathrm{U}^{2} \subseteq \mathrm{V}$, segue que y $\in \mathrm{U}[\mathrm{U}(\mathrm{UV})]$.

(5.9) Um gerador $y$ do P-monômio $\mathrm{U}^{3}(\mathrm{UV})$ é do tipo $\mathrm{y}=\left[\left(\mathrm{u}_{1} \mathrm{u}_{2}\right) \mathrm{u}_{3}\right](\mathrm{uv})=$ $=-\mathrm{u}\left\{\left[\left(\mathrm{u}_{1} \mathrm{u}_{2}\right) \mathrm{u}_{3}\right] \mathrm{v}\right\}$, sendo esta última igualdade consequência da identidade (4.4). Logo, y $\in \mathrm{U}\left[\left(\mathrm{U}^{2} \mathrm{U}\right) \mathrm{V}\right] \subseteq \mathrm{U}[(\mathrm{UV}) \mathrm{V}]$. A igualdade dada em (5.9) é um caso particular do Lema 3.2, considerando em (ii) $\mathrm{m}(\mathrm{U}, \mathrm{V})=$ $=\mathrm{V}$ e $n=1$.

(5.10) Um elemento gerador $y$ de $U(U V)^{2}$ é da forma $y=u\left[\left(u_{1} v_{1}\right)\left(u_{2} v_{2}\right)\right]$. De $\mathrm{J}\left(\mathrm{u}, \mathrm{u}_{1} \mathrm{v}_{1}, \mathrm{u}_{2} \mathrm{v}_{2}\right)=0$, obtemos $\mathrm{y}+\left(\mathrm{u}_{1} \mathrm{v}_{1}\right)\left[\mathrm{u}\left(\mathrm{u}_{2} \mathrm{v}_{2}\right)\right]+\left(\mathrm{u}_{2} \mathrm{v}_{2}\right)\left[\mathrm{u}\left(\mathrm{u}_{1} \mathrm{v}_{1}\right]=\right.$ 
$=0$. Usando-se a identidade de Jacobi para os elementos $\mathrm{u}_{1}, \mathrm{v}_{1}, \mathrm{u}\left(\mathrm{u}_{2} \mathrm{v}_{2}\right)$

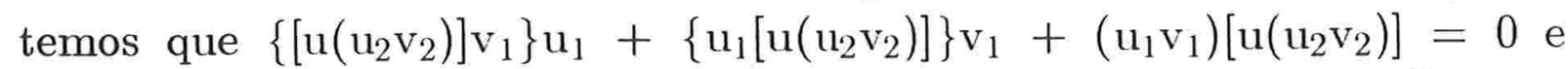
como $\left[\mathrm{u}\left(\mathrm{u}_{2} \mathrm{v}_{2}\right)\right] \mathrm{v}_{1}=0$, segue que $\left(\mathrm{u}_{1} \mathrm{v}_{1}\right)\left[\mathrm{u}\left(\mathrm{u}_{2} \mathrm{v}_{2}\right)\right]=-\left\{\mathrm{u}_{1}\left[\mathrm{u}\left(\mathrm{u}_{2} \mathrm{v}_{2}\right)\right]\right\} \mathrm{v}_{1} \in$

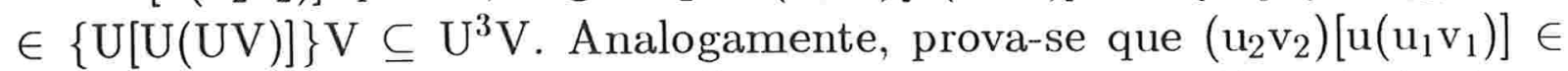
$\in \mathrm{U}^{3} \mathrm{~V}$, o que mostra que $\mathrm{y} \in \mathrm{U}^{3} \mathrm{~V}$. Finalmente, se $\mathrm{t}=\left[\left(\mathrm{u}_{1} \mathrm{u}_{2}\right) \mathrm{u}_{3}\right] \mathrm{v}$ é um gerador de $\mathrm{U}^{3} \mathrm{~V}$, então de $(4.3)$ e de $\mathrm{J}\left(\mathrm{u}_{1}, \mathrm{u}_{2}, \mathrm{u}_{3} \mathrm{v}\right)=0$, segue que $\mathrm{t}=$ $=-\left(\mathrm{u}_{3} \mathrm{v}\right)\left(\mathrm{u}_{1} \mathrm{u}_{2}\right)=\mathrm{u}_{1}\left[\mathrm{u}_{2}\left(\mathrm{u}_{3} \mathrm{v}\right)\right]+\mathrm{u}_{2}\left[\mathrm{u}_{1}\left(\mathrm{u}_{3} \mathrm{v}\right)\right] \in \mathrm{U}[\mathrm{U}(\mathrm{UV})]$.

\subsection{Invariância: Grupos I e II}

Podemos agora começar a estudar a invariância de P-subespaços em uma t-álgebra de posto 3 . O primeiro caso é:

Lema 5.3 O subespaço $\left\{U^{3}+(U V) V\right\} \oplus U^{2}$ é invariante por mudança de idempotentes, isto é, $\left\{U^{3}+(U V) V\right\} \oplus U^{2}=\left\{U_{0}^{3}+\left(U_{0} V_{0}\right) V_{0}\right\} \oplus U_{0}^{2}$.

Prova: Pelo Lema 2.6, temos a igualdade de ideais $\mathrm{U}_{0}^{3} \oplus \mathrm{U}_{0}^{2}=\mathrm{U}^{3} \oplus$ $\oplus \mathrm{U}^{2}$ e de (5.3) segue que $\left(\mathrm{U}_{0} \mathrm{~V}_{0}\right) \mathrm{V}_{0} \subseteq(\mathrm{UV}) \mathrm{V} \oplus \mathrm{U}[(\mathrm{UV}) \mathrm{V}] \subseteq(\mathrm{UV}) \mathrm{V} \oplus$ $\oplus \mathrm{U}(\mathrm{UV}) \subseteq(\mathrm{UV}) \mathrm{V} \oplus \mathrm{U}^{2}$. Assim $\left\{\mathrm{U}_{0}^{3}+\left(\mathrm{U}_{0} \mathrm{~V}_{0}\right) \mathrm{V}_{0}\right\} \oplus \mathrm{U}_{0}^{2}=\left[\mathrm{U}_{0}^{3} \oplus\right.$ $\left.\oplus \mathrm{U}_{0}^{2}\right]+\left(\mathrm{U}_{0} \mathrm{~V}_{0}\right) \mathrm{V}_{0} \subseteq\left[\mathrm{U}^{3} \oplus \mathrm{U}^{2}\right]+\left[(\mathrm{UV}) \mathrm{V} \oplus \mathrm{U}^{2}\right]=\left[\mathrm{U}^{3}+(\mathrm{UV}) \mathrm{V}\right] \oplus$ $\oplus \mathrm{U}^{2}$. Por simetria obtém-se a inclusão contrária.

Corolário 5.3.1 Para toda t-álgebra de posto 3, $A=F e \oplus U \oplus V$, a dimensão do subespaço $U^{3}+(U V) V$, contido em $U$, é invariante.

Prova: Segue de que $\operatorname{dim} \mathrm{U}^{2}$ é invariante por mudança de idempotentes.

Corolário 5.3.2 Os subespaços vetoriais $\left\{U^{3}+(U V) V\right\} \oplus V e$ $\left\{U^{3}+(U V) V\right\} \odot U^{2 n}$ têm dimensẫo invariante.

Prova: Segue do Corolário 5.3.1. de (1.23) e do Corolário 2.6.2. 
Lema 5.4 O subespaço $\left\{U^{3}+[(U V) V] V\right\} \oplus U^{2}$ da t-álgebra $A=$ $=F e \oplus U \oplus V$ é invariante por mudança de idempotentes.

Prova: Pelo Lema 2.6, temos $\mathrm{U}_{0}^{3} \oplus \mathrm{U}_{0}^{2}=\mathrm{U}^{3} \oplus \mathrm{U}^{2}$ e de (5.7) segue que $\left[\left(\mathrm{U}_{0} \mathrm{~V}_{0}\right) \mathrm{V}_{0}\right] \mathrm{V}_{0} \subseteq[(\mathrm{UV}) \mathrm{V}] \mathrm{V} \oplus \mathrm{U}\{[(\mathrm{UV}) \mathrm{V}] \mathrm{V}\} \subseteq[(\mathrm{UV}) \mathrm{V}] \mathrm{V} \oplus \mathrm{U}^{2}$, pois $[(\mathrm{UV}) \mathrm{V}] \mathrm{V} \subseteq \mathrm{U}$. Dessa forma, $\left\{\mathrm{U}_{0}^{3}+\left[\left(\mathrm{U}_{0} \mathrm{~V}_{0}\right) \mathrm{V}_{0}\right] \mathrm{V}_{0}\right\} \oplus \mathrm{U}_{0}^{2} \subseteq\left\{\mathrm{U}^{3}+\right.$ $+[(\mathrm{UV}) \mathrm{V}] \mathrm{V}\} \oplus \mathrm{U}^{2}$ e por simetria obtemos a inclusão contrária.

Corolário 5.4.1 A dimensão do subespaço vetorial $U^{3}+[(U V) V] V$, contido em $U$, de uma t-álgebra $A=F e \oplus U \oplus V$ é invariante por mudança de idempotentes.

Prova: Segue do fato que $\operatorname{dim} \mathrm{U}^{2}$ é invariante e do Lema 5.4.

Corolário 5.4.2 Os subespaços $\left\{U^{3}+[(U V) V] V\right\} \oplus \quad V$ e $\left\{U^{3}+\right.$ $+[(U V) V] V\} \oplus U^{2 n}$ têm dimensão invariante.

Prova: Segue do Corolário 5.4.1, de (1.23) e do Corolário 2.6.2.

Corolário 5.4.3 O subespaço $\left\{U^{3}+(U V) V\right\} \oplus\left\{U^{4}+U[(U V) V]\right\}$ é invariante por mudança de idempotentes.

Prova: Como, pelo Lema 2.6, $\mathrm{U}^{3} \oplus \mathrm{U}^{4}$ é invariante por mudança de idempotentes, basta mostrarmos que $\left(\mathrm{U}_{0} \mathrm{~V}_{0}\right) \mathrm{V}_{0} \oplus \mathrm{U}_{0}\left[\left(\mathrm{U}_{0} \mathrm{~V}_{0}\right) \mathrm{V}_{0}\right] \subseteq\left\{\mathrm{U}^{3}+\right.$ $+(\mathrm{UV}) \mathrm{V}\} \oplus\left\{\mathrm{U}^{4}+\mathrm{U}[(\mathrm{UV}) \mathrm{V}]\right\}$. Mas de (5.3), segue que $\left(\mathrm{U}_{0} \mathrm{~V}_{0}\right) \mathrm{V}_{0} \subseteq$ $\subseteq(\mathrm{UV}) \mathrm{V} \oplus \mathrm{U}[(\mathrm{UV}) \mathrm{V}]$ e de $(5.6)$, temos $\mathrm{U}_{0}\left[\left(\mathrm{U}_{0} \mathrm{~V}_{0}\right) \mathrm{V}_{0}\right] \subseteq \mathrm{U}(\mathrm{UV})^{2} \oplus$ $\oplus \mathrm{U}[(\mathrm{UV}) \mathrm{V}]$. Como de $(5.10), \mathrm{U}(\mathrm{UV})^{2} \subseteq \mathrm{U}^{3} \mathrm{~V}=\left(\mathrm{U}^{2} \mathrm{U}\right) \mathrm{V} \subseteq(\mathrm{UV}) \mathrm{V}$. obtemos a inclusão acima. Por simetria segue a inclusão contrária.

Corolário 5.4.4 Para toda t-álgebra $A=F e \oplus U \oplus V$, a dimensão do subespaço $U^{4}+U[(U V) V]$, que está contido em $V$, é invariante. 
Prova: Segue do fato que dim $\left[\mathrm{U}^{3}+(\mathrm{UV}) \mathrm{V}\right]$ é invariante por mudança de idempotentes e do Corolário 5.4.3.

Corolário 5.4.5 O subespaço $U^{3} \oplus\left\{U^{4}+U[(U V) V]\right\}$ é invariante por mudança de idempotentes.

Prova: Como no Corolário 5.4.4, basta mostrarmos que $\mathrm{U}_{0}\left[\left(\mathrm{U}_{0} \mathrm{~V}_{0}\right) \mathrm{V}_{0}\right] \subseteq$ $\subseteq \mathrm{U}^{3} \oplus\left\{\mathrm{U}^{4}+\mathrm{U}[(\mathrm{UV}) \mathrm{V}]\right\}$. Mas de (5.6) segue que $\mathrm{U}_{0}\left[\left(\mathrm{U}_{0} \mathrm{~V}_{0}\right) \mathrm{V}_{0}\right] \subseteq$ $\subseteq \mathrm{U}(\mathrm{UV})^{2} \oplus \mathrm{U}[(\mathrm{UV}) \mathrm{V}] \subseteq \mathrm{U}^{3} \oplus \mathrm{U}[(\mathrm{UV}) \mathrm{V}]$. A inclusão contrária é obtida por simetria.

Corolário 5.4.6 O subespaço $\left\{U^{3}+[(U V) V] V\right\} \oplus\left\{U^{4}+U[(U V) V]\right\}$ é invariante por mudança de idempotentes.

Prova: Usando-se o Corolário 5.4.5, resta apenas mostrarmos a inclusão $\left[\left(\mathrm{U}_{0} \mathrm{~V}_{0}\right) \mathrm{V}_{0}\right] \mathrm{V}_{0} \subseteq\left\{\mathrm{U}^{3}+[(\mathrm{UV}) \mathrm{V}] \mathrm{V}\right\} \oplus\left\{\mathrm{U}^{4}+\mathrm{U}[(\mathrm{UV}) \mathrm{V}]\right\}$. Mas de (5.7), temos $\left[\left(\mathrm{U}_{0} \mathrm{~V}_{0}\right) \mathrm{V}_{0}\right] \mathrm{V}_{0} \subseteq[(\mathrm{UV}) \mathrm{V}] \mathrm{V} \oplus \mathrm{U}\{[(\mathrm{UV}) \mathrm{V}] \mathrm{V}\}$ e como $\mathrm{UV} \subseteq \mathrm{U}$, segue a inclusão acima. Por simetria segue a inclusão contrária.

Corolário 5.4.7 Para toda t-álgebra $A=F e \oplus U \oplus V$, a dimensão do subespaço $U \ominus\left\{U^{4}+U[(U V) V]\right\}$ é invariante.

Prova: Segue do Corolário 5.4.4 e de (1.22).

Lema 5.5 Os seguintes subespaços da t-álgebra $A=F e \oplus U \oplus V$ são invariantes por mudança de idempotentes:

(i) $N^{2}=U V \oplus U^{2}$

(ii) $N^{3}=\left[U^{3}+(U V) V\right] \oplus U(U V)$

(iii) $N^{4}=\{U[U(U V)]+[(U V) V] V\} \oplus\left\{U^{4}+U[(U V) V]\right\}$ 
Prova: As igualdades acima são óbvias, pois $\mathrm{N}=\operatorname{ker} \omega$ independe da escolha de idempotentes.

Corolário 5.5.1 Com as mesmas hipóteses, os subespaços

$$
\begin{gathered}
U V \\
U(U V) \\
U[U(U V)]+[(U V) V] V
\end{gathered}
$$

têm dimensão invariante.

Prova: Os resultados seguem, respectivamente, do fato que as dimensões dos subespaços $U^{2}, U^{3}+(U V) V$ e $U^{4}+U[(U V) V]$ são invariantes por mudança de idempotentes.

Observação: A invariância da dimensão de UV pode ser provada também observando que o ideal de Etherington de A é dado por UV $\oplus \mathrm{V}$. Ver Corolário 5.5.3.

Corolário 5.5.2 Os seguintes subespaços de $A=F e \oplus U \oplus V$ têm dimensão invariante:

$$
\begin{gathered}
U V \oplus U^{4} \\
U V \oplus\left\{U^{4}+U[(U V) V]\right\} \\
U \oplus U(U V) \\
\{U[U(U V)]+[(U V) V] V\} \oplus V \\
\{U[U(U V)]+[(U V) V] V\} \oplus U^{2 n}
\end{gathered}
$$

Prova: Cada um dos resultados segue do Corolário 5.5.1 e respectivamente de: Corolário 2.6.2, Corolário 5.4.4, (1.22), (1.23) e novamente Corolário 2.6.2.

Corolário 5.5.3 Os seguintes subespaços são invariantes por mudança 
de idempotentes:
(i) $U V \oplus V$
(ideal de Etherington de A)
(ii) $U V \oplus U(U V)$
(iii) $U^{3} \oplus U(U V)$
(iv) $\left\{U^{3}+[(U V) V] V\right\} \oplus U(U V)$
(v) $\{U[U(U V)]+[(U V) V] V\} \oplus U(U V)$

Prova: (i) É conhecido que o ideal gerado pelos elementos $a^{2}-\omega(a) a$, com a $\in \mathrm{A}$, exprime-se por UV $\oplus \mathrm{V}$. Ver, por exemplo, [6].

(ii) De (5.1) temos $\mathrm{U}_{0} \mathrm{~V}_{0} \subseteq \mathrm{UV} \oplus \mathrm{U}(\mathrm{UV})$ e de (5.2) temos $\mathrm{U}_{0}\left(\mathrm{U}_{0} \mathrm{~V}_{0}\right) \subseteq$ $\subseteq \mathrm{U}[\mathrm{U}(\mathrm{UV})] \oplus \mathrm{U}(\mathrm{UV}) \subseteq \mathrm{UU}^{2} \oplus \mathrm{U}(\mathrm{UV}) \subseteq \mathrm{UV} \oplus \mathrm{U}(\mathrm{UV})$ e portanto obtemos a inclusão $\mathrm{U}_{0} \mathrm{~V}_{0} \oplus \mathrm{U}_{0}\left(\mathrm{U}_{0} \mathrm{~V}_{0}\right) \subseteq \mathrm{UV} \oplus \mathrm{U}(\mathrm{UV})$. A inclusão contrária vem por simetria.

(iii) Usando-se (5.2) e o Lema 2.6, respectivamente, temos $\mathrm{U}_{0}\left(\mathrm{U}_{0} \mathrm{~V}_{0}\right) \subseteq$ $\subseteq \mathrm{U}[\mathrm{U}(\mathrm{UV})] \oplus \mathrm{U}(\mathrm{UV})$ e $\mathrm{U}_{0}^{3} \subseteq \mathrm{U}^{3} \oplus \mathrm{U}^{4}$ e como $\mathrm{U}^{4} \subseteq \mathrm{U}(\mathrm{UV})$, segue que $\mathrm{U}_{0}^{3} \oplus \mathrm{U}_{0}\left(\mathrm{U}_{0} \mathrm{~V}_{0}\right) \subseteq\left\{\mathrm{U}^{3}+\mathrm{U}[\mathrm{U}(\mathrm{UV})]\right\} \oplus\left\{\mathrm{U}^{4}+\mathrm{U}(\mathrm{UV})\right\} \subseteq \mathrm{U}^{3} \odot$ $\oplus \mathrm{U}(\mathrm{UV})$. A inclusão contrária é obtida por simetria.

(iv) Usando-se o item (iii), resta apenas mostrarmos que $\left[\left(\mathrm{U}_{0} \mathrm{~V}_{0}\right) \mathrm{V}_{0}\right] \mathrm{V}_{0} \subseteq$ $\subseteq\left\{\mathrm{U}^{3}+[(\mathrm{UV}) \mathrm{V}] \mathrm{V}\right\} \oplus \mathrm{U}(\mathrm{UV})$. Mas como $(\mathrm{UV}) \mathrm{V} \subseteq \mathrm{U}$, de (5.7) temos $\left[\left(\mathrm{U}_{0} \mathrm{~V}_{0}\right) \mathrm{V}_{0}\right] \mathrm{V}_{0} \subseteq[(\mathrm{UV}) \mathrm{V}] \mathrm{V} \oplus \mathrm{U}\{[(\mathrm{UV}) \mathrm{V}] \mathrm{V}\} \subseteq[(\mathrm{UV}) \mathrm{V}] \mathrm{V} \oplus \mathrm{U}(\mathrm{UV})$, pois $(\mathrm{UV}) \mathrm{V} \subseteq \mathrm{U}$. Por simetria segue a inclusão contrária.

(v) De (5.5), temos $\mathrm{U}_{0}\left[\mathrm{U}_{0}\left(\mathrm{U}_{0} \mathrm{~V}_{0}\right)\right] \subseteq \mathrm{U}[\mathrm{U}(\mathrm{UV})] \oplus \mathrm{U}\{\mathrm{U}[\mathrm{U}(\mathrm{UV})]\}$ e como $\mathrm{U}(\mathrm{UV}) \subseteq \mathrm{V}$, segue que $\mathrm{U}_{0}\left[\mathrm{U}_{0}\left(\mathrm{U}_{0} \mathrm{~V}_{0}\right)\right] \subseteq \mathrm{U}[\mathrm{U}(\mathrm{UV})] \oplus \mathrm{U}(\mathrm{UV})$. De (5.7), temos $\left[\left(\mathrm{U}_{0} \mathrm{~V}_{0}\right) \mathrm{V}_{0}\right] \mathrm{V}_{0} \subseteq[(\mathrm{UV}) \mathrm{V}] \mathrm{V} \oplus \mathrm{U}\{[(\mathrm{UV}) \mathrm{V}] \mathrm{V}\}$ e como $(\mathrm{UV}) \mathrm{V} \subseteq \mathrm{U}$, segue que $\left[\left(\mathrm{U}_{0} \mathrm{~V}_{0}\right) \mathrm{V}_{0}\right] \mathrm{V}_{0} \subseteq[(\mathrm{UV}) \mathrm{V}] \mathrm{V} \odot \mathrm{U}(\mathrm{UV})$. Finalmente, de (5.2) temos $\mathrm{U}_{0}\left(\mathrm{U}_{0} \mathrm{~V}_{0}\right) \subseteq \mathrm{U}[\mathrm{U}(\mathrm{UV})] \oplus \mathrm{U}(\mathrm{UV})$ e com isso completamos a prova de que $\left\{\mathrm{U}_{0}\left[\mathrm{U}_{0}\left(\mathrm{U}_{0} \mathrm{~V}_{0}\right)\right]+\left[\left(\mathrm{U}_{0} \mathrm{~V}_{0}\right) \mathrm{V}_{0}\right] \mathrm{V}_{0}\right\} \oplus \mathrm{U}_{0}\left(\mathrm{U}_{0} \mathrm{~V}_{0}\right) \subseteq\{\mathrm{U}[\mathrm{U}(\mathrm{UV})]+$ $+\mathrm{U}[(\mathrm{UV}) \mathrm{V}]\} \in \mathrm{U}(\mathrm{UV})$. A inclusão contrária vem por simetria. 
Lema 5.6 O subespaço $U[U(U V)] \oplus U(U V)$ da t-álgebra $A=F e \oplus$ $\oplus U \oplus V$ é invariante por mudança de idempotentes.

Prova: De (5.2) e da prova do item (v) do Corolário 5.5.3, respectivamente, temos que $\mathrm{U}_{0}\left(\mathrm{U}_{0} \mathrm{~V}_{0}\right) \subseteq \mathrm{U}[\mathrm{U}(\mathrm{UV})] \oplus \mathrm{U}(\mathrm{UV})$ e $\mathrm{U}_{0}\left[\mathrm{U}_{0}\left(\mathrm{U}_{0} \mathrm{~V}_{0}\right)\right] \subseteq$ $\subseteq \mathrm{U}[\mathrm{U}(\mathrm{UV})] \oplus \mathrm{U}(\mathrm{UV})$ e portanto $\mathrm{U}_{0}\left[\mathrm{U}_{0}\left(\mathrm{U}_{0} \mathrm{~V}_{0}\right)\right] \oplus \mathrm{U}_{0}\left(\mathrm{U}_{0} \mathrm{~V}_{0}\right) \subseteq$ $\subseteq \mathrm{U}[\mathrm{U}(\mathrm{UV})] \oplus \mathrm{U}(\mathrm{UV})$. A inclusão contrária é obtida por simetria.

Corolário 5.6.1 A dimensão do subespaço vetorial $U[U(U V)]$, contido em $U$, da t-álgebra $A=F e \oplus U \oplus V$ é invariante.

Prova: Segue de que dim U(UV) é invariante, de acordo com Corolário 5.5.1.

Corolário 5.6.2 Os subespaços vetoriais

$$
\begin{gathered}
U[U(U V)] \oplus V \\
U[U(U V)] \oplus U^{2 n}
\end{gathered}
$$

têm dimensão invariante por mudança de idempotentes.

Pova: Os resultados seguem, respectivamente, do Corolário 5.6.1 e de (1.23) e Corolários 5.6.1 e 2.6.2.

Corolário 5.6.3 O subespaço vetorial $\{(U V) V+U[U(U V)]\} \oplus U(U V)$ de $A=F e \oplus U \oplus V$ é invariante por mudança de idempotentes.

Prova: Usando-se o resultado do Lema 5.6, resta apenas mostrarmos que $\left(\mathrm{U}_{0} \mathrm{~V}_{0}\right) \mathrm{V}_{0} \subseteq\{(\mathrm{UV}) \mathrm{V}+\mathrm{U}[\mathrm{U}(\mathrm{UV})] \oplus \mathrm{U}(\mathrm{UV})$. Mas de (5.3) e de $\mathrm{UV} \subseteq$ $\subseteq \mathrm{U}$, temos $\left(\mathrm{U}_{0} \mathrm{~V}_{0}\right) \mathrm{V}_{0} \subseteq(\mathrm{UV}) \mathrm{V} \oplus \mathrm{U}[(\mathrm{UV}) \mathrm{V}] \subseteq(\mathrm{UV}) \mathrm{V} \oplus \mathrm{U}(\mathrm{UV})$, e daí segue o resultado.

Corolário 5.6.4 O subespaço $(U V) V+U[U(U V)]$ de $A=F e \ominus U \Theta$ 
$\oplus \quad V$ tem dimensão invariante.

Prova: Segue do Corolário 5.6.3 e de que dim U(UV) é invariante por mudança de idempotentes.

Corolário 5.6.5 Os subespaços vetoriais

$$
\begin{gathered}
\{(U V) V+U[U(U V)]\} \oplus V \\
\{(U V) V+U[U(U V)]\} \oplus U^{2 n}
\end{gathered}
$$

têm dimensão invariante.

Prova: Os resultados seguem do Corolário 5.6.4 e respectivamente de (1.23) e Corolário 2.6.2.

Lema 5.7 O subespaço $U[U(U V)] \oplus U^{4}$ é invariante por mudança de idempotentes.

Prova: Do Lema 2.6 e de (5.8), temos $\mathrm{U}_{0}^{4} \subseteq \mathrm{U}^{5} \oplus \mathrm{U}^{4} \subseteq \mathrm{U}[\mathrm{U}(\mathrm{UV})] \oplus$ $\oplus \mathrm{U}^{4}$ e de (5.5), obtemos $\mathrm{U}_{0}\left[\mathrm{U}_{0}\left(\mathrm{U}_{0} \mathrm{~V}_{0}\right)\right] \subseteq \mathrm{U}[\mathrm{U}(\mathrm{UV})] \oplus \mathrm{U}\{\mathrm{U}[\mathrm{U}(\mathrm{UV})]\} \subseteq$ $\subseteq \mathrm{U}[\mathrm{U}(\mathrm{UV})] \oplus \mathrm{U}^{4}$. Portanto $\mathrm{U}_{0}\left[\mathrm{U}_{0}\left(\mathrm{U}_{0} \mathrm{~V}_{0}\right)\right] \oplus \mathrm{U}_{0}^{4}$ e por simetria segue a inclusão contrária.

Corolário 5.7.1 O subespaço $U[U(U V)] \oplus\left\{U^{4}+U[(U V) V]\right\} d a$ t-álgebra $A=F e \oplus U \oplus V$ é invariante por mudança de idempotentes.

Prova: Usando-se o resultado do Lema 5.7, resta apenas mostrarmos que $\mathrm{U}_{0}\left[\left(\mathrm{U}_{0} \mathrm{~V}_{0}\right) \mathrm{V}_{0}\right] \subseteq \mathrm{U}[\mathrm{U}(\mathrm{UV})] \oplus\left\{\mathrm{U}^{4}+\mathrm{U}[(\mathrm{UV}) \mathrm{V}]\right\}$. De (5.6), temos que $\mathrm{U}_{0}\left[\left(\mathrm{U}_{0} \mathrm{~V}_{0}\right) \mathrm{V}_{0}\right] \subseteq \mathrm{U}(\mathrm{UV})^{2} \oplus \mathrm{U}[(\mathrm{UV}) \mathrm{V}]$ e como de $(5.10), \mathrm{U}(\mathrm{UV})^{2} \subseteq$ $\subseteq \mathrm{U}^{3} \mathrm{~V} \subseteq \mathrm{U}[\mathrm{U}(\mathrm{UV})]$, segue que $\mathrm{U}_{0}\left[\mathrm{U}_{0}\left(\mathrm{U}_{0} \mathrm{~V}_{0}\right)\right] \ominus\left\{\mathrm{U}_{0}^{4}+\mathrm{U}_{0}\left[\left(\mathrm{U}_{0} \mathrm{~V}_{0}\right) \mathrm{V}_{0}\right]\right\} \subseteq$ $\subseteq \mathrm{U}[\mathrm{U}(\mathrm{UV})] \oplus\left\{\mathrm{U}^{4}+\mathrm{U}[(\mathrm{UV}) \mathrm{V}]\right\}$. Por simetria obtemos a inclusão contrária. 
Lema $5.8 O$ subespaço $(U V) V \oplus U[(U V) V]$ da tálgebra $A=F e \oplus$ $\oplus U \oplus V$ é invariante.

Prova: Das inclusões (5.6) e (5.10), temos $\mathrm{U}_{0}\left[\left(\mathrm{U}_{0} \mathrm{~V}_{0}\right) \mathrm{V}_{0}\right] \subseteq \mathrm{U}(\mathrm{UV})^{2} \oplus$ $\oplus \mathrm{U}[(\mathrm{UV}) \mathrm{V}] \subseteq \mathrm{U}^{3} \mathrm{~V} \oplus \mathrm{U}[(\mathrm{UV}) \mathrm{V}]=\left(\mathrm{UU}^{2}\right) \mathrm{V} \oplus \mathrm{U}[(\mathrm{UV}) \mathrm{V}] \subseteq(\mathrm{UV}) \mathrm{V} \oplus$ $\oplus \mathrm{U}[(\mathrm{UV}) \mathrm{V}]$. Além disso, de (5.3), obtemos $\left(\mathrm{U}_{0} \mathrm{~V}_{0}\right) \mathrm{V}_{0} \subseteq(\mathrm{UV}) \mathrm{V} \oplus$ $\oplus \mathrm{U}[(\mathrm{UV}) \mathrm{V}]$ e assim segue que $\left(\mathrm{U}_{0} \mathrm{~V}_{0}\right) \mathrm{V}_{0} \oplus \mathrm{U}_{0}\left[\left(\mathrm{U}_{0} \mathrm{~V}_{0}\right) \mathrm{V}_{0}\right] \subseteq(\mathrm{UV}) \mathrm{V} \oplus$ $\oplus \mathrm{U}[(\mathrm{UV}) \mathrm{V}]$. Por simetria obtemos a inclusão contrária.

Corolário $5.8 .1 \quad O$ subespaço vetorial $\{(U V) V+U[U(U V)]\} \oplus$ $\oplus\left\{U^{4}+U[(U V) V]\right\}$ da t-álgebra $A=F e \oplus U \oplus V$ é invariante.

Prova: Usando-se o resultado do Corolário 5.7.1, resta apenas mostrar$\operatorname{mos}$ que $\left(\mathrm{U}_{0} \mathrm{~V}_{0}\right) \mathrm{V}_{0} \subseteq\{(\mathrm{UV}) \mathrm{V}+\mathrm{U}[\mathrm{U}(\mathrm{UV})]\} \oplus\left\{\mathrm{U}^{4}+\mathrm{U}[(\mathrm{UV}) \mathrm{V}]\right\}$. Mas de (5.3) temos que $\left(\mathrm{U}_{0} \mathrm{~V}_{0}\right) \mathrm{V}_{0} \subseteq(\mathrm{UV}) \mathrm{V} \oplus \mathrm{U}[(\mathrm{UV}) \mathrm{V}]$, e daí segue que $\left\{\left(\mathrm{U}_{0} \mathrm{~V}_{0}\right) \mathrm{V}_{0}+\mathrm{U}_{0}\left[\mathrm{U}_{0}\left(\mathrm{U}_{0} \mathrm{~V}_{0}\right)\right]\right\} \oplus\left\{\mathrm{U}_{0}^{4}+\mathrm{U}_{0}\left[\left(\mathrm{U}_{0} \mathrm{~V}_{0}\right) \mathrm{V}_{0}\right]\right\} \subseteq\{(\mathrm{UV}) \mathrm{V}+$ $+\mathrm{U}[\mathrm{U}(\mathrm{UV})]\} \oplus\left\{\mathrm{U}^{4}+\mathrm{U}[(\mathrm{UV}) \mathrm{V}]\right\}$. Por simetria obtemos a inclusão contrária.

Lema 5.9 O subespaço vetorial $U^{3} V \oplus U[(U V) V]$ da t-álgebra de posto $3 A=F e \oplus U \oplus V$ é invariante.

Prova: De (5.4) e (5.9), temos $\mathrm{U}_{0}^{3} \mathrm{~V}_{0} \subseteq \mathrm{U}^{3} \mathrm{~V} \oplus \mathrm{U}[(\mathrm{UV}) \mathrm{V}]$. De (5.6) e (5.10), temos que $\mathrm{U}_{0}\left[\left(\mathrm{U}_{0} \mathrm{~V}_{0}\right) \mathrm{V}_{0}\right] \subseteq \mathrm{U}(\mathrm{UV})^{2} \oplus \mathrm{U}[(\mathrm{UV}) \mathrm{V}] \subseteq \mathrm{U}^{3} \mathrm{~V} \oplus$ $\oplus \mathrm{U}[(\mathrm{UV}) \mathrm{V}]$ e portanto $\mathrm{U}_{0}^{3} \mathrm{~V}_{0} \oplus \mathrm{U}_{0}\left[\left(\mathrm{U}_{0} \mathrm{~V}_{0}\right) \mathrm{V}_{0}\right] \subseteq \mathrm{U}^{3} \mathrm{~V} \oplus \mathrm{U}[(\mathrm{UV}) \mathrm{V}]$ e por simetria obtemos a inclusão contrária.

Corolário 5.9.1 O subespaço vetorial $\left\{U^{3} V+[(U V) V] V\right\} \ominus U[(U V) V]$ é invariante.

Prova: Usando-se o resultado do Lema 5.9, resta apenas mostrarmos que $\left[\left(\mathrm{U}_{0} \mathrm{~V}_{0}\right) \mathrm{V}_{0}\right] \mathrm{V}_{0} \subseteq\left\{\mathrm{U}^{3} \mathrm{~V}+[(\mathrm{UV}) \mathrm{V}] \mathrm{V}\right\} \oplus \mathrm{U}[(\mathrm{UV}) \mathrm{V}]$. Mas de (5.7), 
temos que $\left[\left(\mathrm{U}_{0} \mathrm{~V}_{0}\right) \mathrm{V}_{0}\right] \mathrm{V}_{0} \subseteq[(\mathrm{UV}) \mathrm{V}] \mathrm{V} \oplus \mathrm{U}\{[(\mathrm{UV}) \mathrm{V}] \mathrm{V}\} \subseteq[(\mathrm{UV}) \mathrm{V}] \mathrm{V} \in$ $\oplus \mathrm{U}[(\mathrm{UV}) \mathrm{V}]$, uma vez que $\mathrm{UV} \subseteq \mathrm{U}$. Portanto, $\left\{\mathrm{U}_{0}^{3} \mathrm{~V}_{0}+\left[\left(\mathrm{U}_{0} \mathrm{~V}_{0}\right) \mathrm{V}_{0}\right] \mathrm{V}_{0} \Theta\right.$ $\oplus \mathrm{U}_{0}\left[\left(\mathrm{U}_{0} \mathrm{~V}_{0}\right) \mathrm{V}_{0}\right] \subseteq\left\{\mathrm{U}^{3} \mathrm{~V}+[(\mathrm{UV}) \mathrm{V}] \mathrm{V}\right\} \oplus \mathrm{U}[(\mathrm{UV}) \mathrm{V}]$ e por simetria segue a inclusão contrária.

Lema 5.10 $O$ subespaço vetorial $(U V) V$ de $A=F e \oplus U \oplus V$ tem. dimensão invariante em relaçẫo às mudanç̧as de idempotentes em A.

Prova: Tomemos o idempotente $\mathrm{e}_{0}=\mathrm{e}+\mathrm{u}_{0}+\lambda \mathrm{u}_{0}^{2}$, com $\mathrm{u}_{0} \in \mathrm{U}$. Observemos antes que se $\mathrm{x} \in(\mathrm{UV}) \mathrm{V}$ então $\mathrm{x}+2 \lambda_{\mathrm{u}} \mathrm{x} \in\left(\mathrm{U}_{0} \mathrm{~V}_{0}\right) \mathrm{V}_{0}$. De fato, se $\mathrm{x} \in(\mathrm{UV}) \mathrm{V}$ então $x$ é uma soma finita de produtos $\left(\mathrm{u}^{i} \mathrm{v}^{i}\right) \mathrm{v}_{1}^{i}$, com $\mathrm{u}^{i} \in$ $\mathrm{U}$ e $\mathrm{v}^{i}, \mathrm{v}_{1}^{i} \in \mathrm{V}$, isto é, $\mathrm{x}=\sum_{i}\left(\mathrm{u}^{i} \mathrm{v}^{i}\right) \mathrm{v}_{1}^{i}$. Então

$$
\begin{aligned}
& \mathrm{x}+2 \lambda \mathrm{u}_{0} \mathrm{x}=\sum_{i}\left(\mathrm{u}^{i} \mathrm{v}^{i}\right) \mathrm{v}_{1}^{i}+2 \lambda \sum_{i}\left(\mathrm{u}^{i} \mathrm{v}^{i}\right) \mathrm{v}_{1}^{i}= \\
& =\sum_{i}\left\{\left(\mathrm{u}^{i} \mathrm{v}^{i}\right) \mathrm{v}_{1}^{i}+2 \lambda \mathrm{u}_{0}\left[\left(\mathrm{u}^{i} \mathrm{v}^{i}\right) \mathrm{v}_{1}^{i}\right]\right\} \in\left(\mathrm{U}_{0} \mathrm{~V}_{0}\right) \mathrm{V}_{0},
\end{aligned}
$$

pois $\left(\mathrm{U}_{0} \mathrm{~V}_{0}\right) \mathrm{V}_{0}$ é gerado pelos elementos da forma (uv) $\mathrm{v}^{\prime} \oplus 2 \lambda \mathrm{u}_{0}\left[(\mathrm{uv}) \mathrm{v}^{\prime}\right]$, de acordo com a Proposição 3.

Consideremos $\varphi: \mathrm{x} \in(\mathrm{UV}) \mathrm{V} \mapsto \varphi(\mathrm{x})=\mathrm{x} \oplus 2 \lambda \mathrm{u}_{0} \mathrm{x} \in\left(\mathrm{U}_{0} \mathrm{~V}_{0}\right) \mathrm{V}_{0}$. Ela é claramente linear, injetiva (pois a variável $x$ é "somando direto" em $\varphi(\mathrm{x}))$ e sobrejetora, ainda pela Proposição 3 . Assim $\varphi$ é bijetora e resulta que $(\mathrm{UV}) \mathrm{V}$ e $\left(\mathrm{U}_{0} \mathrm{~V}_{0}\right) \mathrm{V}_{0}$ têm a mesma dimensão.

Corolário 5.10.1 Os seguintes subespaços de $A=F e \oplus U \oplus V$ têm dimensão invariante por mudança de idempotentes:

$$
\begin{gathered}
(U V) V \oplus V \\
(U V) V \oplus U(U V) \\
(U V) V \oplus U^{2 n} \\
(U V) V \oplus\left\{U^{4}+U[(U V) V]\right\}
\end{gathered}
$$

Prova: Os resultados são consequência do Lema 5.10 e respectivamente de (1.23), Corolário 5.5.1, Corolário 2.6.2 e Corolário 5.4.4. 
Lema 5.11 O subespaço vetorial $U[(U V) V]$ de $A=F e \oplus U \oplus V$ tem. dimensão invariante por mudança de idempotentes em $A$.

Prova: Fixemos o idempotente $\mathrm{e}_{0}=\mathrm{e}+\mathrm{u}_{0}+\lambda \mathrm{u}_{0}^{2}$, com $\mathrm{u}_{0} \in \mathrm{U}$. Se $\mathrm{x} \in \mathrm{U}[(\mathrm{UV}) \mathrm{V}]$ então $\mathrm{x}-2 \lambda \mathrm{u}_{0} \mathrm{x} \in \mathrm{U}_{0}\left[\left(\mathrm{U}_{0} \mathrm{~V}_{0}\right) \mathrm{V}_{0}\right]$, pois nesse caso $x$ é uma soma finita de produtos $\mathrm{u}_{1}^{i}\left[\left(\mathrm{u}^{i} \mathrm{v}^{i}\right) \mathrm{v}_{1}^{i}\right.$, sendo $\mathrm{u}^{i}, \mathrm{u}_{1}^{i} \in \mathrm{U}$ e $\mathrm{v}^{i}, \mathrm{v}_{1}^{i} \in \mathrm{V}$, isto é, $\mathrm{x}=\sum_{i} \mathrm{u}_{1}^{i}\left[\left(\mathrm{u}^{i} \mathrm{v}^{i}\right) \mathrm{v}_{1}^{i}\right]$. Dessa forma,

$$
\begin{aligned}
\mathrm{x}-2 \lambda \mathrm{u}_{0} \mathrm{x} & =\sum_{i} \mathrm{u}_{1}^{i}\left[\left(\mathrm{u}^{i} \mathrm{v}^{i}\right) \mathrm{v}_{1}^{i}\right]-2 \lambda \mathrm{u}_{0}\left\{\sum_{i} \mathrm{u}_{1}^{i}\left[\left(\mathrm{u}^{i} \mathrm{v}^{i}\right) \mathrm{v}_{1}^{i}\right]\right\}== \\
& =\sum_{i} \mathrm{u}_{1}^{i}\left[\left(\mathrm{u}^{i} \mathrm{v}^{i}\right) \mathrm{v}_{1}^{i}\right]-2 \lambda \mathrm{u}_{0}\left\{\mathrm{u}_{1}^{i}\left[\left(\mathrm{u}^{i} \mathrm{v}^{i}\right) \mathrm{v}_{1}^{i}\right]\right\} \in \mathrm{U}_{0}\left[\left(\mathrm{U}_{0} \mathrm{~V}_{0}\right) \mathrm{V}_{0}\right]
\end{aligned}
$$

pois pela Proposição $9, \mathrm{U}_{0}\left[\left(\mathrm{U}_{0} \mathrm{~V}_{0}\right) \mathrm{V}_{0}\right]$ é gerado pelos elementos da forma $-2 \lambda \mathrm{u}_{0}\left\{\mathrm{u}_{1}\left[(\mathrm{uv}) \mathrm{v}_{1}\right\} \oplus \mathrm{u}_{1}\left[(\mathrm{uv}) \mathrm{v}_{1}\right]\right.$.

Consideremos a função $\varphi: \mathrm{x} \in \mathrm{U}[(\mathrm{UV}) \mathrm{V}] \mapsto \varphi(\mathrm{x})=\mathrm{x}-2 \lambda \mathrm{u}_{0} \mathrm{x} \in$ $\in \mathrm{U}_{0}\left[\left(\mathrm{U}_{0} \mathrm{~V}_{0}\right) \mathrm{V}_{0}\right]$. A função $\varphi$ é obviamente linear, injetora (pelo mesmo argumento usado no Corolário 5.10) e sobrejetora (pela Proposição 9). Assim $\varphi$ é bijetora, portanto concluimos que $\mathrm{U}[(\mathrm{UV}) \mathrm{V}]$ e $\mathrm{U}_{0}\left[\left(\mathrm{U}_{0} \mathrm{~V}_{0}\right) \mathrm{V}_{0}\right]$ têm a mesma dimensão.

Corolário 5.11.1 Os seguintes subespaços de $A=F e \oplus U \oplus V$ têm. dimensẫo invariante por mudança de idempotentes:

$$
\begin{gathered}
U \ominus U[(U V) V] \\
U V \oplus U[(U V) V] \\
U^{3} \oplus U[(U V) V] \\
U[U(U V)] \oplus U[(U V) V] \\
{\left[U^{3}+(U V) V\right] \oplus U[(U V) V]} \\
\left\{U^{3}+[(U V) V] V\right\} \oplus U[(U V) V] \\
\{(U V) V+U[U(U V)]\} \oplus U[(U V) V] \\
\{U[U(U V)]+[(U V) V] V\} \oplus U[(U V) V]
\end{gathered}
$$

Prova: Os resultados acima são consequência do Lema 5.11 combinado. respectivamente. com (1.22) e Corolários 5.5.1. 2.6.2. 5.6.1, 5.3.1. 5.4.1. 
Corolário 5.11.2 O subespaço $U^{3} V+[(U V) V] V$ de $A=F e \oplus U \oplus$ $\oplus V$ tem dimensão invariante por mudança de idempotentes.

Prova: Do Corolário 5.9.1, o subespaço $\left\{\mathrm{U}^{3} \mathrm{~V}+[(\mathrm{UV}) \mathrm{V}] \mathrm{V}\right\} \oplus \mathrm{U}[(\mathrm{UV}) \mathrm{V}]$ é invariante por mudança de idempotentes e portanto sua dimensão é invariante. O resultado segue do Lema 5.11.

Corolário 5.11.3: Os seguintes subespaços de $A=F e \oplus U \oplus V$ têm dimensão invariante por mudança de idempotentes:

$$
\begin{gathered}
\left\{U^{3}+[(U V) V] V\right\} \oplus V \\
\left\{U^{3}+[(U V) V] V\right\} \oplus U(U V) \\
\left\{U^{3}+[(U V) V] V\right\} \oplus U^{2 n} \\
\left\{U^{3}+[(U V) V] V\right\} \oplus\left\{U^{4}+U[(U V) V]\right\}
\end{gathered}
$$

Prova: Os resultados acima seguem do Corolário 5.11 .2 combinado, respectivamente, com (1.23) e Corolários 5.5.1, 2.6.2 e 5.4.4.

Lema 5.12 O subespaço vetorial $U^{3} V$ de $A=F e \oplus U \oplus V$ tem dimensấo invariante por mudanças de idempotentes de $A$.

Prova: Fixados um idempotente $\mathrm{e}_{0}=\mathrm{e}+\mathrm{u}_{0}+\lambda \mathrm{u}_{0}^{2}$, com $\mathrm{u}_{0} \in \mathrm{U}$ e $\mathrm{x} \in \mathrm{U}^{3} \mathrm{~V}$, o elemento $\mathrm{x}+2 \lambda \mathrm{u}_{0} \mathrm{x} \in \mathrm{U}_{0}^{3} \mathrm{~V}_{0}$, pois $x$ é uma soma finita de produtos da forma $\left[\mathrm{u}_{2}^{i}\left(\mathrm{u}^{i} \mathrm{u}_{1}^{i}\right)\right] \mathrm{v}_{1}^{i}$, sendo $\mathrm{u}^{i}, \mathrm{u}_{1}^{i}, \mathrm{u}_{2}^{i} \in \mathrm{U}$ e $\mathrm{v}_{1}^{i} \in \mathrm{V}$; isto é, $\mathrm{x}=\sum_{i}\left[\mathrm{u}_{2}^{i}\left(\mathrm{u}^{i} \mathrm{u}_{1}^{i}\right)\right] \mathrm{v}_{1}^{i}$. Então:

$$
\begin{aligned}
& \mathrm{x}+2 \lambda \mathrm{u}_{0} \mathrm{x}=\sum_{i}\left[\mathrm{u}_{2}^{i}\left(\mathrm{u}^{i} \mathrm{u}_{1}^{i}\right)\right] \mathrm{v}_{1}^{i}+2 \lambda \mathrm{u}_{0}\left\{\sum_{i}\left[\mathrm{u}_{2}^{i}\left(\mathrm{u}^{i} \mathrm{u}_{1}^{i}\right)\right] \mathrm{v}_{1}^{i}\right\}= \\
& =\sum_{i}\left\{\left[\mathrm{u}_{2}^{i}\left(\mathrm{u}^{i} \mathrm{u}_{1}^{i}\right)\right] \mathrm{v}_{1}^{i}+2 \lambda \mathrm{u}_{0}\left\{\left[\mathrm{u}_{2}^{i}\left(\mathrm{u}^{i} \mathrm{u}_{1}^{i}\right)\right] \mathrm{v}_{1}^{i}\right\}\right\} \in \mathrm{U}_{0}^{3} \mathrm{~V}_{0},
\end{aligned}
$$

pois, de acordo com a Proposição $7, \mathrm{U}_{0}^{3} \mathrm{~V}_{0}$ é gerado pelos elementos da forma $\left[u_{2}\left(u_{1}\right)\right] v \ominus 2 \lambda u_{0}\left\{\left[u_{2}\left(u_{1}\right)\right] v\right\}$. 
Consideremos a função $\varphi: \mathrm{x} \in \mathrm{U}^{3} \mathrm{~V} \mapsto \varphi(\mathrm{x})=\mathrm{x}+2 \lambda \mathrm{u}_{0} \mathrm{x} \in \mathrm{U}_{0}^{3} \mathrm{~V}_{0}$. Esta função é claramente linear, injetora (pelo mesmo motivo visto no Lema 5.10) e sobrejetora (pela Proposição 7). Assim, $\varphi$ é bijetora e, portanto, $\mathrm{U}^{3} \mathrm{~V}$ e $\mathrm{U}_{0}^{3} \mathrm{~V}_{0}$ têm a mesma dimensão.

Corolário 5.12.1 Os seguintes subespaços de $A=F e \oplus U \oplus V$ têm dimensão invariante por mudança de idempotentes:

$$
\begin{gathered}
U^{3} V \oplus V \\
U^{3} V \oplus U(U V) \\
U^{3} V \oplus U^{2 n} \\
U^{3} V \oplus\left\{U^{4}+U[(U V) V]\right\}
\end{gathered}
$$

Prova: Os resultados acima são consequência do Lema 5.12 combinado, respectivamente, com (1.23) os Corolários 5.5.1, 2.6.2 e 5.4.4.

Lema 5.13 O subespaço vetorial $[(U V) V] V$ de $A=F e \oplus U \oplus V$ tem. dimensão invariante por mudança de idempotentes em $A$.

Prova: Tomemos o idempotente $\mathrm{e}_{0}=\mathrm{e}+\mathrm{u}_{0}+\lambda \mathrm{u}_{0}^{2}$, com $\mathrm{u}_{0} \in \mathrm{U}$. Se $\mathrm{x} \in$ $\in[(U V) V] V$, então $x+2 \lambda^{2} u_{0}^{2} x+2 \lambda u_{0} x \in\left[\left(U_{0} V_{0}\right) V_{0}\right] V_{0}$ pois, nesse caso, $x$ é uma soma finita de produtos $\left[\left(\mathrm{u}^{i} \mathrm{v}^{i}\right) \mathrm{v}_{1}^{i}\right] \mathrm{v}_{2}^{i}$, com $\mathrm{u}^{i} \in \mathrm{U} \mathrm{e} \mathrm{v}^{i}, \mathrm{v}_{1}^{i}, \mathrm{v}_{2}^{i} \in$ $\in \mathrm{V}$; isto é $\mathrm{x}=\sum_{i}\left[\left(\mathrm{u}^{i} \mathrm{v}^{i}\right) \mathrm{v}_{1}^{i}\right] \mathrm{v}_{2}^{i}$. Então:

$$
\begin{aligned}
& \mathrm{x}+2 \lambda^{2} \mathrm{u}_{0}^{2} \mathrm{x}+2 \lambda \mathrm{u}_{0} \mathrm{x}=\sum_{i}\left[\left(\mathrm{u}^{i} \mathrm{v}^{i}\right) \mathrm{v}_{1}^{i}\right] \mathrm{v}_{2}^{i}+2 \lambda^{2} \mathrm{u}_{0}^{2}\left\{\sum_{i}\left[\left(\mathrm{u}^{i} \mathrm{v}^{i}\right) \mathrm{v}_{1}^{i}\right] \mathrm{v}_{2}^{i}\right\}+ \\
&+2 \lambda \mathrm{u}_{0}\left\{\sum_{i}\left[\left(\mathrm{u}^{i} \mathrm{v}^{i}\right) \mathrm{v}_{1}^{i}\right] \mathrm{v}_{2}^{i}\right\}= \\
&=\sum_{i}\left\{\left[\left(\mathrm{u}^{i} \mathrm{v}^{i}\right) \mathrm{v}_{1}^{i}\right] \mathrm{v}_{2}^{i}+2 \lambda^{2} \mathrm{u}_{0}^{2}\left[\left(\mathrm{u}^{i} \mathrm{v}^{i}\right) \mathrm{v}_{1}^{i}\right] \mathrm{v}_{2}^{i}+2 \lambda \mathrm{u}_{0}\left[\left(\mathrm{u}^{i} \mathrm{v}^{i}\right) \mathrm{v}_{1}^{i}\right] \mathrm{v}_{2}^{i}\right\}
\end{aligned}
$$

é um elemento de $\left[\left(\mathrm{U}_{0} \mathrm{~V}_{0}\right) \mathrm{V}_{0}\right] \mathrm{V}_{0}$, pois, pela Proposição 10, este subespaço é gerado pelos elementos da forma $\left[(u v) v_{1}\right] v_{2}+2 \lambda^{2} u_{0}^{2}\left\{\left[(u v) v_{1}\right] v_{2}\right\} \oplus$ $\oplus 2 \lambda \mathrm{u}_{0}\left\{\left[(\mathrm{uv}) \mathrm{v}_{1}\right] \mathrm{v}_{2}\right\}$.

Consideremos a função $\varphi: \mathrm{x} \in[(\mathrm{UV}) \mathrm{V}] \mathrm{V} \mapsto \mathrm{x}+2 \lambda^{2} \mathrm{u}_{0}^{2} \mathrm{x}+2 \lambda \mathrm{u}_{0} \mathrm{x} \in$ $\in\left[\left(\mathrm{U}_{0} \mathrm{~V}_{0}\right) \mathrm{V}_{0}\right] \mathrm{V}_{0}$. A função $\varphi$ é obviamente linear e, pela Proposição 10 , 
ela é sobrejetora. Afirmamos que $\varphi$ é injetora. De fato, se $\mathrm{x}+2 \lambda^{2} \mathrm{u}_{0}^{2} \mathrm{x}+$ $+2 \lambda \mathrm{u}_{0} \mathrm{x}=\mathrm{y}+2 \lambda^{2} \mathrm{u}_{0}^{2} \mathrm{y}+2 \lambda \mathrm{u}_{0} \mathrm{y}$ então $(\mathrm{x}-\mathrm{y})+2 \lambda^{2} \mathrm{u}_{0}^{2}(\mathrm{x}-\mathrm{y})=$ $=2 \lambda \mathrm{u}_{0}(\mathrm{x}-\mathrm{y})$ e como $\mathrm{x}, \mathrm{y} \in[(\mathrm{UV}) \mathrm{V}] \mathrm{V} \subseteq \mathrm{U}$, segue que $\mathrm{u}_{0}^{2}(\mathrm{x}-\mathrm{y}) \in$ $\in \mathrm{U}$ e $\mathrm{u}_{0}(\mathrm{x}-\mathrm{y}) \in \mathrm{V}$ e portanto $(\mathrm{x}-\mathrm{y})+2 \lambda^{2} \mathrm{u}_{0}^{2}(\mathrm{x}-\mathrm{y})=2 \lambda \mathrm{u}_{0}(\mathrm{x}-\mathrm{y}) \in$ $\in \mathrm{U} \cap \mathrm{V}=0$. Ou seja, $\mathrm{u}_{0}(\mathrm{x}-\mathrm{y})=0$ e portanto $\mathrm{x}-\mathrm{y}=-2 \lambda^{2} \mathrm{u}_{0}^{2}(\mathrm{x}-\mathrm{y})=$ $=4 \lambda^{2} \mathrm{u}_{0}\left[\mathrm{u}_{0}(\mathrm{x}-\mathrm{y})\right]$, sendo esta última igualdade consequência de (4.1). Logo, $\mathrm{x}-\mathrm{y}=0$ e $\operatorname{assim} \mathrm{x}=\mathrm{y}$. Dessa forma, $\varphi$ é uma bijeção e daí concluimos que $[(\mathrm{UV}) \mathrm{V}] \mathrm{V}$ e $\left[\left(\mathrm{U}_{0} \mathrm{~V}_{0}\right) \mathrm{V}_{0}\right] \mathrm{V}_{0}$ têm a mesma dimensão.

Corolário 5.13.1 Os seguintes subespaços de $A=F e \oplus U \oplus V$ têm. dimensão invariante por mudança de idempotentes:

$$
\begin{gathered}
{[(U V) V] V \oplus V} \\
{[(U V) V] V \oplus U(U V)} \\
{[(U V) V] V \oplus U^{2 n}} \\
{[(U V) V] V \oplus U[(U V) V]} \\
{[(U V) V] V \oplus\left\{U^{4}+U[(U V) V]\right.}
\end{gathered}
$$

Prova: Os resultados acima seguem do Lema 5.13 combinado, respectivamente, com (1.23), Corolário 5.5.1, Corolário 2.6.2, Lema 5.11 e Corolário 5.4.4.

Lema 5.14 O subespaço $U^{3} V \oplus U^{3}(U V)$, de uma t-álgebra $A=F e \oplus$ $\oplus U \oplus V$ de posto 3, é invariante por mudança de idempotentes.

Prova: Devemos mostrar que $\mathrm{U}_{0}^{3} \mathrm{~V}_{0} \oplus \mathrm{U}_{0}^{3}\left(\mathrm{U}_{0} \mathrm{~V}_{0}\right)=\mathrm{U}^{3} \mathrm{~V} \oplus \mathrm{U}^{3}(\mathrm{UV})$. De (5.4) e de (5.9), temos $\mathrm{U}_{0}^{3} \mathrm{~V}_{0} \subseteq \mathrm{U}^{3} \mathrm{~V} \oplus \mathrm{U}\left(\mathrm{U}^{3} \mathrm{~V}\right)=\mathrm{U}^{3} \mathrm{~V} \oplus \mathrm{U}^{3}(\mathrm{UV})$. Por outro lado, de $(5.1)$ e de $\mathrm{U}_{0}^{3} \subseteq \mathrm{U}^{3} \oplus \mathrm{U}^{2}$ segue que $\mathrm{U}_{0}^{3}\left(\mathrm{U}_{0} \mathrm{~V}_{0}\right) \subseteq$ $\subseteq\left(\mathrm{U}^{3} \oplus \mathrm{U}^{2}\right)[\mathrm{UV} \oplus \mathrm{U}(\mathrm{UV})] \subseteq \mathrm{U}^{3}(\mathrm{UV})+\mathrm{U}^{3}[\mathrm{U}(\mathrm{UV})]+\mathrm{U}^{2}(\mathrm{UV})+$ $+\mathrm{U}^{2}[\mathrm{U}(\mathrm{UV})]=\left\{\mathrm{U}^{3}[\mathrm{U}(\mathrm{UV})]+\mathrm{U}^{2}(\mathrm{UV})\right\} \ominus \mathrm{U}^{3}(\mathrm{UV})=\left\{\mathrm{U}^{3}[\mathrm{U}(\mathrm{UV})]+\right.$ $\left.+U^{3} V\right\} \oplus U^{3}(U V)$, pois pelo Lema 3.1, $U^{2}(U V)=U^{3} V$. Além disso. $\mathrm{U}^{3}[\mathrm{U}(\mathrm{UV})] \subseteq \mathrm{U}^{3} \mathrm{U}^{2} \subseteq \mathrm{U}^{3} \mathrm{~V}$ e portanto $\mathrm{U}_{0}^{3}\left(\mathrm{U}_{0} \mathrm{~V}_{0}\right) \subseteq \mathrm{U}^{3} \mathrm{~V} \oplus \mathrm{U}^{3}(\mathrm{UV}) . \mathrm{A}$ inclusão contrária segue por simetria. 
Corolário 5.14.1 Em uma t-álgebra $A=F e \oplus U \oplus V$, de posto 3, a dimensão do subespaço $U^{3}(U V)$ contido em. $V$, é invariante.

Prova: Segue do fato de que $\operatorname{dim}\left(\mathrm{U}^{3} \mathrm{~V}\right)$ é invariante por mudança de idempotentes e do Lema 5.14.

Lema $\mathbf{5 . 1 5} O$ subespaço $U^{2 n+1} V \oplus U^{2 n+1}(U V)$, de uma t-álgebra $A=F e \oplus U \oplus V$ de posto 3, é invariante por mudança de idempotentes, para todo $n \geq 0$.

Prova: O Corolário 5.5.3 e o Lema 5.14 garantem que o resultado é verdadeiro para $n=0,1$, respectivamente. Para $n \geq 2$, devemos mostrar que $\mathrm{U}_{0}^{2 n+1} \mathrm{~V}_{0} \oplus \mathrm{U}_{0}^{2 n+1}\left(\mathrm{U}_{0} \mathrm{~V}_{0}\right)=\mathrm{U}^{2 n+1} \mathrm{~V} \oplus \mathrm{U}^{2 n+1}(\mathrm{UV})$. Um elemento gerador de $\mathrm{U}_{0}^{2 n+1} \mathrm{~V}_{0}$ é da forma $\mathrm{y}=\left[\mathrm{t}\left(\mathrm{u}+2 \lambda \mathrm{u}_{0} \mathrm{u}\right)\right]\left(\mathrm{v}-2 \lambda \mathrm{u}_{0} \mathrm{v}\right)$, com $\mathrm{t} \in \mathrm{U}_{0}^{2 n}$. Logo

$$
\begin{aligned}
\mathrm{y} & =\left[\mathrm{tu}+2 \lambda \mathrm{t}\left(\mathrm{u}_{0} \mathrm{u}\right)\right]\left(\mathrm{v}-2 \lambda \mathrm{u}_{0} \mathrm{v}\right)= \\
& =(\mathrm{tu}) \mathrm{v}-2 \lambda(\mathrm{tu})\left(\mathrm{u}_{0} \mathrm{v}\right)+2 \lambda\left[\mathrm{t}\left(\mathrm{u}_{0} \mathrm{u}\right)\right] \mathrm{v}-4 \lambda^{2}\left[\mathrm{t}\left(\mathrm{u}_{0} \mathrm{u}\right)\right]\left(\mathrm{u}_{0} \mathrm{v}\right)
\end{aligned}
$$

Portanto, $\mathrm{y} \in\left(\mathrm{U}_{0}^{2 n} \mathrm{U}\right) \mathrm{V}+\left(\mathrm{U}_{0}^{2 n} \mathrm{U}\right)(\mathrm{UV})+\left(\mathrm{U}_{0}^{2 n} \mathrm{U}^{2}\right) \mathrm{V}+\left(\mathrm{U}_{0}^{2 n} \mathrm{U}^{2}\right)(\mathrm{UV})$. Mas pelo Lema 2.6, $\mathrm{U}_{0}^{2 n} \mathrm{U} \subseteq\left(\mathrm{U}^{2 n} \oplus \mathrm{U}^{2 n-1}\right) \mathrm{U}=\mathrm{U}^{2 n+1} \oplus \mathrm{U}^{2 n}$. Além disso, ainda pelo Lema 2.6, $\mathrm{U}_{0}^{2 n} \mathrm{U}^{2} \subseteq\left(\mathrm{U}^{2 n} \oplus \mathrm{U}^{2 n+1}\right) \mathrm{U}^{2} \subseteq \mathrm{U}^{2 n} \mathrm{U}^{2} \oplus \mathrm{U}^{2 n-1} \mathrm{U}^{2}=$ $=\mathrm{U}^{2 n-1} \mathrm{U}^{2}$, pois $\mathrm{U}^{2 n} \mathrm{U}^{2} \subseteq \mathrm{V}^{2}=0$. Usando-se a identidade de Jacobi para os elementos $\mathrm{u}_{1} \in \mathrm{U}^{2 n-1}, \mathrm{u}_{2}, \mathrm{u}_{3} \in \mathrm{U}$, obtemos $\mathrm{u}_{1}\left(\mathrm{u}_{2} \mathrm{u}_{3}\right)=-\mathrm{u}_{2}\left(\mathrm{u}_{1} \mathrm{u}_{3}\right)+$ - $\mathrm{u}_{3}\left(\mathrm{u}_{1} \mathrm{u}_{2}\right)$, o que mostra que $\mathrm{U}^{2 n-1} \mathrm{U}^{2} \subseteq \mathrm{UU}^{2 n}=\mathrm{U}^{2 n+1}$ e portanto $\mathrm{U}_{0}^{2 n} \mathrm{U}^{2} \subseteq \mathrm{U}^{2 n+1}$. Dessa forma, temos

$$
\begin{aligned}
\mathrm{y} & \in\left(\mathrm{U}^{2 n+1} \oplus \mathrm{U}^{2 n}\right) \mathrm{V}+\left(\mathrm{U}^{2 n+1} \oplus \mathrm{U}^{2 n}\right)(\mathrm{UV})+\mathrm{U}^{2 n+1} \mathrm{~V}+\mathrm{U}^{2 n+1}(\mathrm{UV})= \\
& =\mathrm{U}^{2 n+1} \mathrm{~V}+\mathrm{U}^{2 n} \mathrm{~V}+\mathrm{U}^{2 n+1}(\mathrm{UV})+\mathrm{U}^{2 n}(\mathrm{UV})= \\
& =\mathrm{U}^{2 n+1} \mathrm{~V}+\mathrm{U}^{2 n+1}(\mathrm{UV})+\mathrm{U}^{2 n}(\mathrm{UV})= \\
& =\left[\mathrm{U}^{2 n}(\mathrm{UV})+\mathrm{U}^{2 n+1} \mathrm{~V}\right] \oplus \mathrm{U}^{2 n+1}(\mathrm{UV}),
\end{aligned}
$$

pois $\mathrm{U}^{2 n} \mathrm{~V} \subseteq \mathrm{V}^{2}=0$. Do Lema 3.2 , (ii), temos $\mathrm{U}^{2 n}(\mathrm{UV})=\mathrm{U}^{2 n+1} \mathrm{~V}$ e assim $\mathrm{U}_{0}^{2 n+1} \mathrm{~V}_{0} \subseteq \mathrm{U}^{2 n+1} \mathrm{~V} \oplus \mathrm{U}^{2 n+1}(\mathrm{UV})$. Consideremos, agora, um gerador $y$ 
do subespaço $\mathrm{U}_{0}^{2 n+1}\left(\mathrm{U}_{0} \mathrm{~V}_{0}\right)$. Então temos que $\mathrm{y}=\mathrm{x}\left[\mathrm{uv}+2 \lambda^{2} \mathrm{u}_{0}^{2}(\mathrm{uv})+\right.$ $\left.+2 \lambda \mathrm{u}_{0}(\mathrm{uv})\right], \mathrm{com} \mathrm{x} \in \mathrm{U}_{0}^{2 n+1}$. Logo, $\mathrm{y} \in \mathrm{U}_{0}^{2 n+1}\left[\mathrm{UV}+\mathrm{U}^{2}(\mathrm{UV})+\mathrm{U}(\mathrm{UV})\right] \subseteq$ $\subseteq\left(\mathrm{U}^{2 n+1} \oplus \mathrm{U}^{2 n}\right)[\mathrm{UV}+\mathrm{V}] \subseteq \mathrm{U}^{2 n+1}(\mathrm{UV})+\mathrm{U}^{2 n+1} \mathrm{~V}+\mathrm{U}^{2 n}(\mathrm{UV})$, sendo a primeira inclusão consequência do Lema 2.6 e de $\mathrm{U}^{2} \subseteq \mathrm{V}$ e UV $\subseteq \mathrm{U}$ e a última segue de $\mathrm{U}^{2 n} \mathrm{~V} \subseteq \mathrm{V}^{2}=0$. Novamente do Lema 3.2, (ii), temos $\mathrm{U}^{2 n}(\mathrm{UV})=\mathrm{U}^{2 n+1} \mathrm{~V}$, e portanto $\mathrm{U}_{0}^{2 n+1}\left(\mathrm{U}_{0} \mathrm{~V}_{0}\right) \subseteq \mathrm{U}^{2 n+1} \mathrm{~V} \oplus \mathrm{U}^{2 n+1}(\mathrm{UV})$, o que completa a prova de que $\mathrm{U}_{0}^{2 n+1} \mathrm{~V}_{0} \oplus \mathrm{U}_{0}^{2 n+1}\left(\mathrm{U}_{0} \mathrm{~V}_{0}\right)=\mathrm{U}^{2 n+1} \mathrm{~V} \oplus$ $\oplus \mathrm{U}^{2 n+1}(\mathrm{UV})$. A inclusão contrária é obtida por simetria.

Corolário 5.15.1 O subespaço $U^{2 n+1} V \oplus U^{2 n+1}(U V)$ tem dimensão invariante, para todo $n \geq 0$.

Corolário 5.15.2 Em uma t-álgebra de posto $3 A=F e \oplus U \oplus V$, as seguintes condições são equivalentes:

(i) o subespaço $U^{2 n+1} V$ tem dimensão invariante;

(ii) o subespaço $U^{2 n+1}(U V)$ tem dimensão invariante.

Após a demonstração de todos os resultados sobre a invariância que conseguimos, podemos dividir o conjunto dos 89 P-subespaços de grau $\leq$ 4 que aparecem no Apêndice nos Grupos I e II abaixo. Observemos que todos os 89 subespaços têm dimensão invariante.

GRUPO I: Neste Grupo listamos todos os subespaços $\mathrm{p}(\mathrm{U}, \mathrm{V})$ de grau $\leq 4$ que sabemos serem genericamente invariantes por mudança de idempotentes, isto é, aqueles para os quais temos $\mathrm{p}(\mathrm{U}, \mathrm{V})=\mathrm{p}\left(\mathrm{U}_{0}, \mathrm{~V}_{0}\right)$ para todo idempotente $e_{0}$ e para toda t-álgebra, de posto $3, \mathrm{~A}=\mathrm{Fe} \oplus \mathrm{U} \oplus$ V. Observemos que nenhum subespaço monomial aparece no Grupo I. 
13. $\mathrm{U} \oplus \mathrm{U}^{2}$

17. $\mathrm{UV} \oplus \mathrm{V}$

23. $\mathrm{UV} \oplus \mathrm{U}^{2}=\mathrm{N}^{2}$

24. $\mathrm{U}^{3} \oplus \mathrm{U}^{2}$

29. $\mathrm{UV} \oplus \mathrm{U}(\mathrm{UV})$

32. $\mathrm{U}^{3} \oplus \mathrm{U}(\mathrm{UV})$

34. $\mathrm{U}^{3} \oplus \mathrm{U}^{4}$

38. $\mathrm{U}[\mathrm{U}(\mathrm{UV})] \oplus \mathrm{U}(\mathrm{UV})$

43. $(\mathrm{UV}) \mathrm{V} \oplus \mathrm{U}[(\mathrm{UV}) \mathrm{V}]$

44. $\mathrm{U}[\mathrm{U}(\mathrm{UV})] \oplus \mathrm{U}^{4}$

50. $\mathrm{U}^{3} \mathrm{~V} \oplus \mathrm{U}[(\mathrm{UV}) \mathrm{V}]$

59. $\left[\mathrm{U}^{3}+(\mathrm{UV}) \mathrm{V}\right] \oplus \mathrm{U}^{2}$

60. ${ }^{\circ}\left\{\mathrm{U}^{3}+[(\mathrm{UV}) \mathrm{V}] \mathrm{V}\right\} \oplus \mathrm{U}^{2}$

65. $\left[\mathrm{U}^{3}+(\mathrm{UV}) \mathrm{V}\right] \oplus \mathrm{U}(\mathrm{UV})=\mathrm{N}^{3}$

66. $\left\{\mathrm{U}^{3}+[(\mathrm{UV}) \mathrm{V}] \mathrm{V}\right\} \oplus \mathrm{U}(\mathrm{UV})$

69. $\mathrm{U}^{3} \oplus\left\{\mathrm{U}^{4}+\mathrm{U}[(\mathrm{UV}) \mathrm{V}]\right\}$

72. $\{(\mathrm{UV}) \mathrm{V}+\mathrm{U}[\mathrm{U}(\mathrm{UV})]\} \oplus \mathrm{U}(\mathrm{UV})$

73. $\{\mathrm{U}[\mathrm{U}(\mathrm{UV})]+[(\mathrm{UV}) \mathrm{V}] \mathrm{V}\} \oplus \mathrm{U}(\mathrm{UV})$

78. $\mathrm{U}[\mathrm{U}(\mathrm{UV})] \oplus\left\{\mathrm{U}^{4}+\mathrm{U}[(\mathrm{UV}) \mathrm{V}]\right\}$

84. $\left\{\mathrm{U}^{3} \mathrm{~V}+[(\mathrm{UV}) \mathrm{V}] \mathrm{V}\right\} \oplus \mathrm{U}[(\mathrm{UV}) \mathrm{V}]$

85. $\left[\mathrm{U}^{3}+(\mathrm{UV}) \mathrm{V}\right] \oplus\left\{\mathrm{U}^{4}+\mathrm{U}[(\mathrm{UV}) \mathrm{V}]\right\}$

86. $\left\{\mathrm{U}^{3}+[(\mathrm{UV}) \mathrm{V}] \mathrm{V}\right\} \oplus\left\{\mathrm{U}^{4}+\mathrm{U}[(\mathrm{UV}) \mathrm{V}]\right\}$

87. $\left\{(\mathrm{UV}) \mathrm{V}+\mathrm{U}[\mathrm{U}(\mathrm{UV})] \oplus\left\{\mathrm{U}^{4}+\mathrm{U}[(\mathrm{UV}) \mathrm{V}]\right\}\right.$

88. $\{\mathrm{U}[\mathrm{U}(\mathrm{UV})]+[(\mathrm{UV}) \mathrm{V}] \mathrm{V}\} \oplus\left\{\mathrm{U}^{4}+\mathrm{U}[(\mathrm{UV}) \mathrm{V}]\right\}=\mathrm{N}^{4}$

GRUPO II: Neste Grupo, listamos todos os subespaços p(U, V) cujas dimensões são genericamente invariantes por mudança de idempotentes. isto é, aqueles para os quais $\operatorname{dim} \mathrm{p}(\mathrm{U}, \mathrm{V})=\operatorname{dim} \mathrm{p}\left(\mathrm{U}_{0}, \mathrm{~V}_{0}\right)$, para todo idempotente $e_{0}$ de toda t-álgebra de posto $3, \mathrm{~A}=\mathrm{Fe} \oplus \mathrm{U} \oplus \mathrm{V}$. É claro que os subespaços que compõem o Grupo I deveriam estar no Grupo II, mas não vamos reescrevê-los neste grupo. Resta aqui uma questão 
importante: saber se um subespaço do Grupo II deve permanecer aí ou pode ir para o Grupo I. Na seção 5.4, vamos tratar um pouco desta questão.

1. U

2. $\mathrm{V}$

3. $\mathrm{U}^{2}$

4. UV

5. $\mathrm{U}^{3}$

6. $\mathrm{U}(\mathrm{UV})$

7. (UV)V

8. $\mathrm{U}^{4}$

9. $\mathrm{U}[\mathrm{U}(\mathrm{UV})]$

10. $\mathrm{U}[(\mathrm{UV}) \mathrm{V}]$

11. $\mathrm{U}^{3} \mathrm{~V}$

12. $[(\mathrm{UV}) \mathrm{V}] \mathrm{V}$

14. $\mathrm{U} \oplus \mathrm{U}(\mathrm{UV})$

15. $\mathrm{U} \oplus \mathrm{U}^{4}$

16. $\mathrm{U} \oplus \mathrm{U}[(\mathrm{UV}) \mathrm{V}]$

18. $\mathrm{U}^{3} \oplus \mathrm{V}$

19. $(\mathrm{UV}) \mathrm{V} \oplus \mathrm{V}$

20. $\mathrm{U}[\mathrm{U}(\mathrm{UV})] \ominus \mathrm{V}$

21. $\mathrm{U}^{3} \mathrm{~V} \oplus \mathrm{V}$

22. $[(\mathrm{UV}) \mathrm{V}] \mathrm{V} \oplus \mathrm{V}$

25. (UV) $\mathrm{V} \oplus \mathrm{U}^{2}$

26. $\mathrm{U}[\mathrm{U}(\mathrm{UV})] \oplus \mathrm{U}^{2}$

27. $\mathrm{U}^{3} \mathrm{~V} \oplus \mathrm{U}^{2}$

28. $[(\mathrm{UV}) \mathrm{V}] \mathrm{V} \oplus \mathrm{U}^{2}$

30. $\mathrm{UV} \oplus \mathrm{U}^{4}$

31. $\mathrm{UV} \oplus \mathrm{U}[(\mathrm{UV}) \mathrm{V}]$

33. $U^{3}+(U V) V$

35. $\mathrm{U}^{3} \oplus \mathrm{U}[(\mathrm{UV}) \mathrm{V}]$

36. $\mathrm{U}^{3}+[(\mathrm{UV}) \mathrm{V}] \mathrm{V}$ 
37. $(\mathrm{UV}) \mathrm{V} \oplus \mathrm{U}(\mathrm{UV})$

39. $\mathrm{U}^{3} \mathrm{~V} \oplus \mathrm{U}(\mathrm{UV})$

40. $[(\mathrm{UV}) \mathrm{V}] \mathrm{V} \oplus \mathrm{U}(\mathrm{UV})$

41. $(\mathrm{UV}) \mathrm{V} \oplus \mathrm{U}^{4}$

42. $(\mathrm{UV}) \mathrm{V}+\mathrm{U}[\mathrm{U}(\mathrm{UV})]$

45. $\mathrm{U}^{4}+\mathrm{U}[(\mathrm{UV}) \mathrm{V}]$

46. $\mathrm{U}^{3} \mathrm{~V} \oplus \mathrm{U}^{4}$

47. $[(\mathrm{UV}) \mathrm{V}] \mathrm{V} \oplus \mathrm{U}^{4}$

48. $\mathrm{U}[\mathrm{U}(\mathrm{UV})] \oplus \mathrm{U}[(\mathrm{UV}) \mathrm{V}]$

49. $\mathrm{U}[\mathrm{U}(\mathrm{UV})]+[(\mathrm{UV}) \mathrm{V}] \mathrm{V}$

51. $[(\mathrm{UV}) \mathrm{V}] \mathrm{V} \oplus \mathrm{U}[(\mathrm{UV}) \mathrm{V}]$

52. $\mathrm{U}^{3} \mathrm{~V}+[(\mathrm{UV}) \mathrm{V}] \mathrm{V}$

53. $\mathrm{U} \oplus\left\{\mathrm{U}^{4}+\mathrm{U}[(\mathrm{UV}) \mathrm{V}]\right\}$

54. $\left[\mathrm{U}^{3}+(\mathrm{UV}) \mathrm{V}\right] \oplus \mathrm{V}$

55. $\left\{\mathrm{U}^{3}+[(\mathrm{UV}) \mathrm{V}] \mathrm{V}\right\} \oplus \mathrm{V}$

56. $\{(\mathrm{UV}) \mathrm{V}+\mathrm{U}[\mathrm{U}(\mathrm{UV})]\} \oplus \mathrm{V}$

57. $\{\mathrm{U}[\mathrm{U}(\mathrm{UV})]+[(\mathrm{UV}) \mathrm{V}] \mathrm{V}\} \oplus \mathrm{V}$

58. $\left\{\mathrm{U}^{3} \mathrm{~V}+[(\mathrm{UV}) \mathrm{V}] \mathrm{V}\right\} \oplus \mathrm{V}$

61. $\{(\mathrm{UV}) \mathrm{V}+\mathrm{U}[\mathrm{U}(\mathrm{UV})]\} \oplus \mathrm{U}^{2}$

62. $\{\mathrm{U}[\mathrm{U}(\mathrm{UV})]+[(\mathrm{UV}) \mathrm{V}] \mathrm{V}\} \oplus \mathrm{U}^{2}$

63. $\left\{\mathrm{U}^{3} \mathrm{~V}+[(\mathrm{UV}) \mathrm{V}] \mathrm{V}\right\} \oplus \mathrm{U}^{2}$

64. $\mathrm{UV} \oplus\left\{\mathrm{U}^{4}+\mathrm{U}[(\mathrm{UV}) \mathrm{V}]\right\}$

67. $\left[\mathrm{U}^{3}+(\mathrm{UV}) \mathrm{V}\right] \oplus \mathrm{U}^{4}$

68. $\left[\mathrm{U}^{3}+(\mathrm{UV}) \mathrm{V}\right] \oplus \mathrm{U}[(\mathrm{UV}) \mathrm{V}]$

70. $\left\{\mathrm{U}^{3}+[(\mathrm{UV}) \mathrm{V}] \mathrm{V}\right\} \oplus \mathrm{U}^{4}$

71. $\left\{\mathrm{U}^{3}+[(\mathrm{UV}) \mathrm{V}] \mathrm{V}\right\} \oplus \mathrm{U}[(\mathrm{UV}) \mathrm{V}]$

74. $\left\{\mathrm{U}^{3} \mathrm{~V}+[(\mathrm{UV}) \mathrm{V}] \mathrm{V}\right\} \oplus \mathrm{U}(\mathrm{UV})$

75. $\{(\mathrm{UV}) \mathrm{V}+\mathrm{U}[\mathrm{U}(\mathrm{UV})]\} \oplus \mathrm{U}^{4}$

76. $(\mathrm{UV}) \mathrm{V} \oplus\left\{\mathrm{U}^{4}+\mathrm{U}[(\mathrm{UV}) \mathrm{V}]\right\}$

77. $\{(\mathrm{UV}) \mathrm{V}+\mathrm{U}[\mathrm{U}(\mathrm{UV})]\} \oplus \mathrm{U}[(\mathrm{UV}) \mathrm{V}]$

79. $\{\mathrm{U}[\mathrm{U}(\mathrm{UV})]+[(\mathrm{UV}) \mathrm{V}] \mathrm{V}\} \oplus \mathrm{U}^{4}$

80. $\quad \mathrm{U}^{3} \mathrm{~V} \oplus\left\{\mathrm{U}^{4}+\mathrm{U}[(\mathrm{UV}) \mathrm{V}]\right\}$

81. $[(\mathrm{UV}) \mathrm{V}] \mathrm{V} \oplus\left\{\mathrm{U}^{4}+\mathrm{U}[(\mathrm{UV}) \mathrm{V}]\right\}$ 
82. $\left\{\mathrm{U}^{3} \mathrm{~V}+[(\mathrm{UV}) \mathrm{V}] \mathrm{V}\right\} \oplus \mathrm{U}^{4}$

83. $\{\mathrm{U}[\mathrm{U}(\mathrm{UV})]+[(\mathrm{UV}) \mathrm{V}] \mathrm{V}\} \oplus \mathrm{U}[(\mathrm{UV}) \mathrm{V}]$

89. $\left\{\mathrm{U}^{3} \mathrm{~V}+[(\mathrm{UV}) \mathrm{V}] \mathrm{V}\right\} \oplus\left\{\mathrm{U}^{4}+\mathrm{U}[(\mathrm{UV}) \mathrm{V}]\right\}$

\subsection{Sobre o Grupo II}

Vamos dedicar algum tempo à questão colocada no final da seção anterior. Para mostrar que um P-subespaço que compõe o Grupo II efetivamente não pertence ao Grupo I, precisamos encontrar um exemplo de uma t-álgebra de posto 3 mostrando que os subespaços $\mathrm{p}(\mathrm{U}, \mathrm{V})$ e $\mathrm{p}\left(\mathrm{U}_{0}, \mathrm{~V}_{0}\right)$, embora tenham mesma dimensão, são distintos para algum idempotente $\mathrm{e}_{0}$. Para alguns casos, já conseguimos encontrar este exemplo, como veremos a seguir.

Exemplo 16: Consideremos a família $\left\{\mathrm{A}_{r}\right\}$ de t-álgebras de posto 3 na qual, para cada $\mathrm{A}_{r}$, tem-se $\operatorname{dim} \mathrm{A}_{r}=2 r+1$ sobre o corpo $\mathrm{F}$, com base $\left\{\mathrm{e}, \mathrm{u}_{1}, \mathrm{u}_{2}, \ldots \mathrm{u}_{r}, \mathrm{u}_{1}^{2}, \mathrm{u}_{2}^{2}, \ldots \mathrm{u}_{r}^{2}\right\}$ e tábua de multiplicação dada por

$$
\left\{\begin{array}{l}
\mathrm{e}^{2}=\mathrm{e} ; 2 \mathrm{eu}_{\mathrm{i}}=\mathrm{u}_{\mathrm{i}}, \quad \mathrm{eu}_{\mathrm{i}}^{2}=\mathrm{u}_{\mathrm{i}}^{2},(\mathrm{i}=1, \ldots, \mathrm{r}) \\
\text { os outros produtos são nulos }
\end{array}\right.
$$

A função peso $\omega$ de $\mathrm{A}_{r}$ vale $1 \mathrm{em} e$ e 0 nos demais elementos da base e $\mathrm{A}_{r}$ satisfaz a t-equação $\mathrm{x}^{3}-2 \omega(\mathrm{x}) \mathrm{x}^{2}+\omega(\mathrm{x})^{2} \mathrm{x}=0$ e nesse caso, $\gamma=1$. Não vamos repetir aqui a prova de que $\mathrm{A}_{r}$ satisfaz esta equação pois é um simples cálculo. Portanto o conjunto dos elementos idempotentes de peso 1 de $A_{r}$ é dado por $\mathrm{I}\left(\mathrm{A}_{r}\right)=\left\{\mathrm{e}+\mathrm{u}-\mathrm{u}^{2}: \mathrm{u} \in \mathrm{U}\right\}$. Considerando o idempotente $\mathrm{e}_{0}=\mathrm{e}+\mathrm{u}_{2}-\mathrm{u}_{2}^{2}$ de $\mathrm{A}_{r}$, temos que $\mathrm{U}_{0}=$ $=\left\{\mathrm{u}-2 \mathrm{u}_{2} \mathrm{u}: \mathrm{u} \in \mathrm{U}\right\}, \mathrm{V}_{0}=\mathrm{V}$ e, dessa forma, o elemento $\mathrm{u}_{2}-2 \mathrm{u}_{2}^{2}$ é um elemento de $U_{0}$ que não pertence a $U$, o que mostra que $U$ não é invariante por mudança de idempotentes. Portanto $U$ efetivamente não pertence ao Grupo I. Além disso, temos que $\mathrm{U}^{2}=\mathrm{Fu}_{1}^{2} \oplus \mathrm{Fu}_{2}^{2} \oplus \ldots \oplus$ $\oplus \mathrm{Fu}_{r}^{2}$ e os subespaços monomiais UV. $\mathrm{U}^{3}, \mathrm{U}(\mathrm{UV})$. (UV)V. $\mathrm{U}^{4}, \mathrm{U}[\mathrm{U}(\mathrm{UV})]$. 
U[(UV)V], $\mathrm{U}^{3} \mathrm{~V},[(\mathrm{UV}) \mathrm{V}] \mathrm{V}$ são todos nulos. Dessa forma, obtemos, neste exemplo, a seguinte simplificação para alguns dos subespaços do Grupo II:

1. $\mathrm{U}$

14. $\mathrm{U} \oplus \mathrm{U}(\mathrm{UV})=\mathrm{U}$

15. $\mathrm{U} \oplus \mathrm{U}^{4}=\mathrm{U}$

16. $\mathrm{U} \oplus \mathrm{U}[(\mathrm{UV}) \mathrm{V}]=\mathrm{U}$

53. $\mathrm{U} \oplus\left\{\mathrm{U}^{4}+\mathrm{U}[(\mathrm{UV}) \mathrm{V}]\right\}=\mathrm{U}$

o que mostra que estes 5 subespaços de números $1,14,15,16,53$ efetivamente devem permanecer no Grupo II.

Exemplo 17: Consideremos a t-álgebra A de posto 3 sobre o corpo $\mathrm{F}$, com base $\left\{e, u_{1}, u_{2}, v_{1}, v_{2}\right\}$ e tábua de multiplicação dada por

$$
\left\{\begin{array}{l}
\mathrm{e}^{2}=\mathrm{e} ; 2 \mathrm{eu}_{1}=\mathrm{u}_{1} ; \quad \mathrm{eu}_{2}=\mathrm{u}_{2} \\
\mathrm{ev}_{1}=\mathrm{v}_{1} ; \quad \mathrm{ev}_{2}=\mathrm{v}_{2} ; \quad \mathrm{u}_{1} \mathrm{v}_{2}=\mathrm{u}_{2} \\
\text { os outros produtos são nulos }
\end{array}\right.
$$

e função peso definida por $\omega(\mathrm{e})=1$ e $\omega$ se anula nos demais vetores. Então A satisfaz $x^{3}-2 \omega(x) x^{2}+\omega(x)^{2} x=0$ e nesse caso, $\gamma=1$. Como $\mathrm{U}^{2}=0$, o conjunto dos elementos idempotentes de peso 1 de A é dado por $\mathrm{I}(\mathrm{A})=\{\mathrm{e}+\mathrm{u}: \mathrm{u} \in \mathrm{U}\}$, que é uma família a 2 parâmetros de idempotentes. Considerando o idempotente $\mathrm{e}_{0}=\mathrm{e}+\mathrm{u}_{1}$ de $\mathrm{A}$, temos que $\mathrm{U}_{0}=\mathrm{U}$ e $\mathrm{V}_{0}=\left\{2 \mathrm{bu}_{2}+\mathrm{av}_{1}+\mathrm{bv}_{2}: \mathrm{a}, \mathrm{b} \in \mathrm{F}\right\}$ e, dessa forma, o elemento $2 \mathrm{u}_{2}+\mathrm{v}_{1}+\mathrm{v}_{2}$ é um elemento de $\mathrm{V}_{0}$ que não pertence a $\mathrm{V}$, o que mostra que $\mathrm{V}$ não é invariante por mudança de idempotentes e, portanto, não pertence ao Grupo I. Além disso, temos que UV $=\mathrm{Fu}_{2}$ e os subespaços monomiais $\mathrm{U}^{2}, \mathrm{U}^{3}, \mathrm{U}(\mathrm{UV}),(\mathrm{UV}) \mathrm{V}, \mathrm{U}^{4}, \mathrm{U}[\mathrm{U}(\mathrm{UV})], \mathrm{U}[(\mathrm{UV}) \mathrm{V}], \mathrm{U}^{3} \mathrm{~V},[(\mathrm{UV}) \mathrm{V}] \mathrm{V}$ são todos nulos. Portanto obtemos a seguinte simplificação, neste exemplo, para alguns subespaços do Grupo II: 
2. $\mathrm{V}$

18. $\mathrm{U}^{3} \oplus \mathrm{V}=\mathrm{V}$

19. $(\mathrm{UV}) \mathrm{V} \oplus \mathrm{V}=\mathrm{V}$

20. $\mathrm{U}[\mathrm{U}(\mathrm{UV})] \oplus \mathrm{V}=\mathrm{V}$

21. $\mathrm{U}^{3} \mathrm{~V} \oplus \mathrm{V}=\mathrm{V}$

22. $[(\mathrm{UV}) \mathrm{V}] \mathrm{V} \oplus \mathrm{V}=\mathrm{V}$

54. $\left[\mathrm{U}^{3}+(\mathrm{UV}) \mathrm{V}\right] \oplus \mathrm{V}=\mathrm{V}$

55. $\left\{\mathrm{U}^{3}+[(\mathrm{UV}) \mathrm{V}] \mathrm{V}\right\} \oplus \mathrm{V}=\mathrm{V}$

56. $\{(\mathrm{UV}) \mathrm{V}+\mathrm{U}[\mathrm{U}(\mathrm{UV})]\} \oplus \mathrm{V}=\mathrm{V}$

57. $\{\mathrm{U}[\mathrm{U}(\mathrm{UV})]+[(\mathrm{UV}) \mathrm{V}] \mathrm{V}\} \oplus \mathrm{V}=\mathrm{V}$

58. $\left\{\mathrm{U}^{3} \mathrm{~V}+[(\mathrm{UV}) \mathrm{V}] \mathrm{V}\right\} \oplus \mathrm{V}=\mathrm{V}$

o que mostra que também estes subespaços de números $2,18,19,20,21$, $22,54,55,56,57,58$ devem efetivamente pertencer ao Grupo II.

Exemplo 18: Consideremos a t-álgebra A de posto 3 e dimensão 8 sobre o corpo $\mathrm{F}$, com base $\left\{\mathrm{e}, \mathrm{u}_{1}, \mathrm{u}_{2}, \mathrm{u}_{3}, \mathrm{u}_{4}, \mathrm{v}_{1}, \mathrm{v}_{2}, \mathrm{v}_{3}\right\}$ e tábua de multiplicação dada por

$$
\left\{\begin{array}{l}
\mathrm{e}^{2}=\mathrm{e} ; 2 \mathrm{eu}_{\mathrm{i}}=\mathrm{u}_{\mathrm{i}}(\mathrm{i}=1, \ldots, 4) ; \quad \mathrm{v}_{\mathrm{i}}=\mathrm{v}_{\mathrm{i}}(\mathrm{i}=1,2,3) \\
\mathrm{u}_{1} \mathrm{v}_{1}=\mathrm{u}_{4} ; \quad \mathrm{u}_{2} \mathrm{v}_{2}=\mathrm{u}_{4} ; \quad \mathrm{u}_{3} \mathrm{v}_{3}=-2 \mathrm{u}_{4} \\
2 \mathrm{u}_{1} \mathrm{u}_{2}=\mathrm{v}_{3} ; 2 \mathrm{u}_{1} \mathrm{u}_{3}=\mathrm{v}_{2} ; 2 \mathrm{u}_{2} \mathrm{u}_{3}=\mathrm{v}_{1} \\
\text { os outros produtos são nulos }
\end{array}\right.
$$

A função peso é definida na base de A por $\omega(\mathrm{e})=1$ e $\omega$ se anula nos demais elementos. Nesse caso $\gamma=1$, a álgebra $A$ satisfaz a t-equação $\mathrm{x}^{3}-2 \omega(\mathrm{x}) \mathrm{x}^{2}+\omega(\mathrm{x})^{2} \mathrm{x}=0$ e é fácil ver que $\mathrm{U}^{2}=\mathrm{V}$. O conjunto dos elementos idempotentes de peso 1 de $A$ é dado por $\mathrm{I}(\mathrm{A})=\left\{\mathrm{e}_{0}=\mathrm{e}+\mathrm{u}_{0}+\right.$ $\left.-\mathrm{u}_{0}^{2}: \mathrm{u}_{0} \in \mathrm{U}\right\}$. Considerando $\mathrm{e}_{0}=\mathrm{e}+\mathrm{u}_{1}$ um elemento de $\mathrm{I}(\mathrm{A})$, obtemos $\mathrm{U}_{0}=\mathrm{Fu}_{1} \oplus \mathrm{F}\left(\mathrm{u}_{2}-\mathrm{v}_{3}\right) \oplus \mathrm{F}\left(\mathrm{u}_{3}-\mathrm{v}_{2}\right) \oplus \mathrm{Fu}_{4}$ e portanto $2\left(\mathrm{u}_{2}-\mathrm{v}_{3}\right)\left(\mathrm{u}_{3}+\right.$ $\left.-\mathrm{v}_{2}\right)=2 \mathrm{u}_{2} \mathrm{u}_{3}-2 \mathrm{u}_{2} \mathrm{v}_{2}+2 \mathrm{u}_{3} \mathrm{v}_{3}+2 \mathrm{v}_{2} \mathrm{v}_{3}=\mathrm{v}_{1}+2 \mathrm{u}_{4}$ é um elemento de $\mathrm{U}_{0}^{2}$ que não pertence a $U^{2}$. o que mostra que $U^{2}$ não é invariante por mudançá 
de idempotentes e, dessa forma, não pertence ao Grupo I. Além disso. temos que $\mathrm{UV}=\mathrm{U}^{3}=\mathrm{Fu}_{4}$ e os subespaços monomiais $\mathrm{U}(\mathrm{UV}),(\mathrm{UV}) \mathrm{V}$, $\mathrm{U}^{4}, \mathrm{U}[\mathrm{U}(\mathrm{UV})], \mathrm{U}[(\mathrm{UV}) \mathrm{V}], \mathrm{U}^{3} \mathrm{~V},[(\mathrm{UV}) \mathrm{V}] \mathrm{V}$ são todos nulos. Portanto, com este exemplo, obtemos a seguinte simplificação para alguns subespaços do Grupo II:
3. $\mathrm{U}^{2}$
25. $(\mathrm{UV}) \mathrm{V} \oplus \mathrm{U}^{2}=\mathrm{U}^{2}$
26. $\mathrm{U}[(\mathrm{UV}) \mathrm{V}] \oplus \mathrm{U}^{2}=\mathrm{U}^{2}$
27. $\mathrm{U}^{3} \mathrm{~V} \oplus \mathrm{U}^{2}=\mathrm{U}^{2}$
28. $[(\mathrm{UV}) \mathrm{V}] \mathrm{V} \oplus \mathrm{U}^{2}=\mathrm{U}^{2}$
61. $\{(\mathrm{UV}) \mathrm{V}+\mathrm{U}[\mathrm{U}(\mathrm{UV})]\} \oplus \mathrm{U}^{2}=\mathrm{U}^{2}$
62. $\{\mathrm{U}[\mathrm{U}(\mathrm{UV})]+[(\mathrm{UV}) \mathrm{V}] \mathrm{V}\} \oplus \mathrm{U}^{2}=\mathrm{U}^{2}$
63. $\left\{\mathrm{U}^{3} \mathrm{~V}+[(\mathrm{UV}) \mathrm{V}] \mathrm{V}\right\} \oplus \mathrm{U}^{2}=\mathrm{U}^{2}$

e isto mostra que também estes subespaços de números 3, 25, 26, 27, 28, 61, 62, 63 devem efetivamente pertencer ao Grupo II.

Exemplo 19: Consideremos a t-álgebra A de posto 3 e dimensão 8 sobre o corpo $\mathrm{F}$, com base $\left\{\mathrm{e}, \mathrm{u}_{1}, \mathrm{u}_{2}, \mathrm{u}_{3}, \mathrm{u}_{4}, \mathrm{v}_{1}, \mathrm{v}_{2}, \mathrm{v}_{3}\right\}$ e tábua de multiplicação dada por

$$
\left\{\begin{array}{l}
\mathrm{e}^{2}=\mathrm{e} ; 2 \mathrm{eu}_{\mathrm{i}}=\mathrm{u}_{\mathrm{i}}(\mathrm{i}=1, \ldots, 4) ; \quad \mathrm{ev}_{\mathrm{i}}=\mathrm{v}_{\mathrm{i}}(\mathrm{i}=1,2,3) \\
2 \mathrm{u}_{1} \mathrm{v}_{1}=\mathrm{u}_{4} ; 2 \mathrm{u}_{2} \mathrm{v}_{1}=\mathrm{u}_{3} \\
2 \mathrm{u}_{1} \mathrm{u}_{2}=\mathrm{v}_{2} ; \mathrm{u}_{1} \mathrm{u}_{3}=\mathrm{v}_{3} ; \mathrm{u}_{2} \mathrm{u}_{4}=-\mathrm{v}_{3} \\
\text { os outros produtos são nulos }
\end{array}\right.
$$

Definimos a função peso na base de $\mathrm{A}$ por $\omega(\mathrm{e})=1$ e $\omega$ se anula nos demais vetores. Também nesse caso $\gamma=1$, a álgebra A satisfaz a tequação $x^{3}-2 \omega(x) x^{2}+\omega(x)^{2} x=0$ e o conjunto dos elementos idempotentes de peso 1 de $A$ é dado por $\mathrm{I}(\mathrm{A})=\left\{\mathrm{e}_{0}=\mathrm{e}+\mathrm{u}_{0}-\mathrm{u}_{0}^{2}: \mathrm{u}_{0} \in\right.$ $\in \mathrm{U}\}$. Considerando o idempotente $\mathrm{e}_{0}=\mathrm{e}+\mathrm{u}_{1}$ de $\mathrm{A}$, teremos $\mathrm{U}_{0}=\mathrm{Fu}_{1}$ (1) 
$\oplus \mathrm{F}\left(\mathrm{u}_{2}-\mathrm{v}_{2}\right) \oplus \mathrm{F}\left(\mathrm{u}_{3}-2 \mathrm{v}_{3}\right) \oplus \mathrm{Fu}_{4} \quad$ e $\mathrm{V}_{0}=\mathrm{F}\left(\mathrm{v}_{1}+\mathrm{u}_{4}\right) \oplus \mathrm{Fv}_{2} \oplus \mathrm{Fv}_{3}$. Portanto $2\left(\mathrm{u}_{2}-\mathrm{v}_{2}\right)\left(\mathrm{v}_{1}+\mathrm{u}_{4}\right)=2 \mathrm{u}_{2} \mathrm{v}_{1}+2 \mathrm{u}_{2} \mathrm{u}_{4}=\mathrm{u}_{3}-2 \mathrm{v}_{3}$ é um elemento de $\mathrm{U}_{0} \mathrm{~V}_{0}$ que não pertence a $\mathrm{UV}$, uma vez que $\mathrm{UV}=\mathrm{Fu}_{3} \oplus \mathrm{Fu}_{4}$. Com isso, fica mostrado que UV não é invariante por mudança de idempotentes e portanto não pertence ao Grupo I. Além disso, temos que $\mathrm{U}^{2}=\mathrm{Fv}_{2} \oplus$ (1) $\mathrm{Fv}_{3}, \mathrm{U}(\mathrm{UV})=\mathrm{Fv}_{3}$ e os subespaços monomiais $\mathrm{U}^{3},(\mathrm{UV}) \mathrm{V}, \mathrm{U}^{4}$, $\mathrm{U}[\mathrm{U}(\mathrm{UV})], \mathrm{U}[(\mathrm{UV}) \mathrm{V}], \mathrm{U}^{3} \mathrm{~V},[(\mathrm{UV}) \mathrm{V}] \mathrm{V}$ são todos nulos, o que acarreta seguinte simplificação para alguns subespaços do Grupo II:
4. UV
30. $\mathrm{UV} \oplus \mathrm{U}^{4}=\mathrm{UV}$
31. $\mathrm{UV} \oplus \mathrm{U}[(\mathrm{UV}) \mathrm{V}]=\mathrm{UV}$
64. $\mathrm{UV} \oplus\left\{\mathrm{U}^{4}+\mathrm{U}[(\mathrm{UV}) \mathrm{V}]\right\}=\mathrm{UV}$

Dessa forma, fica mostrado que também estes subespaços de números 4 , 30, 31, 64 devem efetivamente pertencer ao Grupo II.

Exemplo 20: Consideremos a t-álgebra A de posto 3 sobre o corpo $\mathrm{F}$, com base $\left\{\mathrm{e}, \mathrm{u}_{1}, \mathrm{u}_{2}, \mathrm{u}_{3}, \mathrm{u}_{4}, \mathrm{u}_{5}, \mathrm{u}_{6}, \mathrm{u}_{7}, \mathrm{v}_{\mathrm{i}}, \mathrm{v}_{2}, \mathrm{v}_{3}, \mathrm{v}_{4}\right\}$ e tábua de multiplicação dada por

$$
\left\{\begin{array}{l}
\mathrm{e}^{2}=\mathrm{e} ; 2 \mathrm{eu}_{\mathrm{i}}=\mathrm{u}_{\mathrm{i}}(\mathrm{i}=1, \ldots, 7) ; \quad \mathrm{ev}_{\mathrm{i}}=0(\mathrm{i}=1, \ldots, 4) \\
-\mathrm{u}_{1} \mathrm{u}_{5}=\mathrm{u}_{2} \mathrm{u}_{4}=\mathrm{v}_{2} ;-\mathrm{u}_{1} \mathrm{u}_{6}=\mathrm{u}_{3} \mathrm{u}_{4}=\mathrm{v}_{3} ; \quad \mathrm{u}_{2} \mathrm{u}_{6}=-\mathrm{u}_{3} \mathrm{u}_{5}=\mathrm{v}_{1} \\
\mathrm{u}_{1} \mathrm{v}_{1}=\mathrm{u}_{2} \mathrm{v}_{3}=-\mathrm{u}_{3} \mathrm{v}_{2}=\mathrm{u}_{7} ; \quad \mathrm{u}_{1} \mathrm{v}_{4}=\mathrm{u}_{4} ; \quad \mathrm{u}_{2} \mathrm{v}_{4}=\mathrm{u}_{5} ; \quad \mathrm{u}_{3} \mathrm{v}_{4}=\mathrm{u}_{6} \\
\text { os outros produtos são nulos }
\end{array}\right.
$$

Como nos exemplos anteriores, a função peso é definida na base de $\mathrm{A}$ por $\omega(\mathrm{e})=1 \mathrm{e} \omega$ se anula nos outros vetores. Nesse caso $\gamma=0$ (e portanto $\lambda=1)$, A satisfaz a equação $\mathrm{x}^{3}-\omega(\mathrm{x}) \mathrm{x}^{2}=0$ e o conjunto dos elementos idempotentes de $\mathrm{A}$ é dado por $\mathrm{I}(\mathrm{A})=\left\{\mathrm{e}_{0}=\mathrm{e}+\mathrm{u}_{0}+\mathrm{u}_{0}^{2}: \mathrm{u}_{0} \in \mathrm{U}\right\}$. Considerando o idempotente $\mathrm{e}_{0}=\mathrm{e}+\mathrm{u}_{3}$, pela Proposição 2 um gerador do $\mathrm{P}$-subespaço $\mathrm{U}_{0}\left(\mathrm{U}_{0} \mathrm{~V}_{0}\right)$ é $\mathrm{y}=-2 \mathrm{u}_{3}\left[\mathrm{u}_{2}\left(\mathrm{u}_{1} \mathrm{v}_{4}\right)\right] \ominus \mathrm{u}_{2}\left(\mathrm{u}_{1} \mathrm{v}_{4}\right)=-2 \mathrm{u}_{3}\left(\mathrm{u}_{2} \mathrm{u}_{4}\right) \ominus$ 
$\oplus \mathrm{u}_{2} \mathrm{u}_{4}=-2 \mathrm{u}_{3} \mathrm{v}_{2} \oplus \mathrm{v}_{2}=2 \mathrm{u}_{7} \oplus \mathrm{v}_{2}$, o que mostra que $\mathrm{y} \in \mathrm{U}_{0}\left(\mathrm{U}_{0} \mathrm{~V}_{0}\right)$, porém $\mathrm{y} \notin \mathrm{U}(\mathrm{UV})$. Ou seja, U(UV) não é invariante. Da tábua de multiplicação, é fácil ver que $\mathrm{U}^{2}=\mathrm{U}(\mathrm{UV})=\mathrm{Fv}_{1} \oplus \mathrm{Fv}_{2} \oplus \mathrm{Fv}_{3}, \mathrm{UV}=\mathrm{Fu}_{4} \oplus \mathrm{Fu}_{5} \oplus \mathrm{Fu}_{6} \oplus$ $\oplus \mathrm{Fu}_{7}, \mathrm{U}^{3}=\mathrm{U}[\mathrm{U}(\mathrm{UV})]=\mathrm{Fu}_{7}$ e os P-subespaços (UV)V, $\mathrm{U}^{4}, \mathrm{U}[(\mathrm{UV}) \mathrm{V}]$, $\mathrm{U}^{3} \mathrm{~V}$, [(UV)V]V são todos nulos. Obtemos, dessa forma, a seguinte simplificação para alguns subespaços do Grupo II:
6. $\mathrm{U}(\mathrm{UV})$
37. $(\mathrm{UV}) \mathrm{V} \oplus \mathrm{U}(\mathrm{UV})=\mathrm{U}(\mathrm{UV})$
39. $\mathrm{U}^{3} \mathrm{~V} \oplus \mathrm{U}(\mathrm{UV})=\mathrm{U}(\mathrm{UV})$
40. $[(\mathrm{UV}) \mathrm{V}] \mathrm{V} \oplus \mathrm{U}(\mathrm{UV})=\mathrm{U}(\mathrm{UV})$
74. $\left\{\mathrm{U}^{3} \mathrm{~V}+[(\mathrm{UV}) \mathrm{V}] \mathrm{V}\right\} \oplus \mathrm{U}(\mathrm{UV})=\mathrm{U}(\mathrm{UV})$

o que mostra que estes 5 subespaços de números $6,37,39,40$ e 74 efetivamente devem permanecer no Grupo II.

Exemplo 21: Consideremos a t-álgebra A de posto 3 sobre o corpo $\mathrm{F}$, com base $\left\{\mathrm{e}, \mathrm{u}_{1}, \mathrm{u}_{2}, \mathrm{u}_{3}, \mathrm{u}_{4}, \mathrm{u}_{5}, \mathrm{u}_{6}, \mathrm{u}_{7}, \mathrm{u}_{8}, \mathrm{v}_{1}, \mathrm{v}_{2}, \mathrm{v}_{3}\right\}$ e tábua de multiplicação

$$
\left\{\begin{array}{l}
\mathrm{e}^{2}=\mathrm{e} ; 2 \mathrm{eu}_{\mathrm{i}}=\mathrm{u}_{\mathrm{i}}(\mathrm{i}=1, \ldots 8) ; \quad \text { ev } \mathrm{i}=0 \quad(\mathrm{i}=1,2,3) \\
\mathrm{u}_{1} \mathrm{u}_{4}=\mathrm{u}_{1} \mathrm{u}_{5}=\mathrm{u}_{3} \mathrm{u}_{4}=\mathrm{v}_{3} \\
\mathrm{u}_{1} \mathrm{u}_{7}=\mathrm{u}_{2} \mathrm{u}_{3}=\mathrm{u}_{2} \mathrm{u}_{6}=\mathrm{u}_{2} \mathrm{u}_{8}=\mathrm{u}_{5} \mathrm{u}_{6}=-\mathrm{v}_{3} \\
\mathrm{u}_{1} \mathrm{v}_{1}=\mathrm{u}_{3} ; \quad \mathrm{u}_{1} \mathrm{v}_{2}=\mathrm{u}_{6} ; \quad \mathrm{u}_{2} \mathrm{v}_{1}=\mathrm{u}_{5} ; \quad \mathrm{u}_{2} \mathrm{v}_{2}=\mathrm{u}_{4} \\
\mathrm{u}_{4} \mathrm{v}_{1}=-\mathrm{u}_{5} \mathrm{v}_{2}=\mathrm{u}_{7} ; \quad \mathrm{u}_{3} \mathrm{v}_{2}=-\mathrm{u}_{6} \mathrm{v}_{1}=\mathrm{u}_{8} \\
\text { os outros produtos são nulos }
\end{array}\right.
$$

Novamente aqui definimos a função peso na base de A por $\omega(\mathrm{e})=1$ e $\omega$ é nula para os outros vetores. Dessa forma, $\gamma=0$. A satisfaz a t-equação $\mathrm{x}^{3}-\omega(\mathrm{x}) \mathrm{x}^{2}=0$ e o conjunto dos elementos idempotentes de $\mathrm{A}$ é dado por $\mathrm{I}(\mathrm{A})=\left\{\mathrm{e}_{0}=\mathrm{e}+\mathrm{u}_{0}+\mathrm{u}_{0}^{2}: \mathrm{u}_{0} \in \mathrm{U}\right\}$. Considerando o idempotente $\mathrm{e}_{0}=\mathrm{e}+\mathrm{u}_{2}$, pela Proposição $3 \mathrm{um}$ gerador do $\mathrm{P}$-subespaço $\left(\mathrm{U}_{0} \mathrm{~V}_{0}\right) \mathrm{V}_{0}$ é 
$\mathrm{y}=\left(\mathrm{u}_{1} \mathrm{v}_{2}\right) \mathrm{v}_{1} \oplus 2 \mathrm{u}_{2}\left[\left(\mathrm{u}_{1} \mathrm{v}_{2}\right) \mathrm{v}_{1}\right]=\mathrm{u}_{6} \mathrm{v}_{1} \oplus 2 \mathrm{u}_{2}\left(\mathrm{u}_{6} \mathrm{v}_{1}\right)=-\mathrm{u}_{8} \oplus 2 \mathrm{v}_{3}$. Ou seja, $\mathrm{y} \in\left(\mathrm{U}_{0} \mathrm{~V}_{0}\right) \mathrm{V}_{0}$ mas y $\notin(\mathrm{UV}) \mathrm{V}$, o que mostra que (UV)V não é invariante por mudança de idempotentes. Além disso, é fácil ver que $\mathrm{UV}=\mathrm{Fu}_{3} \oplus$ $\oplus \ldots \oplus \mathrm{Fu}_{8},(\mathrm{UV}) \mathrm{V}=\mathrm{Fu}_{7} \oplus \mathrm{Fu}_{8}, \mathrm{U}^{2}=\mathrm{U}(\mathrm{UV})=\mathrm{U}[(\mathrm{UV}) \mathrm{V}]=\mathrm{Fv}_{3}$ e os P-subespaços $U^{3}, U^{4}, U[U(U V)], U^{3} V$ e [(UV)V]V são todos nulos. Dessa forma, temos a seguinte simplificação para alguns subespaços do Grupo II:

$$
\begin{aligned}
\text { 7. } & (\mathrm{UV}) \mathrm{V} \\
\text { 33. } & \mathrm{U}^{3}+(\mathrm{UV}) \mathrm{V}=(\mathrm{UV}) \mathrm{V} \\
\text { 41. } & (\mathrm{UV}) \mathrm{V} \oplus \mathrm{U}^{4}=(\mathrm{UV}) \mathrm{V} \\
\text { 42. } & (\mathrm{UV}) \mathrm{V}+\mathrm{U}[\mathrm{U}(\mathrm{UV})]=(\mathrm{UV}) \mathrm{V} \\
\text { 67. } & {\left[\mathrm{U}^{3}+(\mathrm{UV}) \mathrm{V}\right] \oplus \mathrm{U}^{4}=(\mathrm{UV}) \mathrm{V} } \\
\text { 75. } & \{(\mathrm{UV}) \mathrm{V}+\mathrm{U}[\mathrm{U}(\mathrm{UV})]\} \oplus \mathrm{U}^{4}=(\mathrm{UV}) \mathrm{V}
\end{aligned}
$$

o que mostra que mais estes 6 subespaços de números $7,33,41,42,67$ e 75 efetivamente devem permanecer no Grupo II.

Exemplo 22: Consideremos agora a t-álgebra A de posto 3 sobre o corpo $\mathrm{F}$, com base $\left\{\mathrm{e}, \mathrm{u}_{1}, \mathrm{u}_{2}, \mathrm{u}_{3}, \mathrm{u}_{4}, \mathrm{u}_{5}, \mathrm{u}_{6}, \mathrm{u}_{\overline{7}}, \mathrm{u}_{8}, \mathrm{u}_{9}, \mathrm{u}_{10}, \mathrm{u}_{11}, \mathrm{u}_{12}, \mathrm{v}_{1}, \mathrm{v}_{2}, \mathrm{v}_{3}\right.$, $\left.\mathrm{v}_{4}, \mathrm{v}_{5}, \mathrm{v}_{6}, \mathrm{v}_{7}\right\}$ e tábua de multiplicação

$$
\left\{\begin{array}{l}
\mathrm{e}^{2}=\mathrm{e} ; 2 \mathrm{eu}_{\mathrm{i}}=\mathrm{u}_{\mathrm{i}}(\mathrm{i}=1, \ldots, 12) ; \quad \mathrm{v}_{\mathrm{i}}=0 \quad(\mathrm{i}=1, \ldots 7) \\
\mathrm{u}_{2}^{2}=\mathrm{v}_{3} ; \quad \mathrm{u}_{1} \mathrm{u}_{2}=\mathrm{v}_{4} ;-\mathrm{u}_{1} \mathrm{u}_{5}=\mathrm{u}_{2} \mathrm{u}_{3}=\mathrm{v}_{5} \\
\mathrm{u}_{1} \mathrm{u}_{4}=-\mathrm{u}_{2} \mathrm{u}_{6}=\mathrm{v}_{6} ; \quad \mathrm{u}_{1} \mathrm{u}_{7}=\mathrm{u}_{2} \mathrm{u}_{8}=-\mathrm{u}_{3} \mathrm{u}_{4}=\mathrm{u}_{5} \mathrm{u}_{6}=-\mathrm{v}_{7} \\
\mathrm{u}_{1} \mathrm{v}_{1}=\mathrm{u}_{3} ; \quad \mathrm{u}_{1} \mathrm{v}_{2}=\mathrm{u}_{6} ; \quad \mathrm{u}_{2} \mathrm{v}_{1}=\mathrm{u}_{5} ; \quad \mathrm{u}_{2} \mathrm{v}_{2}=\mathrm{u}_{4} ; \quad \mathrm{u}_{4} \mathrm{v}_{1}=-\mathrm{u}_{5} \mathrm{v}_{2}=\mathrm{u}_{7} \\
\mathrm{u}_{3} \mathrm{v}_{2}=-\mathrm{u}_{6} \mathrm{v}_{1}=\mathrm{u}_{8} ; \mathrm{u}_{1} \mathrm{v}_{3}=-2 \mathrm{u}_{2} \mathrm{v}_{4}=\mathrm{u}_{9} \\
-2 \mathrm{u}_{2} \mathrm{v}_{5}=\mathrm{u}_{3} \mathrm{v}_{3}=-2 \mathrm{u}_{5} \mathrm{v}_{4}=-\mathrm{u}_{9} \mathrm{v}_{1}=\mathrm{u}_{10} \\
2 \mathrm{u}_{2} \mathrm{v}_{6}=-2 \mathrm{u}_{4} \mathrm{v}_{4}=\mathrm{u}_{6} \mathrm{v}_{3}=-\mathrm{u}_{9} \mathrm{v}_{2}=\mathrm{u}_{11} \\
2 \mathrm{u}_{2} \mathrm{v}_{7}=-2 \mathrm{u}_{4} \mathrm{v}_{5}=-2 \mathrm{u}_{5} \mathrm{v}_{6}=2 \mathrm{u}_{7} \mathrm{v}_{4}=\mathrm{u}_{8} \mathrm{v}_{3}=-\mathrm{u}_{10} \mathrm{v}_{2}=\mathrm{u}_{11} \mathrm{v}_{1}=\mathrm{u}_{12} \\
\text { os outros produtos são nulos }
\end{array}\right.
$$

A função peso é definida na base de A como nos casos anteriores: $\omega(\mathrm{e})=$ 
$=1$ e $\omega$ se anula nos outros vetores. Como $\gamma=0$, segue que a t-equação satisfeita por A é $\mathrm{x}^{3}-\omega(\mathrm{x}) \mathrm{x}^{2}=0$ e o conjunto dos elementos idempotentes de $A$ é dado por $\mathrm{I}(\mathrm{A})=\left\{\mathrm{e}_{0}=\mathrm{e}+\mathrm{u}_{0}+\mathrm{u}_{0}^{2}: \mathrm{u}_{0} \in \mathrm{U}\right\}$. Considerando $\mathrm{u}_{0}=$ $=\mathrm{u}_{2}$, pela Proposição $9 \mathrm{um}$ gerador do P-subespaço $\mathrm{U}_{0}\left[\left(\mathrm{U}_{0} \mathrm{~V}_{0}\right) \mathrm{V}_{0}\right]$ é dado por $\mathrm{y}=-2 \mathrm{u}_{2}\left\{\mathrm{u}_{2}\left[\left(\mathrm{u}_{1} \mathrm{v}_{2}\right) \mathrm{v}_{1}\right]\right\} \oplus \mathrm{u}_{2}\left[\left(\mathrm{u}_{1} \mathrm{v}_{2}\right) \mathrm{v}_{1}\right]=-2 \mathrm{u}_{2}\left[\mathrm{u}_{2}\left(\mathrm{u}_{6} \mathrm{v}_{1}\right)\right] \oplus \mathrm{u}_{2}\left(\mathrm{u}_{6} \mathrm{v}_{1}\right)=$ $=-2 \mathrm{u}_{2}\left[\mathrm{u}_{2}\left(-\mathrm{u}_{8}\right)\right] \oplus \mathrm{u}_{2}\left(-\mathrm{u}_{8}\right)=2 \mathrm{u}_{2}\left(-\mathrm{v}_{7}\right) \oplus \mathrm{v}_{7}=-\mathrm{u}_{12} \oplus \mathrm{v}_{7}$. Portanto, $\mathrm{y} \in$ $\in \mathrm{U}_{0}\left[\left(\mathrm{U}_{0} \mathrm{~V}_{0}\right) \mathrm{V}_{0}\right]$ mas y $\notin \mathrm{U}[(\mathrm{UV}) \mathrm{V}]$, o que significa que o P-subespaço $\mathrm{U}[(\mathrm{UV}) \mathrm{V}]$ não é invariante por mudança de idempotentes. Além disso, temos que $\mathrm{U}^{2}=\mathrm{Fv}_{3} \oplus \ldots \oplus \mathrm{Fv}_{7}, \mathrm{UV}=\mathrm{Fu}_{3} \oplus \ldots \oplus \mathrm{Fu}_{12}, \mathrm{U}^{3}=$ $=\mathrm{Fu}_{9} \oplus \ldots \oplus \mathrm{Fu}_{12}, \mathrm{U}(\mathrm{UV})=\mathrm{Fv}_{5} \oplus \mathrm{Fv}_{6} \oplus \mathrm{Fv}_{7},(\mathrm{UV}) \mathrm{V}=\mathrm{Fu}_{7} \oplus \mathrm{Fu}_{8} \oplus$ $\oplus \mathrm{Fu}_{10} \oplus \mathrm{Fu}_{11} \oplus \mathrm{Fu}_{12}$ (isto é, $\mathrm{u}_{9} \notin(\mathrm{UV}) \mathrm{V}$ ), $\mathrm{U}[\mathrm{U}(\mathrm{UV})]=\mathrm{U}^{3} \mathrm{~V}=\mathrm{Fu}_{10} \oplus$ $\oplus \mathrm{Fu}_{11} \oplus \mathrm{Fu}_{12}, \mathrm{U}[(\mathrm{UV}) \mathrm{V}]=\mathrm{Fv}_{7},[(\mathrm{UV}) \mathrm{V}] \mathrm{V}=\mathrm{Fu}_{12}$ e $\mathrm{U}^{4}=0$. Temos, assim, a seguinte simplificação para alguns subespaços do Grupo II:

$$
\begin{array}{ll}
\text { 10. } & \mathrm{U}[(\mathrm{UV}) \mathrm{V}] \\
\text { 45. } & \mathrm{U}^{4}+\mathrm{U}[(\mathrm{UV}) \mathrm{V}]=\mathrm{U}[(\mathrm{UV}) \mathrm{V}]
\end{array}
$$

Isto mostra que os subespaços de números 10 e 45 efetivamente devem permanecer no Grupo II.

Exemplo 23: Consideremos agora a t-álgebra A de posto 3 sobre o corpo $\mathrm{F}$, com base $\left\{\mathrm{e}, \mathrm{u}_{1}, \mathrm{u}_{2}, \mathrm{u}_{3}, \mathrm{u}_{4}, \mathrm{u}_{5}, \mathrm{u}_{6}, \mathrm{v}_{1}, \mathrm{v}_{2}, \mathrm{v}_{3}, \mathrm{v}_{4}\right\}$ e tábua de multiplicação

$$
\left\{\begin{array}{l}
\mathrm{e}^{2}=\mathrm{e} ; 2 \mathrm{eu}_{\mathrm{i}}=\mathrm{u}_{\mathrm{i}}(\mathrm{i}=1, \ldots, 6) ; \quad \mathrm{vv}_{\mathrm{i}}=0(\mathrm{i}=1, \ldots, 4) \\
\mathrm{u}_{1}^{2}=\mathrm{v}_{1} ; \mathrm{u}_{1} \mathrm{u}_{2}=\mathrm{v}_{2} ; \mathrm{u}_{1} \mathrm{u}_{3}=\mathrm{v}_{3} ; 2 \mathrm{u}_{1} \mathrm{u}_{6}=-\mathrm{u}_{2} \mathrm{u}_{5}=\mathrm{u}_{3} \mathrm{u}_{4}=\mathrm{v}_{4} \\
-2 \mathrm{u}_{1} \mathrm{v}_{2}=\mathrm{u}_{2} \mathrm{v}_{1}=\mathrm{u}_{4} ;-2 \mathrm{u}_{1} \mathrm{v}_{3}=\mathrm{u}_{3} \mathrm{v}_{1}=\mathrm{u}_{5} ;-\mathrm{u}_{2} \mathrm{v}_{3}=\mathrm{u}_{3} \mathrm{v}_{2}=\mathrm{u}_{6} \\
\text { os outros produtos são nulos }
\end{array}\right.
$$

A função peso é definida na base de A por $\omega(\mathrm{e})=1$ e $\omega$ se anula nos outros vetores. Temos que $\gamma=0$ e portanto t-equação satisfeita por A é $\mathrm{x}^{3}-\omega(\mathrm{x}) \mathrm{x}^{2}=0$. O conjunto dos elementos idempotentes de A novamente 
é dado por $\mathrm{I}(\mathrm{A})=\left\{\mathrm{e}_{0}=\mathrm{e}+\mathrm{u}_{0}+\mathrm{u}_{0}^{2}: \mathrm{u}_{0} \in \mathrm{U}\right\}$. Se tomarmos $\mathrm{u}_{0}=$ $=\mathrm{u}_{3}$, então pela Proposição $5 \mathrm{um}$ gerador do P-subespaço $\mathrm{U}_{0}^{3}$ é $\mathrm{y}=$ $=\mathrm{u}_{1}^{2} \mathrm{u}_{2} \oplus 2 \mathrm{u}_{3}\left(\mathrm{u}_{1}^{2} \mathrm{u}_{2}\right)=\mathrm{u}_{2} \mathrm{v}_{1} \oplus 2 \mathrm{u}_{3}\left(\mathrm{u}_{2} \mathrm{v}_{1}\right)=\mathrm{u}_{4} \oplus 2 \mathrm{v}_{4}$. Assim, $\mathrm{y} \in \mathrm{U}_{0}^{3}$ mas y $\notin \mathrm{U}^{3}$, o que significa que o $\mathrm{P}$-subespaço $\mathrm{U}^{3}$ não é invariante. Além disso, temos que $\mathrm{U}^{2}=\mathrm{Fv}_{1} \oplus \mathrm{Fv}_{2} \oplus \mathrm{Fv}_{3} \oplus \mathrm{Fv}_{4}, \mathrm{UV}=\mathrm{U}^{3}=\mathrm{Fu}_{4} \oplus$ $\oplus \mathrm{Fu}_{5} \oplus \mathrm{Fu}_{6}, \mathrm{U}(\mathrm{UV})=\mathrm{U}^{4}=\mathrm{Fv}_{4}$ e os P-subespaços (UV)V, U[U(UV)], $\mathrm{U}[(\mathrm{UV}) \mathrm{V}], \mathrm{U}^{3} \mathrm{~V},[(\mathrm{UV}) \mathrm{V}] \mathrm{V}$ são todos nulos. Obtemos, dessa forma, a seguinte simplificação para alguns subespaços do Grupo II:
5. $\mathrm{U}^{3}$
35. $\mathrm{U}^{3} \oplus \mathrm{U}[(\mathrm{UV}) \mathrm{V}]=\mathrm{U}^{3}$
36. $\mathrm{U}^{3}+[(\mathrm{UV}) \mathrm{V}] \mathrm{V}=\mathrm{U}^{3}$
68. $\left[\mathrm{U}^{3}+(\mathrm{UV}) \mathrm{V}\right] \oplus \mathrm{U}[(\mathrm{UV}) \mathrm{V}]=\mathrm{U}^{3}$
71. $\left\{\mathrm{U}^{3}+[(\mathrm{UV}) \mathrm{V}] \mathrm{V}\right\} \oplus \mathrm{U}[(\mathrm{UV}) \mathrm{V}]=\mathrm{U}^{3}$

Com isto, fica mostrado que os subespaços de números 5, 35, 36, 68 e 71 efetivamente devem permanecer no Grupo II.

Exemplo 24: Consideremos a t-álgebra A de posto 3 sobre o corpo $\mathrm{F}$, com base $\left\{e, u_{1}, \ldots . u_{11}, v_{1}, \ldots, v_{8}\right\}$ e tábua de multiplicação dada por

$$
\left\{\begin{array}{l}
\mathrm{e}^{2}=\mathrm{e} ; 2 \mathrm{eu}_{\mathrm{i}}=\mathrm{u}_{\mathrm{i}}(\mathrm{i}=1, \ldots, 11) ; \quad \mathrm{v}_{\mathrm{i}}=0(\mathrm{i}=1, \ldots, 8) \\
\mathrm{u}_{1}^{2}=\mathrm{v}_{1} ; \quad \mathrm{u}_{3}^{2}=-\mathrm{v}_{1}+2 \mathrm{v}_{3} ; \mathrm{u}_{1} \mathrm{u}_{2}=\mathrm{u}_{2} \mathrm{u}_{3}=\mathrm{v}_{2} ; \quad \mathrm{u}_{1} \mathrm{u}_{3}=\mathrm{v}_{3} \\
2 \mathrm{u}_{2} \mathrm{u}_{10}=-\mathrm{v}_{6}+2 \mathrm{v}_{8} ; \quad \mathrm{u}_{1} \mathrm{u}_{4}=\mathrm{u}_{3} \mathrm{u}_{4}=\mathrm{v}_{4}:-\mathrm{u}_{3} \mathrm{u}_{9}=\mathrm{u}_{4} \mathrm{u}_{8}=\mathrm{v}_{8} \\
2 \mathrm{u}_{1} \mathrm{u}_{10}=-\mathrm{u}_{3} \mathrm{u}_{7}=-2 \mathrm{u}_{3} \mathrm{u}_{10}=\mathrm{u}_{4} \mathrm{u}_{6}=\mathrm{v}_{7} \\
2 \mathrm{u}_{1} \mathrm{u}_{8}=-\mathrm{u}_{2} \mathrm{u}_{6}=\mathrm{u}_{3} \mathrm{u}_{5}=\mathrm{v}_{5} ; 2 \mathrm{u}_{1} \mathrm{u}_{9}=-\mathrm{u}_{2} \mathrm{u}_{7}=\mathrm{u}_{4} \mathrm{u}_{5}=\mathrm{v}_{6} \\
-2 \mathrm{u}_{1} \mathrm{v}_{2}=\mathrm{u}_{2} \mathrm{v}_{1}=\mathrm{u}_{5} ;-2 \mathrm{u}_{1} \mathrm{v}_{4}=\mathrm{u}_{4} \mathrm{v}_{1}=\mathrm{u}_{7} \\
-2 \mathrm{u}_{1} \mathrm{v}_{3}=\mathrm{u}_{3} \mathrm{v}_{1}=2 \mathrm{u}_{3} \mathrm{v}_{3}=\mathrm{u}_{6} \\
\mathrm{u}_{3} \mathrm{v}_{2}=\mathrm{u}_{8}:-\mathrm{u}_{2} \mathrm{v}_{4}=\mathrm{u}_{4} \mathrm{v}_{2}=\mathrm{u}_{9}: \mathrm{u}_{4} \mathrm{v}_{3}=\mathrm{u}_{10} \\
-2 \mathrm{u}_{1} \mathrm{v}_{8}=\mathrm{u}_{2} \mathrm{v}_{7}=-\mathrm{u}_{3} \mathrm{v}_{6}=\mathrm{u}_{4} \mathrm{v}_{5}=\mathrm{u}_{11} \\
2 \mathrm{u}_{2} \mathrm{v}_{3}=\mathrm{u}_{5}-2 \mathrm{u}_{8} ; 2 \mathrm{u}_{3} \mathrm{v}_{4}=\mathrm{u}_{7}-2 \mathrm{u}_{10} \\
\text { os outros produtos são nulos }
\end{array}\right.
$$


Definimos a função peso na base de A por $\omega(\mathrm{e})=1$ e $\omega$ se anula nos outros vetores. Além disso, $\gamma=0$ e a t-equação satisfeita por $\mathrm{A}$ é $\mathrm{x}^{3}-\omega(\mathrm{x}) \mathrm{x}^{2}=$ $=0$. Novamente conjunto dos elementos idempotentes de A é dado por $\mathrm{I}(\mathrm{A})=\left\{\mathrm{e}_{0}=\mathrm{e}+\mathrm{u}_{0}+\mathrm{u}_{0}^{2}: \mathrm{u}_{0} \in \mathrm{U}\right\}$. Considerando agora o idempotente $\mathrm{e}_{0}=\mathrm{e}+\mathrm{u}_{4}$, pela Proposição $6 \mathrm{um}$ gerador do $\mathrm{P}$-subespaço $\mathrm{U}_{0}^{4}$ é $\mathrm{y}=$ $\left.=-2 \mathrm{u}_{4}\left[\mathrm{u}_{3}\left(\mathrm{u}_{2} \mathrm{u}_{1}^{2}\right)\right] \oplus \mathrm{u}_{3}\left(\mathrm{u}_{2} \mathrm{u}_{1}^{2}\right)\right)=-2 \mathrm{u}_{4}\left[\mathrm{u}_{3}\left(\mathrm{u}_{2} \mathrm{v}_{1}\right)\right] \oplus \mathrm{u}_{3}\left(\mathrm{u}_{2} \mathrm{v}_{1}\right)=-2 \mathrm{u}_{4}\left(\mathrm{u}_{3} \mathrm{u}_{5}\right) \oplus$ $\oplus \mathrm{u}_{3} \mathrm{u}_{5}=-2 \mathrm{u}_{4} \mathrm{v}_{5} \oplus \mathrm{v}_{5}=-2 \mathrm{u}_{11} \oplus \mathrm{v}_{5}$. Ou seja, $\mathrm{y} \in \mathrm{U}_{0}^{4}$ mas $\mathrm{y} \notin \mathrm{U}^{4}$, o que mostra que $\mathrm{U}^{4}$ não é um $\mathrm{P}$-subespaço invariante. Da tábua de multiplicação, vemos que $\mathrm{U}^{2}=\mathrm{V}, \mathrm{UV}=\mathrm{U}^{3}=\mathrm{Fu}_{5} \oplus \ldots \oplus \mathrm{Fu}_{11}$, $\mathrm{U}(\mathrm{UV})=\mathrm{U}^{4}=\mathrm{Fv}_{5} \oplus \ldots \oplus \mathrm{Fv}_{8}, \mathrm{U}^{5}=\mathrm{U}[\mathrm{U}(\mathrm{UV})]=\mathrm{Fu}_{11},(\mathrm{UV}) \mathrm{V}=$ $=\mathrm{U}[(\mathrm{UV}) \mathrm{V}]=[(\mathrm{UV}) \mathrm{V}] \mathrm{V}=\mathrm{U}^{3} \mathrm{~V}=0$. Com isto, obtemos a seguinte simplificação para alguns subespaços do Grupo II:
8. $\mathrm{U}^{4}$
46. $\quad \mathrm{U}^{3} \mathrm{~V} \oplus \mathrm{U}^{4}=\mathrm{U}^{4}$
47. $[(\mathrm{UV}) \mathrm{V}] \mathrm{V} \oplus \mathrm{U}^{4}=\mathrm{U}^{4}$
76. $(\mathrm{UV}) \mathrm{V} \oplus\left\{\mathrm{U}^{4}+\mathrm{U}[(\mathrm{UV}) \mathrm{V}]\right\}=\mathrm{U}^{4}$
80. $\mathrm{U}^{3} \mathrm{~V} \oplus\left\{\mathrm{U}^{4}+\mathrm{U}[(\mathrm{UV}) \mathrm{V}]\right\}=\mathrm{U}^{4}$
81. $[(\mathrm{UV}) \mathrm{V}] \mathrm{V} \oplus\left\{\mathrm{U}^{4}+\mathrm{U}[(\mathrm{UV}) \mathrm{V}]\right\}=\mathrm{U}^{4}$
82. $\left\{\mathrm{U}^{3} \mathrm{~V}+[(\mathrm{UV}) \mathrm{V}] \mathrm{V}\right\} \oplus \mathrm{U}^{4}=\mathrm{U}^{4}$
89. $\left\{\mathrm{U}^{3} \mathrm{~V}+[(\mathrm{UV}) \mathrm{V}] \mathrm{V}\right\} \oplus\left\{\mathrm{U}^{4}+\mathrm{U}[(\mathrm{UV}) \mathrm{V}]\right\}=\mathrm{U}^{4}$

e dessa forma temos que estes 8 subespaços de números $8,46,47,76,80$, 81, 82 e 89 efetivamente devem permanecer no Grupo II.

Exemplo 25: Consideremos a t-álgebra A de posto 3 sobre o corpo $\mathrm{F}$, com base $\left\{e, u_{1}, \ldots, u_{16}, v_{1}, \ldots, v_{10}\right\}$ e tábua de multiplicação dada por 


$$
\left\{\begin{array}{l}
\mathrm{e}^{2}=\mathrm{e} ; 2 \mathrm{eu}_{\mathrm{i}}=\mathrm{u}_{\mathrm{i}}(\mathrm{i}=1, \ldots, 16) ; \quad \text { ev }=0(\mathrm{i}=1, \ldots, 10) \\
\mathrm{u}_{1} \mathrm{u}_{6}=-\mathrm{u}_{2} \mathrm{u}_{3}=\mathrm{v}_{4} ; \mathrm{u}_{1} \mathrm{u}_{7}=-\mathrm{u}_{2} \mathrm{u}_{4}=\mathrm{v}_{5} ; \mathrm{u}_{1} \mathrm{u}_{8}=-\mathrm{u}_{2} \mathrm{u}_{5}=\mathrm{v}_{6} \\
\mathrm{u}_{1} \mathrm{u}_{12}=-\mathrm{u}_{2} \mathrm{u}_{9}=\mathrm{u}_{3} \mathrm{u}_{7}=-\mathrm{u}_{4} \mathrm{u}_{6}=\mathrm{v}_{7} \\
\mathrm{u}_{1} \mathrm{u}_{13}=-\mathrm{u}_{2} \mathrm{u}_{10}=\mathrm{u}_{3} \mathrm{u}_{8}=-\mathrm{u}_{5} \mathrm{u}_{6}=\mathrm{v}_{8} \\
\mathrm{u}_{1} \mathrm{u}_{14}=-\mathrm{u}_{2} \mathrm{u}_{11}=\mathrm{u}_{4} \mathrm{u}_{8}=-\mathrm{u}_{5} \mathrm{u}_{7}=-\mathrm{v}_{9} \\
\mathrm{u}_{1} \mathrm{u}_{16}=\mathrm{u}_{3} \mathrm{u}_{14}=\mathrm{u}_{5} \mathrm{u}_{12}=\mathrm{u}_{6} \mathrm{u}_{11}=\mathrm{u}_{8} \mathrm{u}_{9}=\mathrm{v}_{10} \\
\mathrm{u}_{2} \mathrm{u}_{15}=\mathrm{u}_{4} \mathrm{u}_{13}=\mathrm{u}_{7} \mathrm{u}_{10}=-\mathrm{v}_{10} \\
\mathrm{u}_{1} \mathrm{v}_{1}=\mathrm{u}_{3} ; \mathrm{u}_{1} \mathrm{v}_{2}=\mathrm{u}_{4} ; \mathrm{u}_{1} \mathrm{v}_{3}=\mathrm{u}_{5} ; \mathrm{u}_{2} \mathrm{v}_{1}=\mathrm{u}_{6} ; \mathrm{u}_{2} \mathrm{v}_{2}=\mathrm{u}_{7} ; \mathrm{u}_{2} \mathrm{v}_{3}=\mathrm{u}_{8} \\
\mathrm{u}_{3} \mathrm{v}_{2}=-\mathrm{u}_{4} \mathrm{v}_{1}=\mathrm{u}_{9} ; \mathrm{u}_{3} \mathrm{v}_{3}=-\mathrm{u}_{5} \mathrm{v}_{1}=\mathrm{u}_{10} ; \mathrm{u}_{4} \mathrm{v}_{3}=-\mathrm{u}_{5} \mathrm{v}_{2}=\mathrm{u}_{11} \\
\mathrm{u}_{6} \mathrm{v}_{2}=-\mathrm{u}_{7} \mathrm{v}_{1}=\mathrm{u}_{12} ; \mathrm{u}_{6} \mathrm{v}_{3}=-\mathrm{u}_{8} \mathrm{v}_{1}=\mathrm{u}_{13} ; \mathrm{u}_{7} \mathrm{v}_{3}=-\mathrm{u}_{8} \mathrm{v}_{2}=\mathrm{u}_{14} \\
\mathrm{u}_{9} \mathrm{v}_{3}=-\mathrm{u}_{10} \mathrm{v}_{2}=\mathrm{u}_{11} \mathrm{v}_{1}=\mathrm{u}_{15} ; \mathrm{u}_{12} \mathrm{v}_{3}=-\mathrm{u}_{13} \mathrm{v}_{2}=\mathrm{u}_{14} \mathrm{v}_{1}=-\mathrm{u}_{16} \\
\text { os outros produtos são nulos }
\end{array}\right.
$$

Assim como nos exemplos anteriores, definimos a função peso na base de A por $\omega(\mathrm{e})=1$ e $\omega$ se anula nos outros vetores, $\gamma=0$ e a t-equação satisfeita por A é $\mathrm{x}^{3}-\omega(\mathrm{x}) \mathrm{x}^{2}=0$. Novamente o conjunto dos elementos idempotentes de A é dado por $\mathrm{I}(\mathrm{A})=\left\{\mathrm{e}_{0}=\mathrm{e}+\mathrm{u}_{0}+\mathrm{u}_{0}^{2}: \mathrm{u}_{0} \in \mathrm{U}\right\}$. Considerando agora o idempotente $\mathrm{e}_{0}=\mathrm{e}+\mathrm{u}_{2}$, pela Proposição $10 \mathrm{um}$ gerador do subespaço $\left[\left(\mathrm{U}_{0} \mathrm{~V}_{0}\right) \mathrm{V}_{0}\right] \mathrm{v}_{0}$ é $\mathrm{y}=\left[\left(\mathrm{u}_{1} \mathrm{v}_{1}\right) \mathrm{v}_{2}\right] \mathrm{v}_{3} \oplus 2 \mathrm{u}_{2}\left\{\left[\left(\mathrm{u}_{1} \mathrm{v}_{1}\right) \mathrm{v}_{2}\right] \mathrm{v}_{3}\right\}$ $=\left(\mathrm{u}_{3} \mathrm{v}_{2}\right) \mathrm{v}_{3} \oplus 2 \mathrm{u}_{2}\left[\left(\mathrm{u}_{3} \mathrm{v}_{2}\right) \mathrm{v}_{3}\right]=\mathrm{u}_{9} \mathrm{v}_{3} \oplus \mathrm{u}_{2}\left(\mathrm{u}_{9} \mathrm{v}_{3}\right)=\mathrm{u}_{15} \oplus\left(-2 \mathrm{v}_{10}\right)$ e portanto $\mathrm{y} \in\left[\left(\mathrm{U}_{0} \mathrm{~V}_{0}\right) \mathrm{V}_{0}\right] \mathrm{V}_{0}$ mas y $\notin[(\mathrm{UV}) \mathrm{V}] \mathrm{V}$, o que mostra que [(UV)V]V não é invariante. Além disso, é fácil ver que $\mathrm{U}^{2}=\mathrm{U}(\mathrm{UV})=\mathrm{Fv}_{4} \ominus \ldots \ominus$ $\oplus \mathrm{Fv}_{10}, \mathrm{UV}=\mathrm{Fu}_{3} \ominus \ldots \ominus \mathrm{Fu}_{16},(\mathrm{UV}) \mathrm{V}=\mathrm{Fu}_{9} \ominus \ldots \ominus \mathrm{Fu}_{16}, \mathrm{U}[(\mathrm{UV}) \mathrm{V}]=$ $=\mathrm{Fv}_{7} \oplus \ldots \oplus \mathrm{Fv}_{10},[(\mathrm{UV}) \mathrm{V}] \mathrm{V}=\mathrm{Fu}_{15} \oplus \mathrm{F}_{16}, \mathrm{U}^{3}=\mathrm{U}^{4}=\mathrm{U}^{3} \mathrm{~V}=$ $=\mathrm{U}[\mathrm{U}(\mathrm{UV})]=0$. Isto acarreta a seguinte simplificação para alguns subespaços do Grupo II:

$$
\begin{array}{ll}
\text { 12. } & {[(\mathrm{UV}) \mathrm{V}] \mathrm{V}} \\
\text { 49. } & \mathrm{U}[\mathrm{U}(\mathrm{UV})]+[(\mathrm{UV}) \mathrm{V}] \mathrm{V}=[(\mathrm{UV}) \mathrm{V}] \mathrm{V} \\
\text { 52. } & \mathrm{U}^{3} \mathrm{~V}+[(\mathrm{UV}) \mathrm{V}] \mathrm{V}=[(\mathrm{UV}) \mathrm{V}] \mathrm{V} \\
\text { 70. } & \left\{\mathrm{U}^{3}+[(\mathrm{UV}) \mathrm{V}] \mathrm{V}\right\} \ominus \mathrm{U}^{-1}=[(\mathrm{UV}) \mathrm{V}] \mathrm{V} \\
\text { 79. } & \{\mathrm{U}[\mathrm{U}(\mathrm{UV})]+[(\mathrm{UV}) \mathrm{V}] \mathrm{V}\} \oplus \mathrm{U}^{4}=[(\mathrm{UV}) \mathrm{V}] \mathrm{V}
\end{array}
$$


e dessa forma temos que estes 5 subespaços de números $12,49,52.70$ e 79 efetivamente devem permanecer no Grupo II.

O próximo exemplo é encontrado em [10, pag. 99].

Exemplo 26: Consideremos a t-álgebra A de posto 3 sobre o corpo $\mathrm{F}$, com base $\left\{e, u_{1}, \ldots, u_{16}, v_{1}, \ldots, v_{9}\right\}$ e tábua de multiplicação dada por

$$
\left\{\begin{array}{l}
\mathrm{e}^{2}=\mathrm{e} ; 2 \mathrm{eu}_{\mathrm{i}}=\mathrm{u}_{\mathrm{i}}(\mathrm{i}=1, \ldots, 16) ; \quad \text { ev } \mathrm{i}=0(\mathrm{i}=1, \ldots, 9) \\
\mathrm{u}_{1}^{2}=\mathrm{v}_{3} ; \mathrm{u}_{2}^{2}=\mathrm{v}_{4} ; \quad \mathrm{u}_{1} \mathrm{u}_{2}=\mathrm{v}_{5} \\
\mathrm{u}_{1} \mathrm{u}_{5}=-\mathrm{u}_{2} \mathrm{u}_{3}=-\mathrm{v}_{6} ; \quad \mathrm{u}_{1} \mathrm{u}_{6}=-\mathrm{u}_{2} \mathrm{u}_{4}=\mathrm{v}_{7} \\
\mathrm{u}_{1} \mathrm{u}_{9}=\mathrm{u}_{2} \mathrm{u}_{10}=\mathrm{u}_{3} \mathrm{u}_{6}=-\mathrm{u}_{4} \mathrm{u}_{5}=-\mathrm{v}_{8} \\
\mathrm{u}_{1} \mathrm{u}_{16}=\mathrm{u}_{2} \mathrm{u}_{15}=\mathrm{u}_{3} \mathrm{u}_{14}=\mathrm{u}_{7} \mathrm{u}_{9}=\mathrm{v}_{9} \\
\mathrm{u}_{4} \mathrm{u}_{11}=\mathrm{u}_{5} \mathrm{u}_{12}=\mathrm{u}_{6} \mathrm{u}_{13}=\mathrm{u}_{8} \mathrm{u}_{10}=-\mathrm{v}_{9} \\
\mathrm{u}_{1} \mathrm{v}_{1}=\mathrm{u}_{3} ; \mathrm{u}_{1} \mathrm{v}_{2}=\mathrm{u}_{4} ; \quad \mathrm{u}_{2} \mathrm{v}_{1}=\mathrm{u}_{5} ; \quad \mathrm{u}_{2} \mathrm{v}_{2}=\mathrm{u}_{6} \\
2 \mathrm{u}_{1} \mathrm{v}_{5}=-\mathrm{u}_{2} \mathrm{v}_{3}=-\mathrm{u}_{7} ; \quad \mathrm{u}_{1} \mathrm{v}_{4}=2 \mathrm{u}_{2} \mathrm{v}_{5}=-\mathrm{u}_{8} \\
\mathrm{u}_{5} \mathrm{v}_{2}=-\mathrm{u}_{6} \mathrm{v}_{1}=\mathrm{u}_{9} ; \quad \mathrm{u}_{3} \mathrm{v}_{2}=-\mathrm{u}_{4} \mathrm{v}_{1}=-\mathrm{u}_{10} \\
2 \mathrm{u}_{2} \mathrm{v}_{6}=-\mathrm{u}_{3} \mathrm{v}_{4}=2 \mathrm{u}_{5} \mathrm{v}_{5}=\mathrm{u}_{8} \mathrm{v}_{1}=-\mathrm{u}_{11} \\
2 \mathrm{u}_{1} \mathrm{v}_{7}=2 \mathrm{u}_{4} \mathrm{v}_{5}=-\mathrm{u}_{6} \mathrm{v}_{3}=\mathrm{u}_{7} \mathrm{v}_{2}=\mathrm{u}_{12} \\
2 \mathrm{u}_{1} \mathrm{v}_{6}=-2 \mathrm{u}_{3} \mathrm{v}_{5}=\mathrm{u}_{5} \mathrm{v}_{3}=-\mathrm{u}_{7} \mathrm{v}_{1}=\mathrm{u}_{13} \\
2 \mathrm{u}_{2} \mathrm{v}_{7}=\mathrm{u}_{4} \mathrm{v}_{4}=-2 \mathrm{u}_{6} \mathrm{v}_{5}=-\mathrm{u}_{8} \mathrm{v}_{2}=\mathrm{u}_{14} \\
2 \mathrm{u}_{1} \mathrm{v}_{8}=2 \mathrm{u}_{3} \mathrm{v}_{7}=2 \mathrm{u}_{4} \mathrm{v}_{6}=\mathrm{u}_{9} \mathrm{v}_{3}=2 \mathrm{u}_{10} \mathrm{v}_{5}=-\mathrm{u}_{12} \mathrm{v}_{1}=-\mathrm{u}_{13} \mathrm{v}_{2}=-\mathrm{u}_{15} \\
2 \mathrm{u}_{2} \mathrm{v}_{8}=2 \mathrm{u}_{5} \mathrm{v}_{7}=2 \mathrm{u}_{6} \mathrm{v}_{6}=2 \mathrm{u}_{9} \mathrm{v}_{5}=\mathrm{u}_{10} \mathrm{v}_{4}=\mathrm{u}_{11} \mathrm{v}_{2}=-\mathrm{u}_{14} \mathrm{v}_{1}=\mathrm{u}_{16} \\
\text { os outros produtos são nulos }
\end{array}\right.
$$

Definimos a função peso na base de A por $\omega(\mathrm{e})=1$ e $\omega$ se anula nos demais vetores. Aqui também temos $\gamma=0$, a t-equação satisfeita por A é $\mathrm{x}^{3}-\omega(\mathrm{x}) \mathrm{x}^{2}=0$ e o conjunto dos elementos idempotentes de A é dado por $\mathrm{I}(\mathrm{A})=\left\{\mathrm{e}_{0}=\mathrm{e}+\mathrm{u}_{0}+\mathrm{u}_{0}^{2}: \mathrm{u}_{0} \in \mathrm{U}\right\}$. Considerando o idempotente $\mathrm{e}_{0}=$ $=\mathrm{e}+\mathrm{u}_{1}+\mathrm{v}_{3}$, da Proposição 7 , o elemento $\mathrm{y}=\left(\mathrm{u}_{3} \mathrm{u}_{2}^{2}\right) \mathrm{v}_{2} \oplus 2 \mathrm{u}_{1}\left[\left(\mathrm{u}_{3} \mathrm{u}_{2}^{2}\right) \mathrm{v}_{2}\right]=$ $=\left(\mathrm{u}_{3} \mathrm{v}_{4}\right) \mathrm{v}_{2} \oplus 2 \mathrm{u}_{1}\left[\left(\mathrm{u}_{3} \mathrm{v}_{4}\right) \mathrm{v}_{2}\right]=\mathrm{u}_{11} \mathrm{v}_{2} \oplus 2 \mathrm{u}_{1}\left(\mathrm{u}_{11} \mathrm{v}_{2}\right)=\mathrm{u}_{16} \oplus 2 \mathrm{u}_{1} \mathrm{u}_{16}=$ 
$=\mathrm{u}_{16} \oplus 2 \mathrm{v}_{9}$ é um gerador de $\mathrm{U}_{0}^{3} \mathrm{~V}_{0}$ e daí segue que $\mathrm{y} \in \mathrm{U}_{0}^{3} \mathrm{~V}_{0}$. embora $\mathrm{y} \notin \mathrm{U}^{3} \mathrm{~V}$, o que mostra que $\mathrm{U}^{3} \mathrm{~V}$ não é invariante. Além disso. pela Proposição 8, segue que o mesmo elemento y acima considerado é um gerador de $\mathrm{U}_{0}\left[\mathrm{U}_{0}\left(\mathrm{U}_{0} \mathrm{~V}_{0}\right)\right]$ (omitiremos aqui os cálculos) e dessa forma $\mathrm{y} \in \mathrm{U}_{0}\left[\mathrm{U}_{0}\left(\mathrm{U}_{0} \mathrm{~V}_{0}\right)\right]$ mas y $\notin \mathrm{U}[\mathrm{U}(\mathrm{UV})]$ e com isso mostramos que $\mathrm{U}[\mathrm{U}(\mathrm{UV})]$ não é invariante por mudança de idempotentes. Logo, os seguintes subespaços do Grupo II

\section{2. $\mathrm{U}[\mathrm{U}(\mathrm{UV})]$ \\ 49. $\mathrm{U}^{3} \mathrm{~V}$}

devem efetivamente permanecer no Grupo II.

Restam ainda pendentes quatro casos: 48, 51, 77 e 83. Para decidir se eles ficam mesmo no Grupo II devemos encontrar exemplos análogos aos de acima. 


\section{Capítulo 6}

\section{Ideais de tipo polinomial}

Neste capítulo vamos procurar determinar quais dos P-subespaços de grau $\leq 4$ são ideais da t-álgebra $\mathrm{A}$.

\subsection{O estudo do Grupo I}

Tomemos uma t-álgebra, de posto $3, \mathrm{~A}=\mathrm{Fe} \oplus \mathrm{U} \oplus \mathrm{V}$. Inicialmente observamos que se $\mathrm{m}(\mathrm{U}, \mathrm{V})$ é um subespaço monomial então $\mathrm{m}(\mathrm{U}, \mathrm{V}) \subseteq$ $\subseteq \mathrm{U}$ ou $\mathrm{m}(\mathrm{U}, \mathrm{V}) \subseteq \mathrm{V}$. Assim se $\mathrm{x} \in \mathrm{m}(\mathrm{U}, \mathrm{V})$ então ex é múltiplo de $\mathrm{x}$ (pois $2 \mathrm{ex}=\mathrm{x}$ ou ex $=\gamma \mathrm{x}$ ) e portanto ex $\in \mathrm{m}(\mathrm{U}, \mathrm{V})$; ou seja, em(U, V) $\subseteq$ $\subseteq \mathrm{m}(\mathrm{U}, \mathrm{V})$ e dessa forma para determinar se $\mathrm{m}(\mathrm{U}, \mathrm{V})$ é um ideal de $\mathrm{A}$ basta ver se $m(U, V)$ é um ideal de $N=U \oplus V$. Em outras palavras. devemos apenas mostrar que $\mathrm{U}[\mathrm{m}(\mathrm{U}, \mathrm{V})]$ e $\mathrm{V}[\mathrm{m}(\mathrm{U}, \mathrm{V})]$ estão ambos em $\mathrm{m}(\mathrm{U}, \mathrm{V})$.

Para facilitar a leitura do próximo teorema, reestabelecemos aqui relações de inclusão que já apareceram anteriormente, entre alguns subespaços monomiais. São elas:

$$
\begin{aligned}
& \mathrm{U}(\mathrm{UV})^{2} \subseteq \mathrm{U}^{3} \mathrm{~V} \subseteq \mathrm{U}[\mathrm{U}(\mathrm{UV})] \subseteq \mathrm{U}^{3} \subseteq \mathrm{UV} \\
& \mathrm{U}[(\mathrm{UV}) \mathrm{V}]=(\mathrm{UV})^{2} \subseteq \mathrm{U}(\mathrm{UV}) \subseteq \mathrm{U}^{2} \\
& {[(\mathrm{UV}) \mathrm{V}] \mathrm{V} \subseteq(\mathrm{UV}) \mathrm{V} \subseteq \mathrm{UV} \subseteq \mathrm{U}} \\
& \mathrm{U}^{5} \subseteq \mathrm{U}[\mathrm{U}(\mathrm{UV})]
\end{aligned}
$$


Teorema 1 Todos os P-subespaços pertencentes ao Grupo I são ideais de $A$.

Prova: Como já vimos, $\mathrm{eM} \subseteq \mathrm{M}$ para todo subespaço monomial $\mathrm{M}$ de A. Resta então, mostrarmos para cada subespaço polinomial $\mathrm{p}(\mathrm{U}, \mathrm{V})$ do Grupo I, que $(\mathrm{u}+\mathrm{v}) \mathrm{p}(\mathrm{U}, \mathrm{V}) \subseteq \mathrm{p}(\mathrm{U}, \mathrm{V})$, com $\mathrm{u} \in \mathrm{U}$ e $\mathrm{v} \in \mathrm{V}$. Cada caso será identificado por seu correspondente número listado no Grupo I. Omitiremos os casos já conhecidos como, por exemplo, $\mathrm{U}^{n} \oplus \mathrm{U}^{n+1}$, $\mathrm{UV} \oplus \mathrm{V}, \mathrm{N}^{k}$.

29. De (i), segue que $(u+v)[U V \oplus U(U V)]=\{u(U V)+u[U(U V)]\}+$ $+\{\mathrm{v}(\mathrm{UV})+\mathrm{v}[\mathrm{U}(\mathrm{UV})]\} \subseteq[\mathrm{U}(\mathrm{UV})+\mathrm{UV}]+\mathrm{UV} \subseteq \mathrm{UV} \oplus \mathrm{U}(\mathrm{UV})$ e isto mostra que $U V \oplus U(U V)$ é um ideal de $A$.

32. Usando-se novamente (i), obtemos $(u+v)\left[U^{3} \oplus U(U V)\right]=\left\{u U^{3}+\right.$ $+\mathrm{u}[\mathrm{U}(\mathrm{UV})]\}+\left\{\mathrm{vU}^{3}+\mathrm{v}[\mathrm{U}(\mathrm{UV})]\right\} \subseteq\left[\mathrm{U}(\mathrm{UV})+\mathrm{U}^{3}\right]+\mathrm{U}^{3} \mathrm{~V} \subseteq$ $\subseteq\left[\mathrm{U}(\mathrm{UV})+\mathrm{U}^{3}\right]+\mathrm{U}^{3} \subseteq \mathrm{U}^{3} \oplus \mathrm{U}(\mathrm{UV})\left(\right.$ pois $\left.\mathrm{v}[\mathrm{U}(\mathrm{UV})] \subseteq \mathrm{V}^{2}=0\right) \mathrm{e}$ portanto $\mathrm{U}^{3} \oplus \mathrm{U}(\mathrm{UV})$ é um ideal de $\mathrm{A}$.

38. De (i) temos $(\mathrm{u}+\mathrm{v})\{\mathrm{U}[\mathrm{U}(\mathrm{UV})] \oplus \mathrm{U}(\mathrm{UV})\}=\{\mathrm{u}\{\mathrm{U}[\mathrm{U}(\mathrm{UV})]\}+$ $+\mathrm{u}[\mathrm{U}(\mathrm{UV})]\}+\{\mathrm{v}\{\mathrm{U}[\mathrm{U}(\mathrm{UV})]\}+\mathrm{v}[\mathrm{U}(\mathrm{UV})]\} \subseteq\{\mathrm{U}(\mathrm{UV})+\mathrm{U}[\mathrm{U}(\mathrm{UV})]\}+$ $+\{\mathrm{U}[\mathrm{U}(\mathrm{UV})]\} \mathrm{V} \subseteq\{\mathrm{U}(\mathrm{UV})+\mathrm{U}[\mathrm{U}(\mathrm{UV})]\}+\mathrm{U}^{3} \mathrm{~V} \subseteq \mathrm{U}[\mathrm{U}(\mathrm{UV})] \oplus \mathrm{U}(\mathrm{UV})$ e portanto $U[U(U V)] \oplus U(U V)$ é um ideal de $A$.

43. Basta ver que $(\mathrm{UV}) \mathrm{V} \oplus \mathrm{U}[(\mathrm{UV}) \mathrm{V}]=(\mathrm{UV} \oplus \mathrm{V})^{2}$ e portanto, de $[6$, Proposição 5], segue o resultado.

44. Usando-se agora (iv) e (i), temos $(\mathrm{u}+\mathrm{v})\left\{\mathrm{U}[\mathrm{U}(\mathrm{UV})] \oplus \mathrm{U}^{4}\right\}=$ $=\mathrm{u}\left\{\mathrm{U}[\mathrm{U}(\mathrm{UV})]+\mathrm{uU^{4 }}+\mathrm{v}\{\mathrm{U}[\mathrm{U}(\mathrm{UV})]\}+\mathrm{vU}^{4} \subseteq \mathrm{U}^{4}+\mathrm{U}^{5}+\mathrm{U}^{3} \mathrm{~V} \subseteq\right.$ $\subseteq \mathrm{U}[\mathrm{U}(\mathrm{UV})] \oplus \mathrm{U}^{4}$ e isto mostra que $\mathrm{U}[\mathrm{U}(\mathrm{UV})] \oplus \mathrm{U}^{4}$ é um ideal de $\mathrm{A}$.

50. De $U^{3} \mathrm{~V}=\left(\mathrm{U}^{2} \mathrm{U}\right) \mathrm{V} \subseteq(\mathrm{UV}) \mathrm{V}$ e $(\mathrm{i})$, segue que $(\mathrm{u}+\mathrm{v})\left\{\mathrm{U}^{3} \mathrm{~V} \oplus\right.$ $\left.\oplus \mathrm{U}[(\mathrm{UV}) \mathrm{V}]\}=\mathrm{u}\left(\mathrm{U}^{3} \mathrm{~V}\right)+\mathrm{u}\{\mathrm{U}[\mathrm{UV}) \mathrm{V}]\right\}+\mathrm{v}\left(\mathrm{U}^{3} \mathrm{~V}\right)+\mathrm{v}\{\mathrm{U}[(\mathrm{UV}) \mathrm{V}]\} \subseteq$ $\subseteq\left\{\mathrm{U}[(\mathrm{UV}) \mathrm{V}]+\mathrm{U}(\mathrm{UV})^{2}\right\}+\mathrm{U}^{3} \mathrm{~V} \subseteq \mathrm{U}^{3} \mathrm{~V} \oplus \mathrm{U}[(\mathrm{UV}) \mathrm{V}]$, logo o subespaço $\mathrm{U}^{3} \mathrm{~V} \oplus \mathrm{U}[(\mathrm{UV}) \mathrm{V}]$ é um ideal de $\mathrm{A}$. 
59. Como já sabemos que $\mathrm{U}^{3} \oplus \mathrm{U}^{2}$ é ideal de $\mathrm{A}$. basta mostrarmos que $(\mathrm{u}+\mathrm{v})[(\mathrm{UV}) \mathrm{V}] \subseteq\left[\mathrm{U}^{3}+(\mathrm{UV}) \mathrm{V}\right] \oplus \mathrm{U}^{2}$. Mas de (ii) e (iii), segue que $(\mathrm{u}+\mathrm{v})[(\mathrm{UV}) \mathrm{V}]=\mathrm{u}[(\mathrm{UV}) \mathrm{V}]+\mathrm{v}[(\mathrm{UV}) \mathrm{V}] \subseteq \mathrm{U}^{2}+(\mathrm{UV}) \mathrm{V} \subseteq\left[\mathrm{U}^{3}+\right.$ $+(\mathrm{UV}) \mathrm{V}] \oplus \mathrm{U}^{2}$ e isto mostra que $\left[\mathrm{U}^{3}+(\mathrm{UV}) \mathrm{V}\right] \oplus \mathrm{U}^{2}$ é um ideal de A.

60. Novamente usando-se que $U^{3} \oplus \mathrm{U}^{2}$ é ideal de $\mathrm{A}$, basta mostrarmos que $(\mathrm{u}+\mathrm{v})\{[(\mathrm{UV}) \mathrm{V}] \mathrm{V}\} \subseteq\left\{\mathrm{U}^{3}+[(\mathrm{UV}) \mathrm{V}] \mathrm{V}\right\} \oplus \mathrm{U}^{2}$. Mas, usando (iii), segue que $(\mathrm{u}+\mathrm{v})\{[(\mathrm{UV}) \mathrm{V}] \mathrm{V}\}=\mathrm{u}\{[(\mathrm{UV}) \mathrm{V}] \mathrm{V}\}+\mathrm{v}\{[(\mathrm{UV}) \mathrm{V}] \mathrm{V}\} \subseteq \mathrm{U}^{2}+$ $+[(\mathrm{UV}) \mathrm{V}] \mathrm{V} \subseteq\left\{\mathrm{U}^{3}+[(\mathrm{UV}) \mathrm{V}] \mathrm{V}\right\} \oplus \mathrm{U}^{2}$ e portanto $\left\{\mathrm{U}^{3}+[(\mathrm{UV}) \mathrm{V}] \mathrm{V}\right\} \oplus$ $\oplus \mathrm{U}^{2}$ é um ideal de $\mathrm{A}$.

66. Como já vimos que $\mathrm{U}^{3} \oplus \mathrm{U}(\mathrm{UV})$ é um ideal de $\mathrm{A}$, resta apenas mostrarmos que $(\mathrm{u}+\mathrm{v})\{[(\mathrm{UV}) \mathrm{V}] \mathrm{V}\} \subseteq\left\{\mathrm{U}^{3}+[(\mathrm{UV}) \mathrm{V}] \mathrm{V}\right\} \oplus \mathrm{U}(\mathrm{UV})$. $\operatorname{Mas}(\mathrm{u}+\mathrm{v})\{[(\mathrm{UV}) \mathrm{V}] \mathrm{V}\}=\mathrm{u}\{[(\mathrm{UV}) \mathrm{V}] \mathrm{V}\}+\mathrm{v}\{[(\mathrm{UV}) \mathrm{V}] \mathrm{V}\} \subseteq \mathrm{U}(\mathrm{UV}) \oplus$ $\oplus[(\mathrm{UV}) \mathrm{V}] \mathrm{V} \subseteq\left\{\mathrm{U}^{3}+[(\mathrm{UV}) \mathrm{V}] \mathrm{V}\right\} \oplus \mathrm{U}(\mathrm{UV})$. Portanto $\left\{\mathrm{U}^{3}+\right.$ $+[(\mathrm{UV}) \mathrm{V}] \mathrm{V}\} \oplus \mathrm{U}(\mathrm{UV})$ é um ideal de $\mathrm{A}$.

69. Já sabemos que $\mathrm{U}^{3} \oplus \mathrm{U}^{4}$ é um ideal de $\mathrm{A}$ e portanto resta apenas mostrarmos que $(\mathrm{u}+\mathrm{v})\{\mathrm{U}[(\mathrm{UV}) \mathrm{V}]\} \subseteq \mathrm{U}^{3} \oplus\left\{\mathrm{U}^{4}+\mathrm{U}[(\mathrm{UV}) \mathrm{V}]\right\}$. Mas $(\mathrm{u}+\mathrm{v})\{\mathrm{U}[(\mathrm{UV}) \mathrm{V}]\}=\mathrm{u}\{\mathrm{U}[(\mathrm{UV}) \mathrm{V}]\}+\mathrm{v}\{\mathrm{U}[(\mathrm{UV}) \mathrm{V}]\}=\mathrm{u}\{\mathrm{U}[(\mathrm{UV}) \mathrm{V}]\} \subseteq$ $\subseteq \mathrm{U}^{3} \subseteq \mathrm{U}^{3} \oplus\left\{\mathrm{U}^{4}+\mathrm{U}[(\mathrm{UV}) \mathrm{V}]\right\}$ o que completa a prova de que $\mathrm{U}^{3} \oplus$ $\oplus\left\{\mathrm{U}^{4}+\mathrm{U}[(\mathrm{UV}) \mathrm{V}]\right\}$ é um ideal de $\mathrm{A}$.

72. Como já sabemos que $\mathrm{U}[\mathrm{U}(\mathrm{UV})] \oplus \mathrm{U}(\mathrm{UV})$ é um ideal de $A$, resta apenas mostrarmos que $(\mathrm{u}+\mathrm{v})[(\mathrm{UV}) \mathrm{V}] \subseteq\{(\mathrm{UV}) \mathrm{V}+\mathrm{U}[\mathrm{U}(\mathrm{UV})]\} \oplus$ $\oplus \mathrm{U}(\mathrm{UV})$. Mas, de (iii), temos $(\mathrm{u}+\mathrm{v})[(\mathrm{UV}) \mathrm{V}]=\mathrm{u}[(\mathrm{UV}) \mathrm{V}]+\mathrm{v}[(\mathrm{UV}) \mathrm{V}] \subseteq$ $\subseteq \mathrm{U}(\mathrm{UV})+(\mathrm{UV}) \mathrm{V} \subseteq\{(\mathrm{UV}) \mathrm{V}+\mathrm{U}[\mathrm{U}(\mathrm{UV})]\} \oplus \mathrm{U}(\mathrm{UV})$ e dessa forma $\{(U V) V+U[U(U V)]\} \oplus U(U V)$ é um ideal de $A$.

73. Como já vimos que $\mathrm{U}[\mathrm{U}(\mathrm{UV})] \oplus \mathrm{U}(\mathrm{UV})$ é um ideal de $A$, basta mostrarmos que $(\mathrm{u}+\mathrm{v})\{[(\mathrm{UV}) \mathrm{V}] \mathrm{V}\} \subseteq\{\mathrm{U}[\mathrm{U}(\mathrm{UV})]+[(\mathrm{UV}) \mathrm{V}] \mathrm{V}\} \Theta$ $\oplus \mathrm{U}(\mathrm{UV})$. Mas de (iii), segue que $(\mathrm{u}+\mathrm{v})\{[(\mathrm{UV}) \mathrm{V}] \mathrm{V}\} \subseteq \mathrm{u}\{[(\mathrm{UV}) \mathrm{V}] \mathrm{V}\}+$ $+\mathrm{v}\{[(\mathrm{UV}) \mathrm{V}] \mathrm{V}\} \subseteq \mathrm{U}(\mathrm{UV})+[(\mathrm{UV}) \mathrm{V}] \mathrm{V} \subseteq\{\mathrm{U}[\mathrm{U}(\mathrm{UV})]+[(\mathrm{UV}) \mathrm{V}] \mathrm{V}\} \oplus$ 
$\oplus \mathrm{U}(\mathrm{UV})$ e dessa forma segue que $\{\mathrm{U}[\mathrm{U}(\mathrm{UV})]+[(\mathrm{UV}) \mathrm{V}] \mathrm{V}\} \oplus \mathrm{U}(\mathrm{UV})$ também é um ideal de A.

78. Já sabemos que $\mathrm{U}[\mathrm{U}(\mathrm{UV})] \oplus \mathrm{U}^{4}$ é um ideal de A e portanto resta $\operatorname{mostrarmos}$ que $(\mathrm{u}+\mathrm{v})\{\mathrm{U}[(\mathrm{UV}) \mathrm{V}]\} \subseteq \mathrm{U}[\mathrm{U}(\mathrm{UV})] \oplus\left\{\mathrm{U}^{4}+\mathrm{U}[(\mathrm{UV}) \mathrm{V}]\right\}$. Como temos $(\mathrm{u}+\mathrm{v})\{\mathrm{U}[(\mathrm{UV}) \mathrm{V}]\}=\mathrm{u}\{\mathrm{U}[(\mathrm{UV}) \mathrm{V}]\}+\mathrm{v}\{\mathrm{U}[(\mathrm{UV}) \mathrm{V}]\}=$ $=\mathrm{u}\{\mathrm{U}[(\mathrm{UV}) \mathrm{V}]\} \subseteq \mathrm{U}[\mathrm{U}(\mathrm{UV})] \subseteq \mathrm{U}[\mathrm{U}(\mathrm{UV})] \oplus\left\{\mathrm{U}^{4}+\mathrm{U}[(\mathrm{UV}) \mathrm{V}]\right\}$ segue que $\mathrm{U}[\mathrm{U}(\mathrm{UV})] \oplus\left\{\mathrm{U}^{4}+\mathrm{U}[(\mathrm{UV}) \mathrm{V}]\right\}$ é um ideal de $\mathrm{A}$.

84. Como já vimos que $\mathrm{U}^{3} \mathrm{~V} \oplus \mathrm{U}[(\mathrm{UV}) \mathrm{V}]$ é um ideal de $\mathrm{A}$, resta apenas mostrarmos que $(\mathrm{u}+\mathrm{v})\{[(\mathrm{UV}) \mathrm{V}] \mathrm{V}\} \subseteq\left\{\mathrm{U}^{3} \mathrm{~V}+[(\mathrm{UV}) \mathrm{V}] \mathrm{V}\right\} \oplus$ $\oplus \mathrm{U}[(\mathrm{UV}) \mathrm{V}]$. Mas de (iii), temos $(\mathrm{u}+\mathrm{v})\{[(\mathrm{UV}) \mathrm{V}] \mathrm{V}\}=\mathrm{u}\{[(\mathrm{UV}) \mathrm{V}] \mathrm{V}\}+$ $+\mathrm{v}\{[(\mathrm{UV}) \mathrm{V}] \mathrm{V}\} \subseteq \mathrm{U}[(\mathrm{UV}) \mathrm{V}]+[(\mathrm{UV}) \mathrm{V}] \mathrm{V} \subseteq\left\{\mathrm{U}^{3} \mathrm{~V}+[(\mathrm{UV}) \mathrm{V}] \mathrm{V}\right\} \oplus$ $\oplus \mathrm{U}[(\mathrm{UV}) \mathrm{V}]$ e daí concluimos que $\left\{\mathrm{U}^{3} \mathrm{~V}+[(\mathrm{UV}) \mathrm{V}] \mathrm{V}\right\} \oplus \mathrm{U}[(\mathrm{UV}) \mathrm{V}]$ é um ideal de A.

85. Como $U^{3} \oplus U^{4}$ e $(U V) V \oplus U[(U V) V]$ são ideais de $A$, segue que o subespaço $\left[\mathrm{U}^{3}+(\mathrm{UV}) \mathrm{V}\right] \oplus\left\{\mathrm{U}^{4}+\mathrm{U}[(\mathrm{UV}) \mathrm{V}]\right\}$ é um ideal de $\mathrm{A}$, como soma de dois ideais.

86. Já vimos que $\mathrm{U}^{3} \oplus\left\{\mathrm{U}^{4}+\mathrm{U}[(\mathrm{UV}) \mathrm{V}]\right\}$ é um ideal de $\mathrm{A}$ e portanto resta apenas mostrarmos que $(\mathrm{u}+\mathrm{v})\{[(\mathrm{UV}) \mathrm{V}] \mathrm{V}\} \subseteq\left\{\mathrm{U}^{3}+[(\mathrm{UV}) \mathrm{V}] \mathrm{V}\right\} \oplus$ $\oplus\left\{\mathrm{U}^{4}+\mathrm{U}[(\mathrm{UV}) \mathrm{V}]\right\}$. Mas, de (iii), segue que $(\mathrm{u}+\mathrm{v})\{[(\mathrm{UV}) \mathrm{V}] \mathrm{V}\}=$ $=\mathrm{u}\{[(\mathrm{UV}) \mathrm{V}] \mathrm{V}\}+\mathrm{v}\{[(\mathrm{UV}) \mathrm{V}] \mathrm{V}\} \subseteq \mathrm{U}[(\mathrm{UV}) \mathrm{V}]+[(\mathrm{UV}) \mathrm{V}] \mathrm{V} \subseteq \mathrm{U}^{3} \oplus$ $\oplus\left\{\mathrm{U}^{4}+\mathrm{U}[(\mathrm{UV}) \mathrm{V}]\right\}$ e isto mostra que $\left\{\mathrm{U}^{3}+[(\mathrm{UV}) \mathrm{V}] \mathrm{V}\right\} \oplus\left\{\mathrm{U}^{4}+\right.$ $+\mathrm{U}[(\mathrm{UV}) \mathrm{V}]\}$ é um ideal de A.

87. Como $(U V) V \oplus U[(U V) V]$ e $U[U(U V)] \oplus\left\{U^{4}+\mathrm{U}[(U V) V]\right\}$ são ideais de $A$, segue que o subespaço $\{(\mathrm{UV}) \mathrm{V}+\mathrm{U}[\mathrm{U}(\mathrm{UV})]\} \oplus\left\{\mathrm{U}^{4}+\right.$ $+\mathrm{U}[(\mathrm{UV}) \mathrm{V}]\}$ é um ideal de A, como soma de dois ideais.

Dessa forma, ficou provado que todos os subespaços do Grupo I são ideais de A. 


\subsection{Ideais gerados pelos monômios de Peirce}

Procuraremos agora, fixada uma t-álgebra $\mathrm{A}=\mathrm{Fe} \oplus \mathrm{U} \oplus \mathrm{V}$, de posto 3 , descrever os ideais gerados pelos $\mathrm{P}$-monômios não nulos de grau $\leq 4$ (numerados de 1 a 12 no Apêndice) e por alguns outros P-monômios, de grau não necessariamente 4. Em [20] os autores provaram que os ideais gerados por $\mathrm{U}$ e V são $\mathrm{U} \oplus \mathrm{U}^{2}$ e $\mathrm{UV} \oplus \mathrm{V}$, respectivamente.

Lema 6.1 Para todo $n \geq 1$, o ideal gerado por $U^{n}$ é o P-subespaço $U^{n} \oplus U^{n+1}$.

Prova: O subespaço vetorial $\mathrm{U}^{n} \oplus \mathrm{U}^{n+1}$, que contém $\mathrm{U}^{n}$, é ideal de A para todo $n \geq 1$ (vide Lema 2.4). Se $J$ é um outro ideal de A contendo $\mathrm{U}^{n}$, então $\mathrm{U}^{n+1}=\mathrm{U}^{n} \mathrm{U} \subseteq \mathrm{J} \mathrm{U} \subseteq \mathrm{J}$, pois $J$ é ideal de A e portanto $\mathrm{U}^{n} \oplus$ $\oplus \mathrm{U}^{n+1} \subseteq \mathrm{J}$, o que prova o resultado acima.

Lema 6.2 $O$ subespaço vetorial $U V \oplus U(U V)$ é o ideal gerado por $U V$.

Prova: Como UV $\oplus \mathrm{U}(\mathrm{UV})$ pertence ao Grupo I, do Teorema 1 sabemos que é um ideal de A e além disso contém UV. Considerando $J$ um outro ideal de A contendo UV, temos que $\mathrm{U}(\mathrm{UV}) \subseteq \mathrm{U} \mathrm{J} \subseteq \mathrm{J}$. Portanto UV $\ominus$ $\oplus \mathrm{U}(\mathrm{UV}) \subseteq \mathrm{J}$ e dessa forma conclui-se o resultado enunciado.

Lema 6.3 O ideal gerado por $U(U V)$ é o P-subespaço $U[U(U V)] \oplus$ $\oplus U(U V)$.

Prova: O subespaço vetorial $\mathrm{U}[\mathrm{U}(\mathrm{UV})] \oplus \mathrm{U}(\mathrm{UV})$, que contém $\mathrm{U}[\mathrm{U}(\mathrm{UV})]$, pertence ao Grupo I e portanto, pelo Teorema 1, é um ideal de A. Além disso, se $J$ é um outro ideal de A tal que $\mathrm{U}(\mathrm{UV}) \subseteq \mathrm{J}$, então $\mathrm{U}[\mathrm{U}(\mathrm{UV})] \subseteq$ $\subseteq \mathrm{U} \mathrm{J} \subseteq \mathrm{J}$. Logo $\mathrm{U}[\mathrm{U}(\mathrm{UV})] \oplus \mathrm{U}(\mathrm{UV}) \subseteq \mathrm{J}$, sendo, portanto, o ideal gerado por $\mathrm{U}(\mathrm{UV})$. 
Lema 6.4 O ideal gerado por $(U V) V$ é o P-subespaço $(U V) V \Theta$ $\oplus U[(U V) V]$.

Prova: (UV)V $\oplus \mathrm{U}[(\mathrm{UV}) \mathrm{V}]$ é ideal de A (contendo (UV)V) pois pertence ao Grupo I. Se $J$ é um outro ideal de A que contém (UV)V, então $\mathrm{U}[(\mathrm{UV}) \mathrm{V}] \subseteq \mathrm{U} \mathrm{J} \subseteq \mathrm{J}$ e isto completa a prova de que $(\mathrm{UV}) \mathrm{V} \oplus \mathrm{U}[(\mathrm{UV}) \mathrm{V}]$ é gerado por (UV)V.

Lema 6.5 O subespaço vetorial $p(U, V)=U[U(U V)] \oplus U\{U[U(U V)]\}$ é o ideal de A gerado por $U[U(U V)]$.

Prova: Mostremos, inicialmente, que $\mathrm{p}(\mathrm{U}, \mathrm{V})$ é um ideal de A. Como vimos no Teorema 1 , basta mostrarmos que $(u+v) p(U, V) \subseteq p(U, V)$, com $\mathrm{u} \in \mathrm{U}$ e $\mathrm{v} \in \mathrm{V}$. Como

$$
\begin{aligned}
& \mathrm{u}\{\mathrm{U}[\mathrm{U}(\mathrm{UV})]\} \subseteq \mathrm{U}\{\mathrm{U}[\mathrm{U}(\mathrm{UV})]\} \subseteq \mathrm{p}(\mathrm{U}, \mathrm{V}) \\
& \mathrm{u}\{\mathrm{U}\{\mathrm{U}[\mathrm{U}(\mathrm{UV})]\}\} \subseteq \mathrm{u}\left\{\mathrm{U}\left[\left(\mathrm{UU}^{2}\right)\right]\right\} \subseteq \mathrm{U}[\mathrm{U}(\mathrm{UV})] \subseteq \mathrm{p}(\mathrm{U}, \mathrm{V}) ; \\
& \mathrm{v}\{\mathrm{U}\{\mathrm{U}[\mathrm{U}(\mathrm{UV})]\}\} \subseteq \mathrm{v} \mathrm{U}^{4} \subseteq \mathrm{V}^{2}=0,
\end{aligned}
$$

resta apenas estudarmos $\mathrm{v}\{\mathrm{U}[\mathrm{U}(\mathrm{UV})]\}$. Para isso, considerando $\mathrm{u}_{3}\left[\mathrm{u}_{2}\left(\mathrm{u}_{1} \mathrm{v}_{1}\right)\right]$ um gerador de $\mathrm{U}[\mathrm{U}(\mathrm{UV})]$ e de $\mathrm{J}\left(\mathrm{v}, \mathrm{u}_{3}, \mathrm{u}_{2}\left(\mathrm{u}_{1} \mathrm{v}_{1}\right)\right)=0$, obtemos $\mathrm{v}\left\{\mathrm{u}_{3}\left[\mathrm{u}_{2}\left(\mathrm{u}_{1} \mathrm{v}_{1}\right)\right]\right\}=-\left(\mathrm{u}_{3} \mathrm{v}\right)\left[\mathrm{u}_{2}\left(\mathrm{u}_{1} \mathrm{v}_{1}\right)\right] \in(\mathrm{UV})[\mathrm{U}(\mathrm{UV})] \subseteq \mathrm{U}[\mathrm{U}(\mathrm{UV})] \subseteq$ $\subseteq \mathrm{p}(\mathrm{U}, \mathrm{V})$, pois $\mathrm{u}_{3}\left\{\mathrm{v}\left[\mathrm{u}_{2}\left(\mathrm{u}_{1} \mathrm{v}_{1}\right)\right]\right\}=0$. Portanto, por linearidade, segue que $\mathrm{v}\{\mathrm{U}[\mathrm{U}(\mathrm{UV})]\} \subseteq \mathrm{p}(\mathrm{U}, \mathrm{V})$ e assim conclui-se que $\mathrm{p}(\mathrm{U}, \mathrm{V})$ é um ideal de A que, obviamente, contém U[U(UV)]. Consideremos agora $J$ um outro ideal de A, contendo $\mathrm{U}[\mathrm{U}(\mathrm{UV})]$. Então $\mathrm{U}\{\mathrm{U}[\mathrm{U}(\mathrm{UV})]\} \subseteq \mathrm{U} \mathrm{J} \subseteq \mathrm{J}$, o que mostra o resultado enunciado.

Lema 6.6 O P-subespaço $p(U, V)=U\{U[(U V) V]\} \oplus U[(U V) V]$ éo ideal gerado por $U[(U V) V]$.

Prova: Afirmamos que $\mathrm{p}(\mathrm{U}, \mathrm{V})$ é um ideal de $\mathrm{A}$. De fato, se $\mathrm{u} \in \mathrm{U}$ e $\mathrm{v} \in \mathrm{V}$, então $\mathrm{u}\{\mathrm{U}[(\mathrm{UV}) \mathrm{V}]\} \subseteq \mathrm{U}\{\mathrm{U}[(\mathrm{UV}) \mathrm{V}]\} \subseteq \mathrm{p}(\mathrm{U}, \mathrm{V})$ e $\mathrm{v}\{\mathrm{U}[(\mathrm{UV}) \mathrm{V}]\} \subseteq \mathrm{VU}^{2}=$ $=0 \subseteq \mathrm{p}(\mathrm{U}, \mathrm{V})$. Resta, portanto, analisarmos os termos $\mathrm{u}\{\mathrm{U}\{\mathrm{U}[(\mathrm{UV}) \mathrm{V}]\}\}$ e $\mathrm{v}\{\mathrm{U}\{\mathrm{U}[(\mathrm{UV}) \mathrm{V}]\}\}$. Como $\mathrm{U}[(\mathrm{UV}) \mathrm{V}]=(\mathrm{UV})^{2}$ (Lema 3.1), um gerador 
de $U\{U[(U V) V]\}$ é da forma $u_{3}\left[\left(u_{1} v_{1}\right)\left(u_{2} v_{2}\right)\right]$. Considerando então os elementos $\mathrm{u}_{3}, \mathrm{u}_{1} \mathrm{v}_{1}$ e $\mathrm{u}_{2} \mathrm{v}_{2}$, obtemos $\mathrm{J}\left(\mathrm{u}_{3}, \mathrm{u}_{1} \mathrm{v}_{1}, \mathrm{u}_{2} \mathrm{v}_{2}\right)=\mathrm{u}_{3}\left[\left(\mathrm{u}_{1} \mathrm{v}_{1}\right)\left(\mathrm{u}_{2} \mathrm{v}_{2}\right)\right]+$ $+\left(\mathrm{u}_{1} \mathrm{v}_{1}\right)\left[\mathrm{u}_{3}\left(\mathrm{u}_{2} \mathrm{v}_{2}\right)\right]+\left(\mathrm{u}_{2} \mathrm{v}_{2}\right)\left[\mathrm{u}_{3}\left(\mathrm{u}_{1} \mathrm{v}_{1}\right)\right]=0$. Portanto $\mathrm{u}\left\{\mathrm{u}_{3}\left[\left(\mathrm{u}_{1} \mathrm{v}_{1}\right)\left(\mathrm{u}_{2} \mathrm{v}_{2}\right)\right]\right\}=$ $=-\mathrm{u}\left\{\left(\mathrm{u}_{1} \mathrm{v}_{1}\right)\left[\mathrm{u}_{3}\left(\mathrm{u}_{2} \mathrm{v}_{2}\right)\right]\right\}-\mathrm{u}\left\{\left(\mathrm{u}_{2} \mathrm{v}_{2}\right)\left[\mathrm{u}_{3}\left(\mathrm{u}_{1} \mathrm{v}_{1}\right)\right]\right\} \in \mathrm{U}\{(\mathrm{UV})[\mathrm{U}(\mathrm{UV})]\} \subseteq$ $\subseteq \mathrm{U}\left[(\mathrm{UV}) \mathrm{U}^{2}\right] \subseteq \mathrm{U}[(\mathrm{UV}) \mathrm{V}] \subseteq \mathrm{p}(\mathrm{U}, \mathrm{V})$ e $\operatorname{assim} \mathrm{u}\{\mathrm{U}\{\mathrm{U}[(\mathrm{UV}) \mathrm{V}]\}\} \subseteq$ $\subseteq \mathrm{p}(\mathrm{U}, \mathrm{V})$. Além disso, da identidade (1.13) aplicada aos elementos $\mathrm{v}, \mathrm{u}_{3},\left(\mathrm{u}_{1} \mathrm{v}_{1}\right)\left(\mathrm{u}_{2} \mathrm{v}_{2}\right)$, temos $\mathrm{v}\left\{\mathrm{u}_{3}\left[\left(\mathrm{u}_{1} \mathrm{v}_{1}\right)\left(\mathrm{u}_{2} \mathrm{v}_{2}\right)\right]\right\}=-\left(\mathrm{u}_{3} \mathrm{v}\right)\left[\left(\mathrm{u}_{1} \mathrm{v}_{1}\right)\left(\mathrm{u}_{2} \mathrm{v}_{2}\right)\right] \epsilon$ $\in(\mathrm{UV})(\mathrm{UV})^{2} \subseteq \mathrm{U}(\mathrm{UV})^{2}=\mathrm{U}\{\mathrm{U}[(\mathrm{UV}) \mathrm{V}]\} \subseteq \mathrm{p}(\mathrm{U}, \mathrm{V})$, e dessa forma $\mathrm{o}$ P-subespaço $\mathrm{p}(\mathrm{U}, \mathrm{V})$ é um ideal de A que, obviamente, contém o subespaço monomial U[(UV)V]. Consideremos agora $J$ um ideal de A contendo $\mathrm{U}[(\mathrm{UV}) \mathrm{V}]$. Então $\mathrm{U}\{\mathrm{U}[(\mathrm{UV}) \mathrm{V}]\} \subseteq \mathrm{U} \mathrm{J} \subseteq \mathrm{J}$ e portanto $\mathrm{p}(\mathrm{U}, \mathrm{V}) \subseteq \mathrm{J}$, o que mostra que $\mathrm{U}\{\mathrm{U}[(\mathrm{UV}) \mathrm{V}]\} \oplus \mathrm{U}[(\mathrm{UV}) \mathrm{V}]$ é o ideal de A gerado por $\mathrm{U}[(\mathrm{UV}) \mathrm{V}]$.

Lema 6.7 $O$ subespaço polinomial $U^{2 n+1} V \oplus U^{2 n+1}(U V)=U^{2 n+1} V \oplus$ $\oplus U\left(U^{2 n+1} V\right)$ é o ideal gerado por $U^{2 n+1} V$, para todo $n \geq 1$.

Prova: Inicialmente, mostremos que $\mathrm{U}^{2 n+1} \mathrm{~V} \oplus \mathrm{U}^{2 n+1}(\mathrm{UV})$ é um ideal de A, para todo $n \geq 1$. Tomemos $\mathrm{x}=\mathrm{u}+\mathrm{v} \in \mathrm{U}^{2 n+1} \mathrm{~V} \oplus \mathrm{U}^{2 n+1}(\mathrm{UV})$ e consideremos $u^{\prime} \in U$ e $v^{\prime} \in V$. Temos então que $\left(u^{\prime}+v^{\prime}\right) x=u^{\prime} u+u^{\prime} v+$ $+\mathrm{uv}^{\prime}+\mathrm{vv}^{\prime}=\mathrm{u}^{\prime} \mathrm{u}+\mathrm{u}^{\prime} \mathrm{v}+\mathrm{uv}^{\prime}$. Como $\mathrm{u}^{\prime} \mathrm{u} \in \mathrm{U}\left(\mathrm{U}^{2 n+1} \mathrm{~V}\right)=\mathrm{U}^{2 n+1}(\mathrm{UV})$ (pelo Lema 3.2, (iii)); v'x $=\mathrm{uv}^{\prime} \in\left(\mathrm{U}^{2 n+1} \mathrm{~V}\right) \mathrm{V}=\left[\mathrm{U}^{2 n}(\mathrm{UV})\right] \mathrm{V} \subseteq \mathrm{U}^{2 n+1} \mathrm{~V}$, (pelo Lema 3.2(ii)), resta apenas analisarmos o termo u'v. Mas u'v = $=\mathrm{u}^{\prime}\left[\left(\mathrm{tu}_{2}\right)\left(\mathrm{u}_{1} \mathrm{v}_{1}\right)\right]$, sendo $\mathrm{t} \in \mathrm{U}^{2 n}, \mathrm{u}_{1}, \mathrm{u}_{2} \in \mathrm{U}$ e $\mathrm{v}_{1} \in \mathrm{V}$ já que $\mathrm{v} \in$ $\in \mathrm{U}^{2 n+1}(\mathrm{UV})$. De J(u', tu $\left.\mathrm{u}_{2}, \mathrm{u}_{1} \mathrm{v}_{1}\right)=0$ temos $\mathrm{u}^{\prime} \mathrm{v}=-\left(\mathrm{tu}_{2}\right)\left[\mathrm{u}^{\prime}\left(\mathrm{u}_{1} \mathrm{v}_{1}\right)\right]+$ - $\left(\mathrm{u}_{1} \mathrm{v}_{1}\right)\left[\mathrm{u}^{\prime}\left(\mathrm{tu}_{2}\right)\right]$ e como $\left(\mathrm{tu}_{2}\right)\left[\mathrm{u}^{\prime}\left(\mathrm{u}_{1} \mathrm{v}_{1}\right)\right] \in \mathrm{U}^{2 n+1}[\mathrm{U}(\mathrm{UV})] \subseteq \mathrm{U}^{2 n+1} \mathrm{U}^{2} \subseteq$ $\subseteq \mathrm{U}^{2 n+1} \mathrm{~V}$, basta agora analisarmos o termo $\left(\mathrm{u}_{1} \mathrm{v}_{1}\right)\left[\mathrm{u}^{\prime}\left(\mathrm{tu}_{2}\right)\right]$. Usando-se agora a identidade (1.13) para os elementos $\mathrm{u}_{1}, \mathrm{v}_{1}, \mathrm{u}^{\prime}\left(\mathrm{tu}_{2}\right)$, obtemos $\mathrm{u}_{1}\left\{\mathrm{v}_{1}\left[\mathrm{u}^{\prime}\left(\mathrm{tu}_{2}\right)\right]\right\}+\mathrm{v}_{1}\left\{\mathrm{u}_{1}\left[\mathrm{u}^{\prime}\left(\mathrm{tu}_{2}\right)\right]\right\}+\left(\mathrm{u}_{1} \mathrm{v}_{1}\right)\left[\mathrm{u}^{\prime}\left(\mathrm{tu}_{2}\right)\right]=0$ e como $\mathrm{u}_{1}\left\{\mathrm{v}_{1}\left[\mathrm{u}^{\prime}\left(\mathrm{tu}_{2}\right)\right]\right\} \in \mathrm{U}\{\mathrm{V}[\mathrm{U}(\mathrm{UV})]\} \subseteq \mathrm{U}\left[\mathrm{VU}^{2}\right]=0$, temos $\left(\mathrm{u}_{1} \mathrm{v}_{1}\right)\left[\mathrm{u}^{\prime}\left(\mathrm{tu}_{2}\right)\right]=$ $=-\left\{\mathrm{u}_{1}\left[\mathrm{u}^{\prime}\left(\mathrm{tu}_{2}\right)\right]\right\} \mathrm{v}_{1} \in\left\{\mathrm{U}\left[\mathrm{U}\left(\mathrm{U}^{2 n} \mathrm{U}\right)\right]\right\} \mathrm{V}=\mathrm{U}^{2 n+3} \mathrm{~V} \subseteq \mathrm{U}^{2 n+1} \mathrm{~V}$, pelo Lema 2.1. Ou seja, $u^{\prime} v \in U^{2 n+1} V$ e portanto $\left(u^{\prime}+v^{\prime}\right) x \in U^{2 n+1} V \oplus U^{2 n+1}(U V)$, donde obtemos que $\mathrm{U}^{2 n+1} \mathrm{~V} \oplus \mathrm{U}^{2 n+1}(\mathrm{UV})$ é um ideal de A, para todo 
$n \geq 1$ e é claro que contém $\mathrm{U}^{2 n+1} \mathrm{~V}$. Além disso. se $J$ é um outro ideal de A contendo $\mathrm{U}^{2 n+1} \mathrm{~V}$, então $\mathrm{U}\left(\mathrm{U}^{2 n+1} \mathrm{~V}\right) \subseteq \mathrm{U} \mathrm{J} \subseteq \mathrm{J}$ e portanto obtemos o resultado enumciado.

Lema 6.8 O subespaço $U V^{(3)} \oplus U\left(U V^{(3)}\right)$ é o ideal gerado por $U V^{(3)}$.

Prova: Comecemos mostrando que $\mathrm{p}(\mathrm{U}, \mathrm{V})=[(\mathrm{UV}) \mathrm{V}] \mathrm{V} \oplus \mathrm{U}\{[(\mathrm{UV}) \mathrm{V}] \mathrm{V}\}$ é um ideal de A. Para isso, observemos que, se $\mathrm{u}+\mathrm{v} \in \mathrm{N}$, então $(\mathrm{u}+\mathrm{v})\{[(\mathrm{UV}) \mathrm{V}] \mathrm{V}\}=\mathrm{u}\{[(\mathrm{UV}) \mathrm{V}] \mathrm{V}\}+\mathrm{v}\{[(\mathrm{UV}) \mathrm{V}] \mathrm{V}\} \subseteq \mathrm{U}\{[(\mathrm{UV}) \mathrm{V}] \mathrm{V}\} \oplus$ $\oplus\{[(\mathrm{UV}) \mathrm{V}] \mathrm{V}\} \mathrm{V} \subseteq \mathrm{U}\{[(\mathrm{UV}) \mathrm{V}] \mathrm{V}\} \oplus[(\mathrm{UV}) \mathrm{V}] \mathrm{V}=\mathrm{p}(\mathrm{U}, \mathrm{V})$. Além disso, $\mathrm{v}\{\mathrm{U}\{[(\mathrm{UV}) \mathrm{V}] \mathrm{V}\}\} \subseteq \mathrm{VU}^{2} \subseteq \mathrm{V}^{2}=0$ e portanto resta apenas analisarmos o termo $u\{U\{[(U V) V] V\}\}$. Do Lema 3.2, (iv), segue que $U\{[(U V) V] V\}=$ $=(\mathrm{UV})[(\mathrm{UV}) \mathrm{V}]$ e assim um gerador de $\mathrm{U}\{[(\mathrm{UV}) \mathrm{V}] \mathrm{V}\}$ pode ser escrito na forma $\left(u_{1} v_{1}\right)\left[\left(u_{2} v_{2}\right) v\right]$. Assim, como $J\left(u, u_{1} v_{1},\left(u_{2} v_{2}\right) v\right)=0$, segue que $\mathrm{u}\left\{\left(\mathrm{u}_{1} \mathrm{v}_{1}\right)\left[\left(\mathrm{u}_{2} \mathrm{v}_{2}\right) \mathrm{v}\right]\right\}=-\left(\mathrm{u}_{1} \mathrm{v}_{1}\right)\left\{\mathrm{u}\left[\left(\mathrm{u}_{2} \mathrm{v}_{2}\right) \mathrm{v}\right]\right\}-\left[\left(\mathrm{u}_{2} \mathrm{v}_{2}\right) \mathrm{v}\right]\left[\mathrm{u}\left(\mathrm{u}_{1} \mathrm{v}_{1}\right)\right]$. Desse modo, $\left[\left(\mathrm{u}_{2} \mathrm{v}_{2}\right) \mathrm{v}\right]\left[\mathrm{u}\left(\mathrm{u}_{1} \mathrm{v}_{1}\right)\right] \in[(\mathrm{UV}) \mathrm{V}][\mathrm{U}(\mathrm{UV})] \subseteq[(\mathrm{UV}) \mathrm{V}] \mathrm{U}^{2} \subseteq[(\mathrm{UV}) \mathrm{V}] \mathrm{V} \subseteq$ $\subseteq \mathrm{p}(\mathrm{U}, \mathrm{V})$, e portanto resta analisarmos o termo $\mathrm{x}=\left(\mathrm{u}_{1} \mathrm{v}_{1}\right)\left\{\mathrm{u}\left[\left(\mathrm{u}_{2} \mathrm{v}_{2}\right) \mathrm{v}\right]\right\}$. Aplicando-se a identidade de Jacobi aos elementos $\mathrm{u}_{1}, \mathrm{v}_{1}, \mathrm{u}\left[\left(\mathrm{u}_{2} \mathrm{v}_{2}\right) \mathrm{v}\right]$, obte$\operatorname{mos} \mathrm{x}=-\left\{\mathrm{u}_{1}\left\{\mathrm{u}\left[\left(\mathrm{u}_{2} \mathrm{v}_{2}\right) \mathrm{v}\right]\right\}\right\} \mathrm{v}_{1}$, pois $\mathrm{u}_{1}\left\{\left\{\mathrm{u}\left[\left(\mathrm{u}_{2} \mathrm{v}_{2}\right) \mathrm{v}\right]\right\} \mathrm{v}_{1}\right\} \in \mathrm{U}\left[\mathrm{U}^{2} \mathrm{~V}\right]=0$. Da identidade (4.4) aplicada aos elementos $\mathrm{u}, \mathrm{u}_{2} \mathrm{v}_{2}, \mathrm{v}$, segue que $\mathrm{u}\left[\left(\mathrm{u}_{2} \mathrm{v}_{2}\right) \mathrm{v}\right]=$ $=-\left(\mathrm{u}_{2} \mathrm{v}_{2}\right)(\mathrm{uv})$ e assim $\mathrm{x}=\left\{\mathrm{u}_{1}\left[\left(\mathrm{u}_{2} \mathrm{v}_{2}\right)(\mathrm{uv})\right]\right\} \mathrm{v}_{1}$. Finalmente, da identidade (1.13) aplicada aos termos $\mathrm{u}_{1}, \mathrm{u}_{2} \mathrm{v}_{2}$, uv, segue que $\mathrm{u}_{1}\left[\left(\mathrm{u}_{2} \mathrm{v}_{2}\right)(\mathrm{uv})\right]=$ $=-\left(\mathrm{u}_{2} \mathrm{v}_{2}\right)\left[\mathrm{u}_{1}(\mathrm{uv})\right]-(\mathrm{uv})\left[\mathrm{u}_{1}\left(\mathrm{u}_{2} \mathrm{v}_{2}\right)\right] \in(\mathrm{UV})[\mathrm{U}(\mathrm{UV})] \subseteq(\mathrm{UV}) \mathrm{U}^{2} \subseteq(\mathrm{UV}) \mathrm{V}$. Logo, $\mathrm{x} \in[(\mathrm{UV}) \mathrm{V}] \mathrm{v}_{1} \subseteq[(\mathrm{UV}) \mathrm{V}] \mathrm{V}$ e portanto $\mathrm{u}\{\mathrm{U}\{[(\mathrm{UV}) \mathrm{V}] \mathrm{V}\}\} \subseteq$ $\subseteq \mathrm{p}(\mathrm{U}, \mathrm{V})$, o que mostra que $\mathrm{p}(\mathrm{U}, \mathrm{V})$ é um ideal de $\mathrm{A}$. Além disso, se $J$ é outro ideal de A contendo o subespaço monomial [(UV)V]V, então $\mathrm{U}\{[(\mathrm{UV}) \mathrm{V}] \mathrm{V}\} \subseteq \mathrm{U} \mathrm{J} \subseteq \mathrm{J}$ e com isto completamos a prova do resultado enunciado.

Observemos agora que se $\mathrm{B}$ e $\mathrm{C}$ são subespaços vetoriais de um mesmo espaço vetorial $A$ que geram, respectivamente, os ideais $\mathrm{I}_{B}$ e $\mathrm{I}_{C}$, então o ideal gerado por $\mathrm{B}+\mathrm{C}$ é $\mathrm{I}_{B}+\mathrm{I}_{C}$. Dessa forma, obteremos o seguinte resultado: 
Corolário 6.8.1 Os ideais gerados pelos P-subespaços $U^{3}+(U V) \mathrm{T}$. $U^{3}+[(U V) V] V,(U V) V+U[U(U V)], U^{4}+U[(U V) V], U[U(U V)]+$ $+[(U V) V] V, U^{3} V+[(U V) V] V$ são respectivamente dados por:

$$
\begin{gathered}
{\left[U^{3}+(U V) V\right] \oplus\left\{U^{4}+U[(U V) V]\right\} ;} \\
\left\{U^{3}+[(U V) V] V\right\} \oplus\left\{U^{4}+U\{[(U V) V] V\}\right\} ; \\
\{(U V) V+U[U(U V)]\} \oplus\{U[(U V) V]+U\{[U(U V)]\}\} ; \\
\left\{U^{5}+U\{U[(U V) V]\}\right\} \oplus\left\{U^{4}+U[(U V) V]\right\} ; \\
\{U[U(U V)]+[(U V) V] V\} \oplus\{U\{U[U(U V)]\}+U\{[(U V) V] V\}\} ; \\
\left\{U^{3} V+[(U V) V] V\right\} \oplus\left\{U^{3}(U V)+U\{[(U V) V] V\}\right\} .
\end{gathered}
$$

\subsection{Propriedades de ideais}

Lema 6.9 Se I é um ideal de uma t-álgebra de posto $3 \mathrm{~A}=\mathrm{Fe} \oplus U \oplus$ $\oplus V$, contido em $N$, então $I=(I \cap U) \oplus(I \cap V)$.

Prova: Considermos $\mathrm{x} \in \mathrm{I}$ um elemento arbitrário de $\mathrm{I}$. Como $\mathrm{I} \subseteq \mathrm{N}$, segue que $\mathrm{x}=\mathrm{u}+\mathrm{v}$, com $\mathrm{u} \in \mathrm{U}$ e $\mathrm{v} \in \mathrm{V}$. Portanto, $2 \mathrm{ex}=2 \mathrm{eu}+2 \mathrm{ev}=$ $=\mathrm{u}+2 \gamma \mathrm{v} \in \mathrm{I}$, pois I é ideal de A. Da mesma forma, 2ex - $\mathrm{x}=$ $=(2 \gamma-1) \mathrm{v} \in \mathrm{I}$ e como $2 \gamma \neq 1$, segue que $\mathrm{v} \in \mathrm{I}$. Assim obtemos que $\mathrm{v} \in \mathrm{I} \cap \mathrm{V}$. Finalmente, $\mathrm{u}=\mathrm{x}-\mathrm{v} \in \mathrm{I}$ e portanto $\mathrm{u} \in \mathrm{I} \cap \mathrm{U}$, o que conclui a demonstração do resultado enunciado.

Lema 6.10 Se I e J são ideais de uma t-álgebra $A=F e \oplus U \oplus V$, de posto 3, contidos em $N$, entâo $I J=\left\{\sum_{i} a_{i} b_{i}: a_{i} \in I, b_{i} \in J\right\}$ também. é um ideal de $A$.

Prova: É claro que I J é um subespaço vetorial de A. Consideremos agora $i j, \mathrm{i} \in \mathrm{I}, \mathrm{j} \in \mathrm{J}$, um gerador de I J. Pelo Lema 6.9, $\mathrm{i}=\mathrm{u}_{1}+\mathrm{v}_{1}$, $\mathrm{j}=\mathrm{u}_{2}+\mathrm{v}_{2}$, com $\mathrm{u}_{1} \in \mathrm{I} \cap \mathrm{U}, \mathrm{u}_{2} \in J \cap \mathrm{U}, \mathrm{v}_{1} \in \mathrm{I} \cap \mathrm{U}$ e $\mathrm{v}_{2} \in \mathrm{J} \cap \mathrm{V}$ e portanto $\mathrm{i} j=\mathrm{u}_{1} \mathrm{v}_{2}+\mathrm{u}_{2} \mathrm{v}_{1}+\mathrm{u}_{1} \mathrm{u}_{2}$, donde segue que $2 \mathrm{e}(\mathrm{ij})=\mathrm{u}_{1} \mathrm{v}_{2}+$ $+\mathrm{u}_{2} \mathrm{v}_{1}+2 \gamma \mathrm{u}_{1} \mathrm{u}_{2} \in \mathrm{I} \mathrm{J}$, pois cada parcela dessa soma está em I J. Além disso, dado $\mathrm{x} \in \mathrm{N}$, usando-se a identidade de Jacobi para os elementos $\mathrm{x}$. 
$\mathrm{i}, \mathrm{j}$, obtemos $\mathrm{x}(\mathrm{ij})=-\mathrm{i}(\mathrm{xj})-\mathrm{j}(\mathrm{xi}) \in \mathrm{I}(\mathrm{NJ})+\mathrm{J}(\mathrm{NI}) \subseteq \mathrm{I} J$, pois I. J são ideais de $\mathrm{A}$ e isto completa a prova de que I $\mathrm{J}$ é um ideal de $\mathrm{A}$.

Corolário 6.10.1 O produto de dois subespaços do Grupo I é um ideal de A.

Prova: De acordo com o Teorema 1, cada subespaço do Grupo I é um ideal de A e portanto, pelo Lema 6.10, segue o resultado.

Observação: A recíproca do lema anterior não é verdadeira, pois $\mathrm{NU}=$ $=(\mathrm{U} \oplus \mathrm{V}) \mathrm{U}=\mathrm{UV} \oplus \mathrm{U}^{2}=\mathrm{N}^{2}$, que é ideal de A. Além disso, $\mathrm{U}$ não é ideal de A, por (1.8).

Queremos, agora, saber quais dos 65 subespaços do Grupo II são ideais de A. Nesse caso, acreditamos que, genericamente, nenhum dos subespaços seja ideal de A, mas para podermos afirmar que isto ocorre, devemos dar contra-exemplos que mostrem que a afirmação é verdadeira.

Exemplo 27: Retomemos a álgebra A do Exemplo 22 do Capítulo anterior, cuja tábua de multiplicação é:

$$
\left\{\begin{array}{l}
\mathrm{e}^{2}=\mathrm{e} ; 2 \mathrm{eu}_{\mathrm{i}}=\mathrm{u}_{\mathrm{i}}(\mathrm{i}=1, \ldots, 12) ; \quad \mathrm{v}_{\mathrm{i}}=0(\mathrm{i}=1, \ldots, 7) \\
\mathrm{u}_{2}^{2}=\mathrm{v}_{3} ; \mathrm{u}_{1} \mathrm{u}_{2}=\mathrm{v}_{4} ;-\mathrm{u}_{1} \mathrm{u}_{5}=\mathrm{u}_{2} \mathrm{u}_{3}=\mathrm{v}_{5} ; \quad \mathrm{u}_{1} \mathrm{u}_{4}=-\mathrm{u}_{2} \mathrm{u}_{6}=\mathrm{v}_{6} \\
\mathrm{u}_{1} \mathrm{u}_{7}=\mathrm{u}_{2} \mathrm{u}_{8}=-\mathrm{u}_{3} \mathrm{u}_{4}=\mathrm{u}_{5} \mathrm{u}_{6}=-\mathrm{v}_{7} ; \quad \mathrm{u}_{1} \mathrm{v}_{1}=\mathrm{u}_{3} ; \quad \mathrm{u}_{1} \mathrm{v}_{2}=\mathrm{u}_{6} \\
\mathrm{u}_{2} \mathrm{v}_{1}=\mathrm{u}_{5} ; \quad \mathrm{u}_{2} \mathrm{v}_{2}=\mathrm{u}_{4} ; \quad \mathrm{u}_{4} \mathrm{v}_{1}=-\mathrm{u}_{5} \mathrm{v}_{2}=\mathrm{u}_{7} ; \quad \mathrm{u}_{3} \mathrm{v}_{2}=-\mathrm{u}_{6} \mathrm{v}_{1}=\mathrm{u}_{8} \\
\mathrm{u}_{1} \mathrm{v}_{3}=-2 \mathrm{u}_{2} \mathrm{v}_{4}=\mathrm{u}_{9} ;-2 \mathrm{u}_{2} \mathrm{v}_{5}=\mathrm{u}_{3} \mathrm{v}_{3}=-2 \mathrm{u}_{5} \mathrm{v}_{4}=-\mathrm{u}_{9} \mathrm{v}_{1}=\mathrm{u}_{10} \\
2 \mathrm{u}_{2} \mathrm{v}_{6}=-2 \mathrm{u}_{4} \mathrm{v}_{4}=\mathrm{u}_{6} \mathrm{v}_{3}=-\mathrm{u}_{9} \mathrm{v}_{2}=\mathrm{u}_{11} \\
2 \mathrm{u}_{2} \mathrm{v}_{7}=-2 \mathrm{u}_{4} \mathrm{v}_{5}=-2 \mathrm{u}_{5} \mathrm{v}_{6}=2 \mathrm{u}_{7} \mathrm{v}_{4}=\mathrm{u}_{8} \mathrm{v}_{3}=-\mathrm{u}_{10} \mathrm{v}_{2}=\mathrm{u}_{11} \mathrm{v}_{1}=\mathrm{u}_{12} \\
\text { os outros produtos são nulos }
\end{array}\right.
$$

É fácil ver que os $\mathrm{P}$-monômios $\mathrm{U}, \mathrm{UV}=\mathrm{Fu}_{3} \oplus \ldots \oplus \mathrm{Fu}_{12},(\mathrm{UV}) \mathrm{V}=$ 
$=\mathrm{Fu}_{7} \oplus \mathrm{Fu}_{8} \oplus \mathrm{Fu}_{10} \oplus \mathrm{Fu}_{11} \oplus \mathrm{Fu}_{12}$ não são ideais de $\mathrm{A}$, uma vez que $\mathrm{u}_{2} \mathrm{u}_{8}=-\mathrm{v}_{7}$. Por outro lado, $\mathrm{V}, \mathrm{U}^{2}=\mathrm{Fv}_{4} \oplus \ldots \oplus \mathrm{Fv}_{7}, \mathrm{U}(\mathrm{UV})=\mathrm{Fv}_{5} \oplus$ $\oplus \mathrm{Fv}_{6} \oplus \mathrm{Fv}_{7}$ e $\mathrm{U}[(\mathrm{UV}) \mathrm{V}]=\mathrm{Fv}_{7}$ não são ideais de $\mathrm{A}$ pois $2 \mathrm{u}_{2} \mathrm{v}_{7}=\mathrm{u}_{12}$. Além disso, os P-monômios $\mathrm{U}^{3}=\mathrm{Fu}_{9} \oplus \ldots \oplus \mathrm{Fu}_{12}, \mathrm{U}[\mathrm{U}(\mathrm{UV})]=\mathrm{U}^{3} \mathrm{~V}=$ $=\mathrm{Fu}_{10} \oplus \mathrm{Fu}_{11} \oplus \mathrm{Fu}_{12},[(\mathrm{UV}) \mathrm{V}] \mathrm{V}=\mathrm{Fu}_{12}$ e $\mathrm{U}^{4}=0$ são ideais de A. Assim, o seguinte P-subespaço do Grupo II não é ideal de A:

$$
\text { 10. } \mathrm{U}^{4}+\mathrm{U}[(\mathrm{UV}) \mathrm{V}]=\mathrm{U}[(\mathrm{UV}) \mathrm{V}]
$$

Exemplo 28: Consideremos a t-álgebra A dada no Exemplo 26 do Capítulo 5. Sua tábua de multiplicação é dada por

$$
\left\{\begin{array}{l}
\mathrm{e}^{2}=\mathrm{e} ; \quad 2 \mathrm{u}_{\mathrm{i}}=\mathrm{u}_{\mathrm{i}}(\mathrm{i}=1, \ldots, 16) ; \quad \text { evi }=0(\mathrm{i}=1, \ldots, 9) \\
\mathrm{u}_{1}^{2}=\mathrm{v}_{3} ; \mathrm{u}_{2}^{2}=\mathrm{v}_{4} ; \quad \mathrm{u}_{1} \mathrm{u}_{2}=\mathrm{v}_{5} \\
\mathrm{u}_{1} \mathrm{u}_{5}=-\mathrm{u}_{2} \mathrm{u}_{3}=-\mathrm{v}_{6} ; \quad \mathrm{u}_{1} \mathrm{u}_{6}=-\mathrm{u}_{2} \mathrm{u}_{4}=\mathrm{v}_{7} \\
\mathrm{u}_{1} \mathrm{u}_{9}=\mathrm{u}_{2} \mathrm{u}_{10}=\mathrm{u}_{3} \mathrm{u}_{6}=-\mathrm{u}_{4} \mathrm{u}_{5}=-\mathrm{v}_{8} \\
\mathrm{u}_{1} \mathrm{u}_{16}=\mathrm{u}_{2} \mathrm{u}_{15}=\mathrm{u}_{3} \mathrm{u}_{14}=\mathrm{u}_{7} \mathrm{u}_{9}=\mathrm{v}_{9} \\
\mathrm{u}_{4} \mathrm{u}_{11}=\mathrm{u}_{5} \mathrm{u}_{12}=\mathrm{u}_{6} \mathrm{u}_{13}=\mathrm{u}_{8} \mathrm{u}_{10}=-\mathrm{v}_{9} \\
\mathrm{u}_{1} \mathrm{v}_{1}=\mathrm{u}_{3} ; \quad \mathrm{u}_{1} \mathrm{v}_{2}=\mathrm{u}_{4} ; \quad \mathrm{u}_{2} \mathrm{v}_{1}=\mathrm{u}_{5} ; \quad \mathrm{u}_{2} \mathrm{v}_{2}=\mathrm{u}_{6} \\
2 \mathrm{u}_{1} \mathrm{v}_{5}=-\mathrm{u}_{2} \mathrm{v}_{3}=-\mathrm{u}_{7} ; \quad \mathrm{u}_{1} \mathrm{v}_{4}=2 \mathrm{u}_{2} \mathrm{v}_{5}=-\mathrm{u}_{8} \\
\mathrm{u}_{5} \mathrm{v}_{2}=-\mathrm{u}_{6} \mathrm{v}_{1}=\mathrm{u}_{9} ; \quad \mathrm{u}_{3} \mathrm{v}_{2}=-\mathrm{u}_{4} \mathrm{v}_{1}=-\mathrm{u}_{10} \\
2 \mathrm{u}_{2} \mathrm{v}_{6}=-\mathrm{u}_{3} \mathrm{v}_{4}=2 \mathrm{u}_{5} \mathrm{v}_{5}=\mathrm{u}_{8} \mathrm{v}_{1}=-\mathrm{u}_{11} \\
2 \mathrm{u}_{1} \mathrm{v}_{7}=2 \mathrm{u}_{4} \mathrm{v}_{5}=-\mathrm{u}_{6} \mathrm{v}_{3}=\mathrm{u}_{7} \mathrm{v}_{2}=\mathrm{u}_{12} \\
2 \mathrm{u}_{1} \mathrm{v}_{6}=-2 \mathrm{u}_{3} \mathrm{v}_{5}=\mathrm{u}_{5} \mathrm{v}_{3}=-\mathrm{u}_{7} \mathrm{v}_{1}=\mathrm{u}_{13} \\
2 \mathrm{u}_{2} \mathrm{v}_{7}=\mathrm{u}_{4} \mathrm{v}_{4}=-2 \mathrm{u}_{6} \mathrm{v}_{5}=-\mathrm{u}_{8} \mathrm{v}_{2}=\mathrm{u}_{14} \\
2 \mathrm{u}_{1} \mathrm{v}_{8}=2 \mathrm{u}_{3} \mathrm{v}_{7}=2 \mathrm{u}_{4} \mathrm{v}_{6}=\mathrm{u}_{9} \mathrm{v}_{3}=2 \mathrm{u}_{10} \mathrm{v}_{5}=-\mathrm{u}_{12} \mathrm{v}_{1}=-\mathrm{u}_{13} \mathrm{v}_{2}=-\mathrm{u}_{15} \\
2 \mathrm{u}_{2} \mathrm{v}_{8}=2 \mathrm{u}_{5} \mathrm{v}_{7}=2 \mathrm{u}_{6} \mathrm{v}_{6}=2 \mathrm{u}_{9} \mathrm{v}_{5}=\mathrm{u}_{10} \mathrm{v}_{4}=\mathrm{u}_{11} \mathrm{v}_{2}=-\mathrm{u}_{14} \mathrm{v}_{1}=\mathrm{u}_{16} \\
\text { os outros produtos são nulos }
\end{array}\right.
$$

É fácil ver que $\mathrm{U}[\mathrm{U}(\mathrm{UV})]=\mathrm{U}^{3} \mathrm{~V}=\mathrm{F}_{11} \oplus \ldots \oplus \mathrm{F}_{16}$. Além disso, como $\mathrm{u}_{1} \mathrm{u}_{16}=\mathrm{v}_{9} \notin \mathrm{U}[\mathrm{U}(\mathrm{UV})]=\mathrm{U}^{3} \mathrm{~V}$, segue que estes dois $\mathrm{P}$-monômios não são ideais de $\mathrm{A}$. 
Exemplo 29: Retomemos a t-álgebra A de posto 3 dada no Exemplo 24 do Capítulo 5, cuja tábua de multiplicação é dada por:

$$
\left\{\begin{array}{l}
\mathrm{e}^{2}=\mathrm{e} ; 2 \mathrm{eu}_{\mathrm{i}}=\mathrm{u}_{\mathrm{i}}(\mathrm{i}=1, \ldots, 11) ; \quad \text { ev } \mathrm{i}=0(\mathrm{i}=1, \ldots, 8) \\
\mathrm{u}_{1}^{2}=\mathrm{v}_{1} ; \quad \mathrm{u}_{3}^{2}=-\mathrm{v}_{1}+2 \mathrm{v}_{3} ; \quad \mathrm{u}_{1} \mathrm{u}_{2}=\mathrm{u}_{2} \mathrm{u}_{3}=\mathrm{v}_{2} ; \quad \mathrm{u}_{1} \mathrm{u}_{3}=\mathrm{v}_{3} \\
2 \mathrm{u}_{2} \mathrm{u}_{10}=-\mathrm{v}_{6}+2 \mathrm{v}_{8} ; \mathrm{u}_{1} \mathrm{u}_{4}=\mathrm{u}_{3} \mathrm{u}_{4}=\mathrm{v}_{4} ;-\mathrm{u}_{3} \mathrm{u}_{9}=\mathrm{u}_{4} \mathrm{u}_{8}=\mathrm{v}_{8} \\
2 \mathrm{u}_{1} \mathrm{u}_{8}=-\mathrm{u}_{2} \mathrm{u}_{6}=\mathrm{u}_{3} \mathrm{u}_{5}=\mathrm{v}_{5} ; 2 \mathrm{u}_{1} \mathrm{u}_{9}=-\mathrm{u}_{2} \mathrm{u}_{7}=\mathrm{u}_{4} \mathrm{u}_{5}=\mathrm{v}_{6} \\
-2 \mathrm{u}_{1} \mathrm{v}_{2}=\mathrm{u}_{2} \mathrm{v}_{1}=\mathrm{u}_{5} ;-2 \mathrm{u}_{1} \mathrm{v}_{4}=\mathrm{u}_{4} \mathrm{v}_{1}=\mathrm{u}_{7} \\
2 \mathrm{u}_{1} \mathrm{u}_{10}=-\mathrm{u}_{3} \mathrm{u}_{7}=-2 \mathrm{u}_{3} \mathrm{u}_{10}=\mathrm{u}_{4} \mathrm{u}_{6}=\mathrm{v}_{7} \\
-2 \mathrm{u}_{1} \mathrm{v}_{3}=\mathrm{u}_{3} \mathrm{v}_{1}=2 \mathrm{u}_{3} \mathrm{v}_{3}=\mathrm{u}_{6} ; \mathrm{u}_{3} \mathrm{v}_{2}=\mathrm{u}_{8} ;-\mathrm{u}_{2} \mathrm{v}_{4}=\mathrm{u}_{4} \mathrm{v}_{2}=\mathrm{u}_{9} \\
\mathrm{u}_{4} \mathrm{v}_{3}=\mathrm{u}_{10} ;-2 \mathrm{u}_{1} \mathrm{v}_{8}=\mathrm{u}_{2} \mathrm{v}_{7}=-\mathrm{u}_{3} \mathrm{v}_{6}=\mathrm{u}_{4} \mathrm{v}_{5}=\mathrm{u}_{11} \\
2 \mathrm{u}_{2} \mathrm{v}_{3}=\mathrm{u}_{5}-2 \mathrm{u}_{8} ; 2 \mathrm{u}_{3} \mathrm{v}_{4}=\mathrm{u}_{7}-2 \mathrm{u}_{10} \\
\text { os outros produtos são nulos }
\end{array}\right.
$$

Da tábua de multiplicação, vemos que os seguintes $\mathrm{P}$-monômios não são ideais de $\mathrm{A}: \mathrm{U}^{2}=\mathrm{V}$ e $\mathrm{U}(\mathrm{UV})=\mathrm{U}^{4}=\mathrm{Fv}_{5} \oplus \ldots \oplus \mathrm{Fv}_{8}\left(\right.$ pois $\mathrm{u}_{2} \mathrm{v}_{7}=$ $=\mathrm{u}_{11}$ ); $\mathrm{U}$ e $\mathrm{UV}=\mathrm{U}^{3}=\mathrm{Fu}_{5} \oplus \ldots \oplus \mathrm{Fu}_{11}$ (pois $\mathrm{u}_{4} \mathrm{u}_{5}=\mathrm{u}_{11}$ ). Além disso, os $\mathrm{P}$-monômios $\mathrm{U}[\mathrm{U}(\mathrm{UV})]=\mathrm{Fu}_{11},(\mathrm{UV}) \mathrm{V}=\mathrm{U}[(\mathrm{UV}) \mathrm{V}]=[(\mathrm{UV}) \mathrm{V}] \mathrm{V}=$ $=\mathrm{U}^{3} \mathrm{~V}=0$ são ideais de A. Com isto, fica mostrado que os seguintes P-subespaços do Grupo II não são ideais de A:

1. U

2. $\mathrm{V}$

3. $\mathrm{U}^{2}$

4. UV

5. $\mathrm{U}^{3}$

6. U(UV)

8. $\mathrm{U}^{4}$

14. $\mathrm{U} \oplus \mathrm{U}(\mathrm{UV})$, pois $\mathrm{u}_{1} \mathrm{u}_{4} \notin \mathrm{U}(\mathrm{UV})$

15. $\mathrm{U} \oplus \mathrm{U}^{4}=\mathrm{U} \oplus \mathrm{U}(\mathrm{UV})$

16. $\mathrm{U} \oplus \mathrm{U}[(\mathrm{UV}) \mathrm{V}]=\mathrm{U}$

19. $(\mathrm{UV}) \mathrm{V} \oplus \mathrm{V}=\mathrm{V}$

20. $\mathrm{U}[\mathrm{U}(\mathrm{UV})] \ominus \mathrm{V}$, pois $\mathrm{u}_{2} \mathrm{v}_{1} \notin \mathrm{U}[\mathrm{U}(\mathrm{UV})]$ 
21. $\mathrm{U}^{3} \mathrm{~V} \oplus \mathrm{V}=\mathrm{V}$

22. $[(\mathrm{UV}) \mathrm{V}] \mathrm{V} \oplus \mathrm{V}=\mathrm{V}$

25. $(\mathrm{UV}) \mathrm{V} \oplus \mathrm{U}^{2}=\mathrm{U}^{2}=\mathrm{V}$

26. $\mathrm{U}[\mathrm{U}(\mathrm{UV})] \oplus \mathrm{U}^{2}=\mathrm{U}[\mathrm{U}(\mathrm{UV})] \oplus \mathrm{V}$ (vide 20$)$

27. $\mathrm{U}^{3} \mathrm{~V} \oplus \mathrm{U}^{2}=\mathrm{U}^{2}=\mathrm{V}$

28. $[(\mathrm{UV}) \mathrm{V}] \mathrm{V} \oplus \mathrm{U}^{2}=\mathrm{U}^{2}=\mathrm{V}$

31. $\mathrm{UV} \oplus \mathrm{U}[(\mathrm{UV}) \mathrm{V}]=\mathrm{UV}$

33. $\mathrm{U}^{3}+(\mathrm{UV}) \mathrm{V}=\mathrm{U}^{3}$

35. $\mathrm{U}^{3} \oplus \mathrm{U}[(\mathrm{UV}) \mathrm{V}]=\mathrm{U}^{3}$

36. $\mathrm{U}^{3}+[(\mathrm{UV}) \mathrm{V}] \mathrm{V}=\mathrm{U}^{3}$

37. $(\mathrm{UV}) \mathrm{V} \oplus \mathrm{U}(\mathrm{UV})=\mathrm{U}(\mathrm{UV})$

39. $\mathrm{U}^{3} \mathrm{~V} \oplus \mathrm{U}(\mathrm{UV})=\mathrm{U}(\mathrm{UV})$

40. $[(\mathrm{UV}) \mathrm{V}] \mathrm{V} \oplus \mathrm{U}(\mathrm{UV})=\mathrm{U}(\mathrm{UV})$

41. $(\mathrm{UV}) \mathrm{V} \oplus \mathrm{U}^{4}=\mathrm{U}^{4}$

45. $\mathrm{U}^{4}+\mathrm{U}[(\mathrm{UV}) \mathrm{V}]=\mathrm{U}^{4}$

46. $\mathrm{U}^{3} \mathrm{~V} \oplus \mathrm{U}^{4}=\mathrm{U}^{4}$

47. $[(\mathrm{UV}) \mathrm{V}] \mathrm{V} \oplus \mathrm{U}^{4}=\mathrm{U}^{4}$

53. $\mathrm{U} \oplus\left\{\mathrm{U}^{4}+\mathrm{U}[(\mathrm{UV}) \mathrm{V}]\right\}=\mathrm{U} \oplus \mathrm{U}^{4}($ veja 15$)$

56. $\{(\mathrm{UV}) \mathrm{V}+\mathrm{U}[\mathrm{U}(\mathrm{UV})]\} \oplus \mathrm{V}=\mathrm{U}[\mathrm{U}(\mathrm{UV})] \oplus \mathrm{V}($ veja 20$)$

57. $\{\mathrm{U}[\mathrm{U}(\mathrm{UV})]+[(\mathrm{UV}) \mathrm{V}] \mathrm{V}\} \oplus \mathrm{V}=\mathrm{U}[\mathrm{U}(\mathrm{UV})] \oplus \mathrm{V}($ veja 20$)$

58. $\left\{\mathrm{U}^{3} \mathrm{~V}+[(\mathrm{UV}) \mathrm{V}] \mathrm{V}\right\} \oplus \mathrm{V}=\mathrm{V}$

61. $\{(\mathrm{UV}) \mathrm{V}+\mathrm{U}[\mathrm{U}(\mathrm{UV})]\} \oplus \mathrm{U}^{2}=\mathrm{U}[\mathrm{U}(\mathrm{UV})] \oplus \mathrm{U}^{2}$ (veja 26)

62. $\{\mathrm{U}[\mathrm{U}(\mathrm{UV})]+[(\mathrm{UV}) \mathrm{V}] \mathrm{V}\} \oplus \mathrm{U}^{2}=\mathrm{U}[\mathrm{U}(\mathrm{UV})] \oplus \mathrm{U}^{2}(\operatorname{veja} 26)$

63. $\left\{\mathrm{U}^{3} \mathrm{~V}+[(\mathrm{UV}) \mathrm{V}] \mathrm{V}\right\} \oplus \mathrm{U}^{2}=\mathrm{U}^{2}$

68. $\left[\mathrm{U}^{3}+(\mathrm{UV}) \mathrm{V}\right] \oplus \mathrm{U}[(\mathrm{UV}) \mathrm{V}]=\mathrm{U}^{3}$

71. $\left\{\mathrm{U}^{3}+[(\mathrm{UV}) \mathrm{V}] \mathrm{V}\right\} \oplus \mathrm{U}[(\mathrm{UV}) \mathrm{V}]=\mathrm{U}^{3}$

74. $\left\{\mathrm{U}^{3} \mathrm{~V}+[(\mathrm{UV}) \mathrm{V}] \mathrm{V}\right\} \oplus \mathrm{U}(\mathrm{UV})=\mathrm{U}(\mathrm{UV})$

76. $(\mathrm{UV}) \mathrm{V} \oplus\left\{\mathrm{U}^{4}+\mathrm{U}[(\mathrm{UV}) \mathrm{V}]\right\}=\mathrm{U}^{4}$

80. $\mathrm{U}^{3} \mathrm{~V} \oplus\left\{\mathrm{U}^{4}+\mathrm{U}[(\mathrm{UV}) \mathrm{V}]\right\}=\mathrm{U}^{4}$

81. $[(\mathrm{UV}) \mathrm{V}] \mathrm{V} \oplus\left\{\mathrm{U}^{-4}+\mathrm{U}[(\mathrm{UV}) \mathrm{V}]\right\}=\mathrm{U}^{4}$

82. $\left\{\mathrm{U}^{3} \mathrm{~V}+[(\mathrm{UV}) \mathrm{V}] \mathrm{V}\right\} \oplus \mathrm{U}^{4}=\mathrm{U}^{4}$

89. $\left\{\mathrm{U}^{3} \mathrm{~V}+[(\mathrm{UV}) \mathrm{V}] \mathrm{V}\right\} \oplus\left\{\mathrm{U}^{4}+\mathrm{U}[(\mathrm{UV}) \mathrm{V}]\right\}=\mathrm{U}^{4}$ 
Exemplo 30: Consideremos a t-álgebra A dada no Exemplo 25 do Capítulo 5. Sua tábua de multiplicação é dada por

$$
\left\{\begin{array}{l}
\mathrm{e}^{2}=\mathrm{e} ; 2 \mathrm{eu}_{\mathrm{i}}=\mathrm{u}_{\mathrm{i}}(\mathrm{i}=1, \ldots, 16) ; \quad \mathrm{v}_{\mathrm{i}}=0(\mathrm{i}=1, \ldots, 10) \\
\mathrm{u}_{1} \mathrm{u}_{6}=-\mathrm{u}_{2} \mathrm{u}_{3}=\mathrm{v}_{4} ; \mathrm{u}_{1} \mathrm{u}_{7}=-\mathrm{u}_{2} \mathrm{u}_{4}=\mathrm{v}_{5} ; \mathrm{u}_{1} \mathrm{u}_{8}=-\mathrm{u}_{2} \mathrm{u}_{5}=\mathrm{v}_{6} \\
\mathrm{u}_{1} \mathrm{u}_{12}=-\mathrm{u}_{2} \mathrm{u}_{9}=\mathrm{u}_{3} \mathrm{u}_{7}=-\mathrm{u}_{4} \mathrm{u}_{6}=\mathrm{v}_{7} \\
\mathrm{u}_{1} \mathrm{u}_{13}=-\mathrm{u}_{2} \mathrm{u}_{10}=\mathrm{u}_{3} \mathrm{u}_{8}=-\mathrm{u}_{5} \mathrm{u}_{6}=\mathrm{v}_{8} \\
\mathrm{u}_{1} \mathrm{u}_{14}=-\mathrm{u}_{2} \mathrm{u}_{11}=\mathrm{u}_{4} \mathrm{u}_{8}=-\mathrm{u}_{5} \mathrm{u}_{7}=-\mathrm{v}_{9} \\
\mathrm{u}_{1} \mathrm{u}_{16}=\mathrm{u}_{3} \mathrm{u}_{14}=\mathrm{u}_{5} \mathrm{u}_{12}=\mathrm{u}_{6} \mathrm{u}_{11}=\mathrm{u}_{8} \mathrm{u}_{9}=\mathrm{v}_{10} \\
\mathrm{u}_{2} \mathrm{u}_{15}=\mathrm{u}_{4} \mathrm{u}_{13}=\mathrm{u}_{7} \mathrm{u}_{10}=-\mathrm{v}_{10} \\
\mathrm{u}_{1} \mathrm{v}_{1}=\mathrm{u}_{3} ; \mathrm{u}_{1} \mathrm{v}_{2}=\mathrm{u}_{4} ; \mathrm{u}_{1} \mathrm{v}_{3}=\mathrm{u}_{5} ; \mathrm{u}_{2} \mathrm{v}_{1}=\mathrm{u}_{6} ; \mathrm{u}_{2} \mathrm{v}_{2}=\mathrm{u}_{7} ; \mathrm{u}_{2} \mathrm{v}_{3}=\mathrm{u}_{8} \\
\mathrm{u}_{3} \mathrm{v}_{2}=-\mathrm{u}_{4} \mathrm{v}_{1}=\mathrm{u}_{9} ; \mathrm{u}_{3} \mathrm{v}_{3}=-\mathrm{u}_{5} \mathrm{v}_{1}=\mathrm{u}_{10} ; \mathrm{u}_{4} \mathrm{v}_{3}=-\mathrm{u}_{5} \mathrm{v}_{2}=\mathrm{u}_{11} \\
\mathrm{u}_{6} \mathrm{v}_{2}=-\mathrm{u}_{7} \mathrm{v}_{1}=\mathrm{u}_{12} ; \mathrm{u}_{6} \mathrm{v}_{3}=-\mathrm{u}_{8} \mathrm{v}_{1}=\mathrm{u}_{13} ; \mathrm{u}_{7} \mathrm{v}_{3}=-\mathrm{u}_{8} \mathrm{v}_{2}=\mathrm{u}_{14} \\
\mathrm{u}_{9} \mathrm{v}_{3}=-\mathrm{u}_{10} \mathrm{v}_{2}=\mathrm{u}_{11} \mathrm{v}_{1}=\mathrm{u}_{15} ; \mathrm{u}_{12} \mathrm{v}_{3}=-\mathrm{u}_{13} \mathrm{v}_{2}=\mathrm{u}_{14} \mathrm{v}_{1}=-\mathrm{u}_{16} \\
\text { os outros produtos são nulos }
\end{array}\right.
$$

É fácil ver que os P-monômios $\mathrm{U}, \mathrm{UV}=\mathrm{Fu}_{3} \oplus \ldots \oplus \mathrm{Fu}_{16},(\mathrm{UV}) \mathrm{V}=$ $=\mathrm{Fu}_{9} \oplus \ldots \oplus \mathrm{Fu}_{16}$ e $[(\mathrm{UV}) \mathrm{V}] \mathrm{V}=\mathrm{Fu}_{15} \oplus \mathrm{Fu}_{16}$ não são ideais de $\mathrm{A}$, pois $\mathrm{u}_{1} \mathrm{u}_{16}=\mathrm{v}_{10}$. O P-monômio $\mathrm{V}$ não é ideal de $\mathrm{A}$ pois $\mathrm{u}_{1} \mathrm{v}_{1}=\mathrm{u}_{3} \notin \mathrm{V}$. Além disso, os $\mathrm{P}$-monômios $\mathrm{U}^{2}=\mathrm{U}(\mathrm{UV})=\mathrm{Fv}_{4} \oplus \ldots \oplus \mathrm{Fv}_{10}, \mathrm{U}[(\mathrm{UV}) \mathrm{V}]=$ $=\mathrm{Fv}_{7} \oplus \ldots \oplus \mathrm{Fv}_{10}, \mathrm{U}^{3}=\mathrm{U}^{4}=\mathrm{U}^{3} \mathrm{~V}=\mathrm{U}[\mathrm{U}(\mathrm{UV})]=0$ são ideais de $\mathrm{A}$. Isto mostra que os seguintes $\mathrm{P}$-subespaços do Grupo II não são ideais de A:

7. (UV)V

12. $[(\mathrm{UV}) \mathrm{V}] \mathrm{V}$

18. $\mathrm{U}^{3} \oplus \mathrm{V}=\mathrm{V}$

30. $\mathrm{UV} \oplus \mathrm{U}^{4}=\mathrm{UV}$

42. $(\mathrm{UV}) \mathrm{V}+\mathrm{U}[\mathrm{U}(\mathrm{UV})]=(\mathrm{UV}) \mathrm{V}$

49. $\mathrm{U}[\mathrm{U}(\mathrm{UV})]+[(\mathrm{UV}) \mathrm{V}] \mathrm{V}=[(\mathrm{UV}) \mathrm{V}] \mathrm{V}$

52. $\mathrm{U}^{3} \mathrm{~V}+[(\mathrm{UV}) \mathrm{V}] \mathrm{V}=[(\mathrm{UV}) \mathrm{V}] \mathrm{V}$

54. $\left[\mathrm{U}^{3}+(\mathrm{UV}) \mathrm{V}\right] \ominus \mathrm{V}=(\mathrm{UV}) \mathrm{V} \ominus \mathrm{V}\left(\right.$ pois $\left.\mathrm{u}_{1} \mathrm{v}_{1} \notin(\mathrm{UV}) \mathrm{V}\right)$

55. $\left\{\mathrm{U}^{3}+[(\mathrm{UV}) \mathrm{V}] \mathrm{V}\right\} \oplus \mathrm{V}=[(\mathrm{UV}) \mathrm{V}] \mathrm{V} \oplus \mathrm{V}\left(\right.$ pois $\left.\mathrm{u}_{1} \mathrm{v}_{1} \notin[(\mathrm{UV}) \mathrm{V}] \mathrm{V}\right)$ 
64. $\quad \mathrm{UV} \oplus\left\{\mathrm{U}^{4}+\mathrm{U}[(\mathrm{UV}) \mathrm{V}]\right\}=\mathrm{UV} \oplus \mathrm{U}[(\mathrm{UV}) \mathrm{V}]\left(\right.$ pois $\left._{2} \mathrm{u}_{4} \notin \mathrm{U}[(\mathrm{UV}) \mathrm{V}]\right)$ 67. $\left[\mathrm{U}^{3}+(\mathrm{UV}) \mathrm{V}\right] \oplus \mathrm{U}^{4}=(\mathrm{UV}) \mathrm{V}$

70. $\left\{\mathrm{U}^{3}+[(\mathrm{UV}) \mathrm{V}] \mathrm{V}\right\} \oplus \mathrm{U}^{4}=[(\mathrm{UV}) \mathrm{V}] \mathrm{V}$

75. $\{(\mathrm{UV}) \mathrm{V}+\mathrm{U}[\mathrm{U}(\mathrm{UV})]\} \oplus \mathrm{U}^{4}=(\mathrm{UV}) \mathrm{V}$

79. $\{\mathrm{U}[\mathrm{U}(\mathrm{UV})]+[(\mathrm{UV}) \mathrm{V}] \mathrm{V}\} \oplus \mathrm{U}^{4}=[(\mathrm{UV}) \mathrm{V}] \mathrm{V}$

Observação: Nos exemplos 27 a 30 obtivemos uma lista de P-subespaços do Grupo II que não são ideais de A. Se compararmos com a lista de Psubespaços que sabemos que efetivamente pertencem ao Grupo II, veremos que tais listas coincidem.

Daremos agora um exemplo de um ideal que não é polinomial.

Exemplo 31: Consideremos A a t-álgebra de posto 3 e dimensão 5 com base $\left\{\mathrm{e}, \mathrm{u}_{1}, \mathrm{u}_{2}, \mathrm{u}_{1}^{2}, \mathrm{v}\right\}$ e tábua de multiplicação:

$$
\left\{\begin{array}{l}
\mathrm{e}^{2}=\mathrm{e} ; 2 \mathrm{eu}_{1}=\mathrm{u}_{1} ; 2 \mathrm{eu}_{2}=\mathrm{u}_{2} \\
\mathrm{u}_{\mathrm{i}} \mathrm{u}_{\mathrm{j}}=\mathrm{u}_{1}^{2} \\
\text { os outros produtos são nulos }
\end{array}\right.
$$

$\mathrm{O}$ ideal $\mathrm{L}=\{\mathrm{u} \in \mathrm{U}: \mathrm{uU}=0\}$ não é polinomial. De fato, temos que $\mathrm{U}$ é gerado por $\left\{\mathrm{u}_{1}, \mathrm{u}_{2}\right\}, \mathrm{V}$ é gerado por $\left\{\mathrm{u}_{1}^{2}, \mathrm{v}\right\}, \mathrm{U}^{2}=\mathrm{Fu}_{1}{ }^{2}$ e todos os outros P-subespaços (como, por exemplo, UV, $\mathrm{U}^{3}, \mathrm{U}(\mathrm{UV}),(\mathrm{UV}) \mathrm{V}$, $\left.\mathrm{U}^{4}, \mathrm{U}[\mathrm{U}(\mathrm{UV})], \mathrm{U}[(\mathrm{UV}) \mathrm{V}], \mathrm{U}^{3} \mathrm{~V},[(\mathrm{UV}) \mathrm{V}] \mathrm{V}\right)$ são nulos. Dado um elemento arbitrário de $\mathrm{U}$, digamos $\mathrm{au}_{1}+\mathrm{bu}_{2} \in \mathrm{U}$, a,b $\in \mathrm{F}$, temos que $\left(\mathrm{u}_{1}-\mathrm{u}_{2}\right)\left(\mathrm{au}_{1}+\mathrm{bu}_{2}\right)=\mathrm{au}_{1}^{2}+\mathrm{bu}_{1}^{2}-\mathrm{au}_{1}^{2}-\mathrm{bu}_{1}^{2}=0$ e, portanto, $0 \neq$ $\neq \mathrm{u}_{1}-\mathrm{u}_{2} \in \mathrm{L}$, o que mostra que $\operatorname{dim} \mathrm{L}>0$. Além disso, como $\mathrm{u}_{1}^{2} \neq 0$ por ser um elemento da base de $\mathrm{A}$, segue que $\mathrm{u}_{1} \notin \mathrm{L}$ e portanto $\operatorname{dim} \mathrm{L}<$ $<2$. Logo, $\operatorname{dim} \mathrm{L}=1$ donde concluimos que $\mathrm{L}=\mathrm{F}\left(\mathrm{u}_{1}-\mathrm{u}_{2}\right)$ e que portanto L não é polinomial. 
Observação: O Exemplo 31 acima serve também como resposta negativa a um dos desdobramentos do Problema 6 colocado no Capítulo 1. 


\section{Capítulo 7}

\section{Sobre a invariância relativa dos P-subespaços}

Neste capítulo, introduziremos a noção de "invariância relativa" de um P-subespaço. Para um caso particular, estudaremos a migração de Psubespaços do Grupo II para o Grupo I.

\subsection{Invariância relativa: definição}

Fixemos $\mathrm{A}=\mathrm{Fe} \oplus \mathrm{U} \oplus \mathrm{V}$ uma t-álgebra de posto 3 sobre o corpo $\mathrm{F}$ e $\mathrm{S} \subseteq \mathrm{U}$ um subconjunto arbitrário do P-monômio U, contendo a origem. Os cálculos que fizemos no Capítulo 5 correspondem a tomar $\mathrm{S}=\mathrm{U}$ na definição que segue.

Definição: Dizemos que um $\mathrm{P}$-subespaço $\mathrm{p}(\mathrm{U}, \mathrm{V})$ é S-invariante se $\mathrm{p}(\mathrm{U}, \mathrm{V})$ é um P-subespaço invariante por mudança de idempotentes quando fazemos $u_{0}$ variar em $\mathrm{S}$; em outras palavras, $\mathrm{p}(\mathrm{U}, \mathrm{V})$ é S-invariante se e somente se $p(U, V)=p\left(U_{0}, V_{0}\right)$ para todo $u_{0} \in S$.

Observação 1: É claro que todo P-subespaço invariante é também Sinvariante, para todo $\mathrm{S} \subseteq \mathrm{U}$. 
Observação 2: Se $u_{0} \in \operatorname{ann}_{\mathrm{U}} \mathrm{N}=\{u \in U: u N=0\}$, então todos os P-subespaços do Grupo II migram para o Grupo I.

\subsection{Um caso particular do Problema 8}

No capítulo 1, colocamos a seguinte questão: fixado um subconjunto $S$ de $\mathrm{U}$, com $0 \in \mathrm{S}$, quais são os $\mathrm{P}$-subespaços $\mathrm{p}(\mathrm{U}, \mathrm{V})$ tais que $\mathrm{p}(\mathrm{U}, \mathrm{V})=$ $=\mathrm{p}\left(\mathrm{U}_{0}, \mathrm{~V}_{0}\right)$ para todo $\mathrm{u}_{0} \in \mathrm{S}$ ? Nesta seção, estudamos o caso em que $\mathrm{S}=\mathrm{Fu}_{0} \mathrm{v}_{0}$, sendo $\mathrm{u}_{0} \in \mathrm{U}$ e $\mathrm{v}_{0} \in \mathrm{V}$; isto é, $\mathrm{S}$ é uma reta de $\mathrm{U}$, passando pela origem e na direção do vetor $\mathrm{u}_{0} \mathrm{v}_{0}$. Lembrando que $\left(\mathrm{u}_{0} \mathrm{v}_{0}\right)^{2}=0$ (por (1.17)), consideremos um idempotente $\mathrm{e}_{0}=\mathrm{e}+\mathrm{u}_{0} \mathrm{v}_{0}$; isto é, fixamos $\alpha=1$ (pois isto facilita os cálculos sem afetar os resultados). A partir de (1.20), (1.21) e das Proposições 1 a 10 do Capítulo 5, obtemos as seguintes inclusões para os $\mathrm{P}$-monômios de graus 1 até 4 :

$$
\begin{gathered}
\mathrm{U}_{0} \subseteq \mathrm{U} \oplus \mathrm{U}(\mathrm{UV}) \\
\mathrm{V}_{0} \subseteq(\mathrm{UV}) \mathrm{V} \oplus \mathrm{V} \\
\mathrm{U}_{0} \mathrm{~V}_{0} \subseteq \mathrm{UV} \oplus(\mathrm{UV})^{2} \\
\mathrm{U}_{0}\left(\mathrm{U}_{0} \mathrm{~V}_{0}\right) \subseteq(\mathrm{UV})[\mathrm{U}(\mathrm{UV})] \oplus \mathrm{U}(\mathrm{UV}) \\
\left(\mathrm{U}_{0} \mathrm{~V}_{0}\right) \mathrm{V}_{0} \subseteq(\mathrm{UV}) \mathrm{V} \oplus(\mathrm{UV})[(\mathrm{UV}) \mathrm{V}] \\
\mathrm{U}_{0}^{2} \subseteq(\mathrm{UV}) \mathrm{U}^{2} \oplus \mathrm{U}^{2} \\
\mathrm{U}_{0}^{3} \subseteq \mathrm{U}^{3} \oplus(\mathrm{UV}) \mathrm{U}^{3} \\
\mathrm{U}_{0}^{4} \subseteq(\mathrm{UV}) \mathrm{U}^{4} \oplus \mathrm{U}^{4} \\
\mathrm{U}_{0}^{3} \mathrm{~V}_{0} \subseteq \mathrm{U}^{3} \mathrm{~V} \oplus(\mathrm{UV})\left(\mathrm{U}^{3} \mathrm{~V}\right) \\
\mathrm{U}_{0}\left[\mathrm{U}_{0}\left(\mathrm{U}_{0} \mathrm{~V}_{0}\right)\right] \subseteq \mathrm{U}[\mathrm{U}(\mathrm{UV})] \oplus(\mathrm{UV})\{\mathrm{U}[\mathrm{U}(\mathrm{UV})]\} \\
\mathrm{U}_{0}\left[\left(\mathrm{U}_{0} \mathrm{~V}_{0}\right) \mathrm{V}_{0}\right] \subseteq(\mathrm{UV})\{\mathrm{U}[(\mathrm{UV}) \mathrm{V}]\} \oplus \mathrm{U}[(\mathrm{UV}) \mathrm{V}] \\
{\left[\left(\mathrm{U}_{0} \mathrm{~V}_{0}\right) \mathrm{V}_{0}\right] \mathrm{V}_{0} \subseteq[(\mathrm{UV}) \mathrm{V}] \mathrm{V} \oplus(\mathrm{UV})\{[(\mathrm{UV}) \mathrm{V}] \mathrm{V}\}}
\end{gathered}
$$


Lema 7.1 Nas condições acima descritas, os seguintes P-subespaços do Grupo II sâo S-invariantes:

14. $U \oplus U(U V)$

19. $(U V) V \oplus V$

25. $(U V) V \oplus U^{2}$

26. $U[U(U V)] \oplus U^{2}$

27. $U^{3} V \oplus U^{2}$

31. $U V \oplus U[(U V) V]$

35. $U^{3} \oplus U[(U V) V]$

37. $(U V) V \oplus U(U V)$

39. $U^{3} V \oplus U(U V)$

46. $U^{3} V \oplus U^{4}$

48. $U[U(U V)] \oplus U[(U V) V]$

54. $\left[U^{3}+(U V) V\right] \oplus V$

56. $\{(U V) V+U[U(U V)]\} \oplus V$

61. $\{(U V) V+U[U(U V)]\} \oplus U^{2}$

62. $\{U[U(U V)]+[(U V) V] V\} \oplus U^{2}$

63. $\left\{U^{3} V+[(U V) V] V\right\} \oplus U^{2}$

64. $U V \oplus\left\{U^{4}+U[(U V) V]\right\}$

68. $\left[U^{3}+(U V) V\right] \oplus U[(U V) V]$

71. $\left\{U^{3}+[(U V) V] V\right\} \oplus U[(U V) V]$

74. $\left\{U^{3} V+[(U V) V] V\right\} \oplus U(U V)$

76. $(U V) V \oplus\left\{U^{4}+U[(U V) V]\right\}$

77. $\{(U V) V+U[U(U V)]\} \oplus U[(U V) V]$

80. $U^{3} V \oplus\left\{U^{4}+U[(U V) V]\right\}$

83. $\{U[U(U V)]+[(U V) V] V\} \oplus U[(U V) V]$

89. $\left\{U^{3} V+[(U V) V] V\right\} \oplus\left\{U^{4}+U[(U V) V]\right\}$

Prova: Como já vimos no Capítulo 5, basta mostrarmos, em cada caso. que $\mathrm{p}\left(\mathrm{U}_{0}, \mathrm{~V}_{0}\right) \subseteq \mathrm{p}(\mathrm{U}, \mathrm{V})$, pois a inclusão contrária é obtida por simetria. Cada caso será identificado por seu correspondente número acima listado.

14. De (7.1) e (7.4), respectivamente, temos que $\mathrm{U}_{0} \subseteq \mathrm{U} \oplus \mathrm{U}(\mathrm{UV})$ e 
$\mathrm{U}_{0}\left(\mathrm{U}_{0} \mathrm{~V}_{0}\right) \subseteq(\mathrm{UV})[\mathrm{U}(\mathrm{UV})] \oplus \mathrm{U}(\mathrm{UV}) \subseteq \mathrm{U} \oplus \mathrm{U}(\mathrm{UV})$. Portanto, $\mathrm{U}_{0} \oplus$ $\oplus \mathrm{U}_{0}\left(\mathrm{U}_{0} \mathrm{~V}_{0}\right) \subseteq \mathrm{U} \oplus \mathrm{U}(\mathrm{UV})$.

19. A inclusão $\left(\mathrm{U}_{0} \mathrm{~V}_{0}\right) \mathrm{V}_{0} \subseteq(\mathrm{UV}) \mathrm{V} \oplus \mathrm{V}$ segue de (7.5). Além disso, $\mathrm{V}_{0} \subseteq$ $\subseteq(\mathrm{UV}) \mathrm{V} \oplus \mathrm{V}($ por $(7.2))$ e assim $\left(\mathrm{U}_{0} \mathrm{~V}_{0}\right) \mathrm{V}_{0} \oplus \mathrm{V}_{0} \subseteq(\mathrm{UV}) \mathrm{V} \oplus \mathrm{V}$.

25. Novamente de (7.5), obtemos $\left(\mathrm{U}_{0} \mathrm{~V}_{0}\right) \mathrm{V}_{0} \subseteq(\mathrm{UV}) \mathrm{V} \oplus \mathrm{U}^{2}$ e de (7.6) segue que $\mathrm{U}_{0}^{2} \subseteq(\mathrm{UV}) \mathrm{V} \oplus \mathrm{U}^{2}$, pois $\mathrm{U}^{2} \subseteq \mathrm{V}$. Logo, $\left(\mathrm{U}_{0} \mathrm{~V}_{0}\right) \mathrm{V}_{0} \oplus \mathrm{U}_{0}^{2} \subseteq$ $\subseteq(\mathrm{UV}) \mathrm{V} \oplus \mathrm{U}^{2}$.

26. De (7.10), segue que $\mathrm{U}_{0}\left[\mathrm{U}_{0}\left(\mathrm{U}_{0} \mathrm{~V}_{0}\right)\right] \subseteq \mathrm{U}[\mathrm{U}(\mathrm{UV})] \oplus \mathrm{U}^{2}$ e de (7.6) obtemos $\mathrm{U}_{0}^{2} \subseteq(\mathrm{UV}) \mathrm{U}^{2} \oplus \mathrm{U}^{2} \subseteq \mathrm{U}[\mathrm{U}(\mathrm{UV})] \oplus \mathrm{U}^{2}$, sendo esta última inclusão dada no diagrama da página 9. Dessa forma, $\mathrm{U}_{0}\left[\mathrm{U}_{0}\left(\mathrm{U}_{0} \mathrm{~V}_{0}\right)\right] \oplus \mathrm{U}_{0}^{2} \subseteq$ $\subseteq \mathrm{U}[\mathrm{U}(\mathrm{UV})] \oplus \mathrm{U}^{2}$.

27. A inclusão $\mathrm{U}_{0}^{3} \mathrm{~V}_{0} \subseteq \mathrm{U}^{3} \mathrm{~V} \oplus \mathrm{U}^{2}$ é consequência de (7.9). Por outro lado, de (7.6) segue que $\mathrm{U}_{0}^{2} \subseteq(\mathrm{UV}) \mathrm{U}^{2} \oplus \mathrm{U}^{2}=\mathrm{U}^{3} \mathrm{~V} \oplus \mathrm{U}^{2}$, pelo Lema 3.1. Logo, $\mathrm{U}_{0}^{3} \mathrm{~V}_{0} \oplus \mathrm{U}_{0}^{2} \subseteq \mathrm{U}^{3} \mathrm{~V} \oplus \mathrm{U}^{2}$.

31. De (7.3) e do Lema 3.1 obtemos $\mathrm{U}_{0} \mathrm{~V}_{0} \subseteq \mathrm{UV} \oplus(\mathrm{UV})^{2}=\mathrm{UV} \oplus$ $\oplus \mathrm{U}[(\mathrm{UV}) \mathrm{V}]$. Além disso, a inclusão $\mathrm{U}_{0}\left[\left(\mathrm{U}_{0} \mathrm{~V}_{0}\right) \mathrm{V}_{0}\right] \subseteq(\mathrm{UV})\{\mathrm{U}[(\mathrm{UV}) \mathrm{V}]\} \oplus$ $\oplus \mathrm{U}[(\mathrm{UV}) \mathrm{V}] \subseteq \mathrm{UU}^{2} \oplus \mathrm{U}[(\mathrm{UV}) \mathrm{V}] \subseteq \mathrm{UV} \oplus \mathrm{U}[(\mathrm{UV}) \mathrm{V}]$ segue de $(7.11)$ e de $\mathrm{U}[(\mathrm{UV}) \mathrm{V}] \subseteq \mathrm{U}^{2} \subseteq \mathrm{V}$. Portanto, $\mathrm{U}_{0} \mathrm{~V}_{0} \oplus \mathrm{U}_{0}\left[\left(\mathrm{U}_{0} \mathrm{~V}_{0}\right) \mathrm{V}_{0}\right] \subseteq \mathrm{UV} \oplus \mathrm{U}[(\mathrm{UV}) \mathrm{V}]$.

35. Como $\mathrm{U}_{0}^{3} \subseteq \mathrm{U}^{3} \oplus(\mathrm{UV}) \mathrm{U}^{3}$ (por (7.7)) e além disso $\mathrm{U}^{3} \subseteq \mathrm{UV}$, segue que $(\mathrm{UV}) \mathrm{U}^{3} \subseteq(\mathrm{UV})^{2}=\mathrm{U}[(\mathrm{UV}) \mathrm{V}]$ e portanto $\mathrm{U}_{0}^{3} \subseteq \mathrm{U}^{3} \oplus \mathrm{U}[(\mathrm{UV}) \mathrm{V}]$. Por outro lado, de (7.11) obtemos $\mathrm{U}_{0}\left[\left(\mathrm{U}_{0} \mathrm{~V}_{0}\right) \mathrm{V}_{0}\right] \subseteq(\mathrm{UV}) \mathrm{U}^{2} \oplus \mathrm{U}[(\mathrm{UV}) \mathrm{V}] \subseteq$ $\subseteq \mathrm{U}^{3} \oplus \mathrm{U}[(\mathrm{UV}) \mathrm{V}]$. Logo, $\mathrm{U}_{0}^{3} \oplus \mathrm{U}_{0}\left[\left(\mathrm{U}_{0} \mathrm{~V}_{0}\right) \mathrm{V}_{0}\right] \subseteq \mathrm{U}^{3} \oplus \mathrm{U}[(\mathrm{UV}) \mathrm{V}]$.

37. A inclusão $\left(\mathrm{U}_{0} \mathrm{~V}_{0}\right) \mathrm{V}_{0} \oplus \mathrm{U}_{0}\left(\mathrm{U}_{0} \mathrm{~V}_{0}\right) \subseteq(\mathrm{UV}) \mathrm{V} \oplus \mathrm{U}(\mathrm{UV})$ segue de (7.4), (7.5) e de $\mathrm{U}(\mathrm{UV}) \subseteq \mathrm{V}$ e $\mathrm{UV} \subseteq \mathrm{U}$.

39. De (7.9) e $\mathrm{U}^{3} \subseteq \mathrm{UV} \subseteq \mathrm{U}$ temos que $\mathrm{U}_{0}^{3} \mathrm{~V}_{0} \subseteq \mathrm{U}^{3} \mathrm{~V} \oplus \mathrm{U}(\mathrm{UV})$. Por outro lado, (UV) $[\mathrm{U}(\mathrm{UV})] \subseteq(\mathrm{UV}) \mathrm{U}^{2}=\mathrm{U}^{3} \mathrm{~V}$ e portanto, de (7.4), segue que $\mathrm{U}_{0}\left(\mathrm{U}_{0} \mathrm{~V}_{0}\right) \subseteq \mathrm{U}^{3} \mathrm{~V} \ominus \mathrm{U}(\mathrm{UV})$. Logo, $\mathrm{U}_{0}^{3} \mathrm{~V}_{0} \ominus \mathrm{U}_{0}\left(\mathrm{U}_{0} \mathrm{~V}_{0}\right) \subseteq \mathrm{U}^{3} \mathrm{~V} \oplus \mathrm{U}(\mathrm{UV})$. 
46. Da identidade de Jacobi é fácil ver que (UV) $U^{4}=U^{5} \mathrm{~V}$ e como $\mathrm{U}^{5} \subseteq \mathrm{U}^{3}$, segue que (UV) $\mathrm{U}^{4} \subseteq \mathrm{U}^{3} \mathrm{~V}$. Assim, de (7.8) e (7.9) temos que $\mathrm{U}_{0}^{3} \mathrm{~V}_{0} \oplus \mathrm{U}_{0}^{4} \subseteq\left[\mathrm{U}^{3} \mathrm{~V} \oplus(\mathrm{UV})\left(\mathrm{U}^{3} \mathrm{~V}\right)\right]+\left[\mathrm{U}^{3} \mathrm{~V} \oplus \mathrm{U}^{4}\right] \subseteq \mathrm{U}^{3} \mathrm{~V} \oplus \mathrm{U}^{4}$, sendo esta última inclusão consequência de $(\mathrm{UV})\left(\mathrm{U}^{3} \mathrm{~V}\right) \subseteq \mathrm{UU}^{3}=\mathrm{U}^{4}$.

48. De (7.10) e de $\mathrm{U}[\mathrm{U}(\mathrm{UV})] \subseteq \mathrm{U}^{3} \subseteq \mathrm{UV}$ segue que $\mathrm{U}_{0}\left[\mathrm{U}_{0}\left(\mathrm{U}_{0} \mathrm{~V}_{0}\right)\right] \subseteq$ $\subseteq \mathrm{U}[\mathrm{U}(\mathrm{UV})] \oplus(\mathrm{UV}) \mathrm{U}^{3} \subseteq \mathrm{U}[\mathrm{U}(\mathrm{UV})] \oplus(\mathrm{UV})^{2}=\mathrm{U}[\mathrm{U}(\mathrm{UV})] \oplus \mathrm{U}[(\mathrm{UV}) \mathrm{V}]$, uma vez que $(U V)^{2}=\mathrm{U}[(\mathrm{UV}) \mathrm{V}]$. Por outro lado, de (7.11) e de $U V \subseteq$ $\subseteq \mathrm{U}$, segue que $\mathrm{U}_{0}\left[\left(\mathrm{U}_{0} \mathrm{~V}_{0}\right) \mathrm{V}_{0}\right] \subseteq \mathrm{U}[\mathrm{U}(\mathrm{UV})] \oplus \mathrm{U}[(\mathrm{UV}) \mathrm{V}]$. Dessa forma, $\mathrm{U}_{0}\left[\mathrm{U}_{0}\left(\mathrm{U}_{0} \mathrm{~V}_{0}\right)\right] \oplus \mathrm{U}_{0}\left[\left(\mathrm{U}_{0} \mathrm{~V}_{0}\right) \mathrm{V}_{0}\right] \subseteq \mathrm{U}[\mathrm{U}(\mathrm{UV})] \oplus \mathrm{U}[(\mathrm{UV}) \mathrm{V}]$.

54. A inclusão $\left[\mathrm{U}_{0}^{3}+\left(\mathrm{U}_{0} \mathrm{~V}_{0}\right) \mathrm{V}_{0}\right] \oplus \mathrm{V}_{0} \subseteq\left[\mathrm{U}^{3}+(\mathrm{UV}) \mathrm{V}\right] \oplus \mathrm{V}$ segue de (7.2), (7.5), (7.7) e de (UV) $[(\mathrm{UV}) \mathrm{V}] \subseteq \mathrm{U}^{2} \subseteq \mathrm{V},(\mathrm{UV}) \mathrm{U}^{3} \subseteq \mathrm{U}^{4} \subseteq \mathrm{V}$.

Nos próximas demontrações, usaremos os casos provados acima. Faremos referência a eles usando o seu número (entre parêntesis).

56. Em (19) mostramos que $\left(\mathrm{U}_{0} \mathrm{~V}_{0}\right) \mathrm{V}_{0} \oplus \mathrm{V}_{0} \subseteq(\mathrm{UV}) \mathrm{V} \oplus \mathrm{V}$. Resta portanto analisarmos apenas $\mathrm{U}_{0}\left[\mathrm{U}_{0}\left(\mathrm{U}_{0} \mathrm{~V}_{0}\right)\right]$. Mas de (7.10) segue que $\mathrm{U}_{0}\left[\mathrm{U}_{0}\left(\mathrm{U}_{0} \mathrm{~V}_{0}\right)\right] \subseteq \mathrm{U}[\mathrm{U}(\mathrm{UV})] \oplus \mathrm{V}$ e portanto $\left\{\left(\mathrm{U}_{0} \mathrm{~V}_{0}\right) \mathrm{V}_{0}+\mathrm{U}_{0}\left[\mathrm{U}_{0}\left(\mathrm{U}_{0} \mathrm{~V}_{0}\right)\right]\right\} \oplus$ $\oplus \mathrm{V}_{0} \subseteq\{(\mathrm{UV}) \mathrm{V}+\mathrm{U}[\mathrm{U}(\mathrm{UV})]\} \oplus \mathrm{V}$.

61. Basta usarmos (25) e (26).

62. Novamente usando (26), resta apenas estudarmos o P-monômio $\left[\left(\mathrm{U}_{0} \mathrm{~V}_{0}\right) \mathrm{V}_{0}\right] \mathrm{V}_{0}$. Mas de (7.12), temos que $\left[\left(\mathrm{U}_{0} \mathrm{~V}_{0}\right) \mathrm{V}_{0}\right] \mathrm{V}_{0} \subseteq[(\mathrm{UV}) \mathrm{V}] \mathrm{V} \odot$ $\oplus \mathrm{U}^{2}$, e portanto $\left.\left\{\mathrm{U}_{0}\left[\mathrm{U}_{0}\left(\mathrm{U}_{0} \mathrm{~V}_{0}\right)\right]+\left(\mathrm{U}_{0} \mathrm{~V}_{0}\right) \mathrm{V}_{0}\right] \mathrm{V}_{0}\right\} \oplus \mathrm{U}_{0}^{2} \subseteq\{\mathrm{U}[\mathrm{U}(\mathrm{UV})]+$ $+[(\mathrm{UV}) \mathrm{V}] \mathrm{V}\} \oplus \mathrm{U}^{2}$.

63. Já vimos em (27) que $\mathrm{U}_{0}^{3} \mathrm{~V}_{0} \oplus \mathrm{U}_{0}^{2} \subseteq \mathrm{U}^{3} \mathrm{~V} \oplus \mathrm{U}^{2}$. Em (62) ficou mostrado que $\left[\left(\mathrm{U}_{0} \mathrm{~V}_{0}\right) \mathrm{V}_{0}\right] \mathrm{V}_{0} \subseteq[(\mathrm{UV}) \mathrm{V}] \mathrm{V} \oplus \mathrm{U}^{2}$ e com isto obtemos a inclusão $\left\{\mathrm{U}_{0}^{3} \mathrm{~V}_{0}+\left[\left(\mathrm{U}_{0} \mathrm{~V}_{0}\right) \mathrm{V}_{0}\right] \mathrm{V}_{0}\right\} \oplus \mathrm{U}_{0}^{2} \subseteq\left\{\mathrm{U}^{3} \mathrm{~V}+[(\mathrm{UV}) \mathrm{V}] \mathrm{V}\right\} \oplus \mathrm{U}^{2}$.

64. Usando (31), resta apenas analisarmos o $\mathrm{P}$-monômio $\mathrm{U}_{0}^{4}$. De (7.8), segue que $\mathrm{U}_{0}^{+} \subseteq \mathrm{UV} \ominus \mathrm{U}^{4}$ (pois $\mathrm{UV} \subseteq \mathrm{U}$ e $\mathrm{U}^{4} \subseteq \mathrm{V}$ ) e portanto $\mathrm{U}_{0} \mathrm{~V}_{0} \odot$ 
$\oplus\left\{\mathrm{U}_{0}^{4}+\mathrm{U}_{0}\left[\left(\mathrm{U}_{0} \mathrm{~V}_{0}\right) \mathrm{V}_{0}\right]\right\} \subseteq \mathrm{UV} \oplus\left\{\mathrm{U}^{4}+\mathrm{U}[(\mathrm{UV}) \mathrm{V}]\right\}$.

68. De (7.5) segue que $\left(\mathrm{U}_{0} \mathrm{~V}_{0}\right) \mathrm{V}_{0} \subseteq(\mathrm{UV}) \mathrm{V} \oplus(\mathrm{UV})^{2}=(\mathrm{UV}) \mathrm{V} \oplus$ $\oplus \mathrm{U}[(\mathrm{UV}) \mathrm{V}]$. Logo, de $(35)$, temos $\left[\mathrm{U}_{0}^{3}+\left(\mathrm{U}_{0} \mathrm{~V}_{0}\right) \mathrm{V}_{0}\right] \oplus \mathrm{U}_{0}\left[\left(\mathrm{U}_{0} \mathrm{~V}_{0}\right) \mathrm{V}_{0}\right] \subseteq$ $\subseteq\left[\mathrm{U}^{3}+(\mathrm{UV}) \mathrm{V}\right] \oplus \mathrm{U}[(\mathrm{UV}) \mathrm{V}]$.

71. Como $\left[\left(\mathrm{U}_{0} \mathrm{~V}_{0}\right) \mathrm{V}_{0}\right] \mathrm{V}_{0} \subseteq[(\mathrm{UV}) \mathrm{V}] \mathrm{V} \oplus(\mathrm{UV})^{2}=[(\mathrm{UV}) \mathrm{V}] \mathrm{V} \oplus \mathrm{U}[(\mathrm{UV}) \mathrm{V}]$ (por (7.12)), de (35) obtemos $\left\{\mathrm{U}_{0}^{3}+\left[\left(\mathrm{U}_{0} \mathrm{~V}_{0}\right) \mathrm{V}_{0}\right] \mathrm{V}_{0}\right\} \oplus \mathrm{U}_{0}\left[\left(\mathrm{U}_{0} \mathrm{~V}_{0}\right) \mathrm{V}_{0}\right] \subseteq$ $\subseteq\left\{\mathrm{U}^{3}+[(\mathrm{UV}) \mathrm{V}] \mathrm{V}\right\} \oplus \mathrm{U}[(\mathrm{UV}) \mathrm{V}]$.

74. Já vimos, em (39), que $\mathrm{U}_{0}^{3} \mathrm{~V}_{0} \oplus \mathrm{U}_{0}\left(\mathrm{U}_{0} \mathrm{~V}_{0}\right) \subseteq \mathrm{U}^{3} \mathrm{~V} \oplus \mathrm{U}(\mathrm{UV})$. Por outro lado, de (7.12), $\left[\left(\mathrm{U}_{0} \mathrm{~V}_{0}\right) \mathrm{V}_{0}\right] \mathrm{U}_{0} \subseteq[(\mathrm{UV}) \mathrm{V}] \mathrm{V} \oplus \mathrm{U}(\mathrm{UV})$, uma vez que $(\mathrm{UV}) \mathrm{V} \subseteq \mathrm{UV} \subseteq \mathrm{U}$. Logo, $\left\{\mathrm{U}_{0}^{3} \mathrm{~V}_{0}+\left[\left(\mathrm{U}_{0} \mathrm{~V}_{0}\right) \mathrm{V}_{0}\right] \mathrm{V}_{0}\right\} \oplus \mathrm{U}_{0}\left(\mathrm{U}_{0} \mathrm{~V}_{0}\right) \subseteq\left\{\mathrm{U}^{3} \mathrm{~V}+\right.$ $+[(\mathrm{UV}) \mathrm{V}] \mathrm{V}\} \oplus \mathrm{U}(\mathrm{UV})$.

76. De (7.5) segue que $\left(\mathrm{U}_{0} \mathrm{~V}_{0}\right) \mathrm{V}_{0} \subseteq(\mathrm{UV}) \mathrm{V} \oplus(\mathrm{UV})^{2}=(\mathrm{UV}) \mathrm{V} \oplus$ $\oplus \mathrm{U}[(\mathrm{UV}) \mathrm{V}]$. De (7.8), temos $\mathrm{U}_{0}^{4} \subseteq(\mathrm{UV}) \mathrm{V} \oplus \mathrm{U}^{4}$ e finalmente, de (7.11), obtemos $\mathrm{U}_{0}\left[\left(\mathrm{U}_{0} \mathrm{~V}_{0}\right) \mathrm{V}_{0}\right] \oplus(\mathrm{UV}) \mathrm{V} \oplus \mathrm{U}[(\mathrm{UV}) \mathrm{V}]$. Portanto, $\left(\mathrm{U}_{0} \mathrm{~V}_{0}\right) \mathrm{V}_{0} \oplus$ $\oplus\left\{\mathrm{U}_{0}^{4}+\mathrm{U}_{0}\left[\left(\mathrm{U}_{0} \mathrm{~V}_{0}\right) \mathrm{V}_{0}\right]\right\} \subseteq(\mathrm{UV}) \mathrm{V} \oplus\left\{\mathrm{U}^{4}+\mathrm{U}[(\mathrm{UV}) \mathrm{V}]\right\}$.

77. Em (48) vimos que $\mathrm{U}_{0}\left[\mathrm{U}_{0}\left(\mathrm{U}_{0} \mathrm{~V}_{0}\right)\right] \oplus \mathrm{U}_{0}\left[\left(\mathrm{U}_{0} \mathrm{~V}_{0}\right) \mathrm{V}_{0}\right] \subseteq \mathrm{U}[\mathrm{U}(\mathrm{UV})] \oplus$ $\oplus \mathrm{U}[(\mathrm{UV}) \mathrm{V}]$. Além disso, de (7.5) segue que $\left(\mathrm{U}_{0} \mathrm{~V}_{0}\right) \mathrm{V}_{0} \subseteq(\mathrm{UV}) \mathrm{V} \oplus(\mathrm{UV})^{2}$ e portanto $\left\{\left(\mathrm{U}_{0} \mathrm{~V}_{0}\right) \mathrm{V}_{0}+\mathrm{U}_{0}\left[\mathrm{U}_{0}\left(\mathrm{U}_{0} \mathrm{~V}_{0}\right)\right]\right\} \oplus \mathrm{U}_{0}\left[\left(\mathrm{U}_{0} \mathrm{~V}_{0}\right) \mathrm{V}_{0}\right] \subseteq\{(\mathrm{UV}) \mathrm{V}+$ $+\mathrm{U}[\mathrm{U}(\mathrm{UV})]\} \oplus \mathrm{U}[(\mathrm{UV}) \mathrm{V}]$.

80. Por (46), $\mathrm{U}_{0}^{3} \mathrm{~V}_{0} \oplus \mathrm{U}_{0}^{4} \subseteq \mathrm{U}^{3} \mathrm{~V} \oplus \mathrm{U}^{4}$. Por outro lado, de (7.11) obtemos $\mathrm{U}_{0}\left[\left(\mathrm{U}_{0} \mathrm{~V}_{0}\right) \mathrm{V}_{0}\right] \subseteq(\mathrm{UV}) \mathrm{U}^{2} \oplus \mathrm{U}[(\mathrm{UV}) \mathrm{V}] \subseteq \mathrm{U}^{3} \mathrm{~V} \oplus\left\{\mathrm{U}^{4}+\mathrm{U}[(\mathrm{UV}) \mathrm{V}]\right\}$.

83. Da demonstração de (71) segue que $\left[\left(\mathrm{U}_{0} \mathrm{~V}_{0}\right) \mathrm{V}_{0}\right] \mathrm{V}_{0} \subseteq[(\mathrm{UV}) \mathrm{V}] \mathrm{V} \oplus$ $\oplus \mathrm{U}[(\mathrm{UV}) \mathrm{V}]$ e em $(48)$ mostramos que $\mathrm{U}_{0}\left[\mathrm{U}_{0}\left(\mathrm{U}_{0} \mathrm{~V}_{0}\right)\right] \oplus \mathrm{U}_{0}\left[\left(\mathrm{U}_{0} \mathrm{~V}_{0}\right) \mathrm{V}_{0}\right] \subseteq$ $\subseteq \mathrm{U}[\mathrm{U}(\mathrm{UV})] \oplus \mathrm{U}[(\mathrm{UV}) \mathrm{V}]$. Logo, $\left\{\mathrm{U}_{0}\left[\mathrm{U}_{0}\left(\mathrm{U}_{0} \mathrm{~V}_{0}\right)\right]+\left[\left(\mathrm{U}_{0} \mathrm{~V}_{0}\right) \mathrm{V}_{0}\right] \mathrm{V}_{0}\right\} \oplus$ $\oplus \mathrm{U}_{0}\left[\left(\mathrm{U}_{0} \mathrm{~V}_{0}\right) \mathrm{V}_{0}\right\} \subseteq\{\mathrm{U}[\mathrm{U}(\mathrm{UV})]+[(\mathrm{UV}) \mathrm{V}] \mathrm{V}\} \oplus \mathrm{U}[(\mathrm{UV}) \mathrm{V}]$.

89. Em (80) vimos que $\mathrm{U}_{0}^{3} \mathrm{~V}_{0} \ominus\left\{\mathrm{U}_{0}^{4}+\mathrm{U}_{0}\left[\left(\mathrm{U}_{0} \mathrm{~V}_{0}\right) \mathrm{V}_{0}\right]\right\} \subseteq \mathrm{U}^{3} \mathrm{~V} \ominus\left\{\mathrm{U}^{4}+\right.$ $+\mathrm{U}[(\mathrm{UV}) \mathrm{V}]\}$. Resta analisarmos $\left[\left(\mathrm{U}_{0} \mathrm{~V}_{0}\right) \mathrm{V}_{0}\right] \mathrm{V}_{0}$. Mas, novamente de (71). 
temos que $\left[\left(\mathrm{U}_{0} \mathrm{~V}_{0}\right) \mathrm{V}_{0}\right] \mathrm{V}_{0} \subseteq[(\mathrm{UV}) \mathrm{V}] \mathrm{V} \odot \mathrm{U}[(\mathrm{UV}) \mathrm{V}]$ e portanto $\left\{\mathrm{U}_{0}^{3} \mathrm{~V}_{0}+\right.$ $\left.+\left[\left(\mathrm{U}_{0} \mathrm{~V}_{0}\right) \mathrm{V}_{0}\right] \mathrm{V}_{0}\right\} \ominus\left\{\mathrm{U}_{0}^{4}+\mathrm{U}_{0}\left[\left(\mathrm{U}_{0} \mathrm{~V}_{0}\right) \mathrm{V}_{0}\right]\right\} \subseteq\left\{\mathrm{U}^{3} \mathrm{~V}+[(\mathrm{UV}) \mathrm{V}] \mathrm{V}\right\} \oplus$ $\oplus\left\{\mathrm{U}^{4}+\mathrm{U}[(\mathrm{UV}) \mathrm{V}]\right\}$.

Observemos que quando definimos os Grupos I e II, no Capítulo 5, dos 89 P-subespaços de grau $\leq 4,24$ pertenciam ao Grupo I e 65 ao Grupo II. No caso particular que acabamos de estudar, no Grupo I há 49 P-subespaços e no Grupo II há 40 P-subespaços.

\subsection{Problema 9: casos particulares}

Mais complexo do que a questão anterior é o problema inverso: fixado um determinado $\mathrm{P}$-subespaço, encontrar um subconjunto $\mathrm{S}_{P}$ do P-monômio $\mathrm{U}$, com $0 \in \mathrm{S}_{P}$, de modo que o P-subespaço $\mathrm{p}(\mathrm{U}, \mathrm{V})$ seja $\mathrm{S}_{P}$-invariante. Aqui, para cada um dos $\mathrm{P}$-subespaços do Grupo II, encontramos um subconjunto $\mathrm{S}$ de $\mathrm{S}_{P}$ para o qual $\mathrm{p}(\mathrm{U}, \mathrm{V})$ é S-invariante e em alguns casos foi possível determinar exatamente o conjunto $\mathrm{S}_{P}$.

Lema 7.2 Se $p(U, V)=U$, então $S_{P}=a n n_{U}\{U\}$.

Prova: De fato, temos $\mathrm{U}_{0}=\left\{\mathrm{u} \oplus 2 \lambda \mathrm{u}_{0} \mathrm{u}: \mathrm{u} \in \mathrm{U}\right\}$ e portanto $\mathrm{U}_{0} \subseteq \mathrm{U}$ se e somente se $\mathrm{u}_{0} \mathrm{u} \in \mathrm{U} \cap \mathrm{V}=\{0\}$ para todo $\mathrm{u} \in \mathrm{U}$ e isto ocorre se e somente se $\mathrm{u}_{0} \in \operatorname{ann}_{\mathrm{U}}\{\mathrm{U}\}$.

Lema 7.3 Se $p(U, V)=V$, então $S_{P}=\operatorname{ann}_{U}\{V\}$.

Prova: Como $\mathrm{V}_{0}=\left\{-2 \lambda \mathrm{u}_{0} \mathrm{v} \oplus \mathrm{v}: \mathrm{v} \in \mathrm{V}\right\}$, temos que $\mathrm{V}_{0} \subseteq \mathrm{UV} \oplus \mathrm{V}$ e portanto $\mathrm{V}_{0} \subseteq \mathrm{V}$ se e somente se $\mathrm{u}_{0} \mathrm{v}=0$ para todo $\mathrm{v} \in \mathrm{V}$; isto é, se e somente se $u_{0} \in \operatorname{ann}_{U}\{V\}$. 
Lema 7.4 Se $p(U, V)=U^{2}$, então $S_{P}=\operatorname{ann}_{U}\left\{U^{2}\right\}$.

Prova: Da Proposição 4, sabemos que o P-monômio $\mathrm{U}_{0}^{2}$ é gerado pelos elementos da forma $\mathrm{y}=-2 \lambda \mathrm{u}_{0}\left(\mathrm{uu}_{1}\right) \oplus \mathrm{uu}_{1}$ e portanto $\mathrm{y} \in \mathrm{U}^{2}$ se e somente se $\mathrm{u}_{0}\left(\mathrm{uu}_{1}\right)=0$, para todos $\mathrm{u}_{1} \mathrm{u}_{1} \in \mathrm{U}$, o que ocorre se e somente se $\mathrm{u}_{0} \in$ $\in \operatorname{ann}_{\mathrm{U}}\left\{\mathrm{U}^{2}\right\}$.

Lema 7.5 Se $p(U, V)=U V$, então $S_{P}=a n n_{U}\{U V\}$.

Prova: Um gerador de $U_{0} V_{0}$ é do tipo y $=\left[u v+2 \lambda^{2} u_{0}^{2}(u v)\right] \oplus$ $\oplus 2 \lambda \mathrm{u}_{0}(\mathrm{uv})$. Logo, $\mathrm{y} \in \mathrm{UV}$ se e somente se $\mathrm{u}_{0}(\mathrm{uv}) \in \mathrm{UV}$, para todos $\mathrm{u} \in \mathrm{U}, \mathrm{v} \in \mathrm{V}$ e isto ocorre se e somente se $\mathrm{u}_{0}(\mathrm{uv})=0$, para todos $\mathrm{u} \in \mathrm{U}$, $\mathrm{v} \in \mathrm{V}$; ou seja, se e somente se $\mathrm{u}_{0} \in \operatorname{ann}_{\mathrm{U}}\{\mathrm{UV}\}$.

Lema 7.6 Se $p(U, V)=U^{3}$, então $S_{P}=\operatorname{ann}_{U}\left\{U^{3}\right\}$.

Prova: De fato, pela Proposição 5 , temos que $\mathrm{U}_{0}^{3}$ é gerado por elementos da forma $\mathrm{y}=\left\{\mathrm{u}_{2}\left(\mathrm{uu}_{1}\right)+2 \lambda^{2} \mathrm{u}_{0}^{2}\left[\mathrm{u}_{2}\left(\mathrm{uu}_{1}\right)\right]\right\} \oplus 2 \lambda \mathrm{u}_{0}\left[\mathrm{u}_{2}\left(\mathrm{uu}_{1}\right)\right]$ e portanto $\mathrm{U}_{0}^{3} \subseteq \mathrm{U}^{3}$ se e somente se $\mathrm{u}_{0}\left[\mathrm{u}_{2}\left(\mathrm{uu}_{1}\right)\right] \in \mathrm{U}^{3} \cap \mathrm{V}=0$, para todos $\mathrm{u}, \mathrm{u}_{1}$, $\mathrm{u}_{2} \in \mathrm{U}$, isto é, se e somente se $\mathrm{u}_{0} \in \operatorname{ann}_{\mathrm{U}}\left\{\mathrm{U}^{3}\right\}$.

Lema 7.7 Se $S \subseteq \operatorname{ann}_{U}\{U(U V)\}$, então $U(U V)$ é S-invariante.

Prova: Consideremos $\mathrm{u}_{0} \in \mathrm{S}$ e mostremos que $\mathrm{U}_{0}\left(\mathrm{U}_{0} \mathrm{~V}_{0}\right) \subseteq \mathrm{U}(\mathrm{UV})$. Um gerador de $\mathrm{U}_{0}\left(\mathrm{U}_{0} \mathrm{~V}_{0}\right)$ é do tipo $\mathrm{y}=\left\{-2 \lambda \mathrm{u}_{0}\left[\mathrm{u}_{1}(\mathrm{uv})\right]+4 \lambda^{3} \mathrm{u}_{0}\left[\left(\mathrm{u}_{1} \mathrm{u}_{0}^{2}\right)(\mathrm{uv})\right]\right\} \oplus$ $\oplus\left\{u_{1}(u v)-2 \lambda^{2}\left(u_{1} u_{0}^{2}\right)(u v)\right\}$ (pela Proposição 2) e de $u_{0} \in a_{U}\{U(U V)\}$, segue que $\mathrm{u}_{0}\left[\mathrm{u}_{1}(\mathrm{uv})\right]=\mathrm{u}_{0}\left[\left(\mathrm{u}_{1} \mathrm{u}_{0}^{2}\right)(\mathrm{uv})\right]=0$, uma vez que $\left(\mathrm{u}_{1} \mathrm{u}_{0}^{2}\right)(\mathrm{uv}) \epsilon$ $\in \mathrm{U}^{3}(\mathrm{UV}) \subseteq \mathrm{U}(\mathrm{UV})$. Portanto, $\mathrm{y}=\mathrm{u}_{1}(\mathrm{uv})-2 \lambda^{2}\left(\mathrm{u}_{1} \mathrm{u}_{0}^{2}\right)(\mathrm{uv}) \in \mathrm{U}(\mathrm{UV}), \mathrm{o}$ que mostra que U(UV) é S-invariante.

Lema 7.8 Se $p(U, V)=(U V) V$, então $S_{P}=a n n_{U}\{(U V) V\}$. 
Prova: Seja y um gerador do P-subespaço $\left(\mathrm{U}_{0} \mathrm{~V}_{0}\right) \mathrm{V}_{0}$. Então, pela Proposição $3, \mathrm{y}=(\mathrm{uv}) \mathrm{v}_{1} \oplus 2 \lambda \mathrm{u}_{0}\left[(\mathrm{uv}) \mathrm{v}_{1}\right]$ e portanto $\mathrm{y} \in(\mathrm{UV}) \mathrm{V}$ se e somente se $u_{0}\left[(u v) v_{1}\right] \in(U V) V$ para todos $u \in U, v, v_{1} \in V$. Dessa forma, $y \in$ $\in(U V) V$ se e somente se $u_{0}\left[(u v) v_{1}\right]=0$, para todos $u \in U, v, v_{1} \in V$; isto é, se e somente se $\mathrm{u}_{0} \in \operatorname{ann}_{\mathrm{U}}\{(\mathrm{UV}) \mathrm{V}\}$.

Lema 7.9 Se $S \subseteq \operatorname{ann}_{U}\left\{U^{4}\right\}$, então $U^{4}$ é $S$-invariante.

Prova: Considerando $\mathrm{u}_{0} \in \mathrm{S}$, teremos $\mathrm{u}_{0}^{2}\left[\mathrm{u}_{2}\left(\mathrm{uu}_{1}\right)\right]=\mathrm{u}_{0}\left\{\mathrm{u}_{0}\left[\mathrm{u}_{2}\left(\mathrm{uu}_{1}\right)\right]\right\}=$ $=\mathrm{u}_{0}\left\{\mathrm{u}_{3}\left[\mathrm{u}_{2}\left(\mathrm{uu}_{1}\right)\right]\right\}=0$. Dessa forma, da Proposição 6, segue que um gerador de $U_{0}^{4}$ é da forma $u_{3}\left[u_{2}\left(u_{1}\right)\right]$, que obviamente é um elemento de $\mathrm{U}^{4}$ e portanto $\mathrm{U}_{0}^{4} \subseteq \mathrm{U}^{4}$, o que mostra que $\mathrm{U}^{4}$ é S-invariante.

Lema 7.10 Se $S \subseteq \operatorname{ann}_{U}\{U[U(U V)]\}$, então $U[U(U V)]$ é S-invariante.

Prova: Consideremos $\mathrm{u}_{0} \in \mathrm{S}$ e y um gerador de $\mathrm{U}_{0}\left[\mathrm{U}_{0}\left(\mathrm{U}_{0} \mathrm{~V}_{0}\right)\right]$. Então $\mathrm{u}_{0}\left\{\mathrm{u}_{2}\left[\mathrm{u}_{1}(\mathrm{uv})\right]\right\}=\mathrm{u}_{0}\left\{\mathrm{u}_{2}\left[\left(\mathrm{u}_{1} \mathrm{u}_{0}^{2}\right)(\mathrm{uv})\right]\right\}=0$ e como, além disso, $\mathrm{U}^{2}(\mathrm{UV})=$ $=\mathrm{U}^{3} \mathrm{~V} \subseteq \mathrm{U}[\mathrm{U}(\mathrm{UV})]$, segue que $\mathrm{u}_{0}\left[\left(\mathrm{u}_{0} \mathrm{u}_{1}\right)(\mathrm{uv})\right]=0$. Por outro lado, $\mathrm{u}_{0}^{2}\left\{\mathrm{u}_{2}\left[\mathrm{u}_{1}(\mathrm{uv})\right]\right\}=-2 \mathrm{u}_{0}\left\{\mathrm{u}_{0}\left\{\mathrm{u}_{2}\left[\mathrm{u}_{1}(\mathrm{uv})\right]\right\}\right\}=0$ e de $\mathrm{J}\left(\mathrm{u}_{0}, \mathrm{u}_{2}, \mathrm{u}_{0}\left[\left(\mathrm{u}_{1} \mathrm{u}_{0}^{2}\right)(\mathrm{uv})\right]\right)=$ $=0$, temos que $\mathrm{t}=\left(\mathrm{u}_{0} \mathrm{u}_{2}\right)\left\{\mathrm{u}_{0}\left[\left(\mathrm{u}_{1} \mathrm{u}_{0}^{2}\right)(\mathrm{uv})\right]\right\}=-\mathrm{u}_{0}\left\{\mathrm{u}_{2}\left\{\mathrm{u}_{0}\left[\left(\mathrm{u}_{1} \mathrm{u}_{0}^{2}\right)(\mathrm{uv})\right]\right\}\right\}$, uma vez que $\mathrm{u}_{0}\left\{\mathrm{u}_{0}\left[\left(\mathrm{u}_{1} \mathrm{u}_{0}^{2}\right)(\mathrm{uv})\right]\right\} \in \mathrm{u}_{0}\left(\mathrm{u}_{0} \mathrm{~V}\right)=0$. Da identidade (4.4) aplicada aos elementos $\mathrm{u}_{2}, \mathrm{u}_{0} \in \mathrm{U}$ e $\left(\mathrm{u}_{1} \mathrm{u}_{0}^{2}\right)(\mathrm{uv}) \in \mathrm{V}$, segue que $\mathrm{t}=$ $=\mathrm{u}_{0}\left\{\mathrm{u}_{0}\left\{\mathrm{u}_{2}\left[\left(\mathrm{u}_{1} \mathrm{u}_{0}^{2}\right)(\mathrm{uv})\right]\right\}\right\}=0$. Assim, pela Proposição 8, $\mathrm{y}=\mathrm{u}_{2}\left[\mathrm{u}_{1}(\mathrm{uv})\right] \epsilon$ $\in \mathrm{U}[\mathrm{U}(\mathrm{UV})]$ e dessa forma $\mathrm{U}_{0}\left[\mathrm{U}_{0}\left(\mathrm{U}_{0} \mathrm{~V}_{0}\right)\right] \subseteq \mathrm{U}[\mathrm{U}(\mathrm{UV})]$; ou seja, $\mathrm{U}[\mathrm{U}(\mathrm{UV})]$ é S-invariante.

Lema 7.11 Se $p(U, V)=U[(U V) V]$, então $S_{P}=a n n_{U}\{U[(U V) V]\}$.

Prova: De fato, pela Proposição 9, um gerador de $\mathrm{U}_{0}\left[\left(\mathrm{U}_{0} \mathrm{~V}_{0}\right) \mathrm{V}_{0}\right]$ é da forma $\mathrm{y}=-2 \lambda \mathrm{u}_{0}\left\{\mathrm{u}_{1}\left[(\mathrm{uv}) \mathrm{v}_{1}\right]\right\} \oplus \mathrm{u}_{1}\left[(\mathrm{uv}) \mathrm{v}_{1}\right]$ e assim temos $\mathrm{U}_{0}\left[\left(\mathrm{U}_{0} \mathrm{~V}_{0}\right) \mathrm{V}_{0}\right] \subseteq$ $\subseteq \mathrm{U}[(\mathrm{UV}) \mathrm{V}]$ se e somente se $\mathrm{u}_{0}\left\{\mathrm{u}_{1}\left[(\mathrm{uv}) \mathrm{v}_{1}\right]\right\} \in \mathrm{U}[(\mathrm{UV}) \mathrm{V}]$, para todos $\mathrm{u}$, $\mathrm{u}_{1} \in \mathrm{U}, \mathrm{v}, \mathrm{v}_{1} \in \mathrm{V}$, ou seja, se e somente se $\mathrm{u}_{0}\left\{\mathrm{u}_{1}\left[(\mathrm{uv}) \mathrm{v}_{1}\right]\right\}=0$, para todos 
$\mathrm{u}, \mathrm{u}_{1} \in \mathrm{U}, \mathrm{v}, \mathrm{v}_{1} \in \mathrm{V}$; isto é, se e somente se $\mathrm{u}_{0} \in \operatorname{ann}_{\mathrm{U}}\{\mathrm{U}[(\mathrm{UV}) \mathrm{V}]\}$.

Lema 7.12 Se $p(U, V)=U^{3} V$, então $S_{P}=a n n_{U}\left\{U^{3} V\right\}$.

Prova: Consideremos um gerador y do P-monômio $\mathrm{U}_{0}^{3} \mathrm{~V}_{0}$. Então y $=$ $=\left[\mathrm{u}_{2}\left(\mathrm{uu}_{1}\right)\right] \mathrm{v} \oplus 2 \lambda \mathrm{u}_{0}\left\{\left[\mathrm{u}_{2}\left(\mathrm{uu}_{1}\right)\right] \mathrm{v}\right\}$ e daí segue que $\mathrm{U}_{0}^{3} \mathrm{~V}_{0} \subseteq \mathrm{U}^{3} \mathrm{~V}$ se e somente se $\mathrm{u}_{0}\left\{\left[\mathrm{u}_{2}\left(\mathrm{uu}_{1}\right)\right] \mathrm{v}\right\}=0$, para todos $\mathrm{u}, \mathrm{u}_{1}, \mathrm{u}_{2} \in \mathrm{U}, \mathrm{v} \in \mathrm{V}$, o que ocorre se e somente se $\mathrm{u}_{0} \in \operatorname{ann} n_{\mathrm{U}}\left\{\mathrm{U}^{3} \mathrm{~V}\right\}$.

Lema 7.13 Se $p(U, V)=[(U V) V] V$, então $S_{P}=\operatorname{ann}_{U}\{[(U V) V] V\}$.

Prova: Pela Proposição 10, um gerador de $\left[\left(U_{0} V_{0}\right) V_{0}\right] V_{0}$ é da forma $\mathrm{y}=\left\{\left[(\mathrm{uv}) \mathrm{v}_{1}\right] \mathrm{v}_{2}+2 \lambda^{2} \mathrm{u}_{0}^{2}\left\{\left[(\mathrm{uv}) \mathrm{v}_{1}\right] \mathrm{v}_{2}\right\}\right\} \oplus 2 \lambda \mathrm{u}_{0}\left\{\left[(\mathrm{uv}) \mathrm{v}_{1}\right] \mathrm{v}_{2}\right\}$, e portanto segue que $\left[\left(\mathrm{U}_{0} \mathrm{~V}_{0}\right) \mathrm{V}_{0}\right] \mathrm{V}_{0} \subseteq[(\mathrm{UV}) \mathrm{V}] \mathrm{V}$ se e somente se $\mathrm{u}_{0}\left\{\left[(\mathrm{uv}) \mathrm{v}_{1}\right] \mathrm{v}_{2}\right\} \in$ $\in[(\mathrm{UV}) \mathrm{V}] \mathrm{V}$ para todos $\mathrm{u} \in \mathrm{U}, \mathrm{v}, \mathrm{v}_{1}, \mathrm{v}_{2} \in \mathrm{V}$ e isto ocorre se e somente se $\mathrm{u}_{0}\left\{\left[(\mathrm{uv}) \mathrm{v}_{1}\right] \mathrm{v}_{2}\right\}=0$, para todos $\mathrm{u} \in \mathrm{U}, \mathrm{v}, \mathrm{v}_{1}, \mathrm{v}_{2} \in \mathrm{V}$; em outras palavras, $\left[\left(\mathrm{U}_{0} \mathrm{~V}_{0}\right) \mathrm{V}_{0}\right] \mathrm{V}_{0} \subseteq[(\mathrm{UV}) \mathrm{V}] \mathrm{V}$ se e somente se $\mathrm{u}_{0} \in \operatorname{ann}_{\mathrm{U}}\{[(\mathrm{UV}) \mathrm{V}] \mathrm{V}\}$.

Observação 3: Das Proposições 1 a 10, é fácil ver que para todo Pmonômio $\mathrm{m}(\mathrm{U}, \mathrm{V})$, vale a inclusão $\mathrm{m}\left(\mathrm{U}_{0}, \mathrm{~V}_{0}\right) \subseteq \mathrm{m}(\mathrm{U}, \mathrm{V}) \oplus \mathrm{p}(\mathrm{U}, \mathrm{V})$, para conveniente $\mathrm{p}(\mathrm{U}, \mathrm{V})$.

Observação 4: Consideremos três subespaços vetoriais $\mathrm{A}, \mathrm{B}$ e $\mathrm{C}$ de uma mesma F-álgebra V. Afirmamos que $\operatorname{ann}_{\mathrm{A}}\{\mathrm{B}+\mathrm{C}\}=\operatorname{ann}_{\mathrm{A}}\{\mathrm{B}\} \cap$ $\cap \operatorname{ann}_{A}\{C\}$. De fato, denotando por $0_{B}$ e $0_{C}$ o vetor nulo de $V$ visto como elemento de $\mathrm{B}$ e de $\mathrm{C}$, respectivamente, e considerando elementos arbitrários $\mathrm{b} \in \mathrm{B}, \mathrm{c} \in \mathrm{C}$ e $\mathrm{x} \in \operatorname{ann}_{\mathrm{A}}\{\mathrm{B}+\mathrm{C}\}$, teremos $\mathrm{xb}=\mathrm{x}\left(\mathrm{b}+0_{\mathrm{C}}\right)=0$, pois $\mathrm{b}+0_{\mathrm{C}} \in \mathrm{B}+\mathrm{C}$. Logo, $\mathrm{x} \in \operatorname{ann}_{\mathrm{A}}\{\mathrm{B}\}$. Analogamente, mostra-se que $\mathrm{x} \in \operatorname{ann}_{\mathrm{A}}\{\mathrm{C}\}$ e portanto $\mathrm{x} \in \operatorname{ann}_{\mathrm{A}}\{\mathrm{B}\} \cap \operatorname{ann}_{\mathrm{A}}\{\mathrm{C}\}$. Reciprocamente, se $\mathrm{y} \in \operatorname{ann}_{\mathrm{A}}\{\mathrm{B}\} \cap \operatorname{ann}_{\mathrm{A}}\{\mathrm{C}\}$, considerando $\mathrm{x}=\mathrm{b}+\mathrm{c}$ um elemento qualquer de $\mathrm{B}+\mathrm{C}$, teremos $\mathrm{yx}=\mathrm{y}(\mathrm{b}+\mathrm{c})=\mathrm{yb}+\mathrm{yc}=0$ e daí concluimos que 
$\mathrm{y} \in \operatorname{ann}_{\mathrm{A}}\{\mathrm{B}+\mathrm{C}\}$.

Observação 5: Para facilitar a leitura do texto, nos lemas a seguir manteremos para cada P-subespaço a correspondente numeração com a qual ele foi listado no Grupo II (Capítulo 5, página 78).

Observação 6: É claro que se $\mathrm{S} \subseteq \mathrm{UV}$, então os P-subespaços listados no Lema 7.1 são S-invariantes.

Lema 7.14 Se $S \subseteq U^{3}$, então os seguintes P-subespaços do Grupo II são S-invariantes:

15. $U \oplus U^{4}$

18. $U^{3} \oplus V$

20. $U[U(U V)] \oplus V$

21. $U^{3} V \oplus V$

30. $U V \oplus U^{4}$

39. $U^{3} V \oplus U(U V)$

41. $(U V) V \oplus U^{4}$

46. $U^{3} V \oplus U^{4}$

56. $\{(U V) V+U[U(U V)]\} \oplus V$

61. $\{(U V) V+U[U(U V)]\} \oplus U^{2}$

67. $\left[U^{3}+(U V) V\right] \oplus U^{4}$

70. $\left\{U^{3}+[(U V) V] V\right\} \oplus U^{4}$

75. $\{(U V) V+U[U(U V)]\} \oplus U^{4}$ 


$$
\begin{aligned}
& \text { 79. }\{U[U(U V)]+[(U V) V] V\} \oplus U^{4} \\
& \text { 80. } U^{3} V \oplus\left\{U^{4}+U[(U V) V]\right\} \\
& \text { 82. }\left\{U^{3} V+[(U V) V] V\right\} \oplus U^{4} \\
& \text { 89. }\left\{U^{3} V+[(U V) V] V\right\} \oplus\left\{U^{4}+U[(U V) V]\right\}
\end{aligned}
$$

Prova: Comecemos observando que $\mathrm{U}^{3} \subseteq \mathrm{UV}$ e portanto, se $\mathrm{u}_{0} \in \mathrm{U}^{3}$, pelo Lema 7.1, os P-subespaços de números $39,46,56,61,80$ e 89 são invariantes.

15. Seja $\mathrm{u}_{0} \in \mathrm{S}$. Mostremos que $\mathrm{p}\left(\mathrm{U}_{0}, \mathrm{~V}_{0}\right) \subseteq \mathrm{p}(\mathrm{U}, \mathrm{V})$. De fato, se y é um gerador de $\mathrm{U}_{0}^{4}$, pela Proposição $6, \mathrm{y} \in \mathrm{U} \oplus \mathrm{U}^{4}$. Além disso, um gerador de $\mathrm{U}_{0}$ é da forma $\mathrm{u} \oplus 2 \lambda \mathrm{u}_{0} \mathrm{u}$ e como $\mathrm{u}_{0} \in \mathrm{U}^{3}$, segue que $\mathrm{u} \oplus$ $\oplus 2 \lambda \mathrm{u}_{0} \mathrm{u} \in \mathrm{U} \oplus \mathrm{U}^{4}$ e portanto $\mathrm{U}_{0} \oplus \mathrm{U}_{0}^{4} \subseteq \mathrm{U} \oplus \mathrm{U}^{4}$. Ou seja, $\mathrm{U} \oplus \mathrm{U}^{4}$ é S-invariante.

18. De fato, da Proposição 5 é fácil ver que $\mathrm{U}_{0}^{3} \subseteq \mathrm{U}^{3} \oplus \mathrm{V}$, para todo $\mathrm{u}_{0} \in$ $\in \mathrm{U}$. Assim, $\mathrm{U}_{0}^{3} \oplus \mathrm{V}_{0} \subseteq \mathrm{U}^{3} \oplus \mathrm{V}$ se e somente se $\mathrm{V}_{0} \subseteq \mathrm{U}^{3} \oplus \mathrm{V}$. Sejam, então, $\mathrm{u}_{0} \in \mathrm{S}$ e y um gerador de $\mathrm{V}_{0}$. Então, por (1.21), y $=-2 \lambda \mathrm{u}_{0} \mathrm{v} \oplus$ $\oplus \mathrm{v} \in \mathrm{U}^{3} \mathrm{~V} \oplus \mathrm{V} \subseteq \mathrm{U}^{3} \oplus \mathrm{V}$, o que mostra que $\mathrm{U}^{3} \oplus \mathrm{V}$ é um P-subespaço S-invariante.

20. A inclusão $\mathrm{U}_{0}\left[\mathrm{U}_{0}\left(\mathrm{U}_{0} \mathrm{~V}_{0}\right)\right] \subseteq \mathrm{U}[\mathrm{U}(\mathrm{UV})] \oplus \mathrm{V}$ é verdadeira para todo $\mathrm{u}_{0} \in \mathrm{U}$, pela Proposição 8. Consideremos agora $\mathrm{u}_{0} \in \mathrm{S}$ e $\mathrm{y}=-2 \lambda \mathrm{u}_{0} \mathrm{v} \oplus \mathrm{v}$ um gerador de $\mathrm{V}_{0}$. Então y $\in \mathrm{U}^{3} \mathrm{~V} \oplus \mathrm{V} \subseteq \mathrm{U}[\mathrm{U}(\mathrm{UV})] \oplus \mathrm{V}$, uma vez que $\mathrm{U}^{3} \mathrm{~V} \subseteq \mathrm{U}[\mathrm{U}(\mathrm{UV})]$. Dessa forma, $\mathrm{p}\left(\mathrm{U}_{0}, \mathrm{~V}_{0}\right) \subseteq \mathrm{p}(\mathrm{U}, \mathrm{V})$, para todo $\mathrm{u}_{0} \in \mathrm{S}$ e portanto $\mathrm{U}[\mathrm{U}(\mathrm{UV})] \oplus \mathrm{V}$ é S-invariante.

21. Seja $\mathrm{u}_{0} \in \mathrm{S}$. Da Proposição 7 obtemos a inclusão $\mathrm{U}_{0}^{3} \mathrm{~V}_{0} \subseteq \mathrm{U}^{3} \mathrm{~V} \oplus$ $\oplus \mathrm{V}$. Por outro lado, se $\mathrm{y} \in \mathrm{V}_{0}$, então $\mathrm{y}==-2 \lambda \mathrm{u}_{0} \mathrm{v} \oplus \mathrm{v} \in \mathrm{U}^{3} \mathrm{~V} \oplus \mathrm{V}$ (pois $\mathrm{u}_{0} \in \mathrm{U}^{3}$ ) e portanto $\mathrm{U}^{3} \mathrm{~V} \oplus \mathrm{V}$ é um P-subespaço S-invariante.

30. Analisando os geradores de $\mathrm{U}_{0}^{4}$ dados pela Proposição 6, concluimos que $\mathrm{U}_{0}^{4} \subseteq \mathrm{UV} \oplus \mathrm{U}^{4}$. Por outro lado, da Proposição 1 segue que $\mathrm{U}_{0} \mathrm{~V}_{0} \subseteq$ 
$\subseteq \mathrm{UV} \oplus \mathrm{u}_{0}(\mathrm{UV})$ e portanto se $\mathrm{u}_{0} \in \mathrm{S} \subseteq \mathrm{U}^{3}$, teremos $\mathrm{u}_{0}(\mathrm{UV}) \subseteq \mathrm{U}^{4}$ e daí obtemos $\mathrm{U}_{0} \mathrm{~V}_{0} \subseteq \mathrm{UV} \ominus \mathrm{U}^{4}$ : ou seja, $\mathrm{UV} \oplus \mathrm{U}^{4}$ é S-invariante.

41. Consideremos $\mathrm{u}_{0} \in \mathrm{S}$ e $\mathrm{y}_{1}, \mathrm{y}_{2}$ geradores de $\left(\mathrm{U}_{0} \mathrm{~V}_{0}\right) \mathrm{V}_{0}$ e $\mathrm{U}_{0}^{4}$, respectivamente. Pela Proposição $3, \mathrm{y}_{1} \in(\mathrm{UV}) \mathrm{V} \oplus \mathrm{u}_{0}[(\mathrm{UV}) \mathrm{V}] \subseteq(\mathrm{UV}) \mathrm{V} \oplus \mathrm{U}^{4}$, pois $\mathrm{u}_{0} \in \mathrm{U}^{3}$. Por outro lado, pela Proposição $6, \mathrm{y}_{2} \in\left[(\mathrm{UV}) \mathrm{V}+\mathrm{u}_{0} \mathrm{U}^{4}\right] \oplus$ $\oplus \mathrm{U}^{4}$ e como $\mathrm{u}_{0} \in \mathrm{U}^{3}=\mathrm{U}^{2} \mathrm{U} \subseteq \mathrm{UV}$, segue que $\mathrm{u}_{0} \mathrm{U}^{4} \subseteq$ (UV)V e portanto $\mathrm{y}_{2} \in(\mathrm{UV}) \mathrm{V} \oplus \mathrm{U}^{4}$. Logo, $\left(\mathrm{U}_{0} \mathrm{~V}_{0}\right) \mathrm{V}_{0} \oplus \mathrm{U}_{0}^{4} \subseteq(\mathrm{UV}) \mathrm{V} \oplus \mathrm{U}^{4}$ e segue daí que o P-subespaço (UV)V $\oplus \mathrm{U}^{4}$ é S-invariante.

67. Do Lema 2.6 sabemos que $\mathrm{U}^{3} \oplus \mathrm{U}^{4}$ é um P-subespaço invariante sendo, portanto, S-invariante para todo $\mathrm{S}$. Por outro lado, se $\mathrm{u}_{0} \in \mathrm{S}$ e y é um gerador de $\left(\mathrm{U}_{0} \mathrm{~V}_{0}\right) \mathrm{V}_{0}$, da Proposição 3 segue que $\mathrm{y} \in(\mathrm{UV}) \mathrm{V} \oplus$ $\oplus \mathrm{u}_{0}[(\mathrm{UV}) \mathrm{V}] \subseteq(\mathrm{UV}) \mathrm{V} \oplus \mathrm{U}^{4}$, pois $\mathrm{u}_{0} \in \mathrm{U}^{3}$. Assim, $\left[\mathrm{U}_{0}^{3}+\left(\mathrm{U}_{0} \mathrm{~V}_{0}\right) \mathrm{V}_{0}\right] \oplus$ $\oplus \mathrm{U}_{0}^{4} \subseteq\left[\left(\mathrm{U}^{3}+(\mathrm{UV}) \mathrm{V}\right] \oplus \mathrm{U}^{4}\right.$ e isto mostra que este último P-subespaço é S-invariante.

70. Como no caso anterior, resta analisarmos apenas o P-monômio $\left[\left(\mathrm{U}_{0} \mathrm{~V}_{0}\right) \mathrm{V}_{0}\right] \mathrm{V}_{0}$. Sejam $\mathrm{u}_{0} \in \mathrm{S}$ e y um gerador de $\left[\left(\mathrm{U}_{0} \mathrm{~V}_{0}\right) \mathrm{V}_{0}\right] \mathrm{V}_{0}$. Pela Proposição $10, \mathrm{y} \in[(\mathrm{UV}) \mathrm{V}] \mathrm{V} \oplus \mathrm{u}_{0}\{[(\mathrm{UV}) \mathrm{V}] \mathrm{V}\} \subseteq[(\mathrm{UV}) \mathrm{V}] \mathrm{V} \oplus \mathrm{U}^{4}$, uma vez que $\mathrm{u}_{0} \in \mathrm{U}^{3}$. Logo, $\mathrm{p}\left(\mathrm{U}_{0}, \mathrm{~V}_{0}\right) \subseteq \mathrm{p}(\mathrm{U}, \mathrm{V})$ e portanto $\mathrm{p}(\mathrm{U}, \mathrm{V})$ é S-invariante.

75. Já vimos que $\left(\mathrm{U}_{0} \mathrm{~V}_{0}\right) \mathrm{V}_{0} \oplus \mathrm{U}_{0}^{4}$ é S-invariante (vide (41)). Consideremos agora $\mathrm{u}_{0} \in \mathrm{S}$ e y um gerador de $\mathrm{U}_{0}\left[\mathrm{U}_{0}\left(\mathrm{U}_{0} \mathrm{~V}_{0}\right)\right]$. Da Proposição 8 temos que y $\in \mathrm{U}[\mathrm{U}(\mathrm{UV})] \oplus \mathrm{u}_{0}\{\mathrm{U}[\mathrm{U}(\mathrm{UV})]\} \subseteq \mathrm{U}[\mathrm{U}(\mathrm{UV})] \oplus \mathrm{U}^{4}$, pois $\mathrm{u}_{0} \in \mathrm{U}^{3}$. Dessa forma, $\left\{\left(\mathrm{U}_{0} \mathrm{~V}_{0}\right) \mathrm{V}_{0}+\mathrm{U}_{0}\left[\mathrm{U}_{0}\left(\mathrm{U}_{0} \mathrm{~V}_{0}\right)\right]\right\} \oplus \mathrm{U}_{0}^{4} \subseteq\{(\mathrm{UV}) \mathrm{V}+$ $+\mathrm{U}[\mathrm{U}(\mathrm{UV})]\} \oplus \mathrm{U}^{4}$, e portanto este último subespaço é S-invariante.

79. Seja $\mathrm{u}_{0} \in \mathrm{S}$. No caso anterior ficou mostrado que $\left.\mathrm{U}_{0}\left[\mathrm{U}_{0}\left(\mathrm{U}_{0} \mathrm{~V}_{0}\right)\right]\right\} \subseteq$ $\subseteq \mathrm{U}[\mathrm{U}(\mathrm{UV})] \oplus \mathrm{U}^{4}$. Já em (70) mostramos a inclusão $\left[\left(\mathrm{U}_{0} \mathrm{~V}_{0}\right) \mathrm{V}_{0}\right] \mathrm{V}_{0} \subseteq$ $\subseteq[(\mathrm{UV}) \mathrm{V}] \mathrm{V} \oplus \mathrm{U}^{4}$. Finalmente, se y é um gerador de $\mathrm{U}_{0}^{4}$, pela Proposição 6 e de $\mathrm{u}_{0} \in \mathrm{U}^{3}$, temos que $\mathrm{y} \in \mathrm{u}_{0} \mathrm{U}^{4} \oplus \mathrm{U}^{4} \subseteq \mathrm{U}^{3} \mathrm{~V} \oplus \mathrm{U}^{4} \subseteq \mathrm{U}[\mathrm{U}(\mathrm{UV})] \oplus$ $\oplus \mathrm{U}^{4}$ (sendo esta inclusão consequência do diagrama da página 9). Logo, 
$\mathrm{p}\left(\mathrm{U}_{0}, \mathrm{~V}_{0}\right) \subseteq \mathrm{p}(\mathrm{U}, \mathrm{V})$ e portanto $\mathrm{p}(\mathrm{U}, \mathrm{V})$ é S-invariante.

82. Consideremos $\mathrm{u}_{0} \in \mathrm{S}$. De (46), temos que $\mathrm{U}_{0}^{3} \mathrm{~V}_{0} \oplus \mathrm{U}_{0}^{4} \subseteq \mathrm{U}^{3} \mathrm{~V} \oplus$ $\oplus \mathrm{U}^{4}$. Basta, então, analisarmos o P-monômio $\left[\left(\mathrm{U}_{0} \mathrm{~V}_{0}\right) \mathrm{V}_{0}\right] \mathrm{V}_{0}$. Mas em (70) já vimos que $\left[\left(\mathrm{U}_{0} \mathrm{~V}_{0}\right) \mathrm{V}_{0}\right] \mathrm{V}_{0} \subseteq[(\mathrm{UV}) \mathrm{V}] \mathrm{V} \oplus \mathrm{U}^{4}$, o que implica em $\mathrm{p}\left(\mathrm{U}_{0}, \mathrm{~V}_{0}\right) \subseteq \mathrm{p}(\mathrm{U}, \mathrm{V})$ e portanto $\mathrm{p}(\mathrm{U}, \mathrm{V})$ é S-invariante.

Lema 7.15 Se $S \subseteq(U V) V$, então os seguintes P-subespaços do Grupo II são S-invariantes:

16. $U \oplus U[(U V) V]$

22. $[(U V) V] V \oplus V$

28. $[(U V) V] V \oplus U^{2}$

40. $[(U V) V] V \oplus U(U V)$

51. $[(U V) V] V \oplus U[(U V) V]$

81. $[(U V) V] V \oplus\left\{U^{4}+U[(U V) V]\right\}$

Prova: 16. Da Proposição 9 segue que $\mathrm{U}_{0}\left[\left(\mathrm{U}_{0} \mathrm{~V}_{0}\right) \mathrm{V}_{0}\right] \subseteq \mathrm{U} \oplus \mathrm{U}[(\mathrm{UV}) \mathrm{V}]$. Resta analisarmos o P-monômio $\mathrm{U}_{0}$. Mas se $\mathrm{u}_{0} \in(\mathrm{UV}) \mathrm{V}$ e y é um gerador de $\mathrm{U}_{0}$, então $\mathrm{y}=\mathrm{u} \oplus 2 \lambda \mathrm{u}_{0} \mathrm{u} \in \mathrm{U} \oplus \mathrm{U}[(\mathrm{UV}) \mathrm{V}]$ e portanto $\mathrm{p}\left(\mathrm{U}_{0}, \mathrm{~V}_{0}\right) \subseteq$ $\subseteq \mathrm{p}(\mathrm{U}, \mathrm{V})$, o que implica em $\mathrm{p}(\mathrm{U}, \mathrm{V})$ ser S-invariante.

22. Temos que $\left[\left(\mathrm{U}_{0} \mathrm{~V}_{0}\right) \mathrm{V}_{0}\right] \mathrm{V}_{0} \subseteq[(\mathrm{UV}) \mathrm{V}] \mathrm{V} \oplus \mathrm{V}$, para todo $\mathrm{u}_{0} \in \mathrm{U}$ (pela Proposição 10). Além disso, se $\mathrm{u}_{0} \in \mathrm{S}$ e y é um gerador de $\mathrm{V}_{0}$, então $\mathrm{y}=$ $=-2 \lambda \mathrm{u}_{0} \mathrm{v} \oplus \mathrm{v} \in[(\mathrm{UV}) \mathrm{V}] \mathrm{V} \oplus \mathrm{V}$, o que implica em $\left[\left(\mathrm{U}_{0} \mathrm{~V}_{0}\right) \mathrm{V}_{0}\right] \mathrm{V}_{0} \oplus \mathrm{U}_{0} \subseteq$ $\subseteq[(\mathrm{UV}) \mathrm{V}] \mathrm{V} \oplus \mathrm{V}$ e portanto $[(\mathrm{UV}) \mathrm{V}] \mathrm{V} \oplus \mathrm{V}$ é S-invariante.

28. A inclusão $\left[\left(\mathrm{U}_{0} \mathrm{~V}_{0}\right) \mathrm{V}_{0}\right] \mathrm{V}_{0} \subseteq[(\mathrm{UV}) \mathrm{V}] \mathrm{V} \oplus \mathrm{U}^{2}$ é sempre verdadeira, pela Proposição 10. Por outro lado, dado $\mathrm{u}_{0} \in \mathrm{S}$, pela Proposição 4 , um gerador de $\mathrm{U}_{0}^{2}$ é da forma $\mathrm{y}=-2 \lambda \mathrm{u}_{0}\left(\mathrm{uu}_{1}\right) \oplus \mathrm{uu}_{1}$. Como $\mathrm{u}_{0} \in(\mathrm{UV}) \mathrm{V}$, 
segue que $\mathrm{u}_{0}\left(\mathrm{uu}_{1}\right) \in[(\mathrm{UV}) \mathrm{V}] \mathrm{U}^{2} \subseteq[(\mathrm{UV}) \mathrm{V}] \mathrm{V}$ e assim $\mathrm{y} \in[(\mathrm{UV}) \mathrm{V}] \mathrm{V} \oplus$ $\ominus \mathrm{U}^{2}$, o que mostra que $[(\mathrm{UV}) \mathrm{V}] \mathrm{V} \oplus \mathrm{U}^{2}$ é um P-subespaço S-invariante.

40. Pela Proposição 10, a inclusão $\left[\left(\mathrm{U}_{0} \mathrm{~V}_{0}\right) \mathrm{V}_{0}\right] \mathrm{V}_{0} \subseteq[(\mathrm{UV}) \mathrm{V}] \mathrm{V} \oplus \mathrm{U}(\mathrm{UV})$ vale sempre. Consideremos agora $\mathrm{u}_{0} \in \mathrm{S}$ e y um gerador de $\mathrm{U}_{0}\left(\mathrm{U}_{0} \mathrm{~V}_{0}\right)$. Então, pela Proposição 2, y $\in \mathrm{u}_{0}[\mathrm{U}(\mathrm{UV})] \oplus \mathrm{U}(\mathrm{UV}) \subseteq \mathrm{u}_{0} \mathrm{~V} \oplus \mathrm{U}(\mathrm{UV}) \subseteq$ $\subseteq[(\mathrm{UV}) \mathrm{V}] \mathrm{V} \oplus \mathrm{U}(\mathrm{UV})$, pois $\mathrm{u}_{0} \in(\mathrm{UV}) \mathrm{V}$. Dessa forma, $\left[\left(\mathrm{U}_{0} \mathrm{~V}_{0}\right) \mathrm{V}_{0}\right] \mathrm{V}_{0} \oplus$ $\oplus \mathrm{U}_{0}\left(\mathrm{U}_{0} \mathrm{~V}_{0}\right) \subseteq[(\mathrm{UV}) \mathrm{V}] \mathrm{V} \oplus \mathrm{U}(\mathrm{UV})$, ou seja, $\mathrm{p}(\mathrm{U}, \mathrm{V})$ é S-invariante.

51. A inclusão $\left[\left(\mathrm{U}_{0} \mathrm{~V}_{0}\right) \mathrm{V}_{0}\right] \mathrm{V}_{0} \subseteq[(\mathrm{UV}) \mathrm{V}] \mathrm{V} \oplus \mathrm{U}[(\mathrm{UV}) \mathrm{V}]$ é sempre verdadeira, pela Proposição 10. Por outro lado, consideremos $u_{0} \in \mathrm{S}$. Pela Proposição 9 segue que $\mathrm{U}_{0}\left[\left(\mathrm{U}_{0} \mathrm{~V}_{0}\right) \mathrm{V}_{0}\right] \subseteq \mathrm{u}_{0}\{\mathrm{U}[(\mathrm{UV}) \mathrm{V}]\} \oplus \mathrm{U}[(\mathrm{UV}) \mathrm{V}] \subseteq$ $\subseteq[(\mathrm{UV}) \mathrm{V}] \mathrm{U}^{2} \oplus \mathrm{U}[(\mathrm{UV}) \mathrm{V}] \subseteq[(\mathrm{UV}) \mathrm{V}] \mathrm{V} \oplus \mathrm{U}[(\mathrm{UV}) \mathrm{V}]$, uma vez que $\mathrm{u}_{0} \in$ $\in(U V)$ V. Logo, $\mathrm{p}\left(\mathrm{U}_{0}, \mathrm{~V}_{0}\right) \subseteq \mathrm{p}(\mathrm{U}, \mathrm{V})$ e portanto $\mathrm{p}(\mathrm{U}, \mathrm{V})$ é S-invariante.

81. Usando o resultado do item anterior, basta mostrarmos que se $u_{0} \in$ $\mathrm{S}$, então $\mathrm{U}_{0}^{4} \subseteq[(\mathrm{UV}) \mathrm{V}] \mathrm{V} \oplus\left\{\mathrm{U}^{4}+\mathrm{U}[(\mathrm{UV}) \mathrm{V}]\right\}$. Mas da Proposição 6 segue que $\mathrm{U}_{0}^{4} \subseteq\left\{[(\mathrm{UV}) \mathrm{V}] \mathrm{V}+\mathrm{u}_{0} \mathrm{U}^{4}\right\} \oplus \mathrm{U}^{4} \subseteq[(\mathrm{UV}) \mathrm{V}] \mathrm{V} \oplus \mathrm{U}^{4}$, pois $\mathrm{u}_{0} \mathrm{U}^{4} \subseteq[(\mathrm{UV}) \mathrm{V}] \mathrm{U}^{4} \subseteq[(\mathrm{UV}) \mathrm{V}] \mathrm{V}$ e isto mostra que também o Psubespaço $[(U V) V] V \oplus\left\{\mathrm{U}^{4}+\mathrm{U}[(\mathrm{UV}) \mathrm{V}]\right\}$ é S-invariante.

Lema 7.16 Se $S \subseteq U^{3} \cup(U V) V$, então os seguintes $P$-subespaços do Grupo II são S-invariantes:

53. $U \oplus\left\{U^{4}+U[(U V) V]\right\}$

55. $\left\{U^{3}+[(U V) V] V\right\} \oplus V$

57. $\{U[U(U V)]+[(U V) V] V\} \oplus V$

58. $\left\{U^{3} V+[(U V) V] V\right\} \oplus V$

62. $\{U[U(U V)]+[(U V) V] V\} \oplus U^{2}$

63. $\left\{U^{3} V+[(U V) V] V\right\} \oplus U^{2}$

74. $\left\{U^{3} V+[(U V) V] V\right\} \oplus U(U V)$ 
Prova: 53. É claro que $\mathrm{U}_{0}^{4}+\mathrm{U}_{0}\left[\left(\mathrm{U}_{0} \mathrm{~V}_{0}\right) \mathrm{V}_{0}\right] \subseteq \mathrm{U} \oplus\left\{\mathrm{U}^{4}+\mathrm{U}[(\mathrm{UV}) \mathrm{V}]\right\}$ (veja Observação 3 , página 116). Por outro lado, dado um gerador y do P-monômio $\mathrm{U}_{0}$, de (1.20) segue que $\mathrm{y} \in \mathrm{U} \oplus \mathrm{u}_{0} \mathrm{U}$. Dessa forma, se $\mathrm{u}_{0} \in$ $\in \mathrm{U}^{3}$, então $\mathrm{u}_{0} \mathrm{U} \subseteq \mathrm{U}^{4}$ e se $\mathrm{u}_{0} \in(\mathrm{UV}) \mathrm{V}$ teremos $\mathrm{u}_{0} \mathrm{U} \in \mathrm{U}[(\mathrm{UV}) \mathrm{V}]$. Logo, para todo $\mathrm{u}_{0} \in \mathrm{S}$, temos $\mathrm{U}_{0} \subseteq \mathrm{U} \oplus\left\{\mathrm{U}^{4}+\mathrm{U}[(\mathrm{UV}) \mathrm{V}]\right\}$, o que mostra que este último P-subespaço é S-invariante.

55, 57 e 58. Novamente pela Observação 3 acima citada, obtemos as três seguintes inclusões: $\mathrm{U}_{0}^{3}+\left[\left(\mathrm{U}_{0} \mathrm{~V}_{0}\right) \mathrm{V}_{0}\right] \mathrm{V}_{0} \subseteq\left\{\mathrm{U}^{3}+[(\mathrm{UV}) \mathrm{V}] \mathrm{V}\right\} \oplus$ $\oplus \mathrm{V} ; \mathrm{U}_{0}\left[\mathrm{U}_{0}\left(\mathrm{U}_{0} \mathrm{~V}_{0}\right)\right]+\left[\left(\mathrm{U}_{0} \mathrm{~V}_{0}\right) \mathrm{V}_{0}\right] \mathrm{V}_{0} \subseteq\{\mathrm{U}[\mathrm{U}(\mathrm{UV})]+[(\mathrm{UV}) \mathrm{V}] \mathrm{V}\} \oplus \mathrm{V}$ e $\mathrm{U}_{0}^{3} \mathrm{~V}_{0}+\left[\left(\mathrm{U}_{0} \mathrm{~V}_{0}\right) \mathrm{V}_{0}\right] \mathrm{V}_{0} \subseteq\left\{\mathrm{U}^{3} \mathrm{~V}+[(\mathrm{UV}) \mathrm{V}] \mathrm{V}\right\} \oplus \mathrm{V}$. Considerando, agora, $\mathrm{u}_{0} \in \mathrm{S}$ e y um gerador de $\mathrm{V}_{0}$, de (1.21) segue que $\mathrm{y} \in \mathrm{u}_{0} \mathrm{~V} \oplus$ $\oplus \mathrm{V}$. Dessa forma, se $\mathrm{u}_{0} \in \mathrm{U}^{3}$ então $\mathrm{u}_{0} \mathrm{~V} \subseteq \mathrm{U}^{3} \mathrm{~V} \subseteq \mathrm{U}[\mathrm{U}(\mathrm{UV})] \subseteq \mathrm{U}^{3}$. Por outro lado, se $\mathrm{u}_{0} \in(\mathrm{UV}) \mathrm{V}$, teremos $\mathrm{u}_{0} \mathrm{~V} \subseteq[(\mathrm{UV}) \mathrm{V}] \mathrm{V}$ e daí concluimos que $\left\{\mathrm{U}_{0}^{3}+\left[\left(\mathrm{U}_{0} \mathrm{~V}_{0}\right) \mathrm{V}_{0}\right] \mathrm{V}_{0}\right\} \oplus \mathrm{V}_{0} \subseteq\left\{\mathrm{U}^{3}+[(\mathrm{UV}) \mathrm{V}] \mathrm{V}\right\} \oplus \mathrm{V}$; $\left\{\mathrm{U}_{0}\left[\mathrm{U}_{0}\left(\mathrm{U}_{0} \mathrm{~V}_{0}\right)\right]+\left[\left(\mathrm{U}_{0} \mathrm{~V}_{0}\right) \mathrm{V}_{0}\right] \mathrm{V}_{0}\right\} \oplus \mathrm{V}_{0} \subseteq\{\mathrm{U}[\mathrm{U}(\mathrm{UV})]+[(\mathrm{UV}) \mathrm{V}] \mathrm{V}\} \oplus$ $\oplus \mathrm{V}$ e $\left\{\mathrm{U}_{0}^{3} \mathrm{~V}_{0}+\left[\left(\mathrm{U}_{0} \mathrm{~V}_{0}\right) \mathrm{V}_{0}\right] \mathrm{V}_{0}\right\} \oplus \mathrm{V}_{0} \subseteq\left\{\mathrm{U}^{3} \mathrm{~V}+[(\mathrm{UV}) \mathrm{V}] \mathrm{V}\right\} \oplus \mathrm{V}$, o que mostra que os $\mathrm{P}$-subespaços de números 55,57 e 58 são S-invariantes.

62 e 63. Das Proposições 8 e 10 , obtemos a inclusão $\mathrm{U}_{0}\left[\mathrm{U}_{0}\left(\mathrm{U}_{0} \mathrm{~V}_{0}\right)\right]+$ $+\left[\left(\mathrm{U}_{0} \mathrm{~V}_{0}\right) \mathrm{V}_{0}\right] \mathrm{V}_{0} \subseteq\{\mathrm{U}[\mathrm{U}(\mathrm{UV})]+[(\mathrm{UV}) \mathrm{V}] \mathrm{V}\} \oplus \mathrm{U}^{2}$. Por outro lado, das Proposições 7 e 10 , segue a inclusão $\mathrm{U}_{0}^{3} \mathrm{~V}_{0}+\left[\left(\mathrm{U}_{0} \mathrm{~V}_{0}\right) \mathrm{V}_{0}\right] \mathrm{V}_{0} \subseteq$ $\subseteq\left\{\mathrm{U}^{3} \mathrm{~V}+[(\mathrm{UV}) \mathrm{V}] \mathrm{V}\right\} \oplus \mathrm{U}^{2}$. Consideremos, agora, $\mathrm{u}_{0} \in \mathrm{S}$ e y um gerador de $\mathrm{U}_{0}^{2}$. Então, pela Proposição $4, \mathrm{y} \in \mathrm{u}_{0} \mathrm{U}^{2} \oplus \mathrm{U}^{2}$. Assim, se $\mathrm{u}_{0} \in$ $\in \mathrm{U}^{3}$, teremos $\mathrm{u}_{0} \mathrm{U}^{2} \subseteq \mathrm{U}^{3} \mathrm{U}^{2} \subseteq \mathrm{U}^{3} \mathrm{~V} \subseteq \mathrm{U}[\mathrm{U}(\mathrm{UV})]$. Além disso, se $\mathrm{u}_{0} \in$ $\in(\mathrm{UV}) \mathrm{V}$, então $\mathrm{u}_{0} \mathrm{U}^{2} \subseteq[(\mathrm{UV}) \mathrm{V}] \mathrm{U}^{2} \subseteq[(\mathrm{UV}) \mathrm{V}] \mathrm{V}$. Em outras palavras, $\left\{\mathrm{U}_{0}\left[\mathrm{U}_{0}\left(\mathrm{U}_{0} \mathrm{~V}_{0}\right)\right]+\left[\left(\mathrm{U}_{0} \mathrm{~V}_{0}\right) \mathrm{V}_{0}\right] \mathrm{V}_{0}\right\} \oplus \mathrm{U}_{0}^{2} \subseteq\{\mathrm{U}[\mathrm{U}(\mathrm{UV})]+[(\mathrm{UV}) \mathrm{V}] \mathrm{V}\} \oplus$ $\oplus \mathrm{U}^{2}$ e $\left\{\mathrm{U}_{0}^{3} \mathrm{~V}_{0}+\left[\left(\mathrm{U}_{0} \mathrm{~V}_{0}\right) \mathrm{V}_{0}\right] \mathrm{V}_{0}\right\} \oplus \mathrm{U}_{0}^{2} \subseteq\left\{\mathrm{U}^{3} \mathrm{~V}+[(\mathrm{UV}) \mathrm{V}] \mathrm{V}\right\} \oplus \mathrm{U}^{2}, o$ que mostra que os P-subespaços de números 62 e 63 são S-invariantes.

74. Novamente das Proposições 7 e 10 , obtemos $\mathrm{U}_{0}^{3} \mathrm{~V}_{0}+\left[\left(\mathrm{U}_{0} \mathrm{~V}_{0}\right) \mathrm{V}_{0}\right] \mathrm{V}_{0} \subseteq$ $\subseteq\left\{\mathrm{U}^{3} \mathrm{~V}+[(\mathrm{UV}) \mathrm{V}] \mathrm{V}\right\} \oplus \mathrm{U}(\mathrm{UV})$. Sejam, agora, $\mathrm{u}_{0} \in \mathrm{S}$ e y um gerador de $\mathrm{U}_{0}\left(\mathrm{U}_{0} \mathrm{~V}_{0}\right)$. Da Proposição 2 segue que $\mathrm{y} \in \mathrm{u}_{0}[\mathrm{U}(\mathrm{UV})] \oplus \mathrm{U}(\mathrm{UV})$. Assim, se $\mathrm{u}_{0} \in \mathrm{U}^{3}$, teremos $\mathrm{u}_{0}[\mathrm{U}(\mathrm{UV})] \subseteq \mathrm{U}^{3}[\mathrm{U}(\mathrm{UV})] \subseteq \mathrm{U}^{3} \mathrm{~V}$ e se por outro lado 
$\mathrm{u}_{0} \in(\mathrm{UV}) \mathrm{V}$, então $\mathrm{u}_{0}[\mathrm{U}(\mathrm{UV})] \subseteq[(\mathrm{UV}) \mathrm{V}][\mathrm{U}(\mathrm{UV})] \subseteq[(\mathrm{UV}) \mathrm{V}] \mathrm{V}$. Logo, $\left\{\mathrm{U}_{0}^{3} \mathrm{~V}_{0}+\left[\left(\mathrm{U}_{0} \mathrm{~V}_{0}\right) \mathrm{V}_{0}\right] \mathrm{V}_{0}\right\} \ominus \mathrm{U}_{0}\left(\mathrm{U}_{0} \mathrm{~V}_{0}\right) \subseteq\left\{\mathrm{U}^{3} \mathrm{~V}+[(\mathrm{UV}) \mathrm{V}] \mathrm{V}\right\} \ominus \mathrm{U}(\mathrm{UV})$, o que mostra que este último subespaço é S-invariante.

Lema 7.17 Se $p(U, V)=U^{3}+(U V) V$ então $S_{P}=a n n_{U}\left\{U^{3}\right\} \cap$ $\cap a n n_{U}\{(U V) V\}$.

Prova: De fato, pelas Proposições 3 e 5, obtemos $\mathrm{U}_{0}^{3}+\left(\mathrm{U}_{0} \mathrm{~V}_{0}\right) \mathrm{V}_{0} \subseteq$ $\subseteq\left(\mathrm{U}^{3} \oplus \mathrm{u}_{0} \mathrm{U}^{3}\right)+\left\{(\mathrm{UV}) \mathrm{V} \oplus \mathrm{u}_{0}[(\mathrm{UV}) \mathrm{V}]\right\} \subseteq \mathrm{U}^{3}+(\mathrm{UV}) \mathrm{V}$ se e somente se $0=\mathrm{u}_{0}\left[\mathrm{U}^{3}+(\mathrm{UV}) \mathrm{V}\right]$; isto é, se e somente se $\mathrm{u}_{0} \in \operatorname{ann}_{\mathrm{U}}\left\{\mathrm{U}^{3}+(\mathrm{UV}) \mathrm{V}\right\}=$ $=\operatorname{ann}_{U}\left\{U^{3}\right\} \cap \operatorname{ann}_{U}\{(U V) V\}$.

Lema 7.18 Se $p(U, V)=U^{3}+[(U V) V] V$ então $S_{P}=a_{U} n_{U}\left\{U^{3}\right\} \cap$ $\cap \operatorname{ann}_{U}\{[(U V) V] V\}$.

Prova: De fato, pelas Proposições 5 e 10 , obtemos $\mathrm{U}_{0}^{3}+\left[\left(\mathrm{U}_{0} \mathrm{~V}_{0}\right) \mathrm{V}_{0}\right] \mathrm{V}_{0} \subseteq$ $\subseteq\left(\mathrm{U}^{3} \oplus \mathrm{u}_{0} \mathrm{U}^{3}\right)+\left\{[(\mathrm{UV}) \mathrm{V}] \mathrm{V} \oplus \mathrm{u}_{0}\{[(\mathrm{UV}) \mathrm{V}] \mathrm{V}\}\right\} \subseteq\left\{\mathrm{U}^{3}+[(\mathrm{UV}) \mathrm{V}] \mathrm{V}\right\} \oplus$ $\oplus \mathrm{u}_{0}\left\{\mathrm{U}^{3}+[(\mathrm{UV}) \mathrm{V}] \mathrm{V}\right\} \subseteq \mathrm{U}^{3}+[(\mathrm{UV}) \mathrm{V}] \mathrm{V}$ se e somente se $0=$ $=\mathrm{u}_{0}\left\{\left[\mathrm{U}^{3}+[(\mathrm{UV}) \mathrm{V}] \mathrm{V}\right\} ;\right.$ isto é, se e somente se $\mathrm{u}_{0} \in \operatorname{ann}_{\mathrm{U}}\left\{\mathrm{U}^{3}+[(\mathrm{UV}) \mathrm{V}] \mathrm{V}\right\}$ ou seja, se e somente se $\mathrm{u}_{0} \in \operatorname{ann}_{U}\left\{\mathrm{U}^{3}\right\} \cap \operatorname{ann}_{\mathrm{U}}\{[(\mathrm{UV}) \mathrm{V}] \mathrm{V}\}$.

Lema 7.19 Se $p(U, V)=(U V) V+U[U(U V)]$ então $S_{P}=$ $=\operatorname{ann}_{U}\{(U V) V\} \cap \operatorname{ann}_{U}\{U[U(U V)]\}$.

Prova: Inicialmente observemos que, pelas Proposições 3 e 8 , respectivamente, temos $\left(\mathrm{U}_{0} \mathrm{~V}_{0}\right) \mathrm{V}_{0} \subseteq(\mathrm{UV}) \mathrm{V} \oplus \mathrm{u}_{0}[(\mathrm{UV}) \mathrm{V}]$ e $\mathrm{U}_{0}\left[\mathrm{U}_{0}\left(\mathrm{U}_{0} \mathrm{~V}_{0}\right)\right] \subseteq$ $\subseteq \mathrm{U}[\mathrm{U}(\mathrm{UV})] \oplus \mathrm{u}_{0}\{\mathrm{U}[\mathrm{U}(\mathrm{UV})]\}$. Dessa forma, $\mathrm{u}_{0} \in \mathrm{S}_{\mathrm{P}}$ se e somente se $\left(\mathrm{U}_{0} \mathrm{~V}_{0}\right) \mathrm{V}_{0}+\mathrm{U}_{0}\left[\mathrm{U}_{0}\left(\mathrm{U}_{0} \mathrm{~V}_{0}\right)\right] \subseteq(\mathrm{UV}) \mathrm{V}+\mathrm{U}[\mathrm{U}(\mathrm{UV})]$ e isto ocorre se e somente se $\mathrm{u}_{0}[(\mathrm{UV}) \mathrm{V}]+\mathrm{u}_{0}\{\mathrm{U}[\mathrm{U}(\mathrm{UV})]\}=\mathrm{u}_{0}\{(\mathrm{UV}) \mathrm{V}+\mathrm{U}[\mathrm{U}(\mathrm{UV})]\}=0$; isto é, se e somente se $\mathrm{u}_{0} \in \operatorname{ann}_{\mathrm{U}}\{(\mathrm{UV}) \mathrm{V}+\mathrm{U}[\mathrm{U}(\mathrm{UV})]\}=\operatorname{ann}_{\mathrm{U}}\{(\mathrm{UV}) \mathrm{V}\} \cap$ $\cap \operatorname{ann}_{\mathrm{U}}\{\mathrm{U}[\mathrm{U}(\mathrm{UV})]\}$. 
Lema 7.20 Se $S \subseteq(U V) V \cap U^{3}$ entẫo $p(U, V)=[(U V) V] V \oplus U^{4} \dot{e}$ S-invariante.

Prova: Seja $\mathrm{u}_{0} \in \mathrm{S}$. Da Proposição 10, temos que $\left[\left(\mathrm{U}_{0} \mathrm{~V}_{0}\right) \mathrm{V}_{0}\right] \mathrm{V}_{0} \subseteq$ $\subseteq[(\mathrm{UV}) \mathrm{V}] \mathrm{V} \oplus \mathrm{u}_{0}\{[(\mathrm{UV}) \mathrm{V}] \mathrm{V}\} \subseteq[(\mathrm{UV}) \mathrm{V}] \mathrm{V} \oplus \mathrm{U}^{4}$, uma vez que $\mathrm{u}_{0} \in$ $\in \mathrm{U}^{3}$. Por outro lado, da Proposição 6 segue que $\mathrm{U}_{0}^{4} \subseteq\{[(\mathrm{UV}) \mathrm{V}] \mathrm{V}+$ $\left.+\mathrm{u}_{0} \mathrm{U}^{4}\right\} \oplus \mathrm{U}^{4} \subseteq[(\mathrm{UV}) \mathrm{V}] \mathrm{V} \oplus \mathrm{U}^{4}$, pois $\mathrm{u}_{0} \in(\mathrm{UV}) \mathrm{V}$ e $\mathrm{U}^{4} \subseteq \mathrm{V}$. Logo, $\left[\left(\mathrm{U}_{0} \mathrm{~V}_{0}\right) \mathrm{V}_{0}\right] \mathrm{V}_{0} \oplus \mathrm{U}_{0}^{4} \subseteq[(\mathrm{UV}) \mathrm{V}] \mathrm{V} \oplus \mathrm{U}^{4}$, o que mostra que este último $\mathrm{P}$-subespaço é S-invariante.

Lema 7.21 Se $S \subseteq \operatorname{ann}_{\mathrm{U}}\{U(U V)\} \cup U V$, então $p(U, V)=U[U(U V)] \oplus$ $\oplus U[(U V) V]$ é S-invariante.

Prova: De fato, pela Proposição 9 temos $\mathrm{U}_{0}\left[\left(\mathrm{U}_{0} \mathrm{~V}_{0}\right) \mathrm{V}_{0}\right] \subseteq \mathrm{U}[\mathrm{U}(\mathrm{UV})] \oplus$ $\oplus \mathrm{U}[(\mathrm{UV}) \mathrm{V}]$. Por outro lado, segue da Proposição 8 que $\mathrm{U}_{0}\left[\mathrm{U}_{0}\left(\mathrm{U}_{0} \mathrm{~V}_{0}\right)\right] \subseteq$ $\subseteq \mathrm{U}[\mathrm{U}(\mathrm{UV})] \oplus \mathrm{u}_{0}\{\mathrm{U}[\mathrm{U}(\mathrm{UV})]\}$. Mostremos que, se $\mathrm{u}_{0} \in \operatorname{ann}_{\mathrm{U}}\{\mathrm{U}(\mathrm{UV})\} \cup$ $\cup \mathrm{UV}$, então $\mathrm{u}_{0}\{\mathrm{U}[\mathrm{U}(\mathrm{UV})]\} \subseteq \mathrm{U}[(\mathrm{UV}) \mathrm{V}]$. Para isso, consideremos $\mathrm{u}_{0} \in$ $\in a n_{U}\{U(U V)\}$ e $u_{1}\left[u_{2}(u v)\right] u m$ gerador de $U[U(U V)]$. Da identidade (4.4) aplicada a $\mathrm{u}_{0}, \mathrm{u}_{1} \in \mathrm{U}$ e $\mathrm{u}_{2}(\mathrm{uv}) \in \mathrm{V}$, temos $\mathrm{u}_{0}\left\{\mathrm{u}_{1}\left[\mathrm{u}_{2}(\mathrm{uv})\right]\right\}=$ $=-\mathrm{u}_{1}\left\{\mathrm{u}_{0}\left[\mathrm{u}_{2}(\mathrm{uv})\right]\right\}=0$, pois $\left.\mathrm{u}_{0}\left[\mathrm{u}_{2}(\mathrm{uv})\right]\right\}=0$ e assim, $0=\mathrm{u}_{0}\left\{\mathrm{u}_{1}\left[\mathrm{u}_{2}(\mathrm{uv})\right]\right\} \in$ $\in \mathrm{U}[(\mathrm{UV}) \mathrm{V}]$. Finalmente, tomando $\mathrm{u}_{0} \in \mathrm{UV}$, segue que $\mathrm{u}_{0}\{\mathrm{U}[\mathrm{U}(\mathrm{UV})]\} \subseteq$ $\subseteq(\mathrm{UV})(\mathrm{UV})=(\mathrm{UV})^{2}=\mathrm{U}[(\mathrm{UV}) \mathrm{V}]$. Portanto, se $\mathrm{S} \subseteq \operatorname{ann}_{\mathrm{U}}\{\mathrm{U}(\mathrm{UV})\} \cup$ $\mathrm{U} \mathrm{UV}$, então $\mathrm{U}_{0}\left[\mathrm{U}_{0}\left(\mathrm{U}_{0} \mathrm{~V}_{0}\right)\right] \oplus \mathrm{U}_{0}\left[\left(\mathrm{U}_{0} \mathrm{~V}_{0}\right) \mathrm{V}_{0}\right] \subseteq \mathrm{U}[\mathrm{U}(\mathrm{UV})] \oplus \mathrm{U}[(\mathrm{UV}) \mathrm{V}]$, o que mostra que este último $\mathrm{P}$-subespaço é $\mathrm{S}$-invariante.

Lema 7.22 Se $p(U, V)=U[U(U V)]+[(U V) V] V$, então $S_{P}=$ $=a n n_{U}\{U[U(U V)]\} \cap \operatorname{ann}_{U}\{[(U V) V] V\}$.

Prova: De fato, das Proposições 8 e 10, respectivamente, obtemos as inclusões $\mathrm{U}_{0}\left[\mathrm{U}_{0}\left(\mathrm{U}_{0} \mathrm{~V}_{0}\right)\right] \subseteq \mathrm{U}[\mathrm{U}(\mathrm{UV})] \ominus \mathrm{u}_{0}\{\mathrm{U}[\mathrm{U}(\mathrm{UV})]\}$ e $\left[\left(\mathrm{U}_{0} \mathrm{~V}_{0}\right) \mathrm{V}_{0}\right] \mathrm{V}_{0} \subseteq$ $\subseteq[(\mathrm{UV}) \mathrm{V}] \mathrm{V} \oplus \mathrm{u}_{0}\{[(\mathrm{UV}) \mathrm{V}] \mathrm{V}\}$. Dessa forma, $\mathrm{u}_{0} \in \mathrm{S}_{\mathrm{P}}$ se e somente se $\mathrm{p}\left(\mathrm{U}_{0}, \mathrm{~V}_{0}\right) \subseteq \mathrm{p}(\mathrm{U}, \mathrm{V})$; isto é, se e somente se $\mathrm{u}_{0}\{\mathrm{U}[\mathrm{U}(\mathrm{UV})]+[(\mathrm{UV}) \mathrm{V}] \mathrm{V}\} \subseteq$ $\subseteq\{\mathrm{U}[\mathrm{U}(\mathrm{UV})]+[(\mathrm{UV}) \mathrm{V}] \mathrm{V}\} \cap \mathrm{V}$, ou seja, se e somente se $\mathrm{u}_{0}\{\mathrm{U}[\mathrm{U}(\mathrm{UV})]+$ 
$+[(\mathrm{UV}) \mathrm{V}] \mathrm{V}\}=0$, o que ocorre se e somente se $\mathrm{u}_{0} \in \operatorname{ann}_{\mathrm{U}}\{\mathrm{U}[\mathrm{U}(\mathrm{UV})]+$ $+[(\mathrm{UV}) \mathrm{V}] \mathrm{V}\}=\operatorname{ann}_{\mathrm{L}}\{\mathrm{U}[\mathrm{U}(\mathrm{UV})]\} \cap \operatorname{ann}_{\mathrm{L}}\{[(\mathrm{UV}) \mathrm{V}] \mathrm{V}\}$.

Lema 7.23 Se $p(U, V)=U^{3} V+[(U V) V] V$, então $S_{P}=a n n_{U}\left\{U^{3} V\right\} \cap$ $\cap \operatorname{ann}_{U}\{[(U V) V] V\}$.

Prova: Das Proposições 7 e 10, respectivamente, obtemos as inclusões $\mathrm{U}_{0}^{3} \mathrm{~V}_{0} \subseteq \mathrm{U}^{3} \mathrm{~V} \oplus \mathrm{u}_{0}\left(\mathrm{U}^{3} \mathrm{~V}\right)$ e $\left[\left(\mathrm{U}_{0} \mathrm{~V}_{0}\right) \mathrm{V}_{0}\right] \mathrm{V}_{0} \subseteq[(\mathrm{UV}) \mathrm{V}] \mathrm{V} \oplus \mathrm{u}_{0}\{[(\mathrm{UV}) \mathrm{V}] \mathrm{V}\}$ Portanto, $\mathrm{u}_{0} \in \mathrm{S}_{\mathrm{P}}$ se e somente se $\mathrm{p}\left(\mathrm{U}_{0}, \mathrm{~V}_{0}\right) \subseteq \mathrm{p}(\mathrm{U}, \mathrm{V})$ e isto ocorre se e somente se $\mathrm{u}_{0}\left\{\mathrm{U}^{3} \mathrm{~V}+[(\mathrm{UV}) \mathrm{V}] \mathrm{V}\right\} \subseteq\left\{\mathrm{U}^{3} \mathrm{~V}+[(\mathrm{UV}) \mathrm{V}] \mathrm{V}\right\} \cap \mathrm{V}$; ou seja, se e somente se $\mathrm{u}_{0}\left\{\mathrm{U}^{3} \mathrm{~V}+[(\mathrm{UV}) \mathrm{V}] \mathrm{V}\right\}=0$, o que ocorre se e somente se $\mathrm{u}_{0} \in$ $\left.\in \operatorname{ann}_{\mathrm{U}}\left\{\mathrm{U}^{3} \mathrm{~V}+[(\mathrm{UV}) \mathrm{V}] \mathrm{V}\right\}=\operatorname{ann}_{\mathrm{U}}\left\{\mathrm{U}^{3} \mathrm{~V}\right)\right\} \cap \operatorname{ann}_{\mathrm{U}}\{[(\mathrm{UV}) \mathrm{V}] \mathrm{V}\}$.

Lema 7.24 Se $S \subseteq \operatorname{ann}_{\mathrm{U}}\left\{U^{4}\right\} \cap \operatorname{ann}_{\mathrm{U}}\{U[(U V) V]\}$, então $p(U, V)=$ $=U^{+}+U[(U V) V]$ é S-invariante.

Prova: Das Proposições 6 e 9, respectivamente, obtemos as inclusões $\mathrm{U}_{0}^{4} \subseteq \mathrm{u}_{0} \mathrm{U}^{4} \oplus \mathrm{U}^{4}$ e $\mathrm{U}_{0}\left[\left(\mathrm{U}_{0} \mathrm{~V}_{0}\right) \mathrm{V}_{0}\right] \subseteq \mathrm{u}_{0} \mathrm{U}[(\mathrm{UV}) \mathrm{V}] \oplus \mathrm{U}[(\mathrm{UV}) \mathrm{V}]$. Logo, se $\mathrm{u}_{0} \in \operatorname{ann}_{\mathrm{U}}\left\{\mathrm{U}^{4}\right\} \cap \operatorname{ann}_{\mathrm{U}}\{\mathrm{U}[(\mathrm{UV}) \mathrm{V}]\}$, segue que $\mathrm{U}_{0}^{4}+\mathrm{U}_{0}\left[\left(\mathrm{U}_{0} \mathrm{~V}_{0}\right) \mathrm{V}_{0}\right] \subseteq$ $\subseteq \mathrm{U}^{4}+\mathrm{U}[(\mathrm{UV}) \mathrm{V}]$, e portanto este último P-subespaço é S-invariante. 


\section{Apêndice}

Neste apêndice, colocamos a lista completa dos 89 subespaços polinomiais que obtivemos considerando os $\mathrm{P}$-subespaços obtidos a partir dos P-monômios de graus menores ou iguais a 4. São eles:

1. U

2. $\mathrm{V}$

3. $\mathrm{U}^{2}$

4. UV

5. $\mathrm{U}^{3}$

6. $\mathrm{U}(\mathrm{UV})$

7. (UV)V

8. $\mathrm{U}^{4}$

9. $\mathrm{U}[\mathrm{U}(\mathrm{UV})]$

10. $\mathrm{U}[(\mathrm{UV}) \mathrm{V}]$

11. $\mathrm{U}^{3} \mathrm{~V}$

12. $[(\mathrm{UV}) \mathrm{V}] \mathrm{V}$

13. $\mathrm{U} \oplus \mathrm{U}^{2}$

14. $\mathrm{U} \oplus \mathrm{U}(\mathrm{UV})$

15. $\mathrm{U} \oplus \mathrm{U}^{4}$

16. $\mathrm{U} \oplus \mathrm{U}[(\mathrm{UV}) \mathrm{V}]$

17. $\mathrm{UV} \oplus \mathrm{V}$

18. $\mathrm{U}^{3} \oplus \mathrm{V}$

19. $(\mathrm{UV}) \mathrm{V} \oplus \mathrm{V}$ 
20. $\mathrm{U}[\mathrm{U}(\mathrm{UV})] \oplus \mathrm{V}$

21. $\mathrm{U}^{3} \mathrm{~V} \oplus \mathrm{V}$

22. $[(\mathrm{UV}) \mathrm{V}] \mathrm{V} \oplus \mathrm{V}$

23. $\mathrm{UV} \oplus \mathrm{U}^{2}=\mathrm{N}^{2}$

24. $\mathrm{U}^{3} \oplus \mathrm{U}^{2}$

25. $(\mathrm{UV}) \mathrm{V} \oplus \mathrm{U}^{2}$

26. $\mathrm{U}[\mathrm{U}(\mathrm{UV})] \oplus \mathrm{U}^{2}$

27. $\mathrm{U}^{3} \mathrm{~V} \oplus \mathrm{U}^{2}$

28. $[(\mathrm{UV}) \mathrm{V}] \mathrm{V} \oplus \mathrm{U}^{2}$

29. $\quad \mathrm{UV} \oplus \mathrm{U}(\mathrm{UV})$

30. $\mathrm{UV} \oplus \mathrm{U}^{4}$

31. $\mathrm{UV} \oplus \mathrm{U}[(\mathrm{UV}) \mathrm{V}]$

32. $\mathrm{U}^{3} \oplus \mathrm{U}(\mathrm{UV})$

33. $\mathrm{U}^{3}+(\mathrm{UV}) \mathrm{V}$

34. $U^{3} \oplus \mathrm{U}^{4}$

35. $\mathrm{U}^{3} \oplus \mathrm{U}[(\mathrm{UV}) \mathrm{V}]$

36. $\mathrm{U}^{3}+[(\mathrm{UV}) \mathrm{V}] \mathrm{V}$

37. $(\mathrm{UV}) \mathrm{V} \oplus \mathrm{U}(\mathrm{UV})$

38. $\mathrm{U}[\mathrm{U}(\mathrm{UV})] \oplus \mathrm{U}(\mathrm{UV})$

39. $U^{3} \mathrm{~V} \oplus \mathrm{U}(\mathrm{UV})$

40. $[(\mathrm{UV}) \mathrm{V}] \mathrm{V} \oplus \mathrm{U}(\mathrm{UV})$

41. $(\mathrm{UV}) \mathrm{V} \oplus \mathrm{U}^{4}$

42. (UV) $\mathrm{V}+\mathrm{U}[\mathrm{U}(\mathrm{UV})]$

43. $(\mathrm{UV}) \mathrm{V} \oplus \mathrm{U}[(\mathrm{UV}) \mathrm{V}]$

44. $\mathrm{U}[\mathrm{U}(\mathrm{UV})] \oplus \mathrm{U}^{4}$

45. $\mathrm{U}^{4}+\mathrm{U}[(\mathrm{UV}) \mathrm{V}]$

46. $\mathrm{U}^{3} \mathrm{~V} \oplus \mathrm{U}^{4}$

47. $[(\mathrm{UV}) \mathrm{V}] \mathrm{V} \oplus \mathrm{U}^{4}$

48. $\mathrm{U}[\mathrm{U}(\mathrm{UV})] \oplus \mathrm{U}[(\mathrm{UV}) \mathrm{V}]$

49. $\mathrm{U}[\mathrm{U}(\mathrm{UV})]+[(\mathrm{UV}) \mathrm{V}] \mathrm{V}$

50. $\mathrm{U}^{3} \mathrm{~V} \oplus \mathrm{U}[(\mathrm{UV}) \mathrm{V}]$

51. $[(\mathrm{UV}) \mathrm{V}] \mathrm{V} \oplus \mathrm{U}[(\mathrm{UV}) \mathrm{V}]$

52. $\mathrm{U}^{3} \mathrm{~V}+[(\mathrm{UV}) \mathrm{V}] \mathrm{V}$ 
53. $\quad \mathrm{U} \oplus\left\{\mathrm{U}^{4}+\mathrm{U}[(\mathrm{UV}) \mathrm{V}]\right\}$

54. $\left[\mathrm{U}^{3}+(\mathrm{UV}) \mathrm{V}\right] \ominus \mathrm{V}$

55. $\left\{\mathrm{U}^{3}+[(\mathrm{UV}) \mathrm{V}] \mathrm{V}\right\} \oplus \mathrm{V}$

56. $\{(\mathrm{UV}) \mathrm{V}+\mathrm{U}[\mathrm{U}(\mathrm{UV})]\} \oplus \mathrm{V}$

57. $\{\mathrm{U}[\mathrm{U}(\mathrm{UV})]+[(\mathrm{UV}) \mathrm{V}] \mathrm{V}\} \oplus \mathrm{V}$

58. $\left\{\mathrm{U}^{3} \mathrm{~V}+[(\mathrm{UV}) \mathrm{V}] \mathrm{V}\right\} \oplus \mathrm{V}$

59. $\left[\mathrm{U}^{3}+(\mathrm{UV}) \mathrm{V}\right] \oplus \mathrm{U}^{2}$

60. $\left\{\mathrm{U}^{3}+[(\mathrm{UV}) \mathrm{V}] \mathrm{V}\right\} \oplus \mathrm{U}^{2}$

61. $\{(\mathrm{UV}) \mathrm{V}+\mathrm{U}[\mathrm{U}(\mathrm{UV})]\} \oplus \mathrm{U}^{2}$

62. $\{\mathrm{U}[\mathrm{U}(\mathrm{UV})]+[(\mathrm{UV}) \mathrm{V}] \mathrm{V}\} \oplus \mathrm{U}^{2}$

63. $\left\{\mathrm{U}^{3} \mathrm{~V}+[(\mathrm{UV}) \mathrm{V}] \mathrm{V}\right\} \oplus \mathrm{U}^{2}$

64. $\mathrm{UV} \oplus\left\{\mathrm{U}^{4}+\mathrm{U}[(\mathrm{UV}) \mathrm{V}]\right\}$

65. $\left[\mathrm{U}^{3}+(\mathrm{UV}) \mathrm{V}\right] \oplus \mathrm{U}(\mathrm{UV})=\mathrm{N}^{3}$

66. $\left\{\mathrm{U}^{3}+[(\mathrm{UV}) \mathrm{V}] \mathrm{V}\right\} \oplus \mathrm{U}(\mathrm{UV})$

67. $\left[\mathrm{U}^{3}+(\mathrm{UV}) \mathrm{V}\right] \oplus \mathrm{U}^{4}$

68. $\left[\mathrm{U}^{3}+(\mathrm{UV}) \mathrm{V}\right] \oplus \mathrm{U}[(\mathrm{UV}) \mathrm{V}]$

69. $\mathrm{U}^{3} \oplus\left\{\mathrm{U}^{4}+\mathrm{U}[(\mathrm{UV}) \mathrm{V}]\right\}$

70. $\left\{\mathrm{U}^{3}+[(\mathrm{UV}) \mathrm{V}] \mathrm{V}\right\} \oplus \mathrm{U}^{4}$

71. $\left\{\mathrm{U}^{3}+[(\mathrm{UV}) \mathrm{V}] \mathrm{V}\right\} \oplus \mathrm{U}[(\mathrm{UV}) \mathrm{V}]$

72. $\{(\mathrm{UV}) \mathrm{V}+\mathrm{U}[\mathrm{U}(\mathrm{UV})]\} \oplus \mathrm{U}(\mathrm{UV})$

73. $\{\mathrm{U}[\mathrm{U}(\mathrm{UV})]+[(\mathrm{UV}) \mathrm{V}] \mathrm{V}\} \oplus \mathrm{U}(\mathrm{UV})$

74. $\left\{\mathrm{U}^{3} \mathrm{~V}+[(\mathrm{UV}) \mathrm{V}] \mathrm{V}\right\} \oplus \mathrm{U}(\mathrm{UV})$

75. $\{(\mathrm{UV}) \mathrm{V}+\mathrm{U}[\mathrm{U}(\mathrm{UV})]\} \oplus \mathrm{U}^{4}$

76. $(\mathrm{UV}) \mathrm{V} \oplus\left\{\mathrm{U}^{4}+\mathrm{U}[(\mathrm{UV}) \mathrm{V}]\right\}$

77. $\{(\mathrm{UV}) \mathrm{V}+\mathrm{U}[\mathrm{U}(\mathrm{UV})]\} \oplus \mathrm{U}[(\mathrm{UV}) \mathrm{V}]$

78. $\mathrm{U}[\mathrm{U}(\mathrm{UV})] \oplus\left\{\mathrm{U}^{4}+\mathrm{U}[(\mathrm{UV}) \mathrm{V}]\right\}$

79. $\{\mathrm{U}[\mathrm{U}(\mathrm{UV})]+[(\mathrm{UV}) \mathrm{V}] \mathrm{V}\} \oplus \mathrm{U}^{4}$

80. $\mathrm{U}^{3} \mathrm{~V} \oplus\left\{\mathrm{U}^{4}+\mathrm{U}[(\mathrm{UV}) \mathrm{V}]\right\}$

81. $[(\mathrm{UV}) \mathrm{V}] \mathrm{V} \oplus\left\{\mathrm{U}^{4}+\mathrm{U}[(\mathrm{UV}) \mathrm{V}]\right\}$

82. $\left\{\mathrm{U}^{3} \mathrm{~V}+[(\mathrm{UV}) \mathrm{V}] \mathrm{V}\right\} \oplus \mathrm{U}^{4}$

83. $\{\mathrm{U}[\mathrm{U}(\mathrm{UV})]+[(\mathrm{UV}) \mathrm{V}] \mathrm{V}\} \oplus \mathrm{U}[(\mathrm{UV}) \mathrm{V}]$

84. $\left\{\mathrm{U}^{3} \mathrm{~V}+[(\mathrm{UV}) \mathrm{V}] \mathrm{V}\right\} \oplus \mathrm{U}[(\mathrm{UV}) \mathrm{V}]$

85. $\left[\mathrm{U}^{3}+(\mathrm{UV}) \mathrm{V}\right] \oplus\left\{\mathrm{U}^{4}+\mathrm{U}[(\mathrm{UV}) \mathrm{V}]\right\}$ 
86. $\left\{\mathrm{U}^{3}+[(\mathrm{UV}) \mathrm{V}] \mathrm{V}\right\} \oplus\left\{\mathrm{U}^{4}+\mathrm{U}[(\mathrm{UV}) \mathrm{V}]\right\}$

87. $\left\{(\mathrm{UV}) \mathrm{V}+\mathrm{U}[\mathrm{U}(\mathrm{UV})] \oplus\left\{\mathrm{U}^{+}+\mathrm{U}[(\mathrm{UV}) \mathrm{V}]\right\}\right.$

88. $\{\mathrm{U}[\mathrm{U}(\mathrm{UV})]+[(\mathrm{UV}) \mathrm{V}] \mathrm{V}\} \oplus\left\{\mathrm{U}^{4}+\mathrm{U}[(\mathrm{UV}) \mathrm{V}]\right\}=\mathrm{N}^{4}$

89. $\left\{\mathrm{U}^{3} \mathrm{~V}+[(\mathrm{UV}) \mathrm{V}] \mathrm{V}\right\} \oplus\left\{\mathrm{U}^{4}+\mathrm{U}[(\mathrm{UV}) \mathrm{V}]\right\}$ 


\section{Bibliografia}

[1] ABRAhAM, V. M., A note on train algebras, Proceedings of the Edinburgh Mathematical Society (2) 20 53-58 (1976).

[2] BENAVIDES, R., Associative bilinear forms in T-algebras of rank 3. (preprint).

[3] BENAVIDES, R., Sobre as t-álgebras de posto 3 e questões correlatas, Tese de Doutorado, IME-USP, 1995.

[4] BENAVIDES, R., COSTA, R. and MALLOL, C., Weak isotopy in train algebras, Nova Journal of Mathematics, Game Theory, and Algebra (to appear)

[5] BENAVIDES, R., MALLOL, C., Peirce transformations in train algebras of rank 3. (preprint).

[6] COSTA, R., Principal train algebras of rank 3 and dimension $\leq$ 5, Proceedings of the Edinburgh Mathematical Society 33 61-70 (1990).

[7] COSTA, R., On train algebras of rank 3, Linear Algebra and its Applications 148 1-12 (1991).

[8] COSTA, R., GUZZO, Jr H. and VICENTE, P., Shape identities in train algebras of rank 3. (submetido).

[9] COSTA, R., SUAZO, A., The multiplication algebra of a train algebra of rank 3, Nova Journal of Mathematics, Game Theory, and Algebra, vol. 5, n.3, 287-298 (1996). 
[10] Díaz, M. C. L., Superálgebras de Bernstein y sus supermódulos, Tese de Doutorado, Departamento de Matemáticas. Universidad de Oviedo, 1997.

[11] ETHERINGTON, I. M. H., On non-associative combinations, Proceedings Royal Society Edinburgh. 59 153-162 (1939).

[12] ETHERINGTON, I. M. H., Genetic algebras, Proceedings Royal Society Edinburgh 59 242-258 (1939).

[13] ETHERINGTON, I. M. H., Special train algebras, Quarterly Journal of Mathematics (Oxford), vol 12 1-8 (1941).

[14] ETHERINGTON, I. M. H., Commutative train algebras of ranks 2 and 3, Journal of London Mathematical Society 15 136-149 (1940) Corrigendum ibid 20238 (1945).

[15] ETHERINGTON, I. M. H., Non-commutative train algebras of ranks 2 and 3, Proceedings of the London Mathematical Society (2) 52 241-252 (1951).

[16] GUZZO, Jr H., The Peirce decomposition for commutaive train algebras, Communications in Algebra 22 (14) 5745-5757 (1994).

[17] GUZZO, Jr H., The bar radical of baric algebras, Archives of Mathematics vol. 67 106-118 (1996).

[18] GUZZO, Jr H., Embedding nil algebras in train algebras, Proceedings of the Edinburgh Mathematical Society 37 463-470 (1994).

[19] GUZZO, Jr H., On normal and composition series for baric algebras, Nova Journal of Mathematics, Game Theory, and Algebra 4(1) 25-38 (1995).

[20] GUZZO, Jr H., VICENTE P., Some properties of commutative train algebras of rank 3, Communications in Algebra (to appear). 
[21] GUZZO, Jr H., VICENTE, P., Train algebras of rank $n$ which are Bernstein or power-associative algebras. Nova Journal of Mathematics, Game Theory, and Algebra (to appear).

[22] HOLGATE, Ph., Free non-associative principal train algebras, Proceedings of the Edinburgh Mathematical Society 27 313-319 (1984).

[23] HOLGATE, Ph., Some train algebras that are special triangular, The Mathematics Student 55 (2-4) 135-139 (1987).

[24] HOLGATE, Ph., A train algebra that is not special triangular, Archives of Mathematics 50 122-124 (1988).

[25] LYUBICH, Yu. I., Mathematical Structures in Population Genetics, Biomathematics 22, Springer, Berlin-Heidelberg-New York (1992).

[26] OUATTARA, M., Sur les T-algèbres de Jordan, Linear Algebra and its Applications 144 11-21 (1991).

[27] OUATTARA, M., Sur les algèbres de Bernstein qui sont des Talgèbres, Linear Algebra and its Applications 148 171-178 (1991).

[28] REED, M. L., Algebraic Structure of Genetic Inheritance, Bulletin (New Series) of the American Mathematical Society vol. 34, n. 2, April 1997, 107-130

[29] SCHAFER, R. D., Structure of genetic algebras, American Journal of Mathematics 71 121-135 (1949).

[30] SCHAFER, R. D., Introduction to nonassociative algebras, Academic Press, New York, (1966).

[31] SUAZO, A., Train algebras de rango 3. (preprint).

[32] WALCHER, S., Algebras which satisfy a train equation for the first three plenary powers, Archives of Mathematics 56 547-551 (1991).

[33] WALCHER, S., On Bernstein algebras which are train algebras, Proceedings of the Edinburgh Mathematical Society 35 159-166 (1992). 
[34] WALCHER, S., Algebras of rank three. S. Gonzalez (ed.): Nonassociative algebra and its applications. Mathematics and its Applications 303, Kluwer, Dordrechet (1994).

[35] WALKhOFF, A., Algèbres de Composition Faible, Thèse de Doctorat, Université de Montpellier, 1996.

[36] WÖRZ, A., Algebras in Genetics, Lecture Notes in Biomathematics 36, Berlin-Heidelberg-New York (1980).

[37] ZHEVlAKOV, K. A., SLINKO A. M., SHESTAKOV I. P., SHIRSKOV A. I., Rings that are nearly associative, Pure and Applied Mathematics, Academic Press (1982). 